\title{
Physiology, metabolism and redox mechanisms in chronic cardiac volume overload
}

\author{
Doctoral Thesis \\ In partial fulfillment of the requirements for the degree \\ "Doctor of Philosophy (Ph.D.)" \\ in the Molecular Medicine Study Programme \\ at the Georg-August University Göttingen
}

submitted by

Moritz Thomas Schnelle

born in Bochum

Göttingen 2016 


\section{Members of the Thesis Committee:}

Supervisor:

Prof. Dr. med. Dörthe M. Katschinski

Department of Cardiovascular Physiology; University Medical Center, Georg-August University Göttingen

Humboldtallee 23, 37073 Göttingen, Germany

Co-Supervisors:

Prof. Ajay M. Shah

The James Black Centre, King's College London

125 Coldharbour Lane, London SE5 9NU, UK

Prof. Dr. med. Gerd Hasenfuß

Department of Cardiology and Pneumology; University Medical Center, Georg-August University Göttingen

Robert-Koch-Str. 40, 37075 Göttingen, Germany

Second member of the Thesis Committee:

Prof. Dr. rer. nat. Blanche Schwappach

Department of Molecular Biology; University Medical Center, Georg-August University Göttingen

Humboldtallee 23, 37073 Göttingen, Germany

Third member of the Thesis Committee:

Prof. Dr. rer. nat. Viacheslav O. Nikolaev

Institute of Experimental Cardiovascular Research; University Medical Center HamburgEppendorf

Martinistr. 52, 20246 Hamburg, Germany

$\begin{array}{lllll}\text { Date of } & \text { disputation: } & \text { September } & 2016\end{array}$




\section{Affidavit}

Here I declare that my doctoral thesis entitled "Physiology, metabolism and redox mechanisms in chronic cardiac volume overload" has been written independently with no other sources and aids than quoted.

Moritz Thomas Schnelle

Göttingen, July 2016 


\section{Table of Contents}

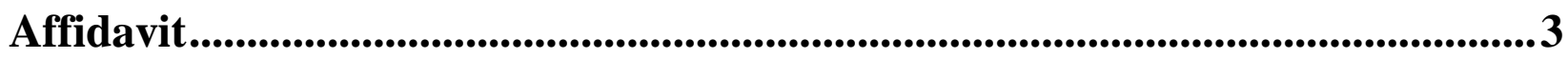

Table of Contents.................................................................................................................................4

Acknowledgements......................................................................................................................7

Statement of Conjoint Work ...........................................................................................................8 8

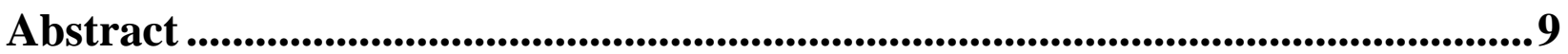

Index of Figures .............................................................................................................. 11

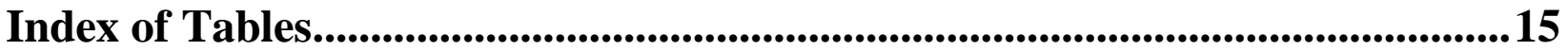

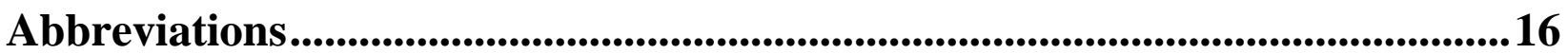

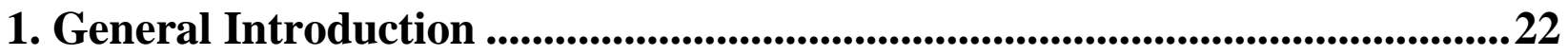

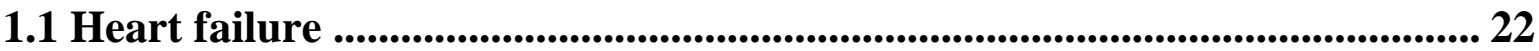

1.2 Cardiac remodelling................................................................................................... 23

1.3 Pressure versus volume haemodynamic load ......................................................... 25

1.4 Cardiac metabolism in heart failure ............................................................................ 27

1.5 Reactive oxygen species (ROS) in heart failure..................................................... 30

1.6 NADPH oxidases in the heart ....................................................................... 32

1.7 Aims .......................................................................................................................................... 36

2. Material and Methods.......................................................................................................37

2.1 Gene-modified mice .......................................................................................................... 37

2.2 Genotyping .................................................................................................................................. 37

2.3 Murine volume overload model ....................................................................................... 39

2.4 Murine pressure overload models ..................................................................40

2.5 Infusion of ${ }^{13} \mathrm{C}$-labelled glucose in mice, sample preparation and ${ }^{13} \mathrm{C}-\mathrm{NMR}$ -

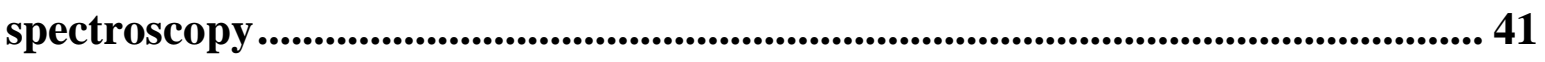

2.6 Organ harvesting.................................................................................................................. 42

2.7 Assessment of kidney function in mice....................................................................... 43

2.8 Echocardiography ............................................................................................................. 44 
2.9 RNA isolation, cDNA synthesis and quantitative real-time polymerase chain reaction (qRT-PCR).......................................................................................................... 45

2.10 Protein isolation and Western Blotting ................................................................. 48

2.11 Histology...........................................................................................................................5 50

2.11.1 Apoptosis__ 50

2.11.2 Fibrosis _ 51

2.11.3 Cardiomyocyte area and capillary density __ 51

2.12 Glutathione measurements.......................................................................................... 51

2.13 Statistical analysis ...................................................................................................... 52

3. Results I: Non-invasive assessment of cardiac physiology in murine models of volume and pressure overload ...................................................................53

3.1 Introduction ...................................................................................................................... 53

3.2 Basic characterization of the aortocaval fistula (Shunt), a model to induce volume overload in mice ................................................................................................... 55

3.2.1 Transthoracic echocardiography __ 55

3.2.2 Heart and organ weights _ـ 58

3.2.3 Kidney function __ 61

3.2.4 Gene expression _ 61

3.3 Non-invasive assessment of cardiac function in mice during pressure and volume overload in mice using state-of-the-art echocardiography ...................... 63

3.3.1 Systolic function _ 63

3.3.2 Diastolic function _ 64

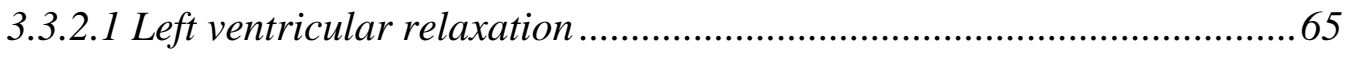

3.3.2.2 Left ventricular filling ......................................................................66

3.3.2.3 Diastolic strain...................................................................................68

3.3.3 Systolic and diastolic function following transverse aortic constriction__ 69

3.3.4 Correlation of diastolic parameters with cardiac hypertrophy following pressure overload — 70

3.4 Discussion...................................................................................................................... 72

4. Results II: Cardiac metabolism in murine models of volume and pressure overload ........................................................................................................................................... 77

4.1 Introduction .......................................................................................................................... 77 
4.2. Basic characterization of cardiac responses to two weeks of pressure and volume overload in mice ..................................................................................... 79

4.2.1 Cardiac remodelling, function and hypertrophy __ 79

4.3 Changes in cardiac glucose metabolism in mice two weeks after pressure and volume overload .......................................................................................................... 84

4.3.1 Glycolysis _ 84

4.3.2 TCA cycle

4.3.3 Glutamine metabolism _ 95

4.3.4 Gene expression of enzymes related to fatty acid oxidation (FAO) and fatty acid synthesis (FAS)

4.4 Discussion............................................................................................................................. 102

\section{Results III: The role of Nox4 in cardiac remodelling during chronic volume}

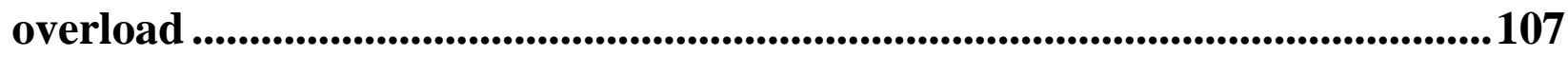

5.1 Introduction . 107

5.2. Assessment of Nox4-involvement in cardiac eccentric remodelling following two weeks of volume overload in mice ........................................................................ 109

5.2.1 Expression profiles of NADPH oxidases and related genes in the heart following chronic volume overload

5.2.2 Cardiac remodelling and hypertrophy in global Nox4-null mice and WT littermates following two weeks of volume overload

5.2.3 Cardiac stress, angiogenesis, fibrosis and apoptosis in global Nox4-null mice and WT littermates following two weeks of volume overload

5.2.4 Cardiac kinase and protein synthesis activation in global Nox4-null mice and WT littermates following two weeks of volume overload

5.2.5 Cardiac remodelling and hypertrophy in cardiomyocyte-specific Nox4 overexpressing mice (Nox4tg) and WT littermates following two weeks of volume overload

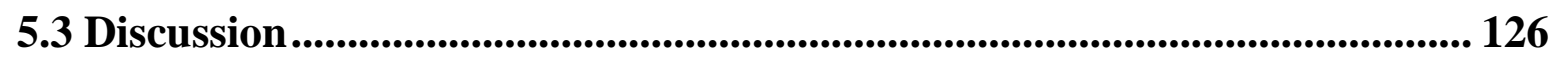

6. Concluding Discussion .......................................................................................................131

References ................................................................................................................................... 137

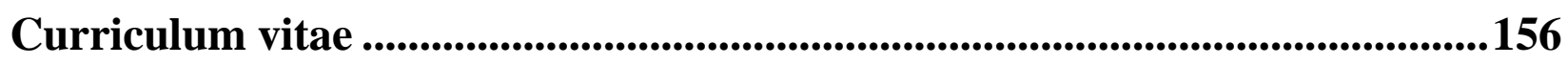




\section{Acknowledgements}

Firstly, I want to thank my two supervisors, Prof. Ajay Shah and Prof. Gerd Hasenfuß, for their great support throughout the last three years. They allowed me to generate and pursue own ideas, which was crucial to engage with my project and to get the most out of my $\mathrm{PhD}$. Their supervision created an ideal environment, both on a professional and personal level, in order for me to develop as a basic scientist. The intellectual exchange has enriched my understanding and appreciation of basic sciences, particularly of cardiac biology and physiology. This will have a significant impact on my career both as an academic and a clinician.

I also would like to thank all members of the Shah- and Hasenfuß-laboratories for their contributions and consistent support during various experiments. They all have a significant impact on the success and completion of this thesis. I am particularly grateful to Dr. Greta Sawyer and Dr. Helena Zhang for performing abdominal aortic banding and transverse aortic constriction surgeries, Norman Catibog for helping with murine echocardiography, Dr. Mei Chong for carrying out experiments involving NMR and Priv.-Doz. Dr. med. Karl Toischer for teaching me the aortocaval fistula (Shunt) surgery technique.

I also want to thank Prof. Dörthe Katschinski for being an outstanding mentor throughout the last eight years and for her continuous support during my early career. The interaction with her is and always has been invaluable with respect to my professional and personal development. Additionally, I want to thank her, Prof. Blanche Schwappach and Prof. Viacheslav Nikolaev for being part of my thesis committee and their stimulating, intellectual input. Moreover, I highly appreciate Prof. Susanne Lutz, Prof. Hubertus Jarry and Prof. Thomas Meyer for joining my thesis examination board.

I am grateful to the DFG for funding, which allowed me to undertake this work.

Finally, I am eternally thankful to my family, especially my parents. Their continuous love, encouragement, belief and trust in me paired with high levels of patience and understanding have been and always will be the best support to overcome all previous and future challenges in life. 


\section{Statement of Conjoint Work}

Chapter 3: Dr. Helena Zhang performed abdominal aortic banding (AAB), Dr. Greta Sawyer transverse aortic constriction (TAC) surgeries. Norman Catibog helped with the advanced echocardiographic techniques, especially measurements of diastolic parameters in mice.

Chapter 4: Dr. Greta Sawyer performed transverse aortic constriction (TAC) surgeries, Dr. Mei Chong carried out the experiments for metabolic flux analysis using NMR-spectroscopy. 


\section{Abstract}

Chronic haemodynamic stress in the heart, induced by volume or pressure overload, contributes to cardiac dysfunction and heart failure and is associated with several human cardiovascular diseases (e.g. valvular heart diseases, arterial hypertension). Volume and pressure overload induce distinct cardiac remodelling responses in humans and mouse models, including different signalling patterns, but the differences between them are incompletely understood. In order to comprehensively study these, murine models of volume overload (aortocaval fistula, Shunt) and pressure overload (transverse aortic constriction, TAC and abdominal aortic banding, AAB) were studied in this work. The overall aims were (a) to apply advanced non-invasive methods to characterize changes in diastolic physiology in the two models; (b) to compare the changes in glucose metabolism that occur during these haemodynamic stress conditions; (c) to study during chronic cardiac volume overload the role of NADPH oxidase-4 (Nox4), a reactive oxygen species (ROS)-generating enzyme recently found to be protective against chronic pressure overload.

Assessment of diastolic function in mice following chronic volume and pressure overload using state-of-the-art echocardiography revealed marked differences between the models with respect to left ventricular relaxation and filling. The isovolumic relaxation time (IVRT), left atrial area, $\mathrm{E} / \mathrm{E}^{\prime}$ and reverse longitudinal strain rate were found to be consistent and reproducible parameters to analyze diastolic properties in these haemodynamically different settings. For interpretation, however, the physiological and haemodynamic background needs to be well-understood.

A novel methodology of in vivo $\left[\mathrm{U}^{13} \mathrm{C}\right]$ glucose administration followed by isotopomer analysis using NMR-spectroscopy, as well as expression profiles of metabolic enzymes, revealed fundamental differences in cardiac glucose metabolism following chronic volume or pressure overload in mice. Despite very similar increases in left ventricular hypertrophy between TAC and Shunt, glycolysis, TCA cycle activity, glutamine synthesis and O-GlcNAcylation of proteins were significantly increased only following TAC. These findings together with a nearly unchanged glucose metabolism after Shunt suggest a much more pronounced metabolic complexity during pressure overload and concentric remodelling than during volume overload.

Nox4 was found to promote eccentric hypertrophy following two weeks of volume overload, as global Nox4-null mice $\left(\right.$ Nox $\left.^{4--}\right)$ developed significantly less left ventricular hypertrophy and dilation compared to WT littermates. This was attributed to a Nox4-dependent activation of Akt 
and its downstream targets S6 ribosomal protein and eIF4E-BP1, which are known to initiate protein synthesis. Despite its role in eccentric remodelling, Nox4 did not seem to alter cardiac function at this point.

This study provides novel data on cardiac physiology and metabolism using advanced echocardiographic techniques and a novel in vivo ${ }^{13} \mathrm{C}$-labelling methodology following volume versus pressure overload in mice. It also identifies a novel Nox4-regulated pathway, which appears to be important for cardiac adaptation during volume overload. These results might be of relevance for future heart failure therapy development. 


\section{Index of Figures}

Figure 1.1: Schematic illustration of concentric versus eccentric cardiac remodelling

Figure 1.2: Schematic illustration of NADPH oxidases with respect to their structure, regulation and cellular distribution (Adapted from Antioxid Redox Signal 2013; 18(9):1024-1041)

Figure 2.1: Schematic illustration of the aortocaval fistula (Shunt) surgery

Figure 2.2: Scheme of the transverse aortic constriction (TAC) surgery

Figure 2.3: Technical and physiological aspects of assessing the reverse longitudinal strain rate in murine echocardiography

Figure 3.1: Echocardiographic assessment of cardiac remodelling in mice following volume overload

Figure 3.2: Echocardiographic assessment of cardiac function in mice following volume overload

Figure 3.3: Cardiac hypertrophy following volume overload

Figure 3.4: Body and organ weights following volume overload

Figure 3.5: Kidney function following volume overload

Figure 3.6: Gene expression of cardiac stress markers in mice following volume overload

Figure 3.7: Assessment of heart rate and systolic function in murine models of pressure and volume overload using speckle tracking echocardiography

63

Figure 3.8: Sample placement for transmitral flow analysis

Figure 3.9: Assessment of the isovolumic relaxation time (IVRT) in murine models of pressure and volume overload using echocardiography

65 
Figure 3.10: Assessment of the E/E' ratio in murine models of pressure and volume overload using echocardiography

66

Figure 3.11: Assessment of the left atrial area in murine models of pressure and volume overload using echocardiography

Figure 3.12: Assessment of the reverse longitudinal strain rate in murine models of pressure and volume overload using speckle tracking echocardiography

Figure 3.13: Assessment of systolic and diastolic function in mice two weeks after transverse aortic constriction (TAC)

69

Figure 3.14: Correlation of diastolic parameters and cardiac hypertrophy in murine models of pressure overload

Figure 4.1: Echocardiographic assessment of cardiac remodelling in mice following two weeks of pressure and volume overload

Figure 4.2: Assessment of left ventricular function and hypertrophy in mice following two weeks of pressure or volume overload

Figure 4.3: Schematic illustration of the glycolytic pathway

Figure 4.4: Glycolytic activity in murine hearts following two weeks of pressure and volume overload

Figure 4.5: Cardiac gene expression of glycolytic enzymes in mice following two weeks of pressure and volume overload

\section{5}

Figure 4.6: Evaluation of different loading controls for Western Blotting in murine heart lysates following two weeks of pressure and volume overload

Figure 4.7: Cardiac protein expression of glycolytic enzymes in mice following two weeks of pressure and volume overload 
Figure 4.9: TCA cycle activity in murine hearts following two weeks of pressure and volume overload

Figure 4.10: Cardiac gene expression of enzymes related to TCA cycle metabolism in mice following two weeks of pressure and volume overload

Figure 4.11: Cardiac protein expression of enzymes related to TCA cycle metabolism in mice following two weeks of pressure and volume overload

Figure 4.12: Glutamine biosynthesis in murine hearts following two weeks of pressure and volume overload

\section{3}

Figure 4.13: Schematic illustration of glutamine-involvment in the hexosamine biosynthetic pathway (HBP) and glutathione synthesis

Figure 4.14: Cardiac protein expression of enzymes related to glutamine metabolism in mice following two weeks of pressure and volume overload

Figure 4.15: Glutamine-involvement in cardiac glutathione biosynthesis following two weeks of pressure and volume overload

Figure 4.16: Schematic illustration of fatty acid synthesis (FAS) and oxidation (FAO)

Figure 4.17: Cardiac gene expression of enzymes related to fatty acid oxidation (FAO) and synthesis (FAS) in mice following two weeks of pressure and volume overload

Figure 5.1: Cardiac gene expression of NADPH oxidases (Noxs) and subunits in WT mice following two weeks of volume overload

Figure 5.2: Cardiac protein expression and activation of NADPH oxidases (Noxs) in WT mice following two weeks of volume overload

Figure 5.3: Cardiac gene expression of Nrf2 (Nuclear factor erythroid-derived 2-like 2) targets in WT mice following two weeks of volume overload

Figure 5.4: Echocardiographic assessment of left ventricular remodelling and function in Nox $4^{-/-}$ mice and WT littermates following two weeks of volume overload 
Figure 5.5: Left ventricular hypertrophy in Nox $4^{-/-}$-mice and WT littermates following two weeks of volume overload

Figure 5.6: Cardiac gene expression of stress markers in Nox $4^{-/-}$-mice and WT littermates following two weeks of volume overload

\section{4}

Figure 5.7: Left ventricular capillary density in $\mathrm{Nox}^{4 /-}$-mice and WT littermates following two weeks of volume overload

115

Figure 5.8: Left ventricular interstitial fibrosis in Nox $4^{-/-}$-mice and WT littermates following two weeks of volume overload

116

Figure 5.9: Left ventricular apoptosis in Nox $4^{-/-}$-mice and WT littermates following two weeks of volume overload

117

Figure 5.10: Phosphorylation of cardiac Akt and Erk1/2 in Nox $4^{-/-}$-mice and WT littermates following two weeks of volume overload

Figure 5.11: Phosphorylation of cardiac S6 ribosomal protein and eIF4E-BP1 in Nox $4^{-/-}$-mice and WT littermates following two weeks of volume overload

120

Figure 5.12: Confirmation of Nox4 protein overexpression in heart lysates from cardiomyocytespecific Nox4 transgenic mice (Nox4tg)

121

Figure 5.13: Left ventricular remodelling, function and hypertrophy in Nox4tg-mice and WT littermates following two weeks of volume overload

122

Figure 5.14: Scheme of Nox4-involvement in the regulation of cardiac remodelling during pressure and volume overload

128 


\section{Index of Tables}

Table 2.1: Genotyping protocol for the global Nox4-null mouse line $\left(\operatorname{Nox} 4^{-/-}\right)$

Table 2.2: Genotyping protocol for the cardiomyocyte-specific Nox4 overexpressing mouse line (Nox4tg)

Table 2.3: Primer sequences for metabolic enzymes

Table 2.4: Primer sequences for genes involved in cardiac stress and redox signalling

Table 2.5: Antibodies detecting proteins involved in cardiac metabolism

Table 2.6: Antibodies detecting proteins involved in cardiac hypertrophy and redox signalling

Table 3.1: Echocardiographic parameters following volume overload

Table 4.1: Echocardiographic and morphometric data in mice following two weeks of pressure and volume overload

Table 5.1: Echocardiographic and morphometric data from Nox $4^{-/-}$-mice and WT littermates following two weeks of volume overload

Table 5.2: Echocardiographic and morphometric data from Nox4tg-mice and WT littermates following two weeks of volume overload

123 


\section{Abbreviations}

AAB

AAV

ACACA

ACACB

$\mathrm{ACCF}$

ACE

Acta1

ADP

AHA

Akt

Ang II

ANP

AS

ATP

Atp2a2

BNP

bpm

BSA

BW

CAMKII

cDNA

$\mathrm{C} / \mathrm{EBP} \beta$

cGMP

c-myc

$\mathrm{CO}_{2}$

CoA

CPT

CSA

Cyba

Cybb abdominal aortic banding

adeno-associated virus

acetyl-CoA carboxylase $\alpha$

acetyl-CoA carboxylase $\beta$

The American College of Cardiology Foundation

angiotensin converting enzyme

actin, alpha 1, skeletal muscle (gene encoding $\alpha$-skeletal actin)

adenosine diphosphate

The American Heart Association

protein kinase B

angiotensin II

atrial natriuretic peptide

aortic stenosis

adenosine triphosphate

ATPase, $\mathrm{Ca}^{2+}$ transporting, cardiac muscle, slow twitch 2 (gene encoding SERCA- $2 \alpha$

brain natriuretic peptide

beats per minute

bovine serum albumin

body weight

calcium/calmodulin-dependent protein kinase II

copy deoxyribonucleic acid

CCAAT-enhancer-binding protein $\beta$

cyclic guanosine monophosphate

cellular-myelocytomatosis (transcription factor)

carbon dioxide

coenzyme A

carnitine palmitoyltransferase

cross sectional area

cytochrome b-245, alpha polypeptide (gene encoding p22 ${ }^{\text {phox }}$ )

cytochrome b-245, beta polypeptide (gene encoding Nox2) 
DAPI

DCA

DNA

dNTP

DSS

DT

DUOX

E/A

EDTA

$\mathrm{E} / \mathrm{E}^{\prime}$

EF

e.g.

EGTA

eIF4E-BP1

E-loop

ERK1/2

ES

et al.

FAO

FAS

FASN

Fig.

FITC

FS

GAPDH

Gclc

GFAT

GLS 4',6-diamidino-2-phenylindole

dichloroacetate

deoxyribonucleic acid

deoxynucleotide triphosphates

4,4-dimethyl-4-silapentane-1-sulfonic acid

E-wave deceleration time

dual oxidase

ratio of early versus late diastolic transmitral flow velocity

ethylenediaminetetraacetic acid

ratio of early diastolic transmitral flow versus early diastolic mitral

annular velocity

ejection fraction

exempli gratia (engl.: for example)

ethylene glycol tetraacetic acid

eukaryotic translation initiation factor $4 \mathrm{E}$-binding protein 1

extracytosolic loop

extracellular signal-regulated kinases 1 and 2

embryonic stem

et alii (engl.: and others)

fatty acid oxidation

fatty acid synthesis

fatty acid synthase

figure

fluorescein isothiocyanate

fractional shortening

glyceraldehyde-3-phosphate dehydrogenase

glutamate-cysteine ligase, catalytic subunit

glutamine:fructose-6-phosphate aminotransferase

glutaminase 


\begin{tabular}{|c|c|}
\hline GLUT & glucose transporter \\
\hline GS & glutamine synthetase \\
\hline GSH & reduced glutathione \\
\hline GS-SG & oxidized glutathione \\
\hline Gst & glutathione S-transferase \\
\hline GTP & guanosine triphosphate \\
\hline $\mathrm{H}_{2} \mathrm{O}_{2}$ & hydrogen peroxide \\
\hline HBP & hexosamine biosynthetic pathway \\
\hline $\mathrm{HFpEF}$ & heart failure with preserved ejection fraction \\
\hline HFrEF & heart failure with reduced ejection fraction \\
\hline $\mathrm{HIF}-1 \alpha$ & hypoxia-inducible factor $1 \alpha$ \\
\hline HK & hexokinase \\
\hline HSQC & heteronuclear single quantum coherence \\
\hline $\mathrm{HW}$ & heart weight \\
\hline i.e. & id est (engl.: that is) \\
\hline IGF-1 & insulin-like growth factor-1 \\
\hline IVC & inferior vena cava \\
\hline IVRT & isovolumic relaxation time \\
\hline JNK & c-Jun NH2 terminal-kinase \\
\hline $\mathrm{KO}$ & knockout \\
\hline LA & left atrium \\
\hline $\mathrm{LDH}$ & lactate dehydrogenase \\
\hline LV & left ventricle \\
\hline LVEDD & left ventricular end-diastolic diameter \\
\hline LVESD & left ventricular end-systolic diameter \\
\hline MAO & monoamine oxidase \\
\hline MAPK & mitogen-activated protein kinase \\
\hline $\mathrm{MDH}$ & malate dehydrogenase \\
\hline ME & malic enzyme \\
\hline
\end{tabular}


MEF2

MI

MMP

mRNA

mTOR

$\mathrm{NAD}(\mathrm{P}) \mathrm{H}$

NCBI

Ncf11

Ncf2

Ncf4

NFAT

NMR

$\mathrm{NO}$

NOS

NOX

NOXA

NOXO

NP-40

Nppa

Nppb

Nrf2

n.s.

NYHA

$\mathrm{O}_{2}$

$\mathrm{O}_{2}^{-}$

O-GlcNAc

$\mathrm{OH}$

$\mathrm{ONOO}^{-}$

PBS myocyte enhance factor-2

myocardial infarction

matrix metalloproteinase

messenger ribonucleic acid

mammalian target of rapamycin

nicotinamide adenine dinucleotide (phosphate) hydrogen

National Center for Biotechnology Information

neutrophil cytosolic factor 1 (gene encoding $\mathrm{p} 47^{\mathrm{phox}}$ )

neutrophil cytosolic factor 2 (gene encoding p67 ${ }^{\text {phox }}$ )

neutrophil cytosolic factor 4 (gene encoding $\mathrm{p} 40^{\text {phox }}$ )

nuclear factor of activated T-cells

nuclear magnetic resonance

nitric oxide

nitric oxide synthase

NADPH oxidase

NADPH oxidase activator

NADPH oxidase organizer

nonyl phenoxypolyethanol

natriuretic peptide type A (gene encoding ANP)

natriuretic peptide type B (gene encoding BNP)

nuclear factor erythroid-derived 2-like 2

not significant

New York Heart Association (functional classification for the extent of heart failure

oxygen

superoxide

O-linked $\mathrm{N}$-acetylglucosamine

hydroxyl ion

peroxynitrite

phosphate-buffered saline 


\begin{tabular}{|c|c|}
\hline $\mathrm{PC}$ & pyruvate carboxylase \\
\hline $\mathrm{PCr}$ & phosphocreatine \\
\hline PCR & polymerase chain reaction \\
\hline $\mathrm{PDH}$ & pyruvate dehydrogenase \\
\hline PDK & pyruvate dehydrogenase kinase \\
\hline PET & positron emission tomography \\
\hline PFA & paraformaldehyde \\
\hline phox & phagocyte oxidase \\
\hline $\mathrm{PI} 3 \mathrm{~K}$ & phosphatidylinositol 3-kinase \\
\hline PKA & protein kinase $\mathrm{A}$ \\
\hline $\mathrm{PKC}$ & protein kinase $\mathrm{C}$ \\
\hline PKD & protein kinase D \\
\hline PKG & protein kinase $\mathrm{G}$ \\
\hline ppm & parts per million \\
\hline PPP & pentose phosphate pathway \\
\hline PTP & protein tyrosine phosphatase \\
\hline qRT-PCR & quantitative real-time polymerase chain reaction \\
\hline Rac & ras-related $\mathrm{C} 3$ botulinum toxin substrate \\
\hline RAS & renin angiotensin system \\
\hline RNA & ribonucleic acid \\
\hline RNS & reactive nitrogen species \\
\hline ROS & reactive oxygen species \\
\hline $\mathrm{rpm}$ & revolutions per minute \\
\hline $\mathrm{RR}$ & respiratory rate \\
\hline RV & right ventricle \\
\hline RyR2 & ryanodine receptor 2 \\
\hline SDS & sodium dodecyl sulfate \\
\hline SEM & standard error of the mean \\
\hline Ser & serine \\
\hline
\end{tabular}


SERCA- $2 \alpha$

SHUNT

Slc2a1

SNS

SR

TAC

TAE

TBST

TCA cycle

TCEP

TdT

Thr

Tris

TUNEL

Txnrd

Tyr

$\left[\mathrm{U}-{ }^{13} \mathrm{C}\right]$ glucose

UDP

UV

VEGF

VSMC

WGA

WT

$\mathrm{XO}$ sarcoplasmic/endoplasmic reticulum calcium ATPase-2 $\alpha$

murine aortocaval fistula model

solute carrier family 2 member 1 (gene encoding GLUT1)

sympathetic nervous system

sarcoplasmic reticulum

transverse aortic constriction

Tris-acetate-EDTA buffer

Tris-buffered saline and $0.01 \%$ Tween v/v

tricarboxylic acid cycle

Tris (2-carboxyethyl) phosphine hydrochloride

terminal deoxynucleotidyl transferase

threonine

Tris (hydroxymethyl) aminomethane

TdT-mediated dUTP-biotin nick end labelling

thioredoxin reductase

tyrosine

uniformly ${ }^{13} \mathrm{C}$-labelled glucose in all six carbons

uridine diphosphate

ultraviolet

vascular endothelial growth factor

vascular smooth muscle cell

wheat germ agglutinin

wildtype

xanthine oxidase 


\section{General Introduction}

\subsection{Heart failure}

Heart failure is a very common cardiovascular pathology in an ageing and expanding global population. Approximately 37.7 million people globally suffer from heart failure ${ }^{1}$. Studies from the USA and Sweden report a prevalence of more than $2 \%$ in industrialized countries ${ }^{2,3}$. In the USA, more than 550,000 people are diagnosed with heart failure each year and the lifetime risk for developing this syndrome is one in five ${ }^{4}$. By 2030, the prevalence of heart failure in the USA is projected to increase by $46 \%$ to affect more than 8 million people 5 . Besides being common, heart failure is also a severe pathological condition with five-year mortality rates as high as 59\% in men and $45 \%$ in women respectively ${ }^{6}$. This prognosis is poorer than for most cancers with only lung cancer being worse ${ }^{7}$. Heart failure mainly affects older people. More than $50 \%$ of hospitalized patients are 75 years of age or older ${ }^{8}$. Whereas the prevalence is less than $1 \%$ for people younger than 40 years, it is more than $10 \%$ for those aged 80 years and more ${ }^{9}$. Thus, in an ageing population, heart failure is also an economic challenge. Direct costs for heart failure in the USA are meant to increase from 21 billion dollars (2012) to 53 billion dollars in 2030 . In Germany, $1.3 \%$ of direct costs in the healthcare system are attributable to heart failure ${ }^{10}$. Taken together, these data highlight the importance of finding novel therapeutic approaches to target the development of heart failure.

The American College of Cardiology Foundation (ACCF) and American Heart Association (AHA) define heart failure as "a complex clinical syndrome that results from any structural or functional impairment of ventricular filling or ejection of blood"11. Heart failure due to impaired ventricular filling during diastole is often associated with preserved ejection fraction, referred to as $\mathrm{HFpEF}$ (heart failure with preserved ejection fraction). Chapter 3 particularly focuses on $\mathrm{HFpEF}$ with respect to echocardiographic assessment of diastolic function. If systolic function is impaired and the ejection fraction drops to $40 \%$ or less, this is known as HFrEF (heart failure with reduced ejection fraction). Both entities present with similar symptoms in patients such as dyspnea, fatigue and fluid retention. Prevalence and mortality are also comparable, but treatment particularly for HFpEF lacks efficiency ${ }^{12}$. As the ACCF/AHA definition implies, heart failure is a syndrome that can result from a variety of different diseases. The most common cause for heart failure is ischemic heart disease, followed by pathologies such as hypertension, valvular heart 
diseases and cardiomyopathies ${ }^{13}$. In all these diseases, the heart undergoes structural changes that alter cardiac geometry and morphology, as well as functional changes, a process that is collectively called "cardiac remodelling".

\subsection{Cardiac remodelling}

Various stimuli, both physiological and pathological, can induce cardiac remodelling. This includes changes in heart size and shape, which are related to cardiomyocyte hypertrophy and extracellular matrix remodelling. Cardiomyocyte hypertrophy involves the addition of sarcomeres either in series or in parallel. If added in series, cardiomyocytes become longer and the cavity dimension increases, referred to as eccentric remodelling. Concentric remodelling describes increases in cardiomyocyte width with subsequent wall thickening through sarcomere addition in parallel. These two types of remodelling are illustrated in Fig. 1.1.

\section{normal}

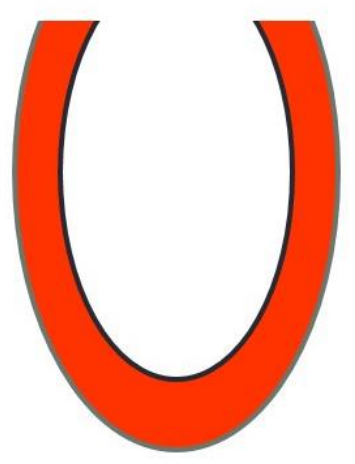

concentric

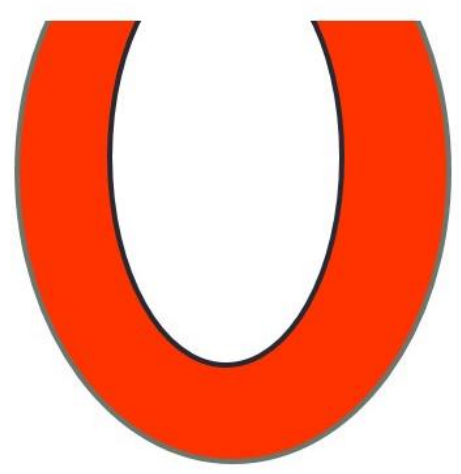

eccentric

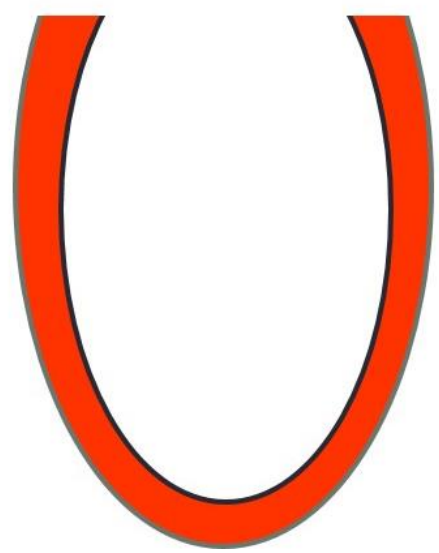

Figure 1.1: Schematic illustration of concentric versus eccentric cardiac remodelling. Compared to the geometry and shape of a normal heart (left), concentric remodelling results in increased wall thickness. This can be associated with mild decreases in left ventricular cavity dimensions (middle). Eccentric remodelling is characterized by left ventricular dilation with or without thinning of ventricular walls (right).

During physiological remodelling, the heart typically undergoes these morphological changes without increased fibrosis, apoptosis or reactivation of the fetal gene program. These changes, however, are commonly seen in pathological remodelling and subsequently lead to the deterioration of cardiac function and the development of heart failure. The underlying 
mechanisms why a heart undergoes physiological or pathological remodelling are still poorly defined. A study from 2006 showed in a mouse model that the duration of cardiac stress is mainly responsible for the amount of hypertrophy, but the nature of the stress determines whether a heart remodels physiologically or pathologically ${ }^{14}$.

Endurance versus strength exercise reflect the different types of cardiac remodelling under physiological conditions. Long-term endurance exercise causes eccentric remodelling as it has been shown to cause significant increases in left ventricular cavity dimensions with only moderate wall thickening. However, a more pronounced increase in wall thickness without increased left ventricular dimension following endurance exercise was reported to be pathological and mainly attributed to primary forms of cardiac hypertrophy such as hypertrophic cardiomyopathy ${ }^{15}$. Isometric strength exercise conditions, such as wrestling, induce predominantly concentric remodelling, as reflected by increases in left ventricular wall thickness rather than dimension ${ }^{16,17}$. On a mechanistic level, most of the available data is based on murine studies. The insulin-like growth factor-1 (IGF-1) has been linked to physiological growth in the heart via activation of the phosphatidylinositol 3-kinase (PI3K)-Akt (protein kinase B/PKB)axis $^{18}$. Although cardiomyocytes retain only a limited ability to proliferate, this was reported to significantly contribute to adaptive cardiac growth under physiological stress. Endurance exercise decreased cardiac levels of the transcription factor C/EBP $\beta$ (CCAAT-enhancer-binding protein $\beta$ ), which subsequently led to increased proliferation of cardiomyocytes ${ }^{19}$. This mechanism was also shown to be protective under pathological pressure overload. Pregnancy and endurance exercise-induced cardiac remodelling are similar in many aspects, which separate them from pathological hypertrophy. Both are rather eccentric than concentric, reversible and show no signs of cardiac fibrosis or expression of fetal genes, as mentioned previously. There is typically a good match between myocardial capillary density and the extent of hypertrophy during physiological remodelling. Whereas endurance exercise causes no impairment in systolic function, some studies report deterioration during pregnancy. However, these findings are based on highly loaddependent parameters (e.g. ejection fraction or fractional shortening), which can be affected by increased preload during pregnancy. Mechanistically, both physiological stimuli are associated with increased cardiac Akt activation ${ }^{20}$.

Under pathological conditions, however, cardiac remodelling has to be seen in a different context. In many pathologies, cardiac remodelling is initially believed to be beneficial and adaptive. However, as the disease progresses, the initial adaptive response eventually ends up in 
the development of heart failure. This transition is typically associated with left ventricular dilation, myocyte apoptosis, myofibroblast proliferation, interstitial fibrosis and a mismatch between capillary density and the extent of hypertrophy. On a molecular level, re-expression of fetal genes as well as impaired excitation-contraction coupling, myofilament function, cell survival and the cellular metabolic state are hallmarks of pathological remodelling leading to heart failure ${ }^{21}$. Cardiac remodelling after myocardial infarction also involves similar changes. The initial removal of necrotic tissue and scar formation are believed to be beneficial and adaptive. However, left ventricular dilation and maladaptive hypertrophy progress, systolic and diastolic wall stress increase with subsequent deterioration of systolic function and development of heart failure. Mechanistically, pathological remodelling is at least partially driven by the activation of neurohormonal systems such as the sympathetic nervous system (SNS) and the renin angiotensin system (RAS). Activation of the SNS and RAS initially help to maintain cardiac output and are therefore an adaptive response. Chronic activation, however, contributes to pathological remodelling including necrosis and fibrosis, and is associated with a poor prognosis in patients ${ }^{22,23}$. From a signalling perspective, catecholamines and angiotensin II (Ang II) are known to activate several protein kinases including protein kinase A (PKA), protein kinase C $(\mathrm{PKC})$, protein kinase D (PKD) and mitogen-activated protein kinases (MAPK). These kinases induce a pro-hypertrophic programme in the heart via regulation of several transcription factors, such as myocyte enhance factor-2 (MEF2) or nuclear factor of activated T-cells (NFAT), and thereby contribute to long-term maladaptation of the heart ${ }^{24}$.

\subsection{Pressure versus volume haemodynamic load}

Haemodynamic load is an important pathological stressor for most conditions that induce cardiac remodelling, and is of particular interest in this thesis. It may be divided into pressure and volume overload. Pressure overload involves an increase in afterload, which can be defined as "all of the factors that contribute to total myocardial wall stress during systolic ejection" 25 . Under healthy conditions, this may be estimated by the mean aortic blood pressure. Volume overload increases mainly preload, which can be defined as "all the factors that contribute to passive ventricular wall stress at the end of diastole" ${ }^{25}$. Both pressure and volume overload increase cardiac work which, by the law of Laplace, is directly proportional to ventricular pressure (increased in response to increased afterload) and also directly proportional to ventricular volume (increased in response to increased volume or preload). Classical pressure overload diseases with increased afterload are 
aortic stenosis and hypertension, which cause concentric remodelling and hypertrophy. Under pathological volume overload, such as in aortic/mitral regurgitation or ventricular septal defects, cardiomyocytes are stretched during diastole, which causes eccentric remodelling and hypertrophy. According to the law of Laplace, concentric remodelling reduces wall stress as a result of the increase in wall thickness. With eccentric remodelling, there is no such decrease in wall stress but on the other hand, there is an increase in stroke volume due to the Frank-Starling law; i.e. an increased recruitment of preload reserve. These differences were already reported in 1975 by Grossman et al. ${ }^{26}$. In patients with aortic stenosis, both left ventricular systolic and diastolic wall stress were normalized despite elevated systolic and diastolic pressure levels. This was attributed to the increase in wall thickness as the hallmark of concentric remodelling, which was believed to be an adaptive response. Patients with volume overload-associated diseases also displayed normal systolic wall stress, but increased diastolic wall stress. From a haemodynamic point of view, eccentric remodelling following volume overload has been suggested to be maladaptive since wall stress is increased. However, based on experience, clinical outcomes appear to be better in patients with volume overload-associated heart diseases compared to pressure overload. Unfortunately, no human study has investigated this so far, presumably due to the difficult comparability of both stresses. Outcomes can also be studied and assessed in a more controlled manner using animal models. In a recent murine study that compared models of pressure and volume overload, despite a similar induction of left ventricular wall stress, pressure overload was associated with a more detrimental phenotype than volume overload ${ }^{27}$. This included increased cardiac fibrosis, cardiac dysfunction and higher mortality following pressure overload. Changes induced by chronic volume overload, especially in the early stages, were similar to changes during physiological remodelling including preserved systolic function and an absence of interstitial fibrosis. These differences between pressure and volume overload were at least partially due to different signalling under both stress conditions in the heart, which emphasizes the complexity of cardiac remodelling and its implication in cardiovascular diseases. The differences in cardiac responses to pressure and volume overload will be addressed throughout this thesis and investigated under different aspects in Chapters 3, 4 and 5.

Heart failure therapy focuses on reversing or at least attenuating pathological remodelling. Inhibiting the RAS using angiotensin converting enzyme (ACE)-inhibitors or direct angiotensin II receptor-blockers is well-established as being sufficient and effective in heart failure therapy ${ }^{28}$, 29 . Addition of a mineralocorticoid receptor antagonist, such as spironolactone or eplerenone, to 
the standard therapy also reduces the risk of morbidity and mortality amongst patients with severe heart failure ${ }^{30,31}$. A recent study reported the benefits of using a novel angiotensin II antagonist named LCZ696 ${ }^{32}$. This drug additionally inhibits neprilysin, which is an endopeptidase that mediates RAS overactivation. Using LCZ696 was shown to be superior to the ACE-inhibitor enalapril in reducing risks of death and hospitalization in heart failure patients. Besides pharmacological treatment, resynchronization therapy using implantable devices also belongs to the standard therapy as it improves survival in patients with severe heart failure ${ }^{33}$. Despite the optimal use of such therapies, mortality and morbidity remain high in heart failure. Whether therapies might have different effects in pressure versus volume overload has not been extensively studied.

Taken together, a more detailed understanding of cardiac remodelling under different stress conditions and the transition to heart failure is needed for developing novel therapeutic options.

\subsection{Cardiac metabolism in heart failure}

The heart is the organ with the highest energy demand in the human body. Complete ATP turnover approximately occurs every ten seconds since the heart has both a relatively low ATP content $(5 \mu \mathrm{mol} /$ gram wet weight) and very high rates of ATP hydrolysis $(0.5 \mu \mathrm{mol} \mathrm{x} \mathrm{g}$ wet weight $^{-1} \mathrm{x} \mathrm{s}^{-1}$ ). Under normoxic conditions, approximately 95\% of the ATP derives from oxidative phosphorylation in the mitochondria with the remaining 5\% coming mainly from glycolysis. Oxidative phosphorylation requires $\mathrm{NADH}$ as an electron donor to generate proton motive force to drive F1 ATP synthase; this NADH is generated in the tricarboxylic acid (TCA) cycle. Approximately two thirds of the energy is needed for contraction, the rest for various ion pumps and other cellular processes that require ATP. Besides ATP, phosphocreatine represents an additional energy pool in the heart. It serves as an ATP transporter and buffer. In the mitochondria, a phosphate group can be transferred from ATP to creatine by the mitochondrial creatine kinase. Phosphocreatine can easily diffuse through the mitochondrial membrane due to its smaller molecular weight. In the cytosol, ATP can be re-generated through phosphate-transfer from phosphocreatine to ADP, catalyzed by the cytosolic creatine kinase ${ }^{34,35}$.

Under healthy conditions, approximately 70-90\% of cardiac energy derives from fatty acid oxidation, with the remaining 10-30\% mainly coming from glucose and lactate oxidation. Fatty acids enter the cardiomyocytes and are then esterified to coenzyme A $(\mathrm{CoA})$ to produce fatty acyl-CoA. Carnitine palmitoyltransferase 1 (CPT1) converts fatty acyl-CoA to long-chain 
acylcarnitine, which can enter the mitochondria. In the mitochondria, CPT2 converts long-chain acylcarnitine back to fatty acyl-CoA, which then enters $\beta$-oxidation to produce acetyl-CoA, which enters the TCA cycle. Glucose in the heart comes from either intracellular glycogen stores or exogenous glucose uptake via glucose transporters (GLUT). Glucose is phosphorylated to glucose-6-phosphate, which can enter glycolysis to produce pyruvate. Pyruvate can then either be converted to lactate in the cytosol or oxidized at the mitochondrial membrane to acetyl-CoA to enter the TCA cycle. Additionally, glucose-6-phosphate can be utilized in the pentose phosphate pathway (PPP) to produce NADPH or the hexosamine biosynthetic pathway (HBP) for the production of UDP-N-acetylglucosamine (GlcNAc), an important monosaccharide donor for OGlcNAcylation of several proteins ${ }^{34}$. Acetyl-CoA is a common product from both fatty acid and glucose oxidation, which can enter the TCA cycle to produce GTP (or ATP), $\mathrm{CO}_{2}$ and the reducing equivalent $\mathrm{NADH}$. In the TCA cycle, intermediate products are permanently removed and used for several biosynthetic pathways. Acetyl-CoA-independent production and replacement of these intermediates in the TCA cycle is therefore an important mechanism, called anaplerosis ${ }^{34}$.

In heart failure, cardiac energy metabolism changes significantly. Reduction in cardiac phosphocreatine/ATP (PCr/ATP) ratio is a hallmark of impaired energy metabolism and correlates with the degree of heart failure. It is also a predictor of both total and cardiovascular mortality in patients with dilated cardiomyopathy ${ }^{36}$. Although some studies show contradictory results, it is generally accepted that utilization of fatty acids in the heart decreases during the development of heart failure. Using an invasive technique, patients with dilated cardiomyopathy displayed a decrease in both fatty acid uptake and oxidation in the heart compared to controls ${ }^{37}$. Additionally, fatty acid uptake was negatively correlated with left ventricular chamber enlargement suggesting a decrease in fatty acid utilization during heart failure Another study reported similar findings using positron emission tomography (PET) for measurements of myocardial fatty acid utilization and oxidation ${ }^{38}$. Both parameters were significantly reduced in patients with idiopathic dilated cardiomyopathy compared with healthy controls. This is in line with decreased cardiac mRNA and protein expression levels of fatty acid oxidation enzymes in human and animal heart failure samples compared to respective controls ${ }^{39}$. Data on glucose metabolism in heart failure is much more contradictory and diverse. This might partially be explained by fundamental differences in terms of metabolic changes between humans and rodents, where most of these studies have been carried out. The precise metabolic properties are 
also dependent on the severity and nature of cardiac stress such as pressure versus volume overload. Those two haemodynamic stresses potentially induce very different metabolic profiles in the heart. In an animal model of pressure overload, glucose oxidation by pyruvate dehydrogenase (PDH) was shown to be unchanged despite increased glycolysis ${ }^{40}$. This finding was in line with increased anaplerotic flux into the TCA cycle, generally suggesting increased anaplerosis in pressure overload. It is possible that increased anaplerosis contributes to the mismatch between glycolysis and glucose oxidation, which is commonly seen in pressure overload. Whether this is specific for pressure overload or also occurs under volume overload is currently unknown. In Chapter 4, this is therefore further studied with particular focus on pressure versus volume overload-induced changes in cardiac metabolism.

Modulation of cardiac metabolism has long been a target for novel therapies in heart failure. But since the data on the role of cardiac metabolism in heart failure development are controversial and contradictory, the same accounts for potential therapies. Reducing fatty acid oxidation using the CPT1-inhibitor perhexiline has been shown to improve the phosphocreatine/ATP ratio, diastolic function and exercise capacity in symptomatic patients suffering from hypertrophic cardiomyopathy ${ }^{41}$. However, studies in both animals and humans demonstrated the importance of cardiac fatty acid utilization in heart failure. Treatment of healthy controls with acipimox, a niacin derivate known to reduce lipid levels, reduced free fatty acid serum levels, decreased cardiac work and oxidative metabolism with preserved myocardial efficiency ${ }^{42}$. In patients with idiopathic dilated cardiomyopathy, however, acipimox treatment was associated with decreased cardiac work, unchanged oxidative metabolism and deterioration of myocardial efficiency. These findings suggest the need for fatty acids in the failing heart for optimal function. Using dichloroacetate (DCA) to activate PDH through inhibition of pyruvate dehydrogenase kinase (PDK) results in inhibition of free fatty acid metabolism, but increases in glucose and lactate consumption in the heart. One study reported that short-term treatment of heart failure patients with DCA improved cardiac function, another study demonstrated no beneficial effects ${ }^{43,44}$.

Taken together, various changes in metabolism seem to play an important role in cardiac remodelling and its transition to heart failure. However, these changes are likely to be different depending on the kind of cardiac stress. Thus, the evidently different cardiac responses to pressure and volume overload with respect to remodelling might also involve distinctive metabolic changes. Assessing, identifying and comparing these under both haemodynamic stress conditions will help to get a more detailed understanding of how cardiac remodelling is 
influenced by metabolism. This knowledge could help to develop new therapeutic strategies, to attenuate the progression towards heart failure. This will be further discussed in Chapter 4 .

\subsection{Reactive oxygen species (ROS) in heart failure}

Reactive oxygen species (ROS), including superoxide anion $\left(\mathrm{O}_{2}^{-}\right)$, hydrogen peroxide $\left(\mathrm{H}_{2} \mathrm{O}_{2}\right)$ and hydroxyl radicals $(\mathrm{OH})$ are either by-products from cellular aerobic respiration through mitochondrial leakage or generated by certain enzymes. Amongst these enzymes are the xanthine oxidase (XO), monoamine oxidase (MAO), uncoupled NO synthases (NOS) and NADPH oxidases (Nox). One electron reduction of molecular oxygen $\left(\mathrm{O}_{2}\right)$ forms $\mathrm{O}_{2}{ }^{-}$, which typically dismutates to the more stable $\mathrm{H}_{2} \mathrm{O}_{2} . \mathrm{O}_{2}$ can also be used by the NOS to produce nitric oxide (NO), a reactive nitrogen species (RNS). Together with $\mathrm{O}_{2}{ }^{-}$, NO can rapidly form peroxynitrite $\left(\mathrm{ONOO}^{-}\right)$, an additional reactive species. All these different molecules contribute to "redox signalling", a term which describes altered cellular signalling components through oxidation or reduction respectively ${ }^{45}$. One of the most common mechanisms in redox signalling is the direct oxidation of cysteine thiols, causing intra- or intermolecular disulfide formation. This can lead to changes in conformation, stability and function of affected proteins. An example for this mechanism contributing to cellular signalling is the inhibition of protein tyrosine phosphatases (PTP). The enzymatic activity of PTPs depends on a cysteine residue, located in the active site of the enzyme. ROS-mediated oxidation of this cysteine results in inactivation of the enzyme. This causes increases in tyrosine phosphorylation levels, an important regulatory mechanism in several cellular processes ${ }^{46}$.

Various relevant proteins in cardiac physiology and pathology are known to be regulated through redox signalling. Amongst these are calcium/calmodulin-dependent protein kinase II (CaMKII), protein kinase A (PKA) and protein kinase $\mathrm{G}(\mathrm{PKG})$, all of which are redox-activated, and ryanodine receptor 2 (RyR2), sarcoplasmic/endoplasmic reticulum calcium ATPase-2 $\alpha$ (SERCA$2 \alpha$ ), GTPases, antioxidant proteins and histone deacetylases, whose function is modulated by ROS $^{47}$. In the past, ROS production has been exclusively linked to pathological processes. An important aspect in this context is the amount of ROS being produced. Excessive amounts are detrimental through unspecific redox modification of macromolecules while redox signalling may also be pathological. However, there is increasing evidence that ROS also exert physiological functions in the cardiovascular system. For instance, during tachycardia, ROS production in the heart has been shown to modify RyR2 through S-glutathionylation, leading to a 
faster, beneficial calcium release during increased cardiac activity ${ }^{48}$. ROS can also play a physiological role in blood pressure regulation. Under normal conditions, $\mathrm{H}_{2} \mathrm{O}_{2}$ can activate the PKG-I $\alpha$ independently of NO and cGMP, which causes vasodilation. In a transgenic mouse model, amino acid substitution created a "redox-dead" PKG-I $\alpha$ version, which could not be activated by $\mathrm{H}_{2} \mathrm{O}_{2}{ }^{49}$. This resulted in hypertension in vivo, indicating a physiological role for redox activation of PKG. Low levels of $\mathrm{H}_{2} \mathrm{O}_{2}$ have also been shown to increase cardiomyogenesis of embryonic stem (ES) cells and induced proliferation of cardiomyocytes derived from ES cells and neonatal mice, suggesting a physiological role for ROS in cardiac development ${ }^{50}$.

Oxidative stress describes the imbalance of ROS production and antioxidant defense mechanisms including enzymes like superoxide-dismutase, catalase, peroxiredoxins and thioredoxin as well as glutathione as main redox buffer and vitamins E and C. Oxidative stress causes pathological redox signalling and is known to contribute to heart failure development. In patients with coronary artery disease, oxidative stress in the heart was positively correlated with left ventricular dilation $^{51}$. Mechanistically, this was associated with increased matrix metalloproteinase (MMP) levels, which might have contributed to the progression of left ventricular dilation under increased oxidative stress. Both ROS-generating enzymes XO and MAO have been implicated in cardiac pathology. Inhibition of XO significantly improved cardiac function of hypertensive rats, and mice with a dominant negative MAO-A were protected from pressure overload ${ }^{52,53}$. ROS can also contribute to impaired calcium handling. In an experimental heart failure model, increased $\mathrm{Ca}^{2+}$ leak from the sarcoplasmic reticulum (SR) was attributed to direct oxidation and irreversible activation of RyR2 by increased $\operatorname{ROS}^{54}$. The duration of cardiac stress and subsequent ROS production seem to be important in altering calcium handling. Acute irradiation of the heart increases cardiac ROS levels and initially systolic force with increased calcium transient amplitudes ${ }^{55}$. However, under chronic irradiation, calcium transient amplitudes as well as SR calcium load decreased together with a decline in systolic force. This was shown to be due to ROS-dependent CaMKII activation and subsequent hyper-phosphorylation of RyR2. ROS production can also contribute to cardiac arrhythmias as it has been linked to the development of atrial fibrillation in both humans and experimental animal models ${ }^{56,57}$. Furthermore, oxidative stress can cause cardiomyocyte apoptosis. Chronic stimulation with angiotensin II (Ang II) induces apoptosis of cardiomyocytes via ROS-dependent sustained activation of CaMKII ${ }^{58}$. Under chronic $\beta$-adrenergic stimulation, increased apoptosis was induced by activation of the 
mitochondrial death pathway through a ROS/c-Jun NH2 terminal-kinase (JNK)-dependent mechanism ${ }^{59}$.

Since ROS exert both physiological and pathological functions in the heart, they remain a challenging target for potential therapeutic approaches. Two main strategies are under current investigation: Reducing ROS or increasing antioxidants. Treatment of hypertensive rats with MitoQ, a potent mitochondria-targeted antioxidant, has been shown to attenuate the development of hypertension, improves endothelial function and reduces cardiac hypertrophy ${ }^{60}$. It was also shown to improve cardiac function, cell death and mitochondrial damage after ischemiareperfusion injury in rats ${ }^{61}$. This compound is currently being tested in clinical trials. Nox inhibition is another current approach in drug therapy development. As there is a specific focus on Nox enzymes throughout this thesis, the next section will give a brief overview about the main two Nox isoforms in the heart.

\subsection{NADPH oxidases in the heart}

Nox enzymes use NADPH as electron donor to reduce $\mathrm{O}_{2}$ to $\mathrm{O}_{2}^{-}$and $\mathrm{H}_{2} \mathrm{O}_{2}$ respectively. With Nox2 (also referred to as gp91 ${ }^{\text {phox }}$ ) being the first one to be discovered, six more Nox family members are currently known: Nox1, Nox3, Nox4, Nox5, Dual oxidases 1 (Duox1) and 2 $(\text { Duox } 2)^{62}$. Despite several similarities, these isoforms differ in structure, regulation and localization. The membrane-spanning Nox2 protein forms a heterodimer with the small subunit $\mathrm{p} 22^{\text {phox }}$. This heterodimer requires binding of cytosolic subunits $\mathrm{p} 40^{\mathrm{phox}}, \mathrm{p} 47^{\mathrm{phox}}, \mathrm{p} 67^{\mathrm{phox}}$ and Rac for stimulation of its catalytic activity. This activation occurs in response to stimuli such as Ang II, endothelin-1, growth factors, cytokines, metabolic factors and mechanical forces. Nox1 forms a heterodimer with $\mathrm{p} 22^{\text {phox }}$, and for activation requires binding to Rac1 as well as NOXO1 and NOXA1, which are homologues of $\mathrm{p} 47^{\text {phox }}$ and $\mathrm{p} 67^{\mathrm{phox}}$. Nox3 can function just as a heterodimer with $\mathrm{p} 22^{\text {phox }}$, but its activity is stimulated by binding of $\mathrm{p} 47^{\mathrm{phox}}$ and $\mathrm{p} 67^{\mathrm{phox}}$. Nox4 is different with respect to its regulation. It forms a heterodimer with $\mathrm{p} 22^{\text {phox }}$, but does not require any additional subunits for activation. It is constitutively active and therefore regulated via its abundance ${ }^{63}$. In contrast to other Nox enzymes, Nox4 has also been shown to produce $\mathrm{H}_{2} \mathrm{O}_{2}$ rather than $\mathrm{O}_{2}^{-}$. This is at least partially attributed to its histidine-rich extracytosolic loop (Eloop), which accelerates spontaneous dismutation of $\mathrm{O}_{2}{ }^{-}$to $\mathrm{H}_{2} \mathrm{O}_{2}{ }^{64}$. Nox5 is the most divergent from Nox2. It is found in humans, not rodents, does not require p22 $2^{\text {phox }}$ binding and contains EFhand binding calcium motifs. Nox1, Nox2, Nox4 and Nox5 are expressed throughout the 
cardiovascular systems with different cellular distribution: Nox1 is expressed mainly in vascular smooth muscle cells (VSMC); Nox2 in endothelial cells, fibroblasts, cardiomyocytes and inflammatory cells; Nox4 in endothelial cells, fibroblasts, cardiomyocytes and VSMCs; Nox5 in human endothelial cells, fibroblasts and VSMCs ${ }^{65}$. Key features with respect to regulation and localization of these four Nox-isoforms are summarized in Fig. 1.2.
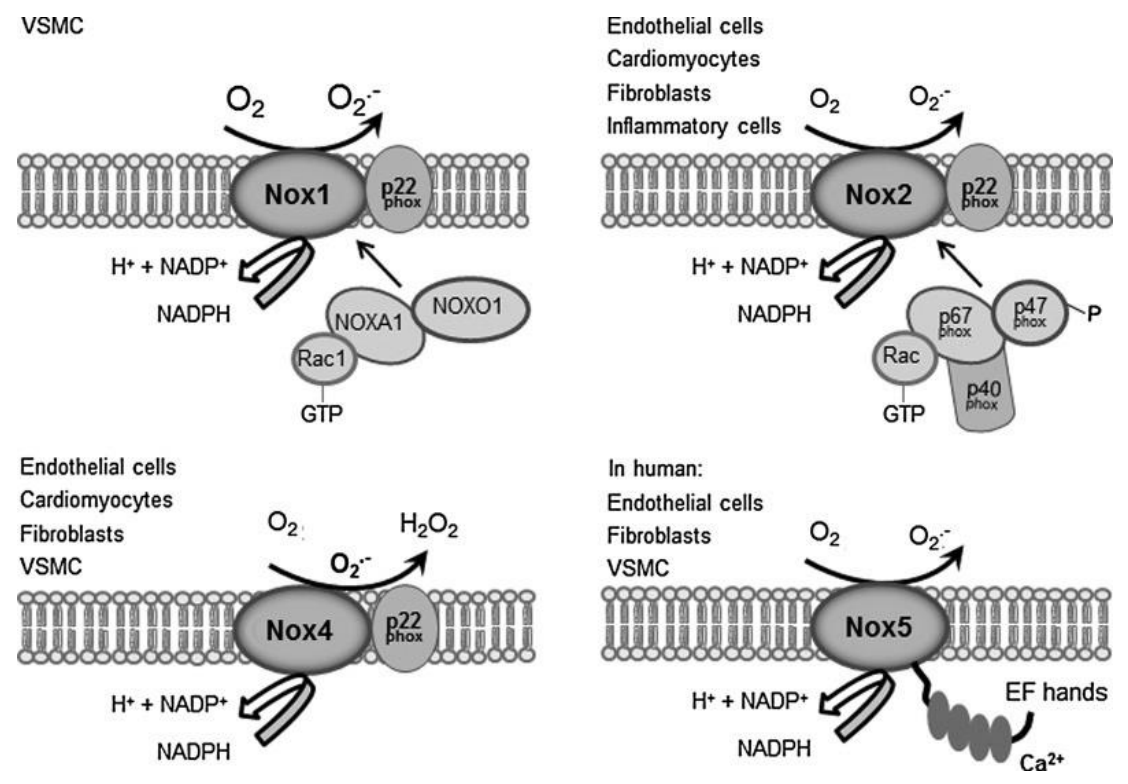

Figure 1.2: Schematic illustration of NADPH oxidases with respect to their structure, regulation and cellular distribution (Adapted from Antioxid Redox Signal 2013; 18(9): 1024-1041). Nox1, Nox2, Nox4 and Nox5 are

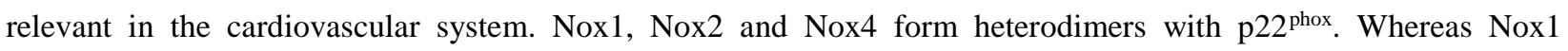
requires activation through binding of Rac1, NOXA1 and NOXO1, Nox2 is activated via Rac, p40 phox p47 phox and p6 $7^{\text {phox }}$. Both Nox-isoforms produce $\mathrm{O}_{2}^{-}$through electron transfer from NADPH to $\mathrm{O}_{2}$. Nox4 does not require activation, is constitutively active and produces $\mathrm{H}_{2} \mathrm{O}_{2}$ rather than $\mathrm{O}_{2}{ }^{-}$. Nox5 is only found in humans, does not bind p2 $2^{\text {phox }}$ and contains EF-hand binding calcium motifs. Cellular distributions are listed on the top left for each isoform scheme. Abbreviations: GTP (guanosine triphosphate), NAPDH (nicotinamide adenine dinucleotide phosphate hydrogen), NOXO (NADPH oxidase organizer), NOXA (NADPH oxidase activator), phox (phagocyte oxidase), VSMC (vascular smooth muscle cell).

As depicted in Fig. 1.2, the two main isoforms in cardiomyocytes are Nox2 and Nox4. Both have been implicated in physiological and pathological processes in the heart. Nox 2 has recently been reported to differentially regulate calcium handling, excitation-contraction coupling and global contractile function in response to neurohormonal versus chronic mechanical stress (e.g. Ang II and chronic pressure overload). Zhang et al. demonstrated short-term increases in systolic 
function under Ang II stimulation in cardiomyocyte-specific Nox2 overexpressing mice compared to WT littermates ${ }^{66}$. Under chronic pressure overload, however, systolic function was reduced through Nox2 overexpression, depicting different roles for ROS in cardiac pathophysiology under acute and chronic stress respectively. In addition, many studies report Nox2-dependent detrimental changes in cardiac remodelling under various pathological stimuli. After Ang II stimulation, Nox2-null mice were protected from increased fibrosis and systolic dysfunction compared to WT littermates despite similar increases in blood pressure and left ventricular hypertrophy ${ }^{67,68}$. However, without affecting blood pressure using sub-pressor doses of Ang II, Nox2-null mice were also protected from hypertrophy compared to WT littermates ${ }^{69}$. Thus, Nox2 seems to contribute to cardiac hypertrophy exclusively under agonist but not mechanical stimulation, considering that an increased blood pressure induces mechanical stress. Nox2 is also implicated in maladaptive cardiac remodelling after myocardial infarction (MI). Animal studies in mice demonstrate a detrimental role for Nox2 in post-MI remodelling, as Nox2 -null mice are protected from left ventricular dilation, hypertrophy, dysfunction and fibrosis compared to WT littermates ${ }^{70}$. A potential involvement of Nox2 in post-MI remodelling was also reported in humans as heart tissue from patients with acute myocardial infarction showed increased Nox 2 levels in cardiomyocytes ${ }^{71}$. Despite a few studies showing increased contractility through Nox2, the majority of studies demonstrate a rather detrimental role for Nox 2 in the heart under different stress conditions. This does not apply to Nox4. Many studies report beneficial and protective roles for Nox4 in the heart. As previously mentioned, low levels of $\mathrm{H}_{2} \mathrm{O}_{2}$ increase cardiomyogenesis. This finding is supported by the observation that Nox4 overexpressing cardiomyocytes show an increased cell cycling capacity compared to WT littermates in vivo ${ }^{72}$. This was due to Nox4-mediated increases in $\mathrm{H}_{2} \mathrm{O}_{2}$ production. In a mouse model of chronic pressure overload, cardiomyocyte-derived Nox4 has also been shown to protect from maladaptive remodelling and systolic dysfunction through increased angiogenesis via HIF-1 $\alpha$ (hypoxiainducible factor $1 \alpha$ ) stabilization ${ }^{73}$. This finding is in line with a human study by Moreno et al., which reported decreased levels of Nox4 in cardiac tissue from patients with aortic stenosis $(\mathrm{AS})^{74}$. In these patients, Nox4 levels correlated with capillary density and inversely correlated with apoptosis in the heart. In addition, Nox4 levels showed a positive correlation with systolic function in AS patients. Therefore, the decrease in Nox4 levels seen in AS patients might be contributing to maladaptive cardiac remodelling and deterioration of cardiac function suggesting a protective role for endogenous Nox4 in the heart. These studies focus on pressure overload- 
induced cardiac remodelling, whereas the potential role for Nox4 in eccentric remodelling following volume overload is unknown. This is extensively studied in Chapter 5 of this thesis.

Taken together, the differences between Nox 2 and Nox4 with respect to their physiological and pathological roles in cardiac remodelling once again highlight the complexity of redox signalling in the heart. 


\subsection{Aims}

Haemodynamic stress, including pressure and volume overload, is evident in several cardiovascular diseases such as valvular heart diseases, ventricular septal defects and hypertension. Whereas pressure overload leads to rapid deterioration of systolic function, maladaptive cardiac remodelling and increased mortality in rodent models, volume overload can be compensated for a long period of time with an initial phenotype similar to physiological remodelling. As the underlying mechanisms for this beneficial cardiac adaptation in response to chronic volume overload are currently unknown, the aims of this thesis were: (i) to develop and validate state-of-the-art echocardiographic parameters to characterize systolic and diastolic function in a murine model of volume overload (aortocaval fistula model) as compared to other forms of haemodynamic stress (e.g. pressure overload induced by abdominal aortic banding); (ii) to use this model to investigate changes in cardiac glucose metabolism in volume versus pressure overload with a novel in vivo ${ }^{13} \mathrm{C}$-labelling methodology; (iii) to study the role of Nox4 in the development of eccentric remodelling during volume overload in mice.

This work aimed to provide new physiological, metabolic and mechanistic insights into changes occurring in the heart, particularly in response to volume overload, which may be more generally important in understanding cardiac remodelling and developing new therapeutic approaches. 


\section{Material and Methods}

\subsection{Gene-modified mice}

Mice were kept in a temperature and humidity controlled animal facility ("Biological services unit” at Denmark Hill Campus, King's College London) with 12-hour light/dark cycles and free access to food and water. Studies were conducted in accordance with the UK Home Office Guidance on the Operation of the Animals (Scientific Procedures) Act, 1986.

Global Nox4-null mice (Nox4 $4^{-/}$) were generated by targeted deletion of the translation initiation site, exon 1 and exon 2 of the Nox4-gene as described previously ${ }^{73}$. Mice were backcrossed $>10$ generations into a $\mathrm{C} 57 \mathrm{Bl} / 6$ background before heterozygous mice were paired to produce Nox $4^{-/}$mice and wildtype (WT) littermates. Nox $4^{-/-}$-mice were born in the expected Mendelian ratio and showed no basal phenotype with respect to cardiac size and function. Successful deletion was confirmed by the absence of Nox4-protein in heart lysates together with a small reduction in $\mathrm{H}_{2} \mathrm{O}_{2}$ levels compared to WT littermates. Protein levels of Nox2 and p22 phox in the heart remained unchanged ${ }^{73}$. For generation of cardiomyocyte-specific Nox4 overexpressing mice (Nox4tg), full length mouse Nox4-cDNA was cloned downstream of the $\alpha$-myosin heavy chain promotor, as previously described ${ }^{73}$. This construct was introduced into the mouse genome. After backcrossing for more than 10 generations into a C57B1/6 background, heterozygous transgenic male mice were paired with WT females. Nox4tg-mice displayed increases of approximately 8 fold in Nox4 protein and 1.5 fold in $\mathrm{H}_{2} \mathrm{O}_{2}$ production in the heart compared to WT littermates. Cardiac protein levels of $\mathrm{p} 22^{\text {phox }}$, which forms a heterodimer with Nox4, was also 2.5 fold increased. Levels of Nox2 and $\mathrm{O}_{2}^{-}$production were unaffected. Cardiac mass was slightly increased in aged Nox4tg-mice without any cardiac dysfunction, fibrosis and apoptosis suggesting no detrimental consequences through Nox4 overexpression ${ }^{73}$.

For pure WT experiments, female and male C57Bl/6 mice were purchased from Harlan Laboratories (UK).

\subsection{Genotyping}

For genotyping of the Nox $4^{-/-}$- and Nox4tg-mouse lines, DNA was isolated as follows: murine ear punches were incubated with $300 \mu \mathrm{NaOH}(50 \mathrm{mM})$ at $95^{\circ} \mathrm{C}$ for 30 minutes. Samples were vortexed before $30 \mu \mathrm{l}$ of Tris- $\mathrm{HCl}(1 \mathrm{M}, \mathrm{pH}$ 8.0) were added, mixed, and stored at room 
temperature for another 30 minutes. Small volumes $(2 \mu \mathrm{l})$ of this solution could directly be used for polymerase chain reactions (PCR), which also included REDTaq ${ }^{\circledR}$ ReadyMix ${ }^{\mathrm{TM}}$ PCR Reaction Mix (Sigma-Aldrich), water and specific primers. PCR products were visualized by UV detection using agarose gel electrophoresis (1.5\% agarose w/v and $0.005 \%$ ethidium bromide v/v in Tris-acetate-EDTA buffer (TAE)).

In the Nox $4^{-/-}$-line, primers were designed to identify WT, heterozygous and knockout (KO) animals depending on the sizes of different PCR products detected. In the Nox4tg-line, specific primers were used to amplify the transgene with GAPDH as internal control. The exact primer sequences and conditions can be found in Tables 2.1 and 2.2.

\begin{tabular}{|l|l|}
\hline Primer sequences: & \\
\hline Nox4WT_forward (5'-3') & GTTGCTGGCTTCTGCTTCTT \\
Nox4KO_forward (5'-3') & AAGCTTCCGATTCCATTCT \\
Nox4WT/KO_reverse (5'-3') & CTTTGTGTGGTTGCTTAGGAGA \\
\hline PCR reaction components: & total volume of $25 \mu \mathrm{I}$ Nox4WT/KO reaction mix: \\
\hline $\begin{array}{l}\text { RedTaq }{ }^{\circledR} \text { ReadyMix } \\
\text { Primers: }\end{array}$ & $12.5 \mu \mathrm{l}$ \\
Nox4WT_forward $(10 \mu \mathrm{M})$ & $1 \mu \mathrm{l}$ \\
Nox4KO_forward $(10 \mu \mathrm{M})$ & $0.5 \mu \mathrm{l}$ \\
Nox4WT/KO_reverse $(10 \mu \mathrm{M})$ & $0.5 \mu \mathrm{l}$ \\
$\mathrm{H}_{2} \mathrm{O}$ & $8.5 \mu \mathrm{l}$ \\
DNA template & $2 \mu \mathrm{l}$ \\
\hline PCR conditions: & \\
\hline Initialisation & $94^{\circ} \mathrm{C}, 5$ Minutes \\
Denaturation (40 cycles) & $94^{\circ} \mathrm{C}, 30$ Seconds \\
Annealing (40 cycles) & $60^{\circ} \mathrm{C}, 30$ Seconds \\
Elongation (40 cycles) & $72^{\circ} \mathrm{C}, 30$ Seconds \\
Final Elongation & $72^{\circ} \mathrm{C}, 7$ Minutes \\
\hline
\end{tabular}

Table 2.1: Genotyping protocol for the global Nox4-null mouse line $\left(\mathrm{Nox}^{-/-}\right)$. 


\begin{tabular}{|l|l|}
\hline Primer sequences: & \\
\hline Nox4tg_forward $\left(5^{\prime}-3^{\prime}\right)$ & CCAGAATGAGGATCCCAGAA \\
Nox4tg_reverse $\left(5^{\prime}-3^{\prime}\right)$ & ACCACCTGAAACATGCAACA \\
Gapdh_forward $\left(5^{\prime}-3^{\prime}\right)$ & CCTAGACAAAATGGTGAAGG \\
Gapdh_reverse $\left(5^{\prime}-3^{\prime}\right)$ & GACTCCACGACATACTCAGC \\
\hline PCR reaction components: & total volume of $25 \mu \mathrm{l}$ Nox4tg/GAPDH reaction mix: \\
\hline RedTaq ${ }^{\circledR}$ ReadyMix & $12.5 \mu \mathrm{l}$ \\
Primers: & \\
Nox4tg_forward $(10 \mu \mathrm{M})$ & $0.5 \mu \mathrm{l}$ \\
Nox4tg_reverse $(10 \mu \mathrm{M})$ & $0.5 \mu \mathrm{l}$ \\
Gapdh_forward $(10 \mu \mathrm{M})$ & $0.5 \mu \mathrm{l}$ \\
Gapdh_reverse $(10 \mu \mathrm{M})$ & $0.5 \mu \mathrm{l}$ \\
H2O & $8.5 \mu \mathrm{l}$ \\
DNA template & $2 \mu \mathrm{l}$ \\
\hline PCR conditions: & \\
\hline Initialisation & $94^{\circ} \mathrm{C}, 5$ Minutes \\
Denaturation (35 cycles) & $94^{\circ} \mathrm{C}, 30$ Seconds \\
Annealing (35 cycles) & $56^{\circ} \mathrm{C}, 45$ Seconds \\
Elongation (35 cycles) & $72^{\circ} \mathrm{C}, 1$ Minute \\
Final Elongation & $72^{\circ} \mathrm{C}, 7$ Minutes \\
\hline
\end{tabular}

Table 2.2: Genotyping protocol for the cardiomyocyte-specific Nox4 overexpressing mouse line (Nox4tg).

\subsection{Murine volume overload model}

The aortocaval fistula (Shunt) is an established model to induce volume overload in mice ${ }^{75}$. Mice aged 7-11 weeks were anesthetized using 1.5\% isoflurane insufflation. Finadyne s.c. (100 $\mu \mathrm{g}$ diluted in $100 \mu \mathrm{l}$ sterile saline per mouse; MSD Animal Health, Milton Keynes, UK) and Vetergesic i.m. (0.1 mg/kg; Alstoe Animal Health, York, UK) were injected for post-surgical pain relief. For Shunt operation, a longitudinal abdominal incision was made, the intestine put aside and the aorta as well as the inferior vena cava (IVC) were dissected free from any surrounding tissue. The aorta was clamped just above the renal arteries and punctured with a 23gauge needle in an infrarenal position, followed by penetration of the needle into the IVC. Since aorta and IVC share the same middle wall, an aortocaval fistula was created. After removing the needle, the external hole in the aorta was closed using cyanoacrylate glue (Krazy glue, Elmer's Products, USA). Depending on the oxygen status of the animal, in most cases mixing of oxygenated blood from the abdominal aorta into the IVC could be observed. The abdomen was then closed and the mice were kept on a heating plate until full recovery from anesthesia. Sham 
animals underwent the same procedure except for the puncture of the vessels. A schematic illustration of this surgery can be found in Fig. 2.1.

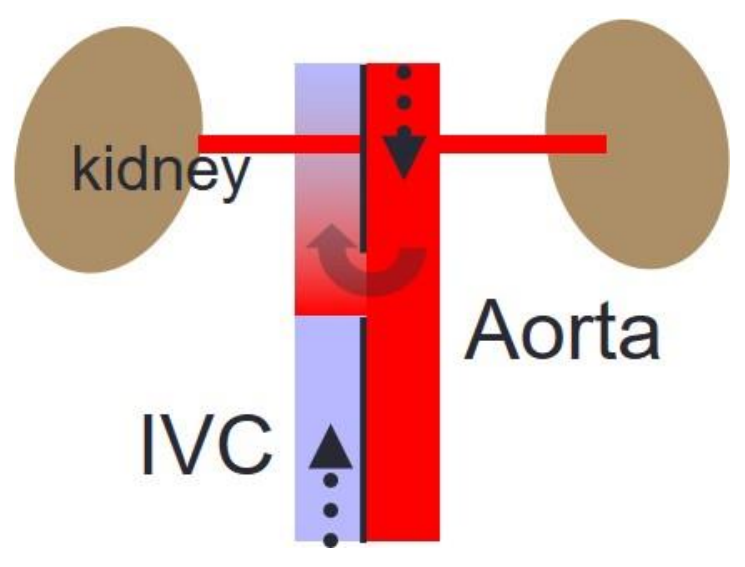

Figure 2.1: Schematic illustration of the aortocaval fistula (Shunt) surgery. A fistula between the aorta and the inferior vena cava (IVC) is created in an infrarenal position to allow blood flow directly from the arterial into the venous system and thereby increasing cardiac volume load. The arrows indicate blood flow, the colour change in the upper IVC is due to mixing of oxygenated, red and deoxygenated, blue blood through the fistula.

\subsection{Murine pressure overload models}

Transverse aortic constriction (TAC) and abdominal aortic banding (AAB) are two commonly used models to induce pressure overload in mice, as described previously ${ }^{76,77}$. TAC, as a model for aortic stenosis, causes a more severe phenotype due to aortic constriction near the left ventricle. $\mathrm{AAB}$ is more moderate and reflects the disease of peripheral hypertension. Both methods were performed in a minimally invasive approach and post-surgical pain relief was performed as described for the aortocaval fistula method in the previous section.

For TAC, surgeries were done under $1.5 \%$ isoflurane insufflation. A vertical skin incision in mice aged 8-12 weeks was made at the level of the suprasternal notch, followed by dissection of muscle surrounding the trachea. The suprasternal notch was cut 4-5 $\mathrm{mm}$ into the top edge so that the aortic arch could be displayed. A 6/0-tie was passed underneath the aortic arch and then ligated around a 27-gauge needle. Afterwards, the needle was removed to allow a comparable severity of constriction. A successful surgery was visualized by enlargement of a pulsatile right carotid artery before the skin was closed. Sham animals underwent the same procedure except for banding of the aorta. This surgery was performed by Dr. Greta Sawyer (Cardiovascular Division, King's College London) and is schematically presented in Fig. 2.2. 


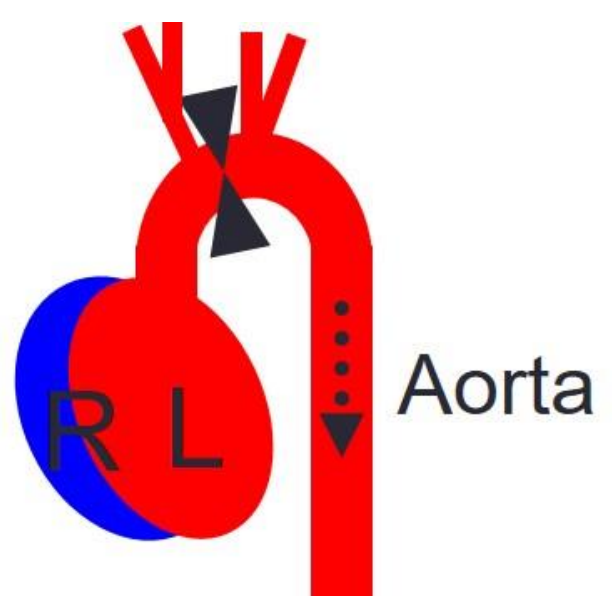

Figure 2.2: Scheme of the transverse aortic constriction (TAC) surgery. Two arrows facing each other show the location of the constriction between the brachiocephalic and left carotid artery near the left ventricle (L represent the left, $\mathrm{R}$ the right ventricle). The dotted arrow shows the direction of the blood flow in the aorta.

For $\mathrm{AAB}$, anesthesia in mice weighing $16-18 \mathrm{~g}$ was conducted by intraperitoneal injection of ketamine $(75 \mathrm{mg} / \mathrm{kg})$ plus medetomidine $\mathrm{HCl}(1 \mathrm{mg} / \mathrm{kg})$ to allow easier positioning of the animals. An incision was made below the rib cage with dissection between the coeliac and superior mesenteric arteries. An 8/0-suture was positioned around the abdominal aorta and tied against a 28-gauge needle to achieve comparable constriction. Sham surgery was performed similarly without the placement of the suture. To reverse the anesthetic effect, atipamezole $\mathrm{HCl}$ $(5 \mathrm{mg} / \mathrm{kg}$ ) was administered intraperitoneally post surgery for quick recovery. Afterwards, mice were kept in a heated chamber for three hours. This surgery was performed by Dr. Helena Zhang (Cardiovascular Division, King's College London).

\subsection{Infusion of ${ }^{13} \mathrm{C}$-labelled glucose in mice, sample preparation and ${ }^{13} \mathrm{C}$-NMR- spectroscopy}

After a six hour fasting period, mice were anesthetized under $1.5 \%$ isoflurane insufflation before an intraperitoneal bolus of $0.4 \mathrm{mg} / \mathrm{g}$ body weight $\left[\mathrm{U}^{13} \mathrm{C}\right]$ glucose (Sigma-Aldrich) was injected. $\left[\mathrm{U}_{-}{ }^{13} \mathrm{C}\right]$ is a widely used tracer for flux analysis in which all six carbons are ${ }^{13} \mathrm{C}$-labelled. The bolus injection was followed by continuous intravenous administration of $\left[\mathrm{U}-{ }^{13} \mathrm{C}\right]$ glucose with $0.012 \mathrm{mg} / \mathrm{g}$ body weight/min at $150 \mu \mathrm{L} / \mathrm{hr}$ for 30 minutes as this was the optimal time point for maximum glucose enrichment in the heart. Afterwards, the heart was rapidly snap-frozen by freeze-clamping to stop any enzymatic reactions. 
Heart tissue was minced and homogenized in an extraction buffer containing 1:1:1 ratios of methanol, chloroform and water. $1.7 \mathrm{~mL}$ buffer were added per $0.2 \mathrm{~g}$ of tissue sample and homogenized using a Precellys homogenizer at $5000 \mathrm{rpm}$ twice for 20 seconds each. The extract was then vortexed for 15 minutes before centrifugation at $1500 \mathrm{rpm}$ at $4^{0} \mathrm{C}$ for 30 minutes. The supernatant was collected, dried and finally reconstituted in $170 \mu \mathrm{L}$ of $100 \%$ deuterium oxide containing 0.5 mM of DSS (4,4-dimethyl-4-silapentane-1-sulfonic acid; Sigma Aldrich), $100 \mathrm{mM}$ sodium phosphate (pH 7.0) and 6 mM imidazole (Sigma Aldrich).

The following NMR (nuclear magnetic resonance) experiments were performed with a Bruker Avance $700 \mathrm{MHz}$ spectrometer. This method is based on the principle that all nuclei have nuclear spin with certain directions. These directions are associated with different energy states. The energy of a distinct frequency required to induce changes in spin direction is called resonance frequency and depends on the type of nuclei (for example ${ }^{1} \mathrm{H}$ or ${ }^{13} \mathrm{C}$ ). In a biological sample, this resonance frequency changes depending on which compound the nuclei are part of. This change, when compared to a reference compound, is termed chemical shift and is measured in parts per million (ppm). Thus, components of a biological sample can be identified according to their characteristic chemical shifts. The amount of these compounds present in the sample can be estimated by the peak intensity of the energy absorbed (area of NMR signal). In this study, ${ }^{13} \mathrm{C}$ isotopomer information was extracted from the highly sensitive HSQC (Heteronuclear Single Quantum Coherence) spectrum. This is a two dimensional spectrum for ${ }^{13} \mathrm{C}$ and attached ${ }^{1} \mathrm{H}$ giving one intensity peak per pair of coupled nuclei. Tracing of $\left[\mathrm{U}-{ }^{13} \mathrm{C}\right]$ glucose flux using this method was ideal for assessing cardiac glucose metabolism in pressure versus volume overload. The following readouts were used for different pathways involving glucose (Chapter 4): [1,2,3$\left.{ }^{13} \mathrm{C}\right]$ lactate for glycolysis, $\left[2,3-{ }^{13} \mathrm{C}\right]$ glutamate for anaplerosis, $\left[4,5-{ }^{13} \mathrm{C}\right]$ glutamate for $\mathrm{PDH}$ activity as suggestive marker for mitochondrial oxidative metabolism and $\left[4,5-{ }^{13} \mathrm{C}\right]$ glutamine for glutamine synthesis. This method was performed by Dr. Mei Chong (Cardiovascular Division, King's College London).

\subsection{Organ harvesting}

For organ harvesting, anesthesia in mice was induced in a chamber containing 5\% isoflurane and maintained with $2 \%$ isoflurane insufflation. The ribcage was opened to expose the beating heart, followed by intracardiac injection of $200 \mu \mathrm{L} \mathrm{5 \%}$ potassium chloride $(\mathrm{KCl})$ to arrest the heart in diastole. Firstly, the two atria were removed, washed in ice-cold phosphate-buffered saline 
(PBS), dried and weighed using a fine balance (Sartorius, Germany). The remaining heart was then excised at the level of the great vessels, followed by separation of the right and left ventricle. Both ventricles were washed in PBS, dried and weighed. The left ventricle was subsequently cut into three pieces: the apical piece was used for RNA isolation, the middle section for histology and the base for protein isolation and glutathione measurements. The apex and basal parts were frozen in liquid nitrogen and kept at $-80^{\circ} \mathrm{C}$, the middle part was stored in $4 \%$ paraformaldehyde (PFA) for two to four hours at room temperature. After fixing in PFA, each sample was washed in PBS and stored at $4^{\circ} \mathrm{C}$ in $70 \%$ ethanol until ready for paraffin embedding.

Lung, liver and kidneys were also removed, washed in PBS and dried before weighing. The tibial length was used as parameter to normalize organ weights. Both tibias were isolated by separating the distal leg at the tibiofemoral and tibiotalar joints. Surrounding mucle, ligaments and cartilage were removed and the length was measured using a millimetre scale calliper (accurate to 0.1 $\mathrm{mm})$.

\subsection{Assessment of kidney function in mice}

Murine kidney function in vivo was assessed by acute diuretic stress tests. Mice were injected intraperitoneally with $20 \mathrm{~mL} / \mathrm{kg}$ bodyweight of $0.9 \%$ saline w/v (Aqupharm). They were subsequently placed into a clean, individual metabolic chamber (Tecniplast 3600M021) without access to food or water. The urine produced by each animal was collected for four hours before its total volume was measured. This was compared to the volume of saline injected and a ratio of volume intake versus urine volume produced was calculated.

In addition, levels of urea were measured in murine blood plasma samples. This was conducted using the QuantiChrom ${ }^{\text {TM }}$ Urea Assay (BioAssay Systems), which utilizes a chromogenic reagent to form complexes specifically with urea. The intensity of the colour measured at $520 \mathrm{~nm}$ was directly proportional to the urea concentration in the sample. Briefly, blood from anesthetized mice was withdrawn via cardiac puncture and immediately stored on ice in tubes containing heparin to prevent clotting. Blood samples were centrifuged at $4^{\circ} \mathrm{C}$ with $3000 \mathrm{rpm}$ for ten minutes to separate the plasma fraction. Light intensity at $520 \mathrm{~nm}$ was measured in blood plasma samples after incubation with the chromogenic reagent. Conducting additional measurements with water and a known urea standard $(50 \mathrm{mg} / \mathrm{dL})$ allowed quantification of estimated urea concentrations in the plasma samples. 


\subsection{Echocardiography}

Anesthesia in mice was induced in an induction chamber under $5 \%$ isoflurane and $2 \mathrm{~L} / \mathrm{min}_{2}$ for 60 seconds, and maintained with $1.5 \%$ isoflurane insufflation. Heart rate and respiratory rate were kept consistent between experimental groups (400-510 bpm, 120-180 RR) using limb electrodes. The body temperature was monitored using a rectal probe and maintained at $37^{\circ} \mathrm{C}$. Transthoracic echocardiography was performed using a Vevo 2100 Imaging System with a 40 MHz linear probe (Visualsonics, Canada). Standard parameters such as left ventricular dimension (left ventricular end-diastolic/end-systolic diameters and volumes), hypertrophy (septum and posterior wall thickness) and systolic function (ejection fraction, fractional shortening, stroke volume) were assessed using anatomical M-Mode in long- and short-axis views. The relative wall thickness as a marker for concentric versus eccentric remodelling was calculated as follows: (Septum thickness + posterior wall thickness) / left ventricular end-diastolic diameter. An increase in relative wall thickness indicates concentric, and a decrease eccentric remodelling. Advanced echocardiographic assessment of cardiac function and physiology, as presented in Chapter 3, was performed using the following techniques:

Transmitral Doppler profiles were acquired using a pulsed wave Doppler in the apical 4-chamber view. The sample volume was placed close to the tip of the mitral leaflets in the mitral orifice parallel to the blood flow in order to record maximal transmitral flow velocities ${ }^{78}$.

Tissue Doppler imaging in the apical 4-chamber view was performed placing the pulsed wave Doppler close to the anterior mitral annulus to record the velocity of changes of myocardial structures.

Apical 4-chamber view was used to measure the area of the left atrium. This measurement was consistently performed after closure of the mitral valve in early systole.

Speckle tracking echocardiography was performed as described recently by our group ${ }^{79}$. Using the Vevo2100 Imaging Software 1.5.0, a number of tracking points were placed on the endocardial and epicardial border in parasternal long-axis views. Subsequent frame by frame tracking throughout the cardiac cycle allowed automatic calculation of ejection fraction (EF) as well as strain and strain rates. The software automatically divided the left ventricle into six segments and both radial and longitudinal strain and strain rate values were measured separately for each segment. The strain data presented in this study are the averages from these six different values per heart. Using the "reverse peak" option (Fig. 2.3A) during imaging analysis, the peak 
longitudinal strain rate during early filling could automatically be determined (Fig. 2.3B) for all segments. This was an additional parameter to specifically assess diastolic properties in hearts following pressure and volume overload (Chapter 3).

A

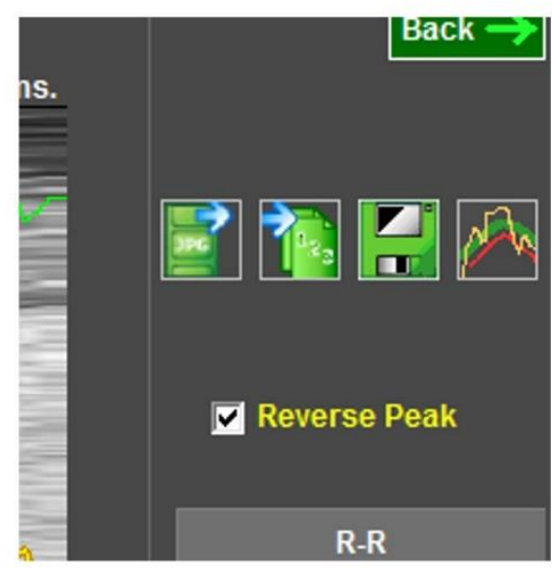

B

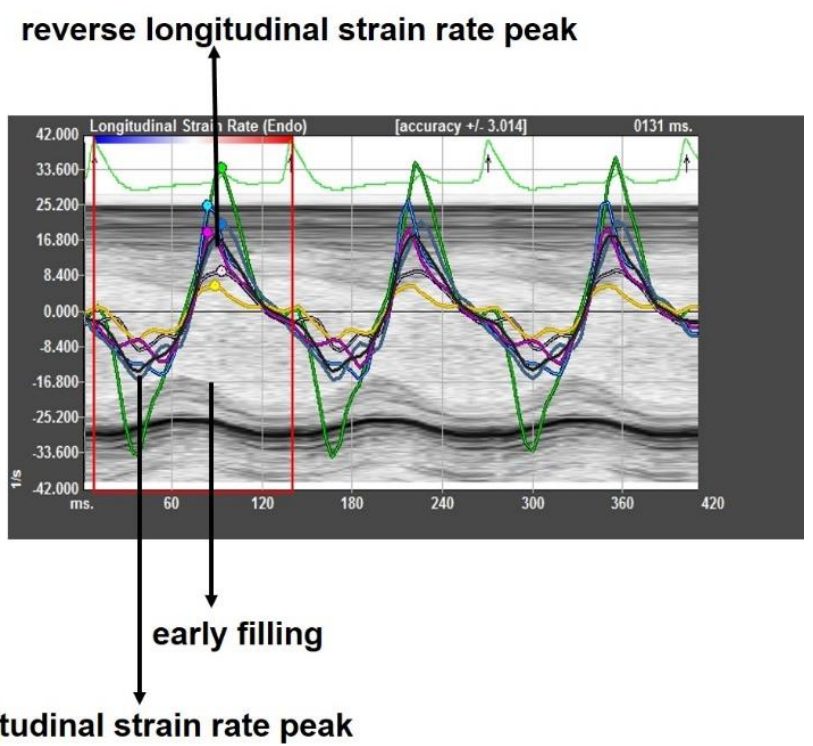

Figure 2.3: Technical and physiological aspects of assessing the reverse longitudinal strain rate in murine echocardiography. The Vevo2100 1.5.0 Image Software (Visualsonics, Canada) allows to automatically measure reverse strains and strain rates by ticking the "reverse peak" box, which is exemplarily depicted in $\mathbf{A}$. B shows a typical longitudinal strain rate profile of a WT mouse heart at baseline using speckle tracking analysis. Different colours represent different left ventricular segments. Both the longitudinal strain rate and the reverse longitudinal rate peaks are emphasized. The reverse longitudinal strain rate peak correlates time-wise with the early filling phase of the ventricle, which is visualized in the respective M-Mode image.

\subsection{RNA isolation, cDNA synthesis and quantitative real-time polymerase chain reaction (qRT-PCR)}

Tissue samples were lysed using ceramic beads (Lysing Matrix D from MP Biomedicals) and the FastPrep-24 homogenizer (MP Biomedicals). The lysates were further processed using SV total RNA Isolation Systems (Promega) according to the manufacturer's instructions. Total RNA was eluted in $50 \mu \mathrm{l}$ nuclease-free water and the concentration was measured using a NanoDrop spectrophotometer (ND-1000, Labtech International). The RNA was stored at $-80^{\circ} \mathrm{C}$. 
cDNA was synthesized by reverse transcription (RT) as follows: $1 \mu \mathrm{g}$ RNA of each sample was diluted with nuclease-free water to a total volume of $17.5 \mu 1$. $1 \mu \mathrm{l}$ of an Oligo(dT)18-primersolution $(1 \mu \mathrm{g} / \mu \mathrm{l})$ and $1 \mu \mathrm{l}$ dNTPs $(10 \mathrm{mM})$ were added to each sample before they were heated for 3 minutes at $70^{\circ} \mathrm{C}$. Immediately afterwards they were kept on ice. Then $5 \mu \mathrm{l}$ of a $5 \mathrm{X}$ RT buffer (Promega) and 0.5 $\mu \mathrm{l}$ M-MLV (Moloney murine leukaemia virus, Promega) reverse transcriptase were added to reach a volume of $25 \mu 1$. This was incubated at $42^{\circ} \mathrm{C}$ for 90 minutes. Final heating up to $70^{\circ} \mathrm{C}$ for ten minutes stopped the RT reaction and $75 \mu$ of nuclease-free water were added to each sample to reach a final volume of $100 \mu 1$.

Relative gene expression was assessed via qRT-PCR. Thus, transcripts of interests were amplified and measured using SYBR green fluorescent dye. Each reaction contained the following components: $10 \mu \mathrm{l}$ of $2 \mathrm{X}$ SYBR green master mix (PCR Biosystems), $2 \mu \mathrm{l}$ of a primer mix (9 $\mu \mathrm{M}$ of both forward and reverse primers), $2 \mu 1$ of template cDNA and $6 \mu 1$ of nuclease-free water to reach a final volume of $20 \mu \mathrm{l}$ for each reaction. Samples were amplified using a StepOnePlus ${ }^{\mathrm{TM}}$ Real-Time PCR system (Applied Biosystems). This included initial denaturation at $90^{\circ} \mathrm{C}$ for ten minutes, followed by 40 cycles of: $95^{\circ} \mathrm{C}$ for 15 seconds and $60^{\circ} \mathrm{C}$ for 30 seconds. Relative mRNA expression was calculated using the $\Delta \Delta \mathrm{Ct}$ method with GAPDH (glyceraldehyde-3-phosphate dehydrogenase) as denominator. Specific primers used in this study were designed using the NCBI primer design tool and can be found in Tables 2.3 and 2.4 respectively. Quality and specificity of primers was confirmed by detection of one single peak in the melt curve after product amplification. 
2. Material and Methods

\begin{tabular}{|c|c|c|}
\hline Primer Set & Forward $\left(5^{\prime}-3^{\prime}\right)$ & Reverse $\left(5^{\prime}-3^{\prime}\right)$ \\
\hline Acaca & GGAGATGTACGCTGACCGAG & TACCCGACGCATGGTTTTCA \\
\hline Acacb & TGAATCTCACGCGCCTACTA & TTGTGTTCTCGGCCTCTCTT \\
\hline Cpt1b & TGCCTTTACATCGTCTCCAA & AGACCCCGTAGCCATCATC \\
\hline Cpt2 & CACCTGCTCGCTCAGGATAA & CGACAGAGTCTCGAGCAGTT \\
\hline Fasn & CAAGCAGGCACACACAATGG & GTTCGTTCCTCGGAGTGAGG \\
\hline Gapdh & ATGACAACTTTGTCAAGCTCATTT & GGTCCACCACCCTGTTGCT \\
\hline$H k 1$ & GTGGACGGGACGCTCTAC & TTCACTGTTTGGTGCATGATT \\
\hline$H k 2$ & CAACTCCGGATGGGACAG & CACACGGAAGTTGGTTCCTC \\
\hline Ldha & GGCACTGACGCAGACAAG & AGCTTGATCACCTCGTAGGC \\
\hline Pdha1 & GTAAGGGGCCCATCCTGA & TCTTCTCGAGTGCGGTAGC \\
\hline Pdk1 & TTACGGATTGCCCATATCACGCCT & TCAGCCTCGTGGTTGGCTTTGTAA \\
\hline Pdk4 & CGCTTAGTGAACACTCCTTCG & CTTCTGGGCTCTTCTCATGG \\
\hline Slc2a1 (Glut1) & ATGGATCCCAGCAGCAAG & CCAGTGTTATAGCCGAACTGC \\
\hline
\end{tabular}

Table 2.3: Primer sequences for metabolic enzymes (Chapter 4).

\begin{tabular}{|c|c|c|}
\hline Primer Set & Forward $\left(5^{\prime}-3^{\prime}\right)$ & Reverse $\left(5^{\prime}-3^{\prime}\right)$ \\
\hline Acta1 ( $\alpha$-skeletal actin) & ATGCTTCTAGGCGCACTCGCGT & CACGTCAAAAACAGGCGCCGG \\
\hline Atp2a2 (Serca-2 $\alpha$ ) & GTCTCCACATTTCTCTGCAAAATG & TAGAGCAATCTGGCCACTTACAAC \\
\hline Cyba (p22phox) & TGCCCTCCACTTCCTGTT & GCAGATAGATCACACTGGCAAT \\
\hline Cybb (Nox2) & ACTCCTTGGGTCAGCACTGG & GTTCCTGTCCAGTTGTCTTCG \\
\hline Gapdh & ATGACAACTTTGTCAAGCTCATTT & GGTCCACCACCCTGTTGCT \\
\hline Gclc & GTTATGGCTTTGAGTGCTGCAT & ATCACTCCCCAGCGACAATC \\
\hline Gsta2 & GCTTGATGCCAGCCTTCTG & GGCTGCTGATTCTGCTCTTGA \\
\hline Ncf1 (p47phox) & GGACACCTTCATTCGCCATA & CTGCCACTTAACCAGGAACAT \\
\hline Ncf2 (p67phox) & TTGAACCTGTCACACAGCAAT & CCAGCACACACACAAACCTT \\
\hline Ncf4 (p40phox) & CTGCTTTTCTGACTACCCACAG & AAGCTGCTCAAAGTCGCTCT \\
\hline Nox4 & CCGGACAGTCCTGGCTTATC & TGCTTTTATCCAACAATCTTCT \\
\hline Nppa (ANP) & CGTGCCCCGACCCACGCCAGCATGGGCTCC & GGCTCCGAGGGCCAGCGAGCAGAGCCCTCA \\
\hline Nppb (BNP) & AAGGGAGAATACGGCATCATTG & ACAGCACCTTCAGGAGATCCA \\
\hline Txnrd1 & GATGCACCAGGCAGCTTTTG & TCTTCGACTTTCCAGCCATAGT \\
\hline
\end{tabular}

Table 2.4: Primer sets for genes involved in cardiac stress and redox signalling (Chapters 3 and 5). 


\subsection{Protein isolation and Western Blotting}

Using ceramic beads and the Fastprep-24 homogenizer (MP Biomedicals), snap-frozen heart tissue samples were lysed and homogenized for protein extraction in a buffer containing $25 \mathrm{mM}$ Tris-HCl, $150 \mathrm{mM} \mathrm{NaCl}, 2 \mathrm{mM}$ EGTA, $5 \mathrm{mM}$ EDTA, 0.5\% NP-40 and protease/phosphataseinhibitor cocktails (1:100 v/v; Sigma-Aldrich). Specific membrane fractions from these lysates could be obtained through additional centrifugation steps: first at 18,000 g for 15 minutes to remove mitochondria and nuclei, followed by $100,000 \mathrm{~g}$ for 45 minutes in an ultracentrifuge (Optima $^{\mathrm{TM}}$

MAX Ultracentrifuge, Beckman Coulter, USA) to get rid of the cytosolic fraction. The remaining pellet contained the membrane-enriched fraction. Protein concentrations in all homogenates were measured using Bradford reagent ${ }^{80}$. Briefly, this reagent contains Coomassie Brilliant Blue G250, which changes its absorption spectrum maximum from 465 to $595 \mathrm{~nm}$ when bound to proteins. This change in absorption could be detected spectroscopically and compared to a standard curve of known BSA (bovine serum albumin) concentrations. This allowed calculations of absolute protein concentration in the lysates. After brief sonication, the appropriate volume of 5X Laemmli buffer (300 mM Tris- $\mathrm{HCl} \mathrm{pH} 6.8,10 \%$ SDS w/v, 50\% Glycerol w/v, 0.05\% Bromophenol blue w/v, all diluted in water; before use $20 \% \beta$-Mercaptoethanol v/v was added) was added to each sample, followed by boiling at $95^{\circ} \mathrm{C}$ for five minutes. Samples specifically used for Nox4-detection were not boiled but instead treated with $1 \mathrm{mM}$ of the reducing agent tris (2-carboxyethyl) phosphine hydrochloride (TCEP; Sigma-Aldrich). Between 25 and $40 \mu \mathrm{g}$ of protein sample were loaded into each well of a 7.5-15\% SDS-polyacrylamide gel. A 10-180 kDa protein ladder (PageRuler Prestained, Thermo Fisher) was added in one of the wells to confirm the size of detected protein bands. In the presence of a buffer containing $25 \mathrm{mM}$ Tris $\mathrm{HCl}, 200$ $\mathrm{mM}$ Glycine and $0.1 \%$ SDS v/v, electrophoresis was used to separate proteins according to their molecular weight. Afterwards, electrophoretic transfer onto nitrocellulose membranes (Hybond ECL, GE Healthcare) was performed under 100 Volts for 70 minutes in a methanol-containing buffer (25 mM Tris HCl, $200 \mathrm{mM}$ Glycine, 20\% Methanol v/v). Membranes were blocked with $10 \%$ milk in Tris-buffered saline and $0.01 \%$ Tween v/v (TBST) for two hours at room temperature under gentle agitation. This was followed by incubation with the primary antibody (diluted in either $5 \%$ milk w/v or $5 \% \mathrm{BSA} w / \mathrm{v}$ in TBST) overnight at $4^{\circ} \mathrm{C}$. Lists of primary antibodies used in this study can be found in Tables 2.5 and 2.6, specific Nox4-antibodies were produced by our group ${ }^{81}$. 
After incubation with the primary antibody, membranes were washed three times with TBST for ten minutes each, followed by incubation with Li-Cor secondary antibodies-conjugated to fluorophores (IRDye 680RD and 800RD anti-mouse IgG or anti-rabbit IgG; diluted 1:15000 in $5 \%$ milk w/v in TBST) for one hour at room temperature. After three more TBST washes for ten minutes each, images were acquired using the Odyssey® CLx imager (Li-Cor). Image analysis and densitometric quantification of specific protein bands were performed with Image Studio 2.1. GAPDH was used as loading control for whole heart lysates, caveolin-3 for membrane-enriched fractions.

\begin{tabular}{|c|c|}
\hline Primary Antibody & Dilution, Company \\
\hline$\alpha$-Tubulin & $1: 10000$ in $5 \%$ milk, Sigma-Aldrich \\
\hline$\beta$-Actin & $1: 10000$ in $5 \%$ milk, Sigma-Aldrich \\
\hline Glucose transporter 1 (GLUT1) & $1: 2000$ in $5 \%$ milk, Abcam \\
\hline Glutaminase (GLS) & $1: 2000$ in $5 \%$ milk, Abcam \\
\hline Glutamine synthetase (GS) & $1: 2000$ in $5 \%$ milk, Abcam \\
\hline Glutamine fructose-6-phosphate amidotransferase 1 (GFAT1) & 1:2000 in $5 \%$ milk, Proteintech \\
\hline Glutamine fructose-6-phosphate amidotransferase 2 (GFAT2) & $1: 2000$ in $5 \%$ milk, Proteintech \\
\hline Glyceraldehyde 3-phosphate-dehydrogenase (GAPDH) & 1:10000 in 5\% milk, Sigma-Aldrich \\
\hline Hexokinase 1 (HK1) & $1: 2000$ in $5 \%$ milk, Abcam \\
\hline Lactate dehydrogenase A (LDHA) & $1: 2000$ in $5 \%$ milk, Proteintech \\
\hline O-linked N-Acetylglucosamine (GIcNAc) & $1: 2000$ in $5 \%$ milk, Abcam \\
\hline Pyruvate carboxylase (PC) & $1: 2000$ in $5 \%$ milk, Santa Cruz \\
\hline Pyruvate dehydrogenase e1 $\alpha$ (PDHe1 $\alpha)$ & $1: 2000$ in $5 \%$ milk, Abcam \\
\hline Pyruvate dehydrogenase kinase 1 (PDK1) & $1: 2000$ in $5 \%$ milk, Abcam \\
\hline Pyruvate dehydrogenase kinase 4 (PDK4) & $1: 2000$ in $5 \%$ milk, Abcam \\
\hline
\end{tabular}

Table 2.5: Antibodies detecting proteins involved in cardiac metabolism (Chapter 4). 


\begin{tabular}{|l|c|}
\hline \multicolumn{1}{|c|}{ Primary Antibody } & Dilution, Company \\
\hline Caveolin-3 & $1: 5000$ in 5\% milk, Sigma-Aldrich \\
\hline elF4E-BP1 & $1: 1000$ in 5\% milk, Abcam \\
\hline Glyceraldehyde 3-phosphate dehydrogenase (GAPDH) & $1: 10000$ in 5\% milk, Sigma-Aldrich \\
\hline gp91 ${ }^{\text {phox }}$ (Nox2) & $1: 1000$ in 5\% milk, BD Biosciences \\
\hline Nox4 & $1: 1000$ in $5 \%$ milk, produced in our lab \\
\hline Phospho-Akt (S473) & $1: 1000$ in 5\% BSA, Cell Signaling \\
\hline Phospho-Erk1/2 (Thr202/Tyr204) & $1: 1000$ in 5\% BSA, Cell Signaling \\
\hline Phospho-S6 ribosomal protein (S235/236) & $1: 1000$ in 5\% BSA, Cell Signaling \\
\hline p47phox & $1: 1000$ in 5\% milk, EMD Millipore \\
\hline Total Akt & $1: 1000$ in 5\% milk, Cell Signaling \\
\hline Total Erk1/2 & $1: 1000$ in 5\% milk, Cell Signaling \\
\hline Total S6 ribosomal protein & $1: 1000$ in 5\% milk, Cell Signaling \\
\hline
\end{tabular}

Table 2.6: Antibodies detecting proteins involved in cardiac hypertrophy and redox signalling (Chapter 5).

\subsection{Histology}

Using a microtome (Leica), paraffin-embedded heart tissues were sectioned with a thickness of 6 $\mu \mathrm{m}$ each and placed on a glass slide. Sections were then dewaxed by two separate ten minute incubations in xylene, followed by graded rehydration: starting in 100\% ethanol, then $96 \%, 80 \%$, $70 \%, 50 \%, 25 \%$, ending in water (five minutes for each incubation). Depending on the protocols for different staining procedures, sections were processed as follows:

\section{$\underline{\text { 2.11.1 Apoptosis }}$}

Apoptosis was assessed by indirect TUNEL (TdT-mediated dUTP-biotin nick end labeling)staining with the ApopTag® Red In Situ Apoptosis Detection Kit (Merck Millipore S7165) according to the manufacturer's instructions. Briefly, terminal deoxynucleotidyl transferase (TdT) catalyzes the addition of labelled and unlabelled nucleotide triphosphates to 3'OH-DNA termini of apoptotic cells. These nucleotides form an oligomer with digoxigenin. Utilizing an anti-digoxigenin antibody with a rhodamine fluorochrome, apoptotic cells could be detected using the Olympus IX-81 microscope. 4',6-Diamidino-2-phenylindole (DAPI; $0.5 \mu \mathrm{g} / \mathrm{ml}$ ) was used to stain all nuclei. Image analysis included quantification of apoptotic versus total cells and was performed with the Fiji software (ImageJ). 


\subsubsection{Fibrosis}

Sections were immersed in $0.2 \%$ phosphomolybdic acid w/v (diluted in water; Sigma-Aldrich), for two minutes. Then, they were washed in water three times and immersed in $0.1 \%$ Picrosirius red solution w/v (diluted in saturated picric acid; Sigma-Aldrich) for two hours. Slides were removed and washed in two changes of acidified water $(0.5 \%$ acetic acid v/v) for two minutes, followed by graded dehydration in increasing ethanol concentrations (starting from 25\%, 50\%, $75 \%$ and then $100 \%$, incubation for one minute each). Sections were immersed in xylene for one minute before finally mounting the slides with DPX mounting medium and placement of the coverslip. Images were acquired using the Olympus IX-81 microscope. The proportion of fibrosis was calculated by the area of red staining relative to the total area of the heart tissue slice using Volocity software (Perkin Elmer).

\section{$\underline{\text { 2.11.3 Cardiomyocyte area and capillary density }}$}

Staining for cardiomyocyte area with WGA (wheat germ agglutinin)-rhodamine and capillary density using Isolectin B4 could be conducted in parallel on the same sections. Slides were immersed in $1 \%$ of a Tris-based antigen unmasking solution v/v (diluted in water; Vectorlabs) under steaming conditions for ten minutes, followed by incubation with the steamer switched off and finally with the steamer-lid taken off for ten minutes each. Slides were washed with PBS three times for five minutes each before blocking nonspecific protein binding with $10 \%$ goat serum v/v (diluted in PBS; Vectorlabs) for one hour in a humidified chamber at room temperature. Excess goat serum was removed and Isolectin B4 (1:150 v/v diluted in 10\% goat serum; Vectorlabs) was added onto the sections and left overnight in a humidified chamber at $4^{\circ} \mathrm{C}$. On the following day, slides were washed with PBS three times for five minutes each. This was followed by simultaneous incubation with $1 \%$ avidin-FITC v/v (Vectorlabs) and 2\% WGArhodamine v/v (Vectorlabs), diluted together in 10\% goat serum, for two hours in a humidified chamber at room temperature. Finally, sections were washed with PBS and mounted with mowiol before placement of a coverslip. Images were acquired with the Olympus IX-81 microscope, image analysis was performed with Fiji (ImageJ).

\subsection{Glutathione measurements}

Glutathione is one of the main intracellular redox buffers. The ratio of reduced versus oxidized glutathione gives an indication of the general redox status in biological samples. In this study, measurements were performed using the GSH-Glo ${ }^{\mathrm{TM}}$ Assay kit according to the manufacturer's 
protocol (Promega). In the presence of reduced glutathione (GSH), glutathione S-transferase (GST) catalyzed the conversion of a luciferin-derivate to luciferin, which could be detected through a coupled reaction generating luminescence. The amount of GSH was proportional to the luminescence intensity. Briefly, snap-frozen heart tissue was homogenized in phosphate-buffered saline containing $2 \mathrm{mM}$ EDTA to reach a concentration of $20 \mathrm{mg}$ tissue/ml buffer. Levels of GSH could be measured directly in the lysate as previously described, total glutathione (GSH+GS-SG) was assessed after pre-incubation of the lysates with the reducing agent TCEP (Sigma-Aldrich) in a concentration of $1 \mathrm{mM}$ before continuing with the assay. Oxidized glutathione levels (GS-SG) as well as the ratio between reduced versus oxidized glutathione (GSH/GS-SG) were calculated afterwards.

\subsection{Statistical analysis}

Statistical analyses (including graphical presentations) were performed using GraphPad Prism version 6. Data are shown as the mean \pm SEM. Differences in means were compared by either an unpaired Student's t-test (Chapters 3 and 4) or two-way ANOVA followed by Bonferroni posthoc test for multiple comparisons (Chapter 5). Correlations were assessed using linear regression and coefficients of determination $\left(\mathrm{r}^{2}\right)$. Differences between groups were considered significant if the p-value was $<0.05$. 


\section{Results I: Non-invasive assessment of cardiac physiology in murine models of volume and pressure overload}

\subsection{Introduction}

Pressure and volume overload are common cardiac stressors in various cardiovascular diseases. Amongst these are valvular heart diseases with a reported prevalence of $2.5 \%$ in the US population, according to a study from $2006^{82}$. In order to better understand the molecular pathogenesis and pathophysiology of these diseases, studying mice has become an invaluable tool in cardiovascular research due to their homogeneity and easy maintenance. Additionally, their genome can be altered easily and multiple procedures have been established to model human diseases. The transverse aortic constriction (TAC) model is used to mimic severe pressure overload in the heart, as seen in aortic stenosis. Abdominal aortic banding (AAB) causes a more moderate increase in pressure overload and is therefore used to model chronic arterial hypertension. Despite its clinical relevance, studies focusing on volume overload models, especially in mice, can hardly be found throughout the literature. A disease typically associated with volume overload is aortic regurgitation, which is caused by incomplete closure of the aortic valve during diastole. More than $2 \%$ of people aged 70 years or older suffer from at least moderate or even severe aortic regurgitation. The prognosis of patients with aortic regurgitation, who are symptomatic during mild activity (NYHA III) or at rest (NYHA IV), is poor with a fiveyear survival of only $28 \%{ }^{83}$. The aortocaval fistula (Shunt) is an established model to induce volume overload in mice, which has been first described 15 years ago ${ }^{75}$. This model was used in this study to characterize and assess changes in cardiac physiology during volume overload.

Diastolic heart failure, often referred to as heart failure with preserved ejection fraction (HFpEF), is associated with left ventricular diastolic dysfunction due to an impaired relaxation as well as an increase in myocardial stiffness ${ }^{84}$. This can subsequently lead to an increase in left ventricular filling pressure, left atrial pressure and pulmonary capillary pressure causing acute pulmonary edema $^{85}$. Diastolic dysfunction and its role in the development of heart failure has gained increasingly more attention over the past years. In the last two decades, the prevalence for diastolic heart failure has increased from $38 \%$ to $54 \%$ of all heart failure cases with outcomes similar to those of systolic heart failure ${ }^{86-89}$. Echocardiography remains a very important, noninvasive tool for the assessment of diastolic function in humans. The usage of techniques such as 
transmitral Doppler to assess the relaxation and early filling phase of the left ventricle (e.g. isovolumic relaxation time (IVRT), E/A (ratio of early versus late diastolic transmitral flow velocity), E-wave deceleration time (DT)), tissue Doppler (e.g. E/E', which is the ratio of early diastolic transmitral flow versus early diastolic mitral annular velocity) and apical 4-chamber view (e.g. left atrial volume) to evaluate left ventricular filling pressures are all part of a general algorithm to diagnose diastolic heart failure in humans, proposed by the European Society of Cardiology ${ }^{89}$. If active relaxation is impaired, the E/A ratio decreases due to a shift from early filling to atrial contribution for ventricular filling, whereas the IVRT and DT increase. At this point, left ventricular filling pressure is normal. When diastolic dysfunction progresses, the left atrial pressure (and left atrial size) increases and disguises the impaired relaxation, therefore leading to pseudo-normalization of these parameters. If diastolic function further deteriorates due to increased left ventricular stiffness, it reaches the so called restrictive pattern. In this case, early filling terminates abruptly due to elevated left ventricular diastolic pressures, which causes a marked decrease in IVRT and DT, as well as an increase in the E/A ratio. The E/E' as marker for left ventricular filling pressure increases as diastolic dysfunction progresses ${ }^{90-92}$.

Whereas many studies in cardiovascular research focus on the systolic function in mice using standard long- and short-axis views, the diastolic part is often neglected. One of the reasons is the technically challenging assessment of diastolic properties due to high heart rates and small organ sizes in mice. One aim of the work in this Chapter was to define the most useful parameters to assess diastolic function in mice using non-invasive echocardiography. Thus, commonly recommended techniques in human echocardiography to evaluate the diastolic function were performed in a range of different mouse models that induce chronic, haemodynamic stress (e.g. pressure overload using $\mathrm{AAB}$ and TAC, volume overload using Shunt). Myocardial strain, a normalized measure of deformation to assess regional myocardial wall motion ${ }^{93}$, was evaluated during systole and diastole in those different mouse models using speckle tracking analysis, as this technique has been gaining more interest as a sensitive parameter in both humans and mice ${ }^{79}$, 94-96. Besides evaluating different echocardiographic parameters in terms of their significance in mice, it was also aimed to get a more detailed understanding of diastolic physiology in the heart under different haemodynamic stress conditions. 


\subsection{Basic characterization of the aortocaval fistula (Shunt), a model to induce volume overload in mice}

Establishing and characterizing the aortocaval fistula model (Shunt) involved basic transthoracic echocardiography, organ weight measurements and gene expression analysis of common cardiac stress markers in mice after Shunt and their respective Sham control surgery. One and six weeks were chosen as end points to assess the progression of Shunt-induced cardiac remodelling, function and hypertrophy.

\subsubsection{Transthoracic echocardiography}

Using echocardiography, the left ventricular end-diastolic diameter (LVEDD) as parameter for eccentric remodelling was found to be significantly increased after one week (4.7 vs $4.0 \mathrm{~mm}$, $\mathrm{p}<0.01$ ), and even more pronounced after six weeks ( 5.5 vs $4.2 \mathrm{~mm}, \mathrm{p}<0.01)$ of volume overload compared to respective Sham controls (Fig. 3.1A, Table 3.1). The left ventricular end-systolic diameter (LVESD) depends on the general size, but also on the contractile function of the left ventricle. This parameter was increased significantly after six weeks of volume overload (4.1 vs $2.9 \mathrm{~mm}, \mathrm{p}<0.01)$ compared to respective Sham controls, whereas the increase after one week did not reach statistical significance (3.1 vs $2.8 \mathrm{~mm}, \mathrm{p}=0.06$ ) (Fig. 3.1B, Table 3.1). The septal wall was slightly, but significantly thicker after both one week $(0.80$ vs $0.72 \mathrm{~mm}, \mathrm{p}<0.05)$ and six weeks (0.76 vs $0.68 \mathrm{~mm}, \mathrm{p}<0.05)$ of volume overload compared to controls (Fig. 3.1C, Table 3.1). The relative wall thickness, expressed as wall thicknesses of both the septal and posterior wall divided by the LVEDD, is a parameter for concentric versus eccentric remodelling. The significant decrease after six weeks $(0.26$ vs $0.30, \mathrm{p}<0.01)$ of volume overload indicated eccentric remodelling, which was not yet evident at one week ( 0.36 vs $0.37, \mathrm{p}=\mathrm{n} . \mathrm{s}$. $)$ compared to respective controls (Fig. 3.1D, Table 3.1). 
A

LVEDD

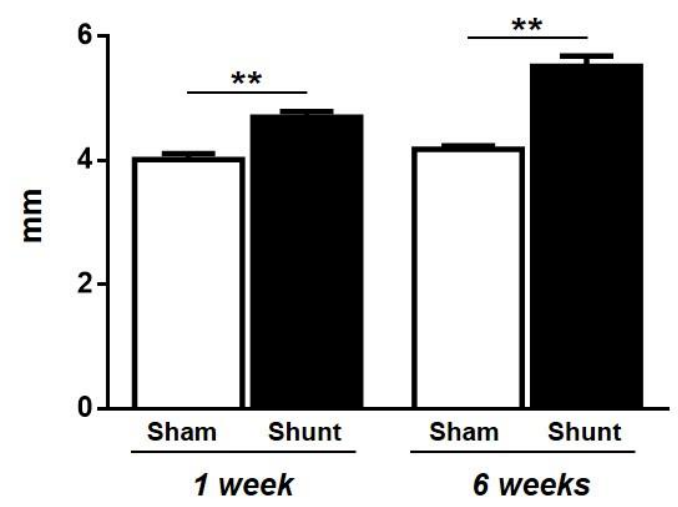

C

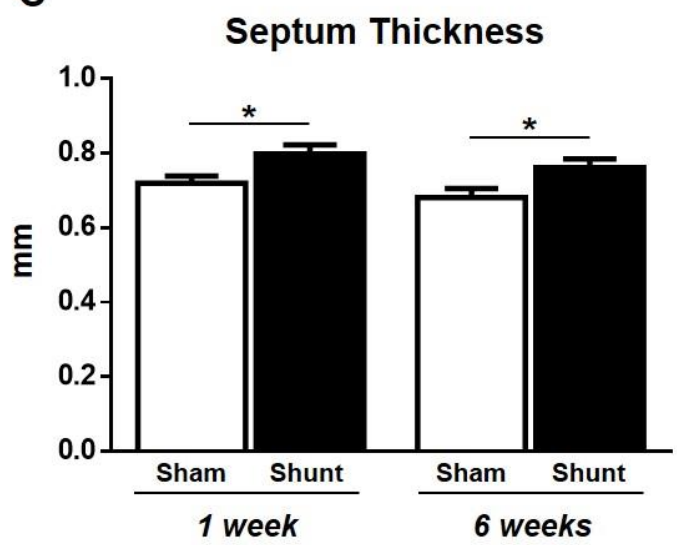

B

LVESD

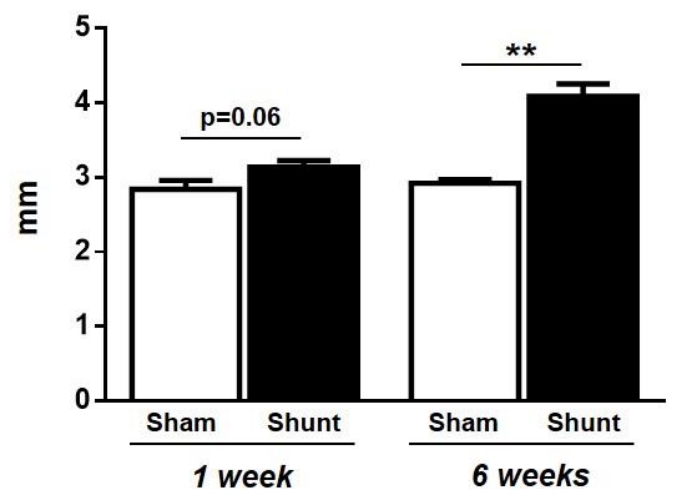

D

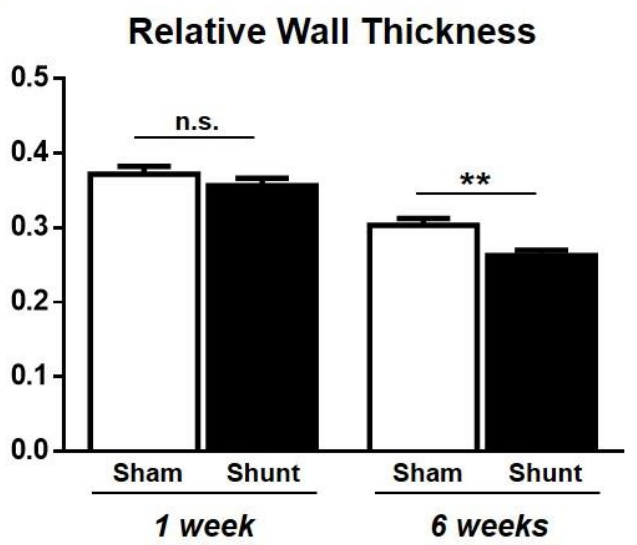

Figure 3.1: Echocardiographic assessment of cardiac remodelling in mice following volume overload. Shunt and respective Sham surgeries were performed in female WT mice. Transthoracic echocardiography was conducted one and six weeks after surgery to assess left ventricular end-diastolic diameter (LVEDD) (A), left ventricular endsystolic diameter (LVESD) (B), septum thickness (C) and the relative wall thickness (D). n=6-7/group; data are presented as mean $\pm \mathrm{SEM} ; * \mathrm{p}<0.05$, ** $\mathrm{p}<0.01$, n.s.: not significant between Shunt and respective Sham controls using unpaired Student's t-test.

The heart rate was significantly increased after one week of volume overload (475 vs $406 \mathrm{bpm}$, $\mathrm{p}<0.01$ ) in mice compared to controls as sign for an acute, hyperdynamic response. After six weeks, however, heart rates were similar (445 vs 441 bpm, p=n.s.) between groups (Fig. 3.2A, Table 3.1). Ejection fraction (EF) and fractional shortening (FS), used as parameters to assess systolic function, showed an increase after one week of volume overload (EF: 61.9 vs 56.5\%, $\mathrm{p}=0.08$; FS: 33.4 vs $29.4 \%$, p=0.07) compared to controls, indicating initial hypercontractility. After six weeks of volume overload, however, these parameters were significantly decreased (EF: 
50.5 vs $57.5 \%, \mathrm{p}<0.01$; FS: $26.0 \%$ vs $30.0 \%, \mathrm{p}<0.05)$ suggesting deterioration of systolic function under long-term volume stress (Fig. 3.2B,C, Table 3.1). Cardiac stroke volumes were significantly higher at both one week (63.6 vs $39.8 \mu 1, \mathrm{p}<0.01)$ and six weeks $(74.7$ vs $44.9 \mu 1$, $\mathrm{p}<0.01$ ) of volume overload compared to Sham controls (Fig. 3.2D, Table 3.1).

A

Heart Rate

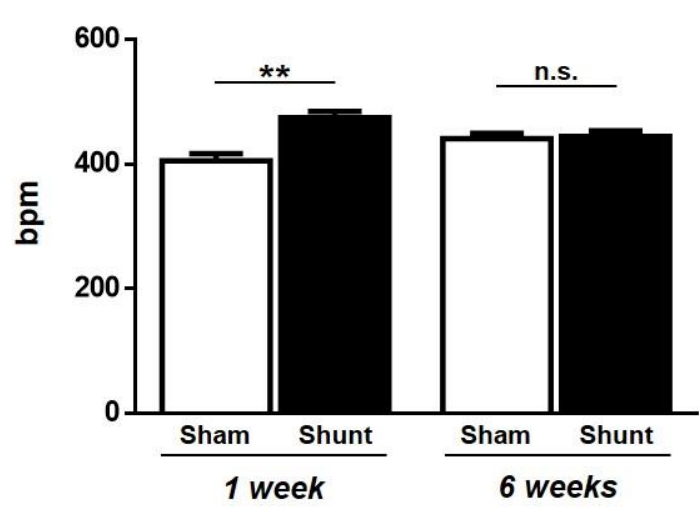

C

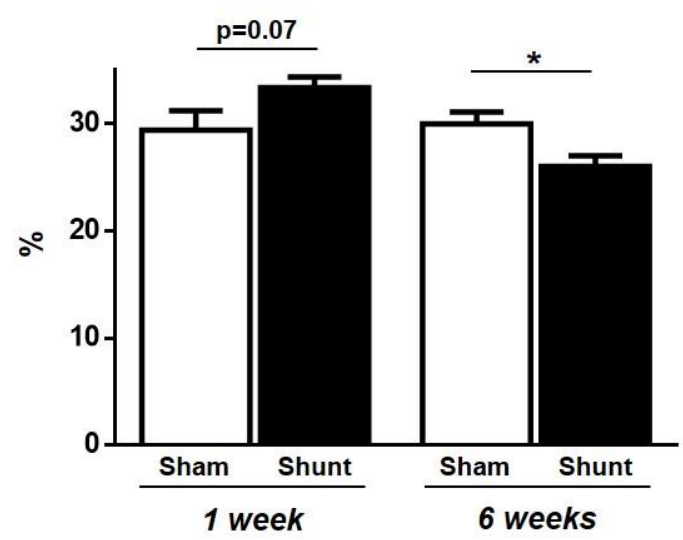

B Ejection Fraction

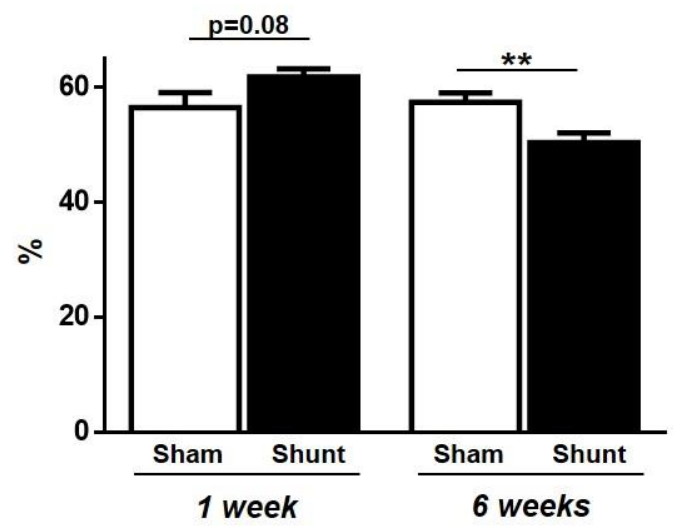

D

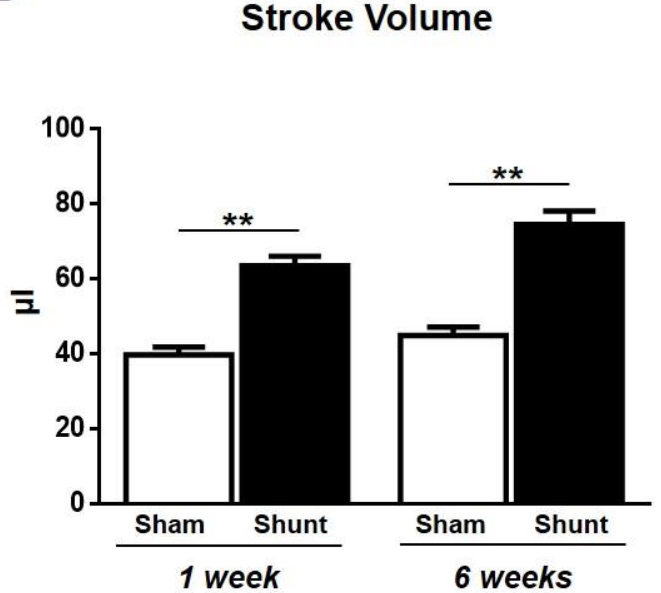

Figure 3.2: Echocardiographic assessment of cardiac function in mice following volume overload. Shunt and respective Sham surgeries were performed in female WT mice. Transthoracic echocardiography was conducted one and six weeks after surgery to assess heart rate $(\mathbf{A})$, ejection fraction $(\mathbf{B})$, fractional shortening $(\mathbf{C})$ and stroke volume (D). $\mathrm{n}=6-7$ /group; data are presented as mean $\pm \mathrm{SEM} ;{ }^{*} \mathrm{p}<0.05, * * \mathrm{p}<0.01$, n.s.: not significant between Shunt and respective Sham controls using unpaired Student's t-test. 


\begin{tabular}{|c|c|c|c|c|}
\hline & \multicolumn{2}{|c|}{1 week } & \multicolumn{2}{|c|}{6 weeks } \\
\hline & Sham & Shunt & Sham & Shunt \\
\hline$n=$ & 6 & 7 & 7 & 7 \\
\hline HR [bpm] & $406 \pm 11.5$ & $475 \pm 10.0^{* *}$ & $441 \pm 8.7$ & $445 \pm 9.0$ \\
\hline LVID;d [mm] & $4.0 \pm 0.10$ & $4.7 \pm 0.09^{* *}$ & $4.2 \pm 0.06$ & $5.5 \pm 0.17^{* *}$ \\
\hline LVID;s [mm] & $2.8 \pm 0.12$ & $3.1 \pm 0.09$ & $2.9 \pm 0.05$ & $4.1 \pm 0.17^{* *}$ \\
\hline LVV;d $[\mu l]$ & $71.0 \pm 4.0$ & $103.2 \pm 4.4^{* *}$ & $78.0 \pm 2.6$ & $150.0 \pm 10.1^{* *}$ \\
\hline LVV;s [ $\mu l]$ & $31.2 \pm 3.3$ & $39.7 \pm 2.7$ & $33.05 \pm 1.4$ & $75.0 \pm 7.3^{* *}$ \\
\hline Septum [mm] & $0.72 \pm 0.02$ & $0.80 \pm 0.02^{*}$ & $0.68 \pm 0.02$ & $0.76 \pm 0.02^{*}$ \\
\hline Post. Wall [mm] & $0.77 \pm 0.02$ & $0.85 \pm 0.03^{*}$ & $0.59 \pm 0.02$ & $0.68 \pm 0.04$ \\
\hline LV mass [mg] & $104.3 \pm 7.2$ & $154.0 \pm 8.8^{* *}$ & $92.6 \pm 5.2$ & $181.0 \pm 17.3^{* *}$ \\
\hline RWT & $0.37 \pm 0.01$ & $0.36 \pm 0.01$ & $0.30 \pm 0.01$ & $0.26 \pm 0.01^{* *}$ \\
\hline $\mathrm{SV}[\mu \mathrm{l}]$ & $39.8 \pm 2.0$ & $63.6 \pm 2.5^{* *}$ & $44.9 \pm 2.3$ & $74.7 \pm 3.5^{* *}$ \\
\hline EF [\%] & $56.5 \pm 2.6$ & $61.9 \pm 1.4$ & $57.5 \pm 1.6$ & $50.5 \pm 1.7^{* *}$ \\
\hline FS [\%] & $29.4 \pm 1.8$ & $33.4 \pm 1.0$ & $30.0 \pm 1.1$ & $26.0 \pm 0.97^{*}$ \\
\hline
\end{tabular}

Table 3.1: Echocardiographic parameters following volume overload. Transthoracic echocardiography was performed in female WT mice one and six weeks post Shunt and respective Sham controls. HR: heart rate, bpm: beats per minute, LVID;d: left ventricular diameter in diastole, LVID;s: left ventricular diameter in systole, LVV;d: left ventricular volume in diastole, LVV;s: left ventricular volume in systole, septum: septal wall thickness, post. Wall: posterior wall thickness, RWT: relative wall thickness, SV: stroke volume, EF: ejection fraction, FS: fractional shortening. Data are presented as mean \pm SEM; * $\mathrm{p}<0.05$ and ** $\mathrm{p}<0.01$ between Shunt and respective Sham controls using unpaired Student's t-test.

\subsubsection{Heart and organ weights}

Cardiac hypertrophy is a common response of the heart to many stressors including volume overload. In order to assess the amount of hypertrophy, heart weights following one and six weeks of volume overload were measured, normalized to respective tibia lengths and compared to Sham controls. Heart weight tibia length ratios increased progressively from 50\% higher after one week (9.9 vs $6.6 \mathrm{mg} / \mathrm{mm}, \mathrm{p}<0.01)$ to $64 \%$ higher after six weeks $(10.8$ vs $6.6 \mathrm{mg} / \mathrm{mm}$, $\mathrm{p}<0.01$ ) as compared to respective controls (Fig. 3.3A). To study this in more detail, all four cardiac chambers were separated and measured individually. Development of hypertrophy in 
each chamber showed a similar pattern of progression following volume overload. The left ventricular weight versus tibia length ratio increased from $40 \%$ after one week (7.2 vs 5.2 $\mathrm{mg} / \mathrm{mm}, \mathrm{p}<0.01$ ) to $53 \%$ after six weeks ( $8.0 \mathrm{vs} 5.3 \mathrm{mg} / \mathrm{mm}, \mathrm{p}<0.01$ ) (Fig. 3.3B), the right ventricular weight versus tibia length ratio from $82 \%(1.89 \mathrm{vs} 1.04 \mathrm{mg} / \mathrm{mm}, \mathrm{p}<0.01)$ to $102 \%$ (1.96 vs $0.97 \mathrm{mg} / \mathrm{mm}, \mathrm{p}<0.01$ ) (Fig. 3.3C) and the combined atrial weight versus tibia length ratio from $92 \%(0.73$ vs $0.38 \mathrm{mg} / \mathrm{mm}, \mathrm{p}<0.01)$ to $117 \%(0.76$ vs $0.35 \mathrm{mg} / \mathrm{mm}, \mathrm{p}<0.01)$ compared to respective Shams (Fig. 3.3D).

A

Heart Weight/Tibia Length

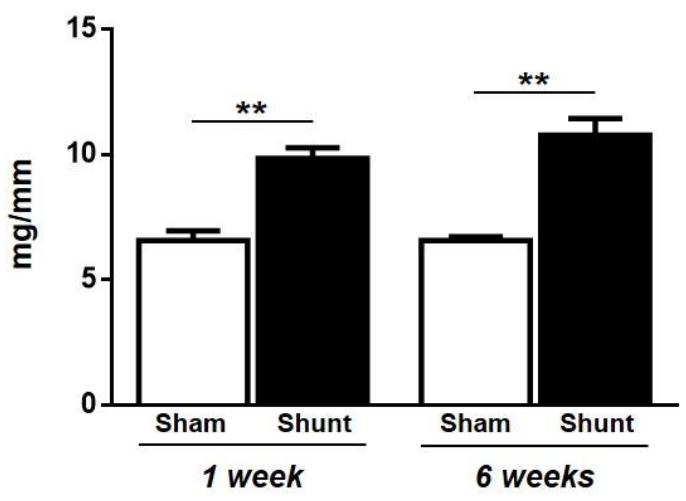

C

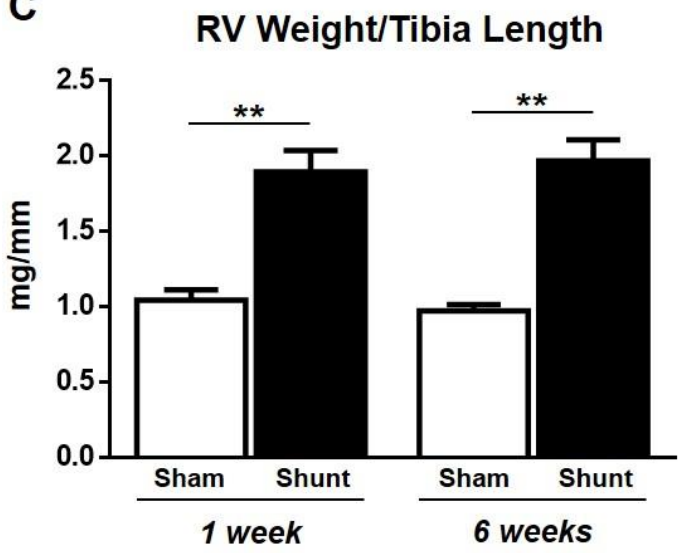

B
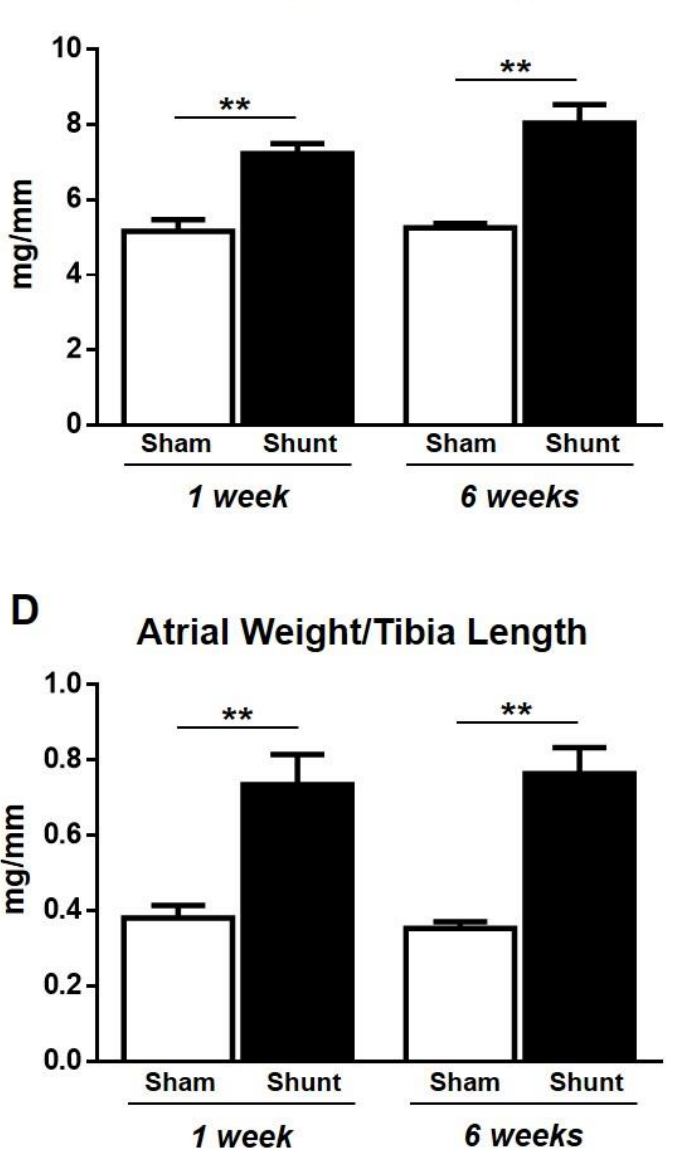

Figure 3.3: Cardiac hypertrophy following volume overload. Shunt and respective Sham surgeries were performed in female WT mice and the hearts harvested after one and six weeks respectively to measure total heart weight (A), left ventricular (LV) weight (B), right ventricular (RV) weight (C) and atrial weight (both left and right atrium) (D), all normalized to respective tibia lengths. $n=6-9$ /group; data are presented as mean \pm SEM; ** $p<0.01$ between Shunt and respective Sham controls using unpaired Student's t-test. 
Increases in body, lung, liver and kidney weights due to congestion are indicators of cardiac pump failure. To assess this, organs were harvested from mice one and six weeks following volume overload. Their dry weights were measured and normalized to respective tibia lengths. Compared to Sham controls, body weight $(25.3$ vs $23.3 \mathrm{~g}, \mathrm{p}<0.05)$ and lung weight versus tibia length ratios (9.2 vs $7.9 \mathrm{mg} / \mathrm{mm}, \mathrm{p}<0.05)$ showed only moderate increases after six weeks with unchanged liver weights. One week of volume overload caused no change of body, lung and liver weights (Fig. 3.4A-C). The average kidney weight versus tibia length ratio, however, was significantly decreased at both one week $(6.9 \mathrm{vs} 8.3 \mathrm{mg} / \mathrm{mm}, \mathrm{p}<0.05)$ and six weeks $(7.1 \mathrm{vs} 7.9$ $\mathrm{mg} / \mathrm{mm}, \mathrm{p}<0.05$ ) of volume overload compared to respective controls (Fig. 3.4D).

A

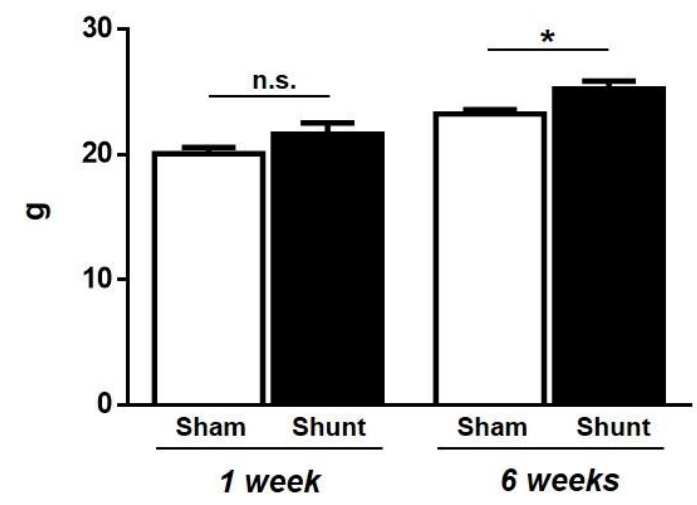

C

Liver Weight/Tibia Length

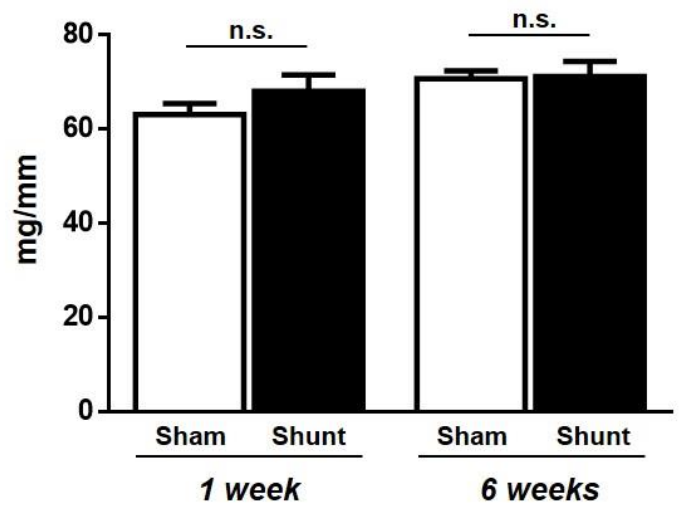

B

Lung Weight/Tibia Length

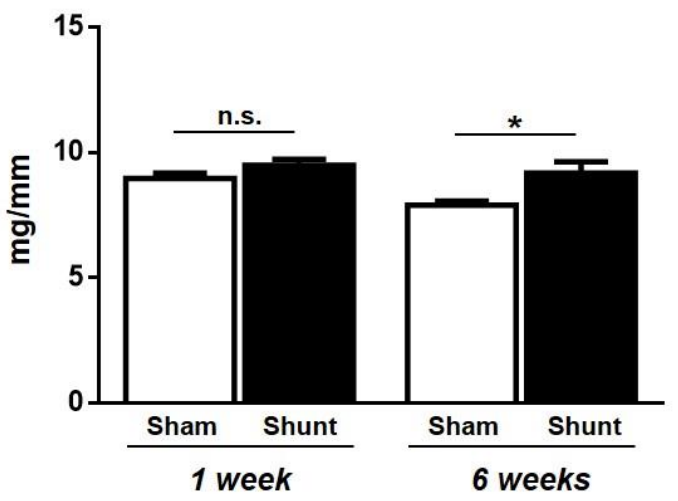

\section{D}

Average Kidney Weight/Tibia Length

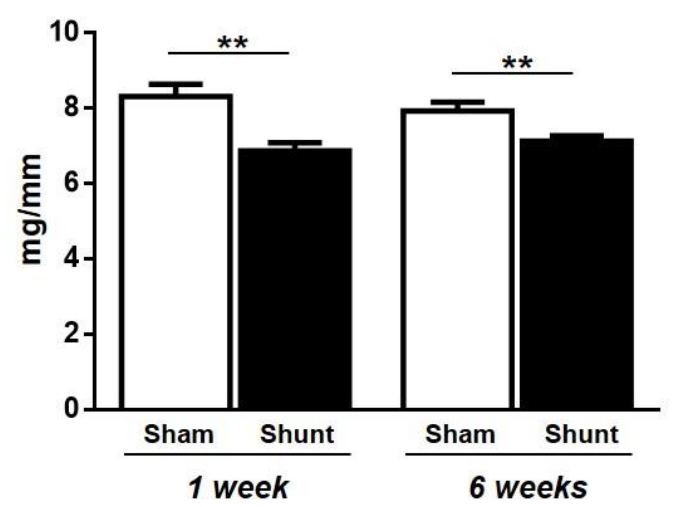

Figure 3.4: Body and organ weights following volume overload. Shunt and respective Sham surgeries were performed in female WT mice. The total body weight (A) as well as post mortem lung (B), liver (C) and average kidney weights (D) were measured after one or six weeks of volume overload and normalized to respective tibia lengths. $\mathrm{n}=6-9$ /group; data are presented as mean $\pm \mathrm{SEM} ; * \mathrm{p}<0.05$, ** $\mathrm{p}<0.01$, n.s.: not significant between Shunt and respective Sham controls using unpaired Student’s t-test. 


\subsubsection{Kidney function}

In order to assess a functional relevance of this decrease in kidney weight, acute diuretic stress tests and plasma urea levels measurements were performed in mice after six weeks of volume overload and compared to respective Sham controls. Acute diuretic stress tests in mice were performed through intraperitoneal injection of $20 \mathrm{ml} / \mathrm{kg}$ saline, followed by urine collection for four hours and subsequent volume assessment. Ratios for injected saline versus volume of urine produced were calculated and compared to respective controls. Plasma urea levels were measured using the QuantiChrom ${ }^{\mathrm{TM}}$ Urea Assay (BioAssay Systems). Despite the change in kidney weight, both readouts for kidney function following Shunt displayed similar results to Sham controls indicating no functional impairment: ratios of injected saline versus collected urine volumes was 1.09 following Shunt compared to 0.97 in controls (Fig. 3.5A), plasma urea levels were 44.7 versus $42.4 \mathrm{mg} / \mathrm{dL}$ (Fig. 3.5B). Thus, the kidney weight decrease might be attributed to a Shuntmediated perfusion deficit rather than impaired function.

A Saline injected/Urine produced

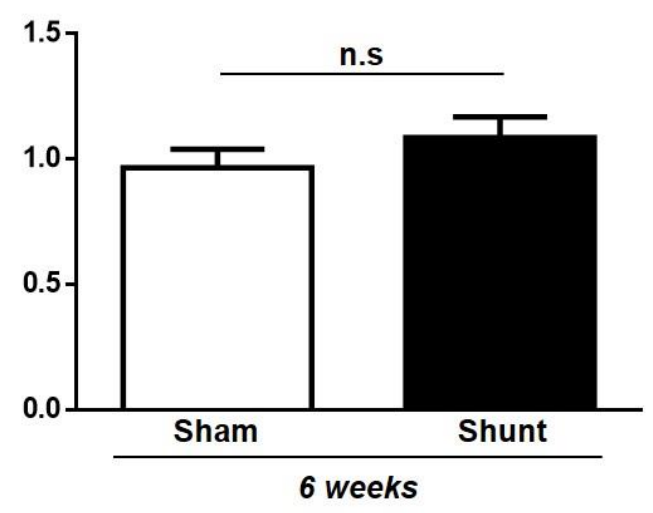

B

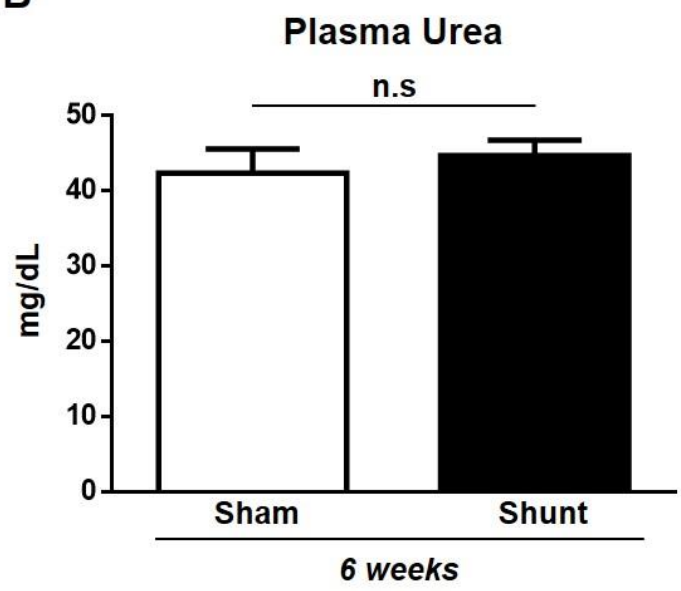

Figure 3.5: Kidney function following volume overload. Shunt and respective Sham surgeries were performed in female WT mice. Kidney function was assessed by acute diuretic stress test (A) and measurements of plasma urea (B) in mice six weeks after volume overload and respective controls. $n=6 /$ group; data are presented as mean \pm SEM; n.s.: not significant between Shunt and respective Sham controls using unpaired Student's t-test.

\section{$\underline{\text { 3.2.4 Gene expression }}$}

Using qRT-PCR, cardiac stress markers, such as ANP (Atrial Natriuretic Peptide), BNP (Brain Natriuretic Peptide), $\alpha$-skeletal actin and SERCA-2 $\alpha$ (Sarcoplasmic/Endoplasmic Reticulum Calcium ATPase-2 $\alpha$ ), were assessed at gene expression level in the heart following volume 
overload. Both one and six weeks of volume overload caused significant increases of cardiac ANP (one week: 4.2 fold, six weeks: 2.5 fold, both p<0.05), BNP (one week: 2.6 fold, six weeks: 1.9 fold, both $\mathrm{p}<0.05$ ) and $\alpha$-skeletal actin (one week: 4.0 fold, six weeks: 4.9 fold, both $\mathrm{p}<0.05$ ) compared to respective controls (Fig. 3.6A-C). These changes are commonly referred to as "reactivation of the fetal gene programme", a typical hallmark of heart failure and cardiac stress. In addition, SERCA-2 $\alpha$-mRNA levels were significantly decreased (one week: 0.59 fold, six weeks: 0.64 fold, both $\mathrm{p}<0.01$ ) compared to respective controls (Fig. 3.6D).

A

ANP

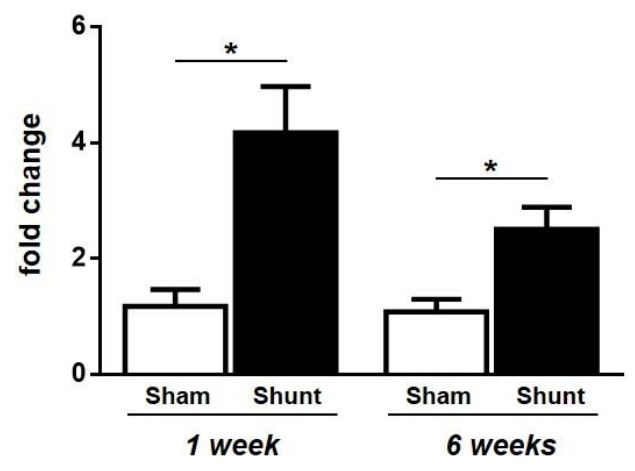

C

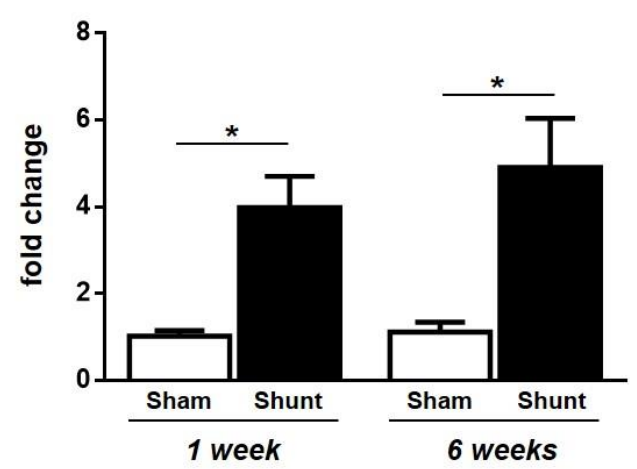

B

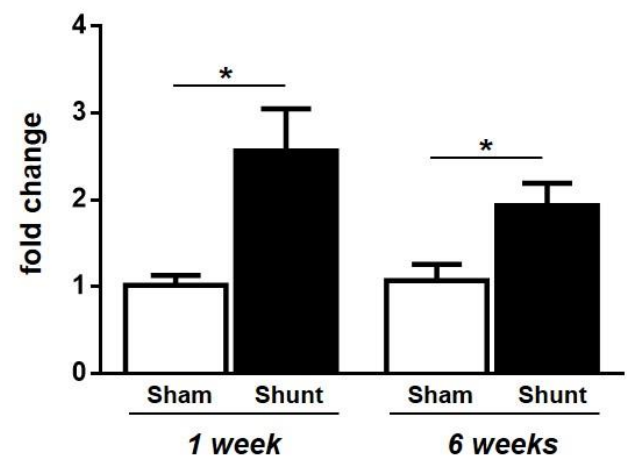

D

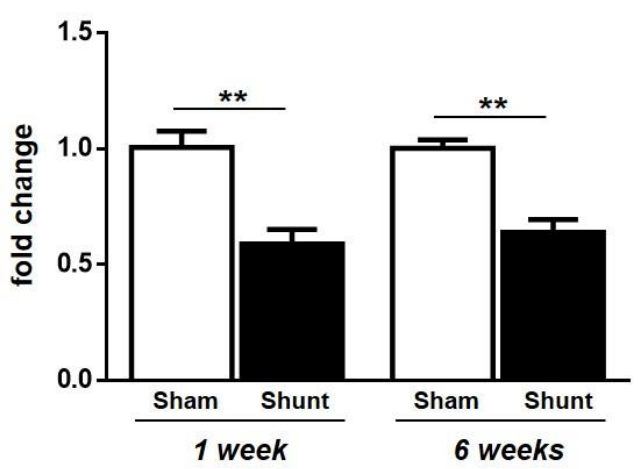

Figure 3.6: Gene expression of cardiac stress markers in mice following volume overload. Shunt and respective Sham surgeries were performed in female WT mice. Cardiac mRNA levels of ANP (Atrial Natriuretic Peptide) (A), BNP (Brain Natriuretic Peptide) (B), $\alpha$-skeletal actin (C) and SERCA-2 $\alpha$ (Sarcoplasmic/Endoplasmic Reticulum Calcium ATPase-2 $\alpha$ ) (D) were measured one and six weeks post surgery. Values are presented as fold change compared to respective Sham controls, GAPDH was used for normalization. $n=4-7 /$ group; data are presented as mean \pm SEM; $* \mathrm{p}<0.05, * * \mathrm{p}<0.01$ between Shunt and respective Sham controls using unpaired Student's t-test. 


\subsection{Non-invasive assessment of cardiac function in mice during pressure and volume overload in mice using state-of-the-art echocardiography}

Using advanced state-of-the-art echocardiographic techniques, systolic and diastolic function in mice were assessed following pressure and volume overload. Pressure overload was induced by six weeks of abdominal aortic banding (AAB) and volume overload through one week of Shunt. These two time points were chosen in order to specifically assess cardiac function under very different haemodynamic conditions, i.e. long-term pressure versus short-term volume overload. This was meant to give new insights into cardiac physiology during haemodynamic load and to evaluate various echocardiographic parameters used in humans with respect to their significance and relevance in mice.

\subsubsection{Systolic function}

Systolic function in mice was assessed using speckle tracking echocardiography after six weeks of chronic pressure overload, induced by abdominal aortic banding (AAB). Heart rates between banded and control animals did not differ (442 vs 450 bpm, p=n.s.) (Fig. 3.7A), the ejection fraction as marker for global systolic function, however, was significantly decreased at this point in banded animals compared to respective Sham controls (34.9 vs 60.6\%, p<0.01) (Fig. 3.7B). Additionally, the longitudinal strain rate was measured, as this parameter has been shown to be more sensitive to detect changes in systolic function ${ }^{97}$. In line with the decrease in ejection fraction, six weeks of $\mathrm{AAB}$ caused a significant reduction in the longitudinal strain rate compared to controls (-4.1 vs $-11.0 \mathrm{~s}^{-1}, \mathrm{p}<0.01$ ) (Fig. 3.7C) indicating systolic dysfunction at this point.

After one week of volume overload, induced by Shunt surgery, mice had similar heart rates compared to Sham controls (435 vs 450 bpm, p=n.s.) (Fig. 3.7D), but were in a hypercontractile stage with increases in both ejection fraction (72.3 vs 56.5\%, p<0.01) (Fig. 3.7E) and longitudinal strain rate $\left(-12.2\right.$ vs $\left.-7.6 \mathrm{~s}^{-1}, \mathrm{p}<0.01\right)$ (Fig. 3.7F). These observations highlight the distinctive differences with respect to systolic function in response to long-term pressure and short-term volume overload. 
A

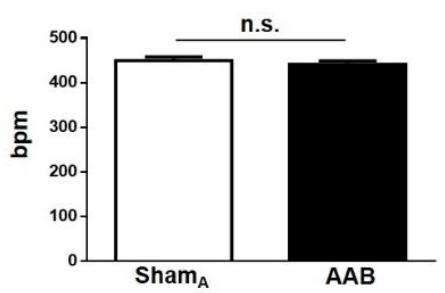

D

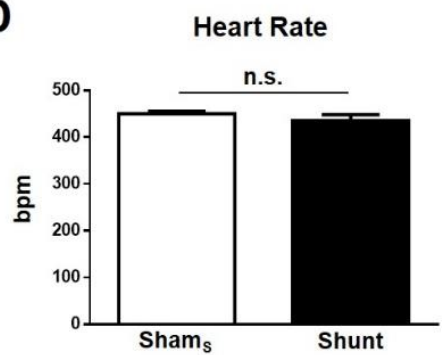

B

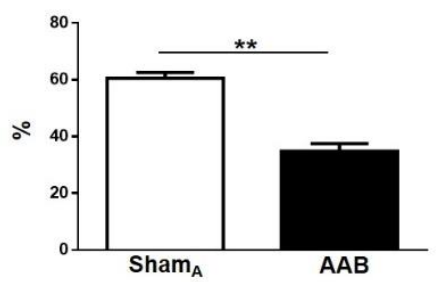

$\mathbf{E}$

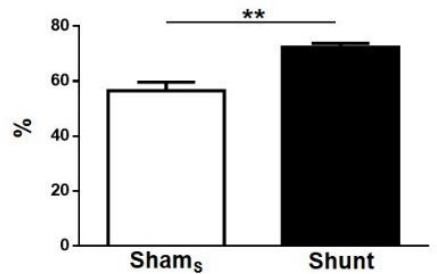

C Longitudinal Strain Rate

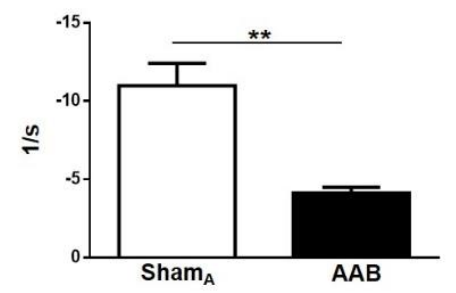

F Longitudinal Strain Rate

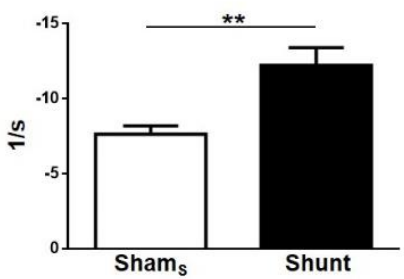

Figure 3.7: Assessment of heart rate and systolic function in murine models of pressure and volume overload using speckle tracking echocardiography. Heart rate (A), ejection fraction (B) and longitudinal strain rate (C) were measured in male WT mice six weeks post AAB (abdominal aortic banding) and in their respective Sham controls $\left(\mathrm{Sham}_{\mathrm{A}}\right)$ using speckle tracking echocardiography. The same analysis was performed for female WT mice one week post Shunt and their Sham controls $\left(\right.$ Sham $\left._{\mathrm{S}}\right)(\mathbf{D}-\mathbf{F}) . \mathrm{n}=6-7 /$ group; data are presented as mean \pm SEM, ** $\mathrm{p}<0.01$, * $\mathrm{p}<0.05$, n.s.: not significant in $\mathrm{AAB}$ or Shunt group compared to their respective Sham controls $\left(\mathrm{Sham}_{\mathrm{A}}\right.$ for $\mathrm{AAB}$, Shams for Shunt) using unpaired Student's t-test.

\subsubsection{Diastolic function}

Diastolic function in mice following long-term pressure and short-term volume overload was analyzed by transmitral flow profiles using pulsed wave Doppler imaging. Fig. 3.8 shows an example of a murine echocardiographic image in the apical 4-chamber view with the sample volume (two yellow horizontal lines) placed in the mitral orifice to acquire the transmitral flow. Transmitral flow profiles can be found in Fig. 3.9 and 3.10. In humans, this technique together with tissue Doppler imaging allows to assess left ventricular relaxation and filling properties by measuring several parameters including isovolumic relaxation time (IVRT), E-wave deceleration time (DT), E/A and E/E'. In the next part of this study, these parameters are evaluated in mice during pressure and volume overload respectively. 


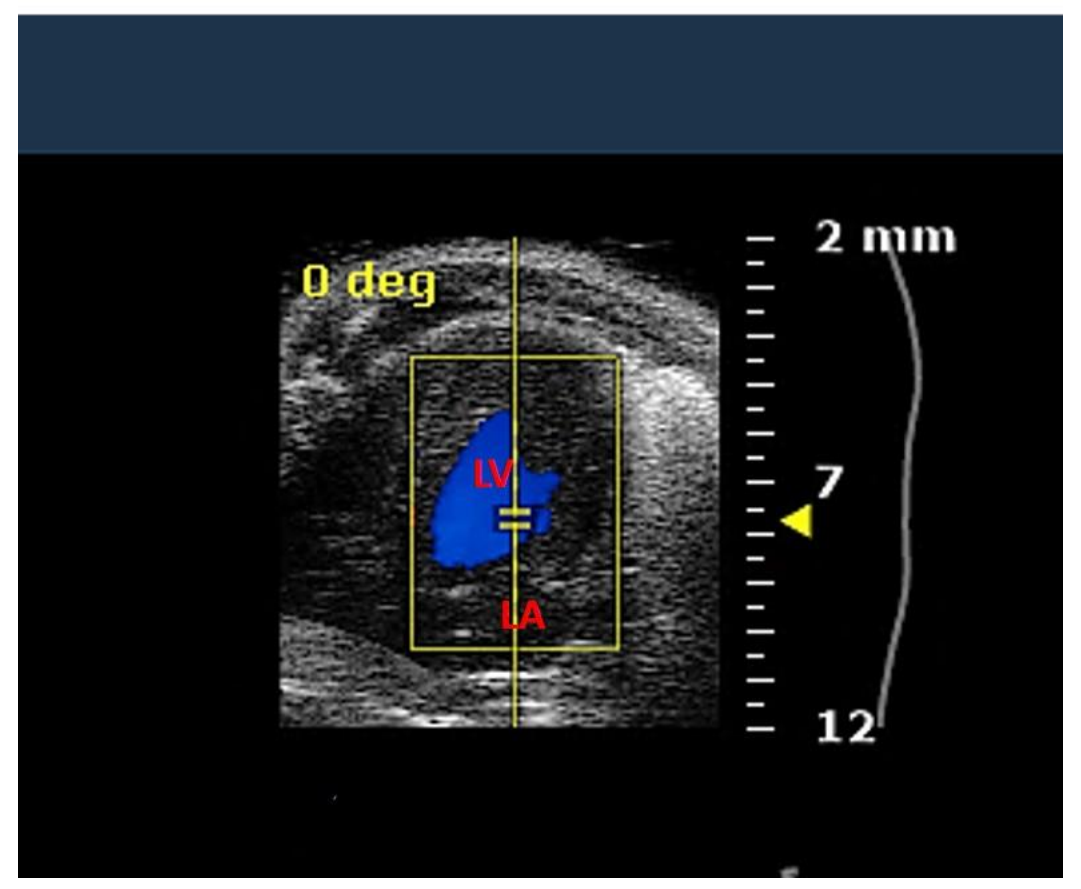

Figure 3.8: Sample placement for transmitral flow analysis. In the apical 4-chamber view, the left atrium (LA in red) and the left ventricle (LV in red) can be visualized. Using pulsed wave Doppler, the sample volume (two horizontal yellow lines) is placed close to the tip of the mitral leaflets in the mitral orifice, the optimal position for acquiring transmitral flow profiles.

\subsubsection{Left ventricular relaxation}

The isovolumic relaxation time (IVRT), measured between aortic valve closure and mitral valve opening, is well-established to be a sensitive parameter for left ventricular relaxation ${ }^{98}$. The progression of diastolic dysfunction typically involves prolongation of the IVRT as sign for an impaired relaxation. At later stages, however, the IVRT becomes shorter due to severe increases in left ventricular filling pressure. Six weeks after AAB, the IVRT was significantly increased compared to Sham controls indicating impaired relaxation (23.3 vs $14.3 \mathrm{msec}$, p<0.01) (Fig. 3.9A,B). However, in contrast to chronic pressure overload, one week of volume overload caused a significant decrease in the IVRT (13.0 vs $17.1 \mathrm{msec}, \mathrm{p}<0.01)$ (Fig. 3.9C,D), presumably due to the Shunt-induced increases in left atrial and left ventricular filling pressure. 
A

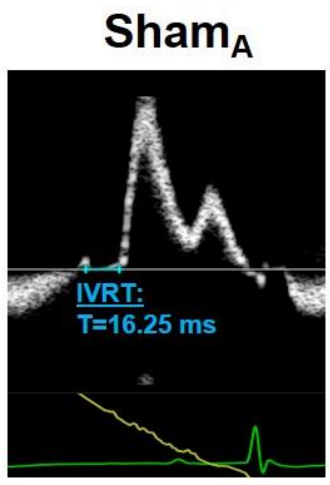

C

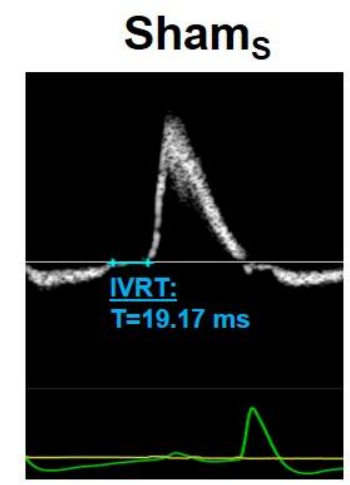

AAB

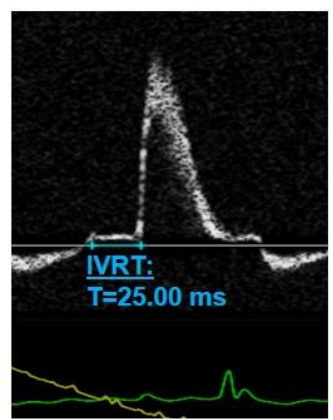

Shunt

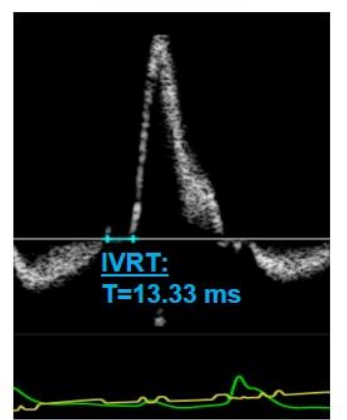

B

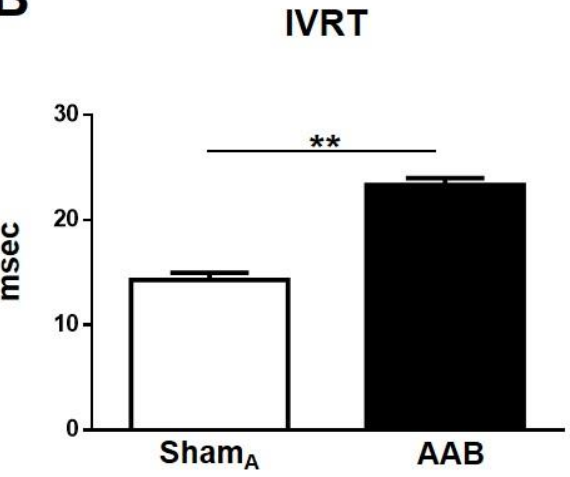

D

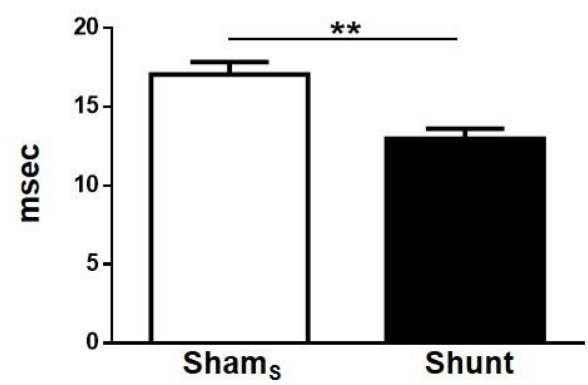

Figure 3.9: Assessment of the isovolumic relaxation time (IVRT) in murine models of pressure and volume overload using echocardiography. $\mathbf{A}$ and $\mathbf{C}$ show representative images for transmitral inflow profiles in mice six weeks post $\mathrm{AAB}(\mathbf{A})$ as well as one week post Shunt $(\mathbf{C})$ and their respective controls (Sham $\mathrm{A}$ and $\mathrm{Sham}_{\mathrm{S}}$ ). Images include exemplary measurements of the IVRT (in blue). Average data for the IVRT are depicted in $\mathbf{B}$ (AAB versus $\mathrm{Sham}_{\mathrm{A}}$ ) and $\mathbf{D}$ (Shunt versus Sham $\mathrm{S}$ ). $\mathrm{n}=7$ /group; data are presented as mean $\pm \mathrm{SEM}$; ** $\mathrm{p}<0.01$ significant in $\mathrm{AAB}$ or Shunt group compared to their respective Sham controls $\left(\operatorname{Sham}_{\mathrm{A}}\right.$ for $\mathrm{AAB}$, Sham $\mathrm{S}$ for Shunt) using unpaired Student's t-test.

\subsubsection{Left ventricular filling}

In humans, the ratio between early and late filling velocities, known as E/A, as well as the Ewave deceleration time (DT) are important parameters to assess diastolic function ${ }^{89}$. In this study, however, these parameters could not be evaluated consistently. Heart rates in mice were between 400 and 500 beats per minute, which in most cases led to fusion of the E- and A-wave, depicted in the representative images of the transmitral inflow profile in Fig. 3.10B,D,E. The clear separation of E- and A-wave, as illustrated in Fig. 3.10A, was exceptional.

The E/E' ratio, a parameter which correlates with left ventricular filling pressure in humans ${ }^{92}$, was determined in mice following volume and pressure overload using transmitral and tissue 
Doppler. After six weeks of abdominal aortic banding, the E/E' ratio increased significantly compared to its respective Sham controls indicating increased filling pressure (48.5 vs 36.5, p <0.01) (Fig. 3.10A-C). In the Shunt model, however, the E/E' ratio did not change (33.7 vs 29.8, p=n.s.) (Fig. 3.10D-F), even though an increase in volume load is known to increase left atrial and therefore left ventricular filling pressure ${ }^{99,100}$. An additional parameter was therefore measured to non-invasively assess the left ventricular filling pressure in mice qualitatively. As the left atrial volume is used in humans to indirectly assess filling pressures ${ }^{89}$, the left atrial area was measured in mice as estimate using the apical 4-chamber view. Indeed, the left atrial area significantly increased in both haemodynamic stress models. Six weeks of AAB caused an increase from 2.3 to $4.3 \mathrm{~mm}^{2}$ (p<0.01) (Fig. 3.11A,B) and one week of Shunt from 3.4 to 6.5 $\mathrm{mm}^{2}(\mathrm{p}<0.01)$ (Fig. 3.11C,D) compared to respective controls.

A

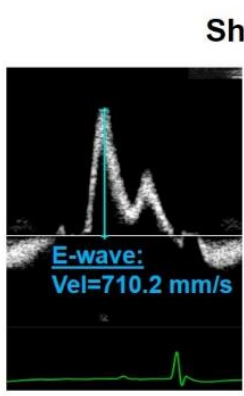

Sham $_{A}$

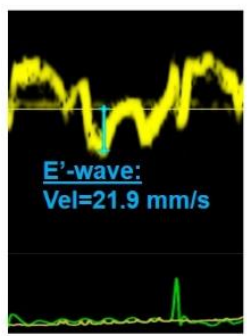

D
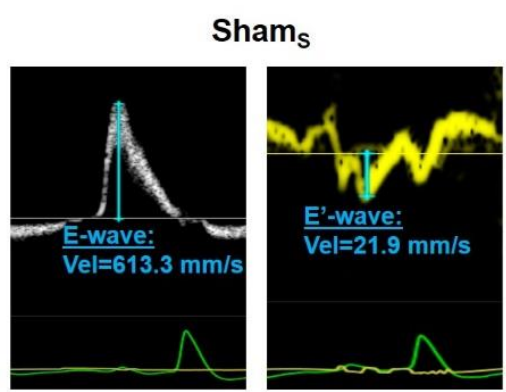

B

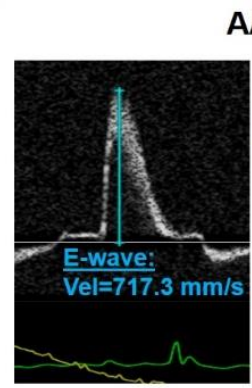

AAB

E

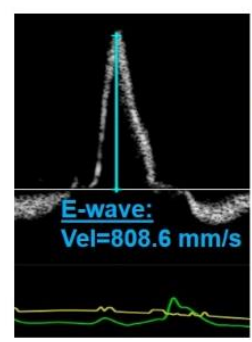

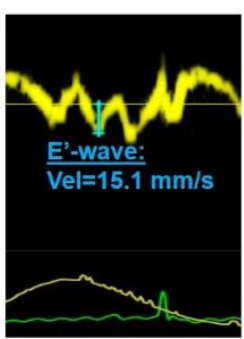

C

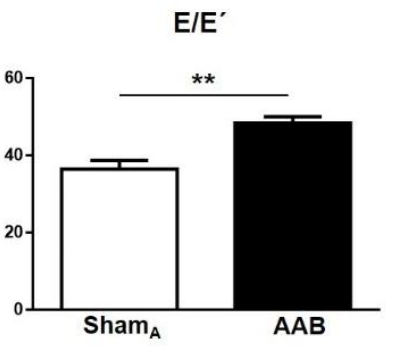

$\mathbf{F}$

$E / E^{\prime}$

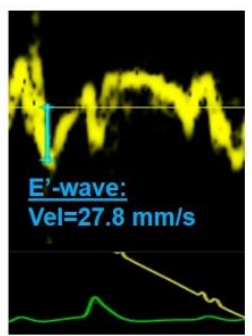

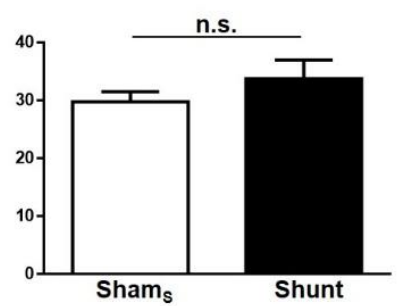

Figure 3.10: Assessment of the $E / E$ ' ratio in murine models of pressure and volume overload using echocardiography Representative echocardiographic images in mice are shown for transmitral (left image in A, B, D, E) and tissue Doppler (right image in A, B, D, E) flow profiles six weeks after AAB as well as one week post Shunt (A, B, D, E). Images include exemplary measurements of the E- and E'-wave velocity after AAB (A, B) and Shunt $(\mathbf{D}, \mathbf{E})$ as well as their respective controls $\left(\mathrm{Sham}_{\mathrm{A}}\right.$ for $\mathrm{AAB}, \mathrm{Sham}_{\mathrm{S}}$ for Shunt). Average data for the E/E' ratios are shown in $\mathbf{C}\left(\mathrm{AAB}\right.$ versus $\mathrm{Sham}_{\mathrm{A}}$ ) and $\mathbf{F}$ (Shunt versus Sham $\mathrm{S}$ ). $\mathrm{n}=7$ /group; data are presented as mean $\pm \mathrm{SEM}$; ** $\mathrm{p}<0.01$, n.s.: not significant in $\mathrm{AAB}$ or Shunt group compared to their respective Sham controls $\left(\mathrm{Sham}_{\mathrm{A}}\right.$ for $\mathrm{AAB}$, Shams for Shunt) using unpaired Student's t-test. 
A

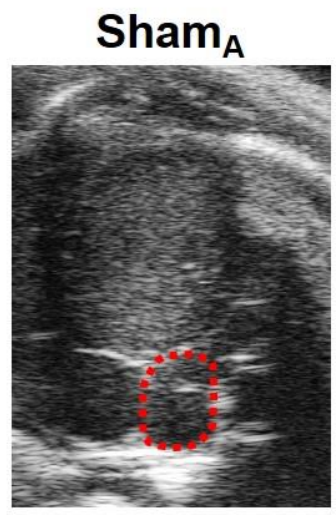

C

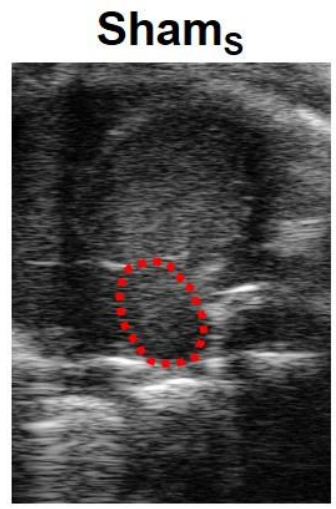

AAB

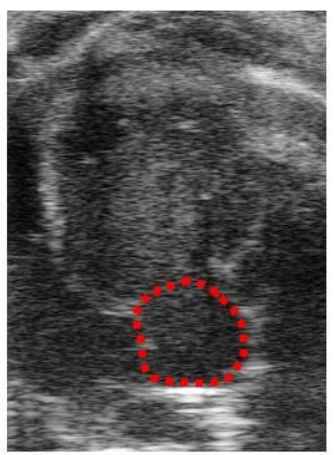

Shunt

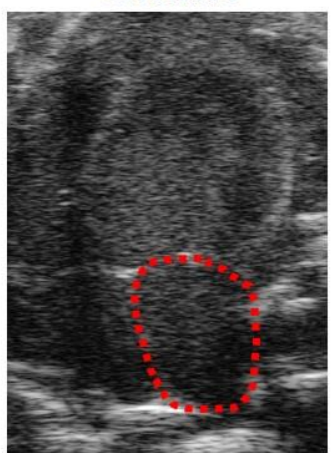

B

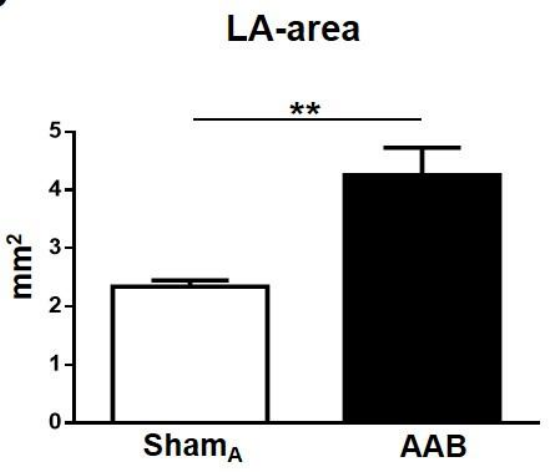

D

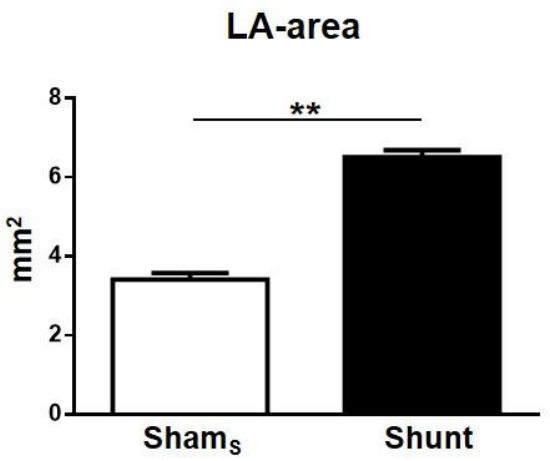

Figure 3.11: Assessment of the left atrial area in murine models of pressure and volume overload using echocardiography. $\mathbf{A}$ and $\mathbf{C}$ show representative images in the apical 4-chamber view to measure the left atrial area (LA-area) in mice six weeks post AAB (A) and one week post Shunt (B), compared to their respective Sham controls (Sham $\mathrm{A}$ for AAB, Shams for Shunt). The left atrium is encircled with red dots. Average data for the left atrial area are depicted in $\mathbf{B}$ (AAB versus Sham ${ }_{A}$ ) and $\mathbf{D}$ (Shunt versus Shams). $n=6-7 /$ group; data are presented as mean \pm SEM; ** $p<0.01$ in AAB or Shunt group compared to their respective Sham controls (Sham $\mathrm{A}$ for AAB, Shams for Shunt) using unpaired Student's t-test.

\subsubsection{Diastolic strain}

Furthermore, diastolic properties were assessed using speckle tracking analysis. Using the InVevo2100 1.5.0 Image Software, the peak longitudinal strain rate during early filling can automatically be determined using the "reverse peak" option, as described previously in Materials and Methods (Fig. 2.3). The longitudinal strain rate during early filling, named reverse longitudinal strain rate, has been shown to be related to left ventricular relaxation and stiffness as well as left atrial pressure and can be altered by changes in preload ${ }^{94,101}$. Thus, six weeks of abdominal aortic banding caused a significant decrease of this parameter compared to respective 
controls (4.6 vs $\left.9.5 \mathrm{~s}^{-1}, \mathrm{p}<0.01\right)$ (Fig. 3.12A,B) presumably due to impaired relaxation. After one week of Shunt, however, this parameter was significantly increased (17.3 vs $\left.9.2 \mathrm{~s}^{-1}, \mathrm{p}<0.01\right)$ (Fig. 3.12C,D), which is in line with an augmented left ventricular filling pressure.

A

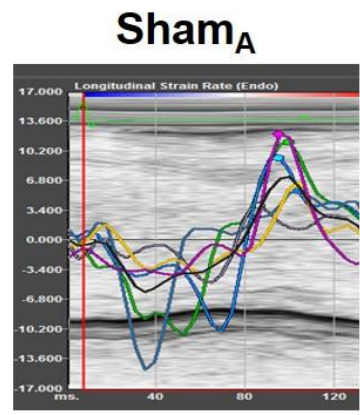

C

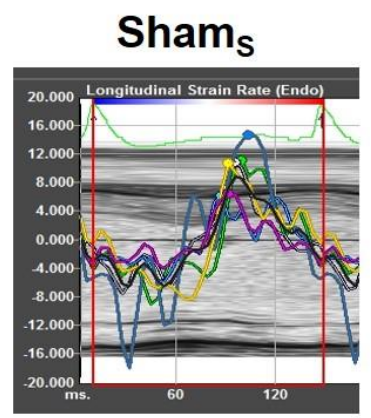

AAB

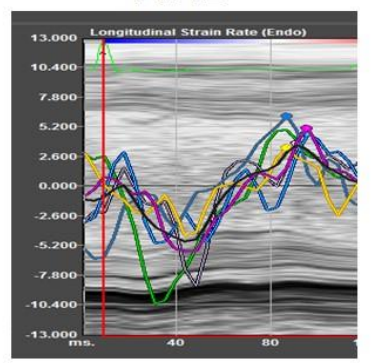

Shunt

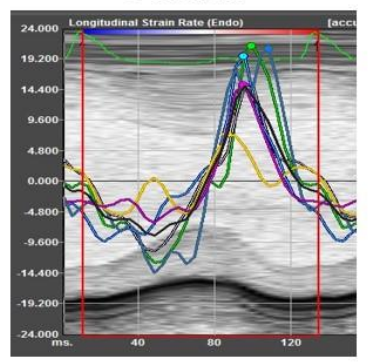

B

Reverse Longitudinal Strain Rate

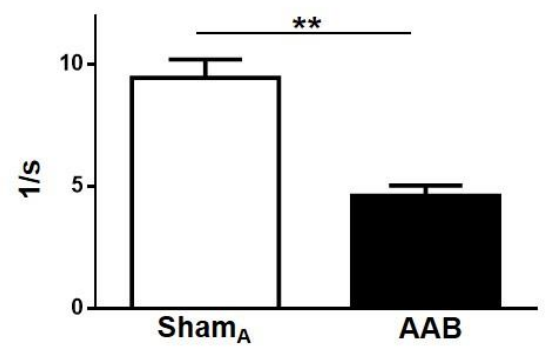

D

Reverse Longitudinal Strain Rate

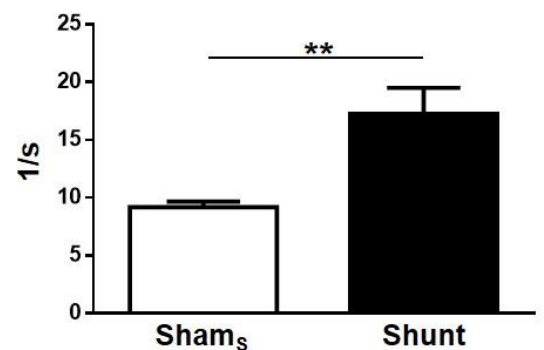

Figure 3.12: Assessment of the reverse longitudinal strain rate in murine models of pressure and volume overload using speckle tracking echocardiography. Using speckle tracking analysis, the reverse longitudinal strain rate was measured six weeks post $\mathrm{AAB}$ and one week post Shunt and their respective Sham controls. Representative images and average data are shown in A-B ( $\mathrm{AAB}$ versus $\mathrm{Sham}_{\mathrm{A}}$ ) and $\mathbf{C}-\mathbf{D}$ (Shunt versus Shams). n=6-7/group; data are presented as mean $\pm \mathrm{SEM} ; * * \mathrm{p}<0.01$ in $\mathrm{AAB}$ or Shunt group compared to their respective Sham controls (Sham ${ }_{\mathrm{A}}$ for AAB, Shams for Shunt) using unpaired Student's t-test.

\subsubsection{Systolic and diastolic function following transverse aortic constriction}

All the previously mentioned systolic and diastolic parameters were also measured in mice after two weeks of transverse aortic constriction (TAC), a severe model to induce pressure overload. Thus, all parameters could be evaluated in terms of their consistency between different models. Indeed, two weeks of transverse aortic constriction induced similar changes to six weeks of abdominal aortic banding. Compared to controls, the ejection fraction decreased from 65.5 to $36.0 \%$ (p<0.01) (Fig.3.13A) with significant increases in the IVRT (14.7 vs $11.3 \mathrm{msec}$; p<0.05) 
(Fig. 3.13B), E/E' (62.6 vs 47.8, p<0.05) (Fig. 3.13C) and left atrial area (6.8 vs $3.3 \mathrm{~mm}^{2}$, p <0.01) (Fig. 3.13D). The left atrial area increase of $106 \%$ matched the $110 \%$ increase in left atrial weight versus tibia length ratio $(0.40$ vs $0.19 \mathrm{mg} / \mathrm{mm}, \mathrm{p}<0.01)$ two weeks after TAC compared to controls (Fig. 3.13E). The reverse longitudinal strain rate in mice was decreased from 11.6 in controls to $7.2 \mathrm{~s}^{-1}$ after two weeks of transverse aortic constriction (Fig. 3.13F). The similar change in parameters after chronic abdominal aortic banding and transverse aortic constriction emphasize consistency of the measured and evaluated parameters between different animal models of a similar haemodynamic stress.

A

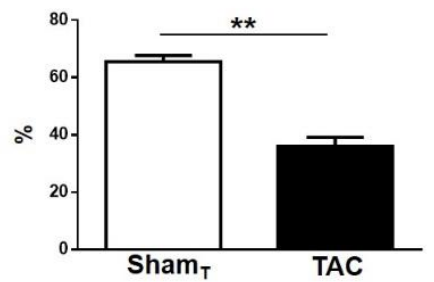

D

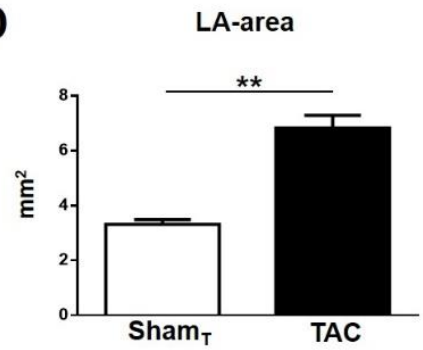

B

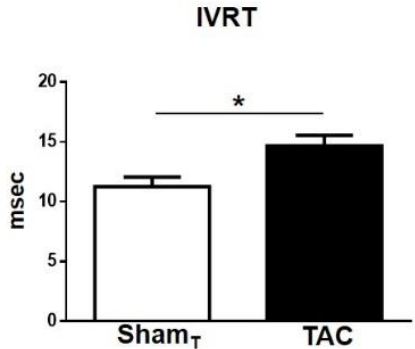

$\mathbf{E}$

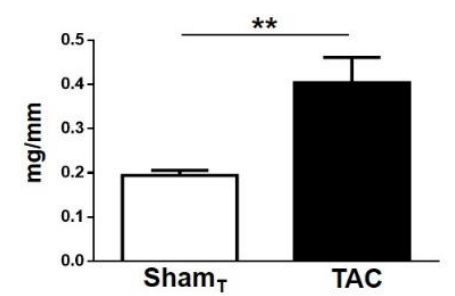

C

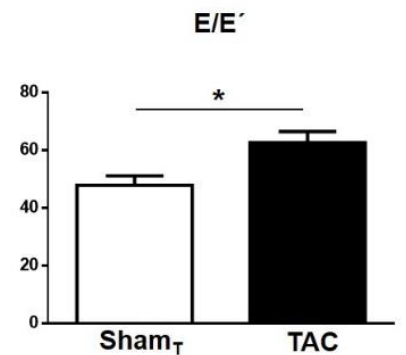

F Reverse Longitudinal Strain Rate

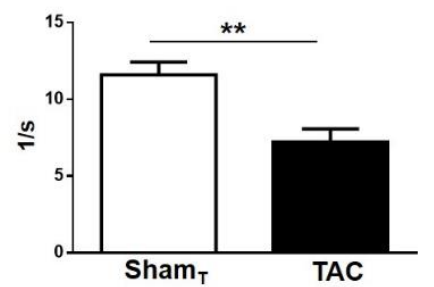

Figure 3.13: Assessment of systolic and diastolic function in mice two weeks after transverse aortic constriction (TAC). Using echocardiography, ejection fraction (A), IVRT (B), E/E' (C), left atrial area (LA-area) (D), left atrial weight (LA Weight) versus tibia length ratio (E) and the reverse longitudinal strain rate (F) were measured in mice two weeks after TAC and in their respective Sham controls $\left(\mathrm{Sham}_{\mathrm{T}}\right)$. n=6-7/group; data are presented as mean $\pm \mathrm{SEM} ; * \mathrm{p}<0.05$, ** $\mathrm{p}<0.01$ between TAC and respective Sham controls $\left(\mathrm{Sham}_{\mathrm{T}}\right)$ using unpaired Student's t-test.

\subsubsection{Correlation of diastolic parameters with cardiac hypertrophy following pressure overload}

It was analyzed, whether the aforementioned diastolic parameters correlate with the extent of cardiac hypertrophy in both pressure overload models $\mathrm{AAB}$ and TAC. Compared to their respective Sham controls $\left(\mathrm{Sham}_{\mathrm{A}}\right.$ for abdominal aortic banding and Sham $\mathrm{T}_{\mathrm{T}}$ for transverse aortic constriction), percentage changes of IVRT, E/E', left atrial area and reverse longitudinal strain 
rate were correlated with the relative increase in heart to body weight (HW/BW) ratio in mice subjected to either six weeks of abdominal aortic banding or two weeks of transverse aortic constriction using linear regression analysis. The relative increases in IVRT and E/E' as well as the relative decrease in the reverse longitudinal strain rate following pressure overload showed significant, positive correlations with the increase in HW/BW ratios. The strongest correlation with cardiac hypertrophy was found in the $E / E^{\prime}$ increase $\left(r^{2}=0.74, p<0.01\right)$ (Fig. 3.14B), followed by the increase in IVRT $\left(\mathrm{r}^{2}=0.65, \mathrm{p}<0.01\right.$ ) (Fig. 3.14A) and the decrease in the reverse longitudinal strain rate $\left(\mathrm{r}^{2}=0.43, \mathrm{p}<0.05\right)$ (Fig. 3.14D). There was no correlation between the increase in HW/BW ratio and the relative left atrial area increase $\left(r^{2}<0.01, p=0.79\right)$ (Fig. 3.14C).

A

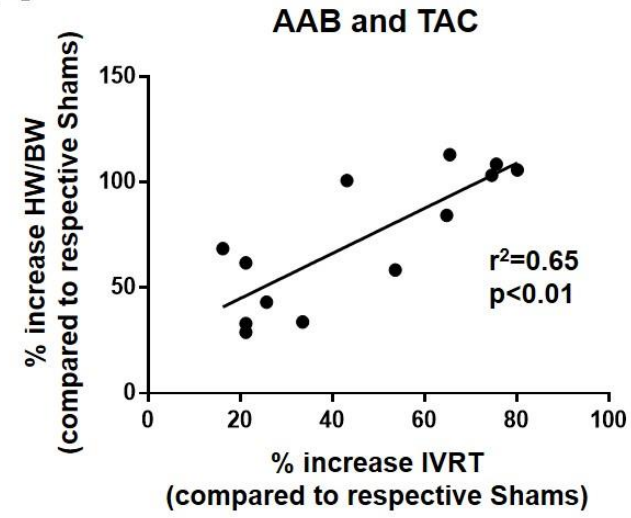

C

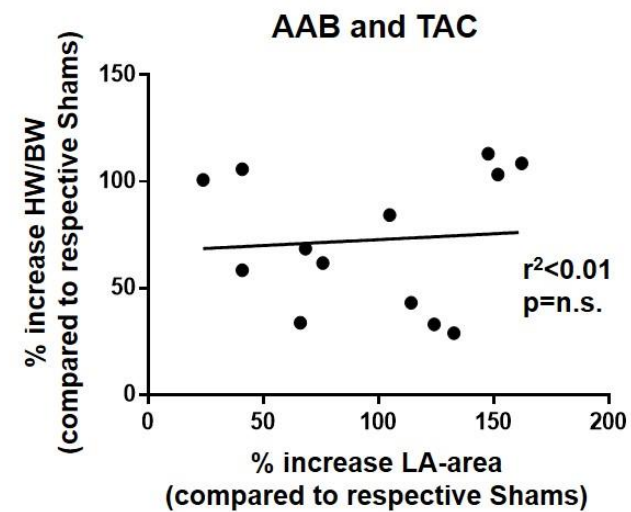

B

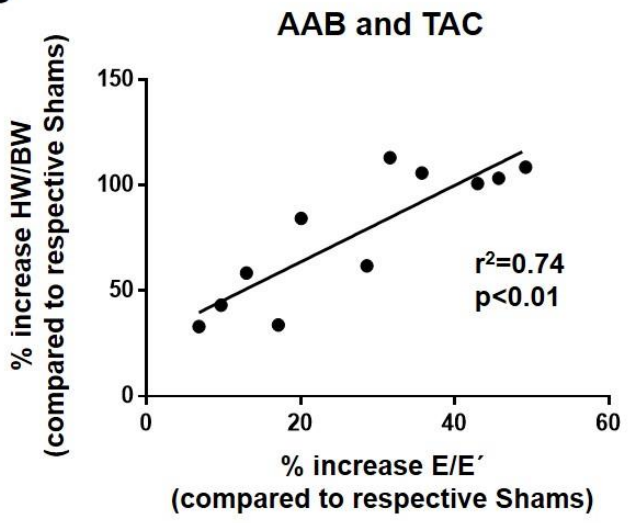

D

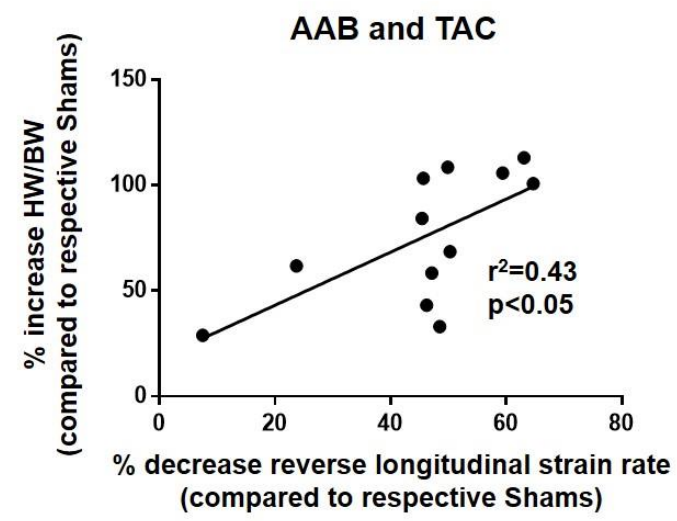

Figure 3.14: Correlation of diastolic parameters and cardiac hypertrophy in murine models of pressure overload. Compared to respective Sham controls, relative changes in IVRT (A), E/E' (B), left atrial area (C) and the reverse longitudinal strain rate (D) were correlated with the relative increase in heart to body weight ratio (HW/BW) for each mouse subjected to either six weeks of AAB or two weeks of TAC. $n=11$-13/group, coefficients of determination $\left(\mathrm{r}^{2}\right)$ were determined and $\mathrm{p}$-values $<0.05$ indicate a significant deviation from zero using linear regression analysis. 


\subsection{Discussion}

The aortocaval fistula model (Shunt) was established and used in this study to induce volume overload in mice. Volume overload is typically associated with eccentric cardiac hypertrophy, which includes increases in ventricular dimensions as well as cardiac weight. Indeed, one week of volume overload caused significant increases in both readouts, which were even more pronounced after six weeks (Fig. 3.1, 3.3; Table 3.1). Systolic function, assessed via ejection fraction measurements using echocardiography, was well-preserved at an early stage after one week, and only slightly worse compared to Sham controls after six weeks of volume overload (Fig. 3.2). Moderate increases of lung weight versus tibia length ratio as sign for pulmonary congestion was detected only after six weeks of volume overload (Fig. 3.4B), again indicating mild cardiac dysfunction. These findings are in line with previous reports in mice showing only slight deterioration of systolic function after long-term volume overload with decreased fractional shortening and increased lung weight ${ }^{27,75}$. Despite early development of cardiac hypertrophy, this model initially seems to reflect a more physiological response with well-preserved systolic function (Fig. 3.2B,C; Table 3.1). This is supported by one study showing no signs of cardiac fibrosis and inflammation in mice one week after Shun ${ }^{27}$. The reactivation of the fetal gene programme, however, does not fit into the scheme of an initial physiological response. The detected increases in ANP, BNP, $\alpha$-skeletal actin and decreased SERCA-2 $\alpha$-mRNA levels (Fig. 3.6) already after one week suggest cardiac stress and are typically associated with transition to heart failure. Thus, the aortocaval fistula induces a pathological stimulus in the heart, which, for unknown reasons, can be compensated very well over a relatively long period of time without development of severe cardiac dysfunction in mice. The reactivation of the fetal gene programme in response to volume overload also seems to be dependent on the mouse strain used. This study uses C57B1/6 mice, whereas the previously mentioned study by Toischer et al. performed their experiments in $\mathrm{FVB} / \mathrm{N}$ mice. Interestingly, they did not find differences in gene expression levels of BNP and SERCA-2 $\alpha$ one week after Shunt suggesting a strain-dependent response to volume overload. The decrease in average kidney weight versus tibia length ratio after both one and six weeks of volume overload in mice (Fig. 3.4D) could not be attributed to impaired renal function, as diuretic stress tests and plasma urea levels gave similar results compared to Sham controls (Fig. 3.5). In rats, the aortocaval fistula was also shown to cause reductions in kidney weight ${ }^{102}$. If the increase in cardiac output cannot compensate for the blood flow through the fistula 
anymore, perfusion of peripheral organs is reduced. Thus, the kidney weight reduction was attributed to a Shunt-mediated decrease in renal blood perfusion.

Taken together, the aortocaval fistula in mice is a unique model as it progressively induces eccentric hypertrophy with early reactivation of the fetal gene programme, but only mild systolic impairment at late time points.

In humans, three essential conditions are necessary to diagnose diastolic heart failure: 1. presence of symptoms of congestive heart failure such as fatigue and dyspnea, 2. presence of normal or mildly abnormal systolic function and 3. evidence of left ventricular diastolic dysfunction. Even though invasively acquired measurements using cardiac catheterization provide the clearest evidence of diastolic dysfunction, echocardiography has become a valuable, inexpensive tool to assess diastolic function in a non-invasive manner. According to the European Society of Cardiology, the central echocardiographic parameter in humans to diagnose diastolic dysfunction is the ratio of early filling velocity and early diastolic annular velocity, commonly known as $\mathrm{E} / \mathrm{E}^{\prime 89}$. This value correlates with the left ventricular filling pressure, which increases as diastolic dysfunction progresses ${ }^{92}$. The ratio of early filling to late filling velocity E/A, the E-wave deceleration time and the left atrial volume index are recommended to use if the $\mathrm{E} / \mathrm{E}$ ' ratio is between 8 and 15 and therefore only suggestive of diastolic dysfunction. The isovolumic relaxation time (IVRT) as well as diastolic strain analysis are not part of the algorithm proposed by the European Society of Cardiology ${ }^{89}$.

In mice, however, an algorithm for the assessment of diastolic properties using echocardiography does not exist, despite many cardiovascular research studies being carried out using mice. Therefore, different diastolic parameters were evaluated in this study with respect to their applicability and relevance in mice using two models of chronic pressure (abdominal aortic banding $\mathrm{AAB}$ and transverse aortic constriction $\mathrm{TAC}$ ) as well as one volume overload (Shunt) model. This is the first study in mice to compare diastolic parameters in such a range of different haemodynamic, cardiac stress models.

Even though the IVRT is not directly part of the algorithm to assess diastolic dysfunction in humans anymore, in mouse models of restrictive cardiomyopathy the increase in IVRT was a very sensitive echocardiographic sign of diastolic dysfunction ${ }^{103,104}$. This is in line with the increase in IVRT after six weeks of AAB and two weeks of TAC (Fig. 3.9B, 3.13B). This might be at least partially due to the chronic pressure overload-induced cardiac fibrosis, a main contributor to impaired relaxation ${ }^{27,73,105}$. Volume overload (Shunt), however, increases the 
ventricular filling and the preload ${ }^{27}$, which leads to the observed decrease in IVRT (Fig. 3.9D) after one week. Broadly speaking, in a volume overload setting, the left ventricle just has less time to relax. Under these circumstances it is difficult to draw a conclusion with respect to the functional relevance of the IVRT decrease. This is due to volume overload-induced preload being a confounding influence on the IVRT as it opposes a potential left ventricular relaxation impairment. This is one of its limitations to carefully assess relaxation ${ }^{106}$. However, it is a useful, consistent parameter which is easy to obtain and can add valuable information, providing the physiological background is fully understood.

The ratio of early and late filling velocities (E/A) as well as the deceleration time of the E-wave (DT) are part of the recommended parameters to assess diastolic function in humans ${ }^{89,} 106$. Additionally, these two parameters are also commonly used in murine studies ${ }^{107-109}$. Here, however, it is reported to be very challenging to measure these values as high heart rates in mice led to fusion of E- and A-wave (Chapter 3.3.2.2). This subsequently did not allow to separately measure them. This has also been reported by others before ${ }^{110}$. A clear separation, as shown in the representative transmitral flow profile in Fig. 3.10A (left image), was exceptional; thus, it is not recommended to use this parameter routinely in mice as it cannot be measured consistently.

The ratio of peak early filling to annular tissue velocity (E/E') is the most important parameter to assess diastolic dysfunction in humans using echocardiography ${ }^{89}$ and is also most commonly used in murine studies, which focus on diastolic function ${ }^{108,111,112}$. In both chronic pressure overload models $\mathrm{AAB}$ and $\mathrm{TAC}$, the $\mathrm{E} / \mathrm{E}$ ' was increased (Fig. 3.10C, 3.13C). This is in accordance with an increased left ventricular filling pressure. Volume overload, on the other hand, did not change E/E' (Fig. 3.10F), although it is known to increase the left ventricular filling pressure $^{99,100}$. One explanation for these different findings is the physiology behind the E'. In a chronic pressure overload setting, E' mainly reflects the delayed left ventricular relaxation as it occurs after equilibration of left atrial and left ventricular pressures. Thus, E' decreases after chronic pressure overload (see representative image in Fig. 3.10A,B: E' decreases from 21.9 in controls to $15.1 \mathrm{~mm} / \mathrm{s}$ after six weeks of $\mathrm{AAB}$ ) and as a consequence, the $\mathrm{E} / \mathrm{E}$ ' ratio increases (Fig. 3.10C). In volume overload, however, the E' peak is reached quicker due to a faster relaxation. In this situation, E' mainly reflects the left atrial to left ventricular pressure gradient $^{113}$, which is increased in volume overload. This leads to an increased E' peak velocity (see representative image in Fig. 3.10D,E: $E^{\prime}$ increases from 21.9 in controls to $27.8 \mathrm{~mm} / \mathrm{s}$ after one week of Shunt). Therefore, even though the early filling peak velocity (E) increases (see 
representative image in Fig. 3.10D,E: E increases from 613.3 in controls to $808.6 \mathrm{~mm} / \mathrm{s}$ after one week of Shunt), the E/E' ratio remains unchanged (Fig. 3.10F).

The longitudinal strain rate during early filling (reverse longitudinal strain rate), a parameter that has rarely been described in human or mouse studies, has been shown to share similar properties of $\mathrm{E}^{, 94}$. Indeed, both chronic pressure overload models AAB and TAC showed a decrease (Fig. $3.12 \mathrm{~B}, 3.13 \mathrm{~F}$ ), whereas volume overload caused an increase in the reverse longitudinal strain rate (Fig. 3.12D), presumably for similar reasons as previously mentioned for E'.

In humans, the left atrial volume is an important, valuable parameter to assess diastolic function $^{89,114,115}$. Similarly, in mice the left atrial area is a technically simple measurement to indirectly assess the left ventricular filling pressure. However, it is not very frequently measured in murine studies ${ }^{116}$. Using the apical 4-chamber view, the left atrial area was increased in both chronic pressure overload (Fig. 3.11B, 3.13D) and volume overload (Fig. 3.11D) models, which is in line with increased filling pressures in all models. Thus, left atrial area seems to be a consistent, valid measurement throughout different cardiac stress models to assess diastolic function in mice.

It has been known for more than two decades that pressure overload-induced hypertrophy leads to diastolic impairment in humans ${ }^{117}$. This is the first study to correlate a variety of diastolic parameters with the level of cardiac hypertrophy in mice following pressure overload (AAB and TAC). Significant, positive correlations were found between the relative E/E', IVRT, reverse longitudinal strain rate changes and the relative increase in heart to body weight ratios (HW/BW), each compared to respective Sham controls $\left(\operatorname{Sham}_{\mathrm{A}}\right.$ for $\mathrm{AAB}$ and $\mathrm{Sham}_{\mathrm{T}}$ for TAC) (Fig. 3.14A,B,D). This in particular emphasizes the relevance and significance of these three parameters in order to assess diastolic function in mice. A human study by Müller-Brunotte et al. confirmed these findings using echocardiography in hypertensive patients ${ }^{118}$. They report significant, positive correlations of diastolic parameters, such as IVRT, with left ventricular hypertrophy. Between the left atrial area and the HW/BW ratio increase, however, no correlation was observed (Fig. 3.14C) suggesting a more qualitative rather than quantitative role for the left atrial area in the diastolic assessment in mice.

Taken together, this study reveals in a variety of different pathophysiological models that most of the commonly used human, diastolic parameters can be sufficiently assessed in mice using stateof-the-art echocardiography. However, some measurements are more useful than others due to technically challenging conditions in mice (e.g. small organ size, high heart rates). For instance, 
E/A and DT were inconsistent in terms of their measurability and would therefore not be recommended. This study also emphasizes the importance of the physiology behind these distinctive diastolic parameters in order to interpret them correctly. Furthermore, a combination of several parameters is needed in order to obtain a detailed understanding of the diastolic properties in mice. In particular, E/E', IVRT and the reverse longitudinal strain rate seem to be sensitive markers to detect diastolic dysfunction in mice, as they also correlate with the amount of pressure overload-induced cardiac hypertrophy. Taking all of this into consideration, diastolic assessment in mice should become part of the routinely performed readouts to analyze cardiac function. 


\section{Results II: Cardiac metabolism in murine models of volume and pressure overload}

\subsection{Introduction}

Pressure and volume overload induce different patterns of cardiac remodelling, i.e. concentric versus eccentric remodelling. In concentric remodelling, cardiomyocytes grow in width due to sarcomeres being added in parallel. Eccentric remodelling is characterized by sarcomere addition in series, which leads to cardiomyocyte lengthening. Apart from the different remodelling patterns, differences in intracellular signalling including metabolic pathways are likely to be involved in the cardiac response to both haemodynamic stresses. In order to study these, murine models of pressure and volume overload have been established: the transverse aortic constriction (TAC) model to induce pressure overload and the aortocaval fistula (Shunt) model to mimic volume overload. Indeed, both models have been shown to induce very different cardiac responses in terms of remodelling, function and intracellular signalling ${ }^{27}$. However, they have not yet been carefully characterized and compared with respect to cardiac metabolism. Only a few animal studies focus on cardiac metabolism during volume overload ${ }^{119,120}$, whereas pressure overload-induced changes in cardiac metabolism are more commonly studied. Especially with respect to glucose metabolism, the reported data are often not consistent and even contradictory. One study found decreased glucose uptake and oxidation with unchanged levels of glycolysis in murine hearts following TAC using an isolated working heart perfusion setup ${ }^{121}$. Other studies reported increased glycolysis and glucose uptake in hearts following pressure overload in both rats and mice ${ }^{122,123}$. Additionally, an initial increase in cardiac glucose oxidation in response to TAC was shown in rats, which was normalized in a compensated stage, but progressively decreased as systolic dysfunction occurred ${ }^{124}$. This study by Doenst et al., like many others, base their conclusions on expression profiles of metabolic enzymes and ex vivo experiments in isolated working heart perfusion setups. The ex vivo perfusion method provides the flexibility to trace passages of the substrate through various metabolic pathways under conditions where the perfusate composition and the heart's loading conditions are tightly controlled. However, it clearly does not reflect in vivo conditions, where loading is different, there are basal neurohumoral influences and multiple substrates are usually present in the circulating blood. Thus, infusion of labelled precursors into living organisms is gaining popularity to analyze 
metabolism in vivo. In this context, radiolabelling and heavy atom-labelling need to be distinguished. Both labelling strategies use molecules, in which at least one atom is present as an isotope other than its naturally most abundant one. In the case of radiolabelling, this isotope is radioactive (e.g. ${ }^{18} \mathrm{~F},{ }^{14} \mathrm{C}$ ) and emits radiation during its decay, heavy atom-labelling, however, involves stable isotopes such as ${ }^{13} \mathrm{C}$. Both are common labelling techniques to track a specific isotope through chemical reactions or metabolic pathways respectively. In this study, we focused on isotopic labelling using ${ }^{13} \mathrm{C}$ as it is stable, uncommon in the environment $(1.1 \%$ of the total carbon pool compared to ${ }^{12} \mathrm{C}$ making up $98.9 \%$ ) and non-radioactive. Within a compound, such as glucose or pyruvate, any number of carbons can be substituted at any position to give a range of specifically labelled glucose or pyruvate isotopomers (same number of carbon isotopes but at different positions within the compound). In the course of this study, however, we have used [U$\left.{ }^{13} \mathrm{C}\right]$ glucose, in which all six carbons are uniformly ${ }^{13} \mathrm{C}$-labelled. The majority of studies using an in vivo labelling approach have been carried out to study cancer metabolism in both mice and humans ${ }^{125-127}$. With respect to cardiac metabolism, infusion of labelled tracers into larger animals has been reported. Intracoronary infusion of labelled pyruvate has been conducted in swine models to study cardiac TCA (tricarboxylic acid) cycle metabolism ${ }^{128,}{ }^{129}$, cardiac glucose and fatty acid oxidation were studied in a dog heart failure model via intravenous administration of $\left[\mathrm{U}-{ }^{14} \mathrm{C}\right]$ glucose and $\left[9,10-{ }^{3} \mathrm{H}\right]$ oleate ${ }^{130}$.

Clearly, more work is needed to study cardiac metabolism in vivo under different stress conditions as altering metabolism remains an interesting approach for heart failure therapy development. However, as outlined before, the current debate on metabolic activity including substrate utilization in the heart, especially under stress, is controversial and unclear. This is at least partially due to the usage of various animal models and different experimental setups. Thus, comparing murine models of pressure and volume overload under the same conditions (i.e. animal husbandry, duration of haemodynamic stress, experimental setup) with respect to glucose utilization would give reliable information about haemodynamic load-induced metabolic alterations in the heart. In vivo assessment using a ${ }^{13} \mathrm{C}$-labelling methodology via intravenous infusion of $\left[\mathrm{U}^{13} \mathrm{C}\right]$ glucose in mice followed by NMR-spectroscopy and metabolic pathway analysis is a novel approach in this context. Additionally, expression profiles of metabolic enzymes on mRNA and protein level were analyzed and linked to the in vivo data to get a more comprehensive understanding of differences in cardiac metabolism during pressure versus volume overload. 


\subsection{Basic characterization of cardiac responses to two weeks of pressure and volume overload in mice}

Pressure overload in male WT mice was induced through transverse aortic constriction (TAC), volume overload through aortocaval fistula (Shunt) surgery including respective Sham controls (Sham $_{\mathrm{T}}$ for TAC, Sham $\mathrm{S}$ for Shunt). Before focusing in detail on differences in cardiac metabolism including a novel ${ }^{13} \mathrm{C}$-labelling methodology, the two models had to be generally characterized and compared with respect to changes in left ventricular remodelling and function. The equal duration of two weeks was chosen for both models before transthoracic echocardiography and morphometric analysis were performed.

\subsubsection{Cardiac remodelling, function and hypertrophy}

Transthoracic echocardiography revealed significant differences in cardiac remodelling following two weeks of pressure and volume overload. Volume overload through Shunt surgery caused eccentric remodelling with an increased left ventricular end-diastolic dimension (4.7 vs $4.1 \mathrm{~mm}$, $\mathrm{p}<0.01)$, unchanged septum thickness, slightly increased posterior wall thickness (0.69 vs 0.61 $\mathrm{mm}, \mathrm{p}<0.01)$ and a significant decrease in the relative wall thickness $(0.30$ vs $0.32, \mathrm{p}<0.01)$ compared to respective Sham controls. As expected, two weeks of pressure overload through TAC surgery induced concentric remodelling with an unchanged left ventricular end-diastolic diameter, but an increased septum $(0.87$ vs $0.68 \mathrm{~mm}, \mathrm{p}<0.01)$, posterior wall (0.81 vs $0.62 \mathrm{~mm}$, $\mathrm{p}<0.01)$ and relative wall thickness $(0.40$ vs $0.32, \mathrm{p}<0.01)$ compared to respective controls (Fig. 4.1A-C, Table 4.1). The left ventricular end-systolic dimension, a parameter depending on both contractile function and size of the left ventricle, was increased to a similar extent under both haemodynamic stress conditions compared to respective Sham controls (Table 4.1). 
A

LVEDD

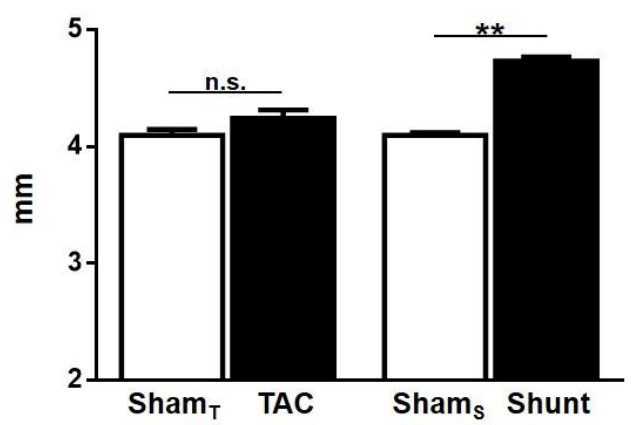

B Septum Thickness

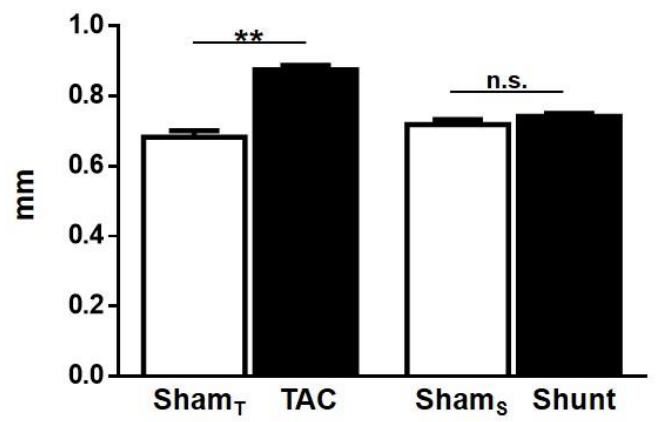

C Relative Wall Thickness

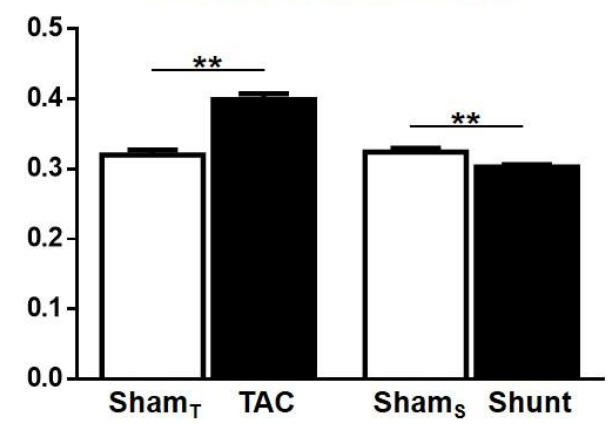

Figure 4.1: Echocardiographic assessment of cardiac remodelling in mice following two weeks of pressure and volume overload. Transverse aortic constriction (TAC) and Shunt surgery were performed in age-matched, male WT mice and compared to respective Sham controls $\left(\mathrm{Sham}_{\mathrm{T}}\right.$ for TAC, Sham $\mathrm{S}$ for Shunt). Transthoracic echocardiography was conducted two weeks after surgery to assess the left ventricular end-diastolic diameter (LVEDD) (A), septum thickness (B), followed by calculation of the relative wall thickness $(\mathbf{C}) . \mathrm{n}=8$-14/group; data are presented as mean $\pm \mathrm{SEM} ; * * \mathrm{p}<0.01$, n.s.: not significant between $\mathrm{TAC} / \mathrm{Sh}$ unt and respective Sham controls using unpaired Student's t-test.

Heart rates were similar in all four groups with only slight increases after TAC and Shunt compared to respective controls (Fig. 4.2A, Table 4.1). However, the ejection fraction as marker for global systolic function was significantly decreased after two weeks of TAC (37.3 vs 50.9\%, $\mathrm{p}<0.01$ ), but remained well-preserved in the volume overload model (53.6 vs 55.1\%, p=n.s) (Fig. 4.2B, Table 4.1). Despite these differences in systolic function and remodelling patterns, the amount of left ventricular hypertrophy, measured by left ventricular weight versus tibia length ratio, was almost identical after two weeks of TAC and Shunt $(+42.3 \%$ after TAC vs $+42.5 \%$ after Shunt, both $\mathrm{p}<0.01$ ) compared to respective Sham controls (Fig. 4.2C, Table 4.1). Thus, volume overload-induced eccentric hypertrophy appears to be better adapted than a comparable 
level of concentric hypertrophy through pressure overload with respect to systolic function. Whether alterations in cardiac metabolism might play a role in these different responses under both haemodynamic stress conditions is investigated in the following part of this Chapter.

A

Heart Rate

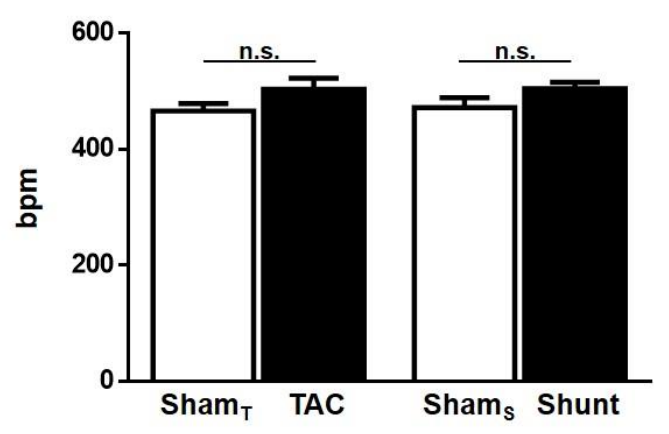

B

Ejection Fraction

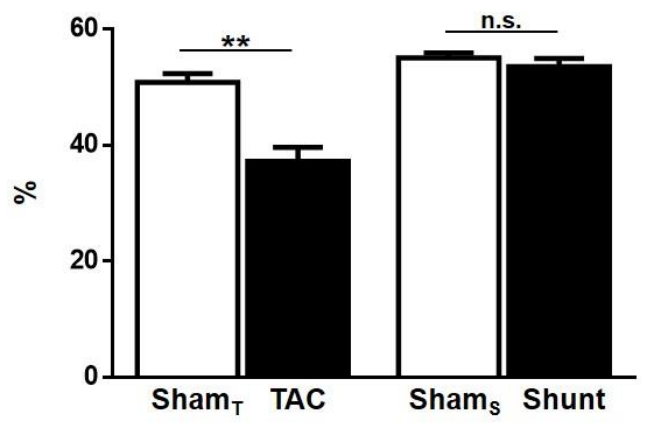

C

LV Weight/Tibia Length

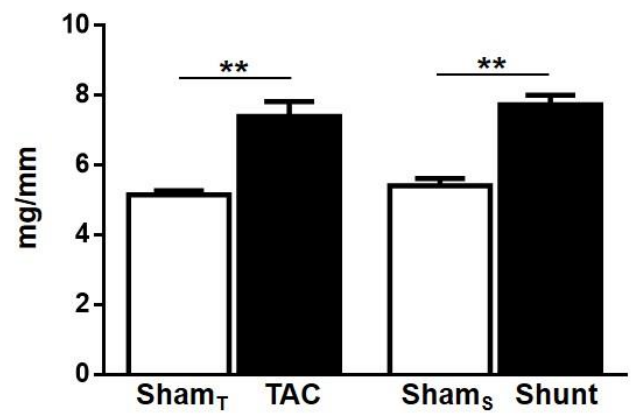

Figure 4.2: Assessment of left ventricular function and hypertrophy in mice following two weeks of pressure and volume overload. Transverse aortic constriction (TAC) and Shunt surgery were performed in age-matched, male WT mice and compared to respective Sham controls $\left(\mathrm{Sham}_{\mathrm{T}}\right.$ for TAC, Sham $\mathrm{S}$ for Shunt). Transthoracic echocardiography was conducted two weeks after surgery to measure the heart rate (A) and ejection fraction (B). The left ventricular (LV) weight for each mouse was assessed post mortem and normalized to the respective tibia length (C). $n=5-14$ /group; data are presented as mean \pm SEM; ** $p<0.01$, n.s.: not significant between TAC/Shunt and respective Sham controls using unpaired Student's t-test. 


\begin{tabular}{|c|c|c|c|c|}
\hline & \multicolumn{2}{|c|}{2 weeks } & \multicolumn{2}{|c|}{2 weeks } \\
\hline & Sham $_{\mathrm{T}}$ & TAC & Sham $_{S}$ & Shunt \\
\hline$n=$ & 8 & 14 & 8 & 11 \\
\hline HR [bpm] & $466 \pm 13.1$ & $504 \pm 19.0$ & $472 \pm 16.9$ & $505 \pm 10.7$ \\
\hline LVID;d [mm] & $4.1 \pm 0.04$ & $4.2 \pm 0.07$ & $4.1 \pm 0.02$ & $4.7 \pm 0.03^{* *}$ \\
\hline LVID;s [mm] & $3.1 \pm 0.06$ & $3.5 \pm 0.10^{* *}$ & $2.9 \pm 0.02$ & $3.4 \pm 0.05^{* *}$ \\
\hline LVV;d $[\mu l]$ & $74.4 \pm 2.1$ & $81.1 \pm 3.3$ & $74.2 \pm 1.0$ & $104.3 \pm 1.9^{* *}$ \\
\hline LVV;s $[\mu \mathrm{l}]$ & $36.8 \pm 2.1$ & $51.8 \pm 3.7^{* *}$ & $33.4 \pm 0.70$ & $48.5 \pm 1.7^{* *}$ \\
\hline Septum [mm] & $0.68 \pm 0.01$ & $0.87 \pm 0.01^{* *}$ & $0.71 \pm 0.01$ & $0.74 \pm 0.01$ \\
\hline Post. Wall [mm] & $0.62 \pm 0.01$ & $0.81 \pm 0.02^{* *}$ & $0.61 \pm 0.01$ & $0.69 \pm 0.01^{* *}$ \\
\hline LV mass [mg] & $75.0 \pm 3.5$ & $108.8 \pm 4.5^{* *}$ & $73.3 \pm 0.82$ & $107.6 \pm 2.1^{* *}$ \\
\hline RWT & $0.32 \pm 0.01$ & $0.40 \pm 0.01^{* *}$ & $0.32 \pm 0.01$ & $0.30 \pm 0.01^{* *}$ \\
\hline$S V[\mu l]$ & $37.6 \pm 0.75$ & $29.6 \pm 1.5^{* *}$ & $40.9 \pm 0.88$ & $55.8 \pm 1.7^{* *}$ \\
\hline EF [\%] & $50.9 \pm 1.5$ & $37.3 \pm 2.4^{* *}$ & $55.1 \pm 0.86$ & $53.6 \pm 1.4$ \\
\hline FS [\%] & $25.7 \pm 0.92$ & $18.0 \pm 1.3^{* *}$ & $28.3 \pm 0.55$ & $27.7 \pm 0.90$ \\
\hline
\end{tabular}

\begin{tabular}{|c|c|c|c|c|}
\hline $\mathrm{n}=$ & 5 & 5 & 5 & 5 \\
\hline $\mathrm{HW} / \mathrm{TL}[\mathrm{mg} / \mathrm{mm}]$ & $6.7 \pm 0.14$ & $9.3 \pm 0.55^{* *}$ & $7.0 \pm 0.23$ & $10.4 \pm 0.41^{* *}$ \\
\hline $\mathrm{LV} / \mathrm{TL}[\mathrm{mg} / \mathrm{mm}]$ & $5.2 \pm 0.12$ & $7.4 \pm 0.42^{* *}$ & $5.4 \pm 0.21$ & $7.7 \pm 0.27^{* *}$ \\
\hline $\mathrm{RV} / \mathrm{TL}[\mathrm{mg} / \mathrm{mm}]$ & $1.2 \pm 0.11$ & $1.3 \pm 0.07$ & $1.2 \pm 0.03$ & $2.0 \pm 0.09^{* *}$ \\
\hline LA/TL $[\mathrm{mg} / \mathrm{mm}]$ & $0.15 \pm 0.02$ & $0.41 \pm 0.06^{* *}$ & $0.15 \pm 0.02$ & $0.32 \pm 0.05^{* *}$ \\
\hline $\mathrm{RA} / \mathrm{TL}[\mathrm{mg} / \mathrm{mm}]$ & $0.17 \pm 0.02$ & $0.18 \pm 0.02$ & $0.23 \pm 0.01$ & $0.33 \pm 0.04^{*}$ \\
\hline
\end{tabular}

Table 4.1: Echocardiographic and morphometric data in mice following two weeks of pressure and volume overload. Transverse aortic constriction (TAC) and Shunt surgery were performed in age-matched, male WT mice and compared to respective Sham controls (Sham $\mathrm{T}$ for TAC, Sham $\mathrm{S}$ for Shunt). Two weeks after surgery, transthoracic echocardiography was performed, followed by removal of hearts and weighing of individual cardiac chambers, each normalized to the respective tibia length (TL). HR: heart rate, bpm: beats per minute, LVID;d: left ventricular diameter in diastole, LVID;s: left ventricular diameter in systole, LVV;d: left ventricular volume in diastole, LVV;s: left ventricular volume in systole, septum: septal wall thickness, post. Wall: posterior wall thickness, RWT: relative wall thickness, SV: stroke volume, EF: ejection fraction, FS: fractional shortening, HW: total heart weight, LV: left ventricular weight, RV: right ventricular weight, LA: left atrial weight, RA: right atrial weight. Data are presented as mean $\pm \mathrm{SEM} ; * \mathrm{p}<0.05, * * \mathrm{p}<0.01$ between TAC/Shunt and respective Sham controls using unpaired Student's t-test. 
4. Results II 


\subsection{Changes in cardiac glucose metabolism in mice two weeks after pressure and volume overload}

Cardiac glucose metabolism in mice following pressure and volume overload was assessed via three different approaches:

1. A novel in vivo ${ }^{13} \mathrm{C}$ glucose-labelling strategy with subsequent analysis of flux into different metabolic pathways using NMR-spectroscopy.

2. Gene expression analysis of enzymes involved in different metabolic pathways.

3. Protein expression analysis of enzymes involved in different metabolic pathways.

A particular focus of this study is on cardiac glycolytic activity, TCA cycle and glutamine metabolism under both haemodynamic stress conditions.

\subsubsection{Glycolysis}

After two weeks of tranverse aortic constriction (TAC), Shunt and respective Sham control surgeries $\left(\mathrm{Sham}_{\mathrm{T}}\right.$ for TAC, Sham $\mathrm{S}$ for Shunt) in male WT mice, $\left[\mathrm{U}-{ }^{13} \mathrm{C}\right]$ glucose was continuously administed intravenously for 30 minutes, followed by rapid snap-freezing of the heart and further tissue processing as described in 2.5. Metabolic flux through different pathways was measured using NMR-spectroscopy. During glycolysis, multiple enzymatic reactions convert glucose into pyruvate, which can be further processed to lactate by lactate dehydrogenase (LDH) (Fig. 4.3). Thus, if $\left[\mathrm{U}-{ }^{13} \mathrm{C}\right]$ glucose enters the cell and undergoes glycolysis, $\left[1,2,3-{ }^{13} \mathrm{C}\right]$ pyruvate arises and can be used as substrate to produce $\left[1,2,3-{ }^{13} \mathrm{C}\right]$ lactate, which is schematically illustrated in Fig. 4.4A. In the present study, $\left[1,2,3-{ }^{13} \mathrm{C}\right]$ lactate was used as readout for glycolytic activity. Compared to respective controls, cardiac glycolytic flux was significantly enhanced after two weeks of TAC (11.8 vs $9.4 \%$ enrichment of $\left[1,2,3-{ }^{13} \mathrm{C}\right]$ lactate, $\left.\mathrm{p}<0.05\right)$ suggesting more glycolysis in the heart following chronic pressure overload. The increase after two weeks of Shunt did not reach statistical significance (10.1 vs $8.8 \%$ enrichment of $\left[1,2,3-{ }^{13} \mathrm{C}\right]$ lactate, $\mathrm{p}=0.09$ ), but also indicates a tendency towards augmented glycolytic activity in the heart following chronic volume overload (Fig. 4.4B). 


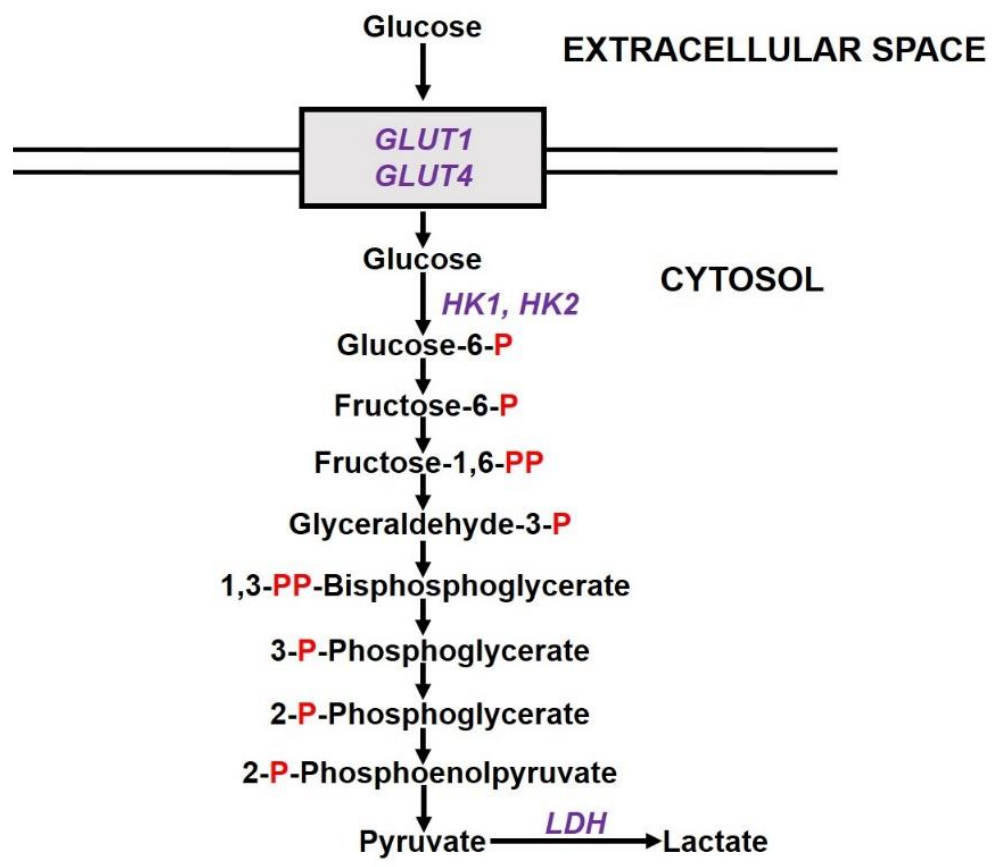

Figure 4.3: Schematic illustration of the glycolytic pathway. Glucose enters the cell through glucose transporters (GLUT1 and 4), followed by phosphorylation through hexokinases (HK1 and 2). Pyruvate is the product of a multistep reaction and can subsequently act as substrate for lactate dehydrogenase (LDH) to produce lactate. Relevant enzymes in the course of this study are shown in purple, a red P indicates a phosphate group.

A

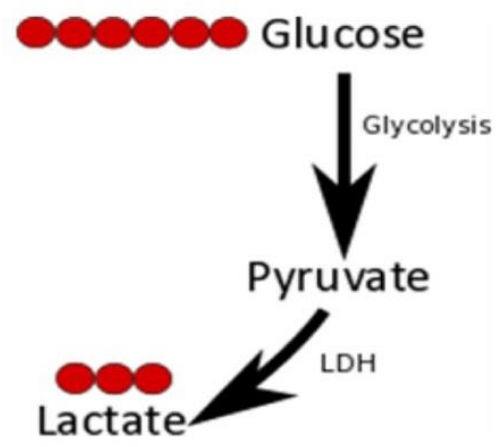

B ${ }^{13} \mathrm{C}$ Glycolysis Flux: $\left[1,2,3^{-13} \mathrm{C}\right]$ Lactate

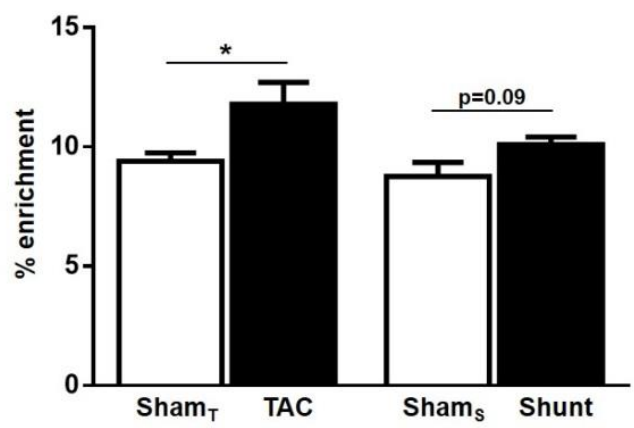

Figure 4.4: Glycolytic activity in murine hearts following two weeks of pressure and volume overload. Transverse aortic constriction (TAC) and Shunt surgery were performed in age-matched, male WT mice and compared to respective Sham controls $\left(\mathrm{Sham}_{\mathrm{T}}\right.$ for TAC, Sham $\mathrm{S}$ for Shunt). $\left[\mathrm{U}_{-}{ }^{13} \mathrm{C}\right]$ glucose was injected intraperitoneally followed by flux analysis using NMR-spectroscopy. $\left[1,2,3-{ }^{13} \mathrm{C}\right]$ lactate was used as readout for glycolysis, as depicted in A (LDH: Lactate Dehydrogenase; red circles indicate ${ }^{13} \mathrm{C}$-labelled carbon atoms). Mean data in $\mathbf{B}$ is shown as \% enrichment of $\left[1,2,3-{ }^{13} \mathrm{C}\right]$ lactate in murine hearts following two weeks of TAC and Shunt compared to respective controls. $n=4-5 /$ group; data are presented as mean $\pm \mathrm{SEM} ;{ }^{*} \mathrm{p}<0.05$ between TAC/Shunt and respective Sham controls using unpaired Student's t-test. 
Gene and protein expression of enzymes involved in glycolysis were measured after TAC and Shunt, and compared to respective controls. This was meant to give additional information about metabolic properties in the heart following pressure or volume overload. However, it should be noted that expression levels of enzymes alone are not very meaningful with respect to their activity. Lactate deyhrogenase A (LDHA) catalyzes the conversion of pyruvate to lactate, hexokinases 1 and 2 (HK1 and 2) the phosphorylation of glucose to glucose-6-phosphate (the first step in glycolysis) and glucose transporter 1 (GLUT1) is one of many transporters to bring glucose into the cell (Fig. 4.3). Gene expression of all these enzymes was significantly increased after TAC (LDHA: 1.4 fold, p<0.01; HK1: 2.7 fold, $\mathrm{p}<0.05$; HK2: 1.9 fold, $\mathrm{p}<0.05$ and GLUT1: 1.9 fold, $\mathrm{p}<0.05)$, whereas two weeks of volume overload only induced a significant upregulation of HK1-mRNA (1.4 fold, p<0.05) compared to respective controls (Fig. 4.5A-D). Thus, cardiac gene expression levels of glycolytic enzymes are in line with the previous findings of significantly increased glycolytic activity in the heart following pressure overload. This effect appears to be much less pronounced after volume overload. 
A

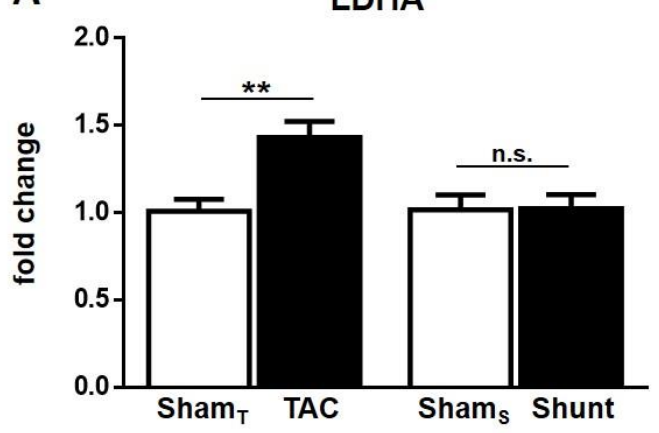

C

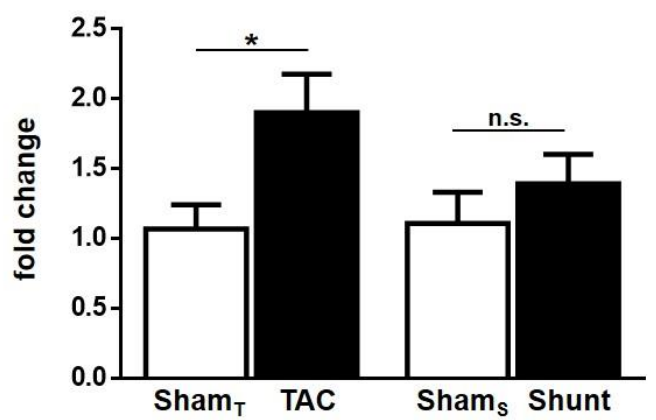

B

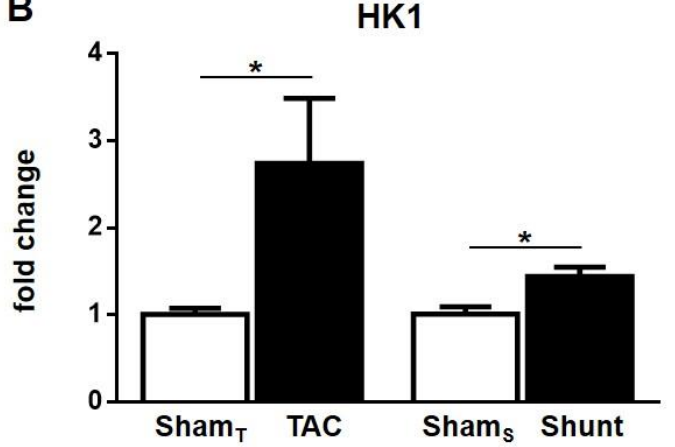

D

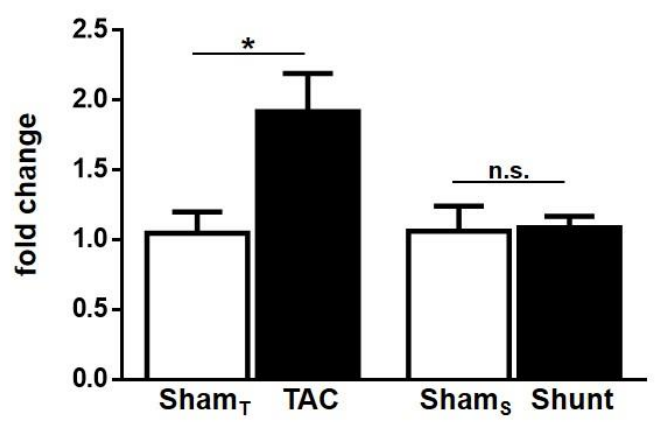

Figure 4.5: Cardiac gene expression of glycolytic enzymes in mice following two weeks of pressure and volume overload. Transverse aortic constriction (TAC) and Shunt surgery were performed in age-matched, male WT mice and compared to respective Sham controls (Sham ${ }_{\mathrm{T}}$ for TAC, Shams for Shunt). Cardiac mRNA levels of LDHA (lactate dehydrogenase A) (A), HK1 and 2 (hexokinases 1 and 2) (B,C), and GLUT1 (glucose transporter 1) (D) were measured two weeks post surgery using qRT-PCR. Values are presented as fold change compared to respective Sham controls, GAPDH was used for normalization. $\mathrm{n}=5$ /group; data are presented as mean $\pm \mathrm{SEM}$; $\mathrm{p}<0.05$, ** $\mathrm{p}<0.01$, n.s.: not significant between TAC/Shunt and respective Sham controls using unpaired Student's t-test.

Before analyzing cardiac protein expression levels of glycolytic enzymes, three commonly used loading controls for Western Blot analysis were tested with respect to their expression levels during cardiac hypertrophy. Fig. 4.6A reveals that both $\alpha$-tubulin as well as $\beta$-actin are induced in the heart after both two weeks of TAC and Shunt compared to respective controls. Cardiac protein levels of GAPDH (glyceraldehyde-3-phosphate dehydrogenase), however, seemed to be unchanged in response to both stresses. This is in line with Ponceau S-stainings reflecting similar protein loading between all different experimental groups (Fig. 4.6B,C). Thus, GAPDH was used as loading control in the course of this study. 
Following cardiac gene expression analysis, protein levels of LDHA, HK1 and GLUT1 after TAC, Shunt and respective Sham surgeries were assessed by Western Blot analysis. Representative images are shown in Fig. 4.7A. Similar to gene expression levels, LDHA and HK1 were only increased following pressure overload (LDHA: 1.4 fold, p=0.06; HK1: 1.5 fold, $\mathrm{p}<0.05$ ), but remained unchanged after Shunt compared to respective controls (Fig. 4.7A-C). GLUT1 expression, however, was significantly increased after Shunt (3.3 fold, $\mathrm{p}<0.05)$, but remained unchanged after two weeks of pressure overload compared to controls (Fig. 4.7A,D). This is contradictory to the previous observations on mRNA levels and therefore underlines the complexity of metabolic regulation in the heart.

A
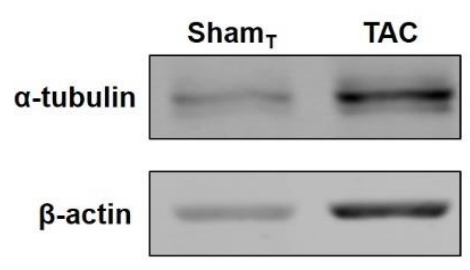

GAPDH
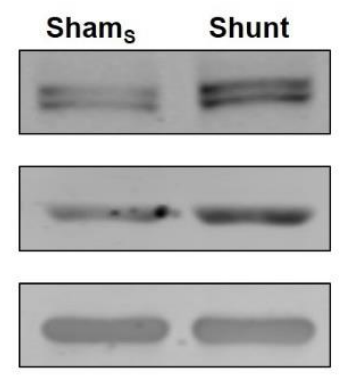

B

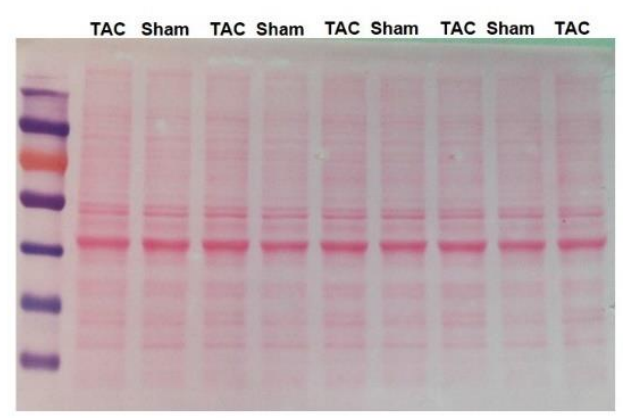

C

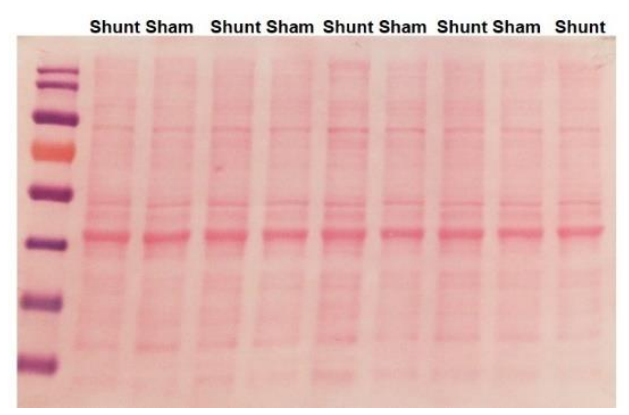

Figure 4.6: Evaluation of different loading controls for Western Blotting in murine heart lysates following two weeks of pressure and volume overload. Protein expression of $\alpha$-tubulin, $\beta$-actin and GAPDH, three commonly used loading controls for Western Blot analysis, were assessed in murine heart lysates two weeks after TAC, Shunt and their respective control surgery $\left(\mathrm{Sham}_{\mathrm{T}}\right.$ for TAC, Sham $\mathrm{S}$ for Shunt) by Western Blotting. Representative images are shown in A. Exemplary images for general protein staining from heart lysates using Ponceau $\mathrm{S}$ are shown in $\mathbf{B}$ (Sham ${ }_{\mathrm{T}}$ and TAC) and $\mathbf{C}\left(\right.$ Sham $_{\mathrm{S}}$ and Shunt). 
A
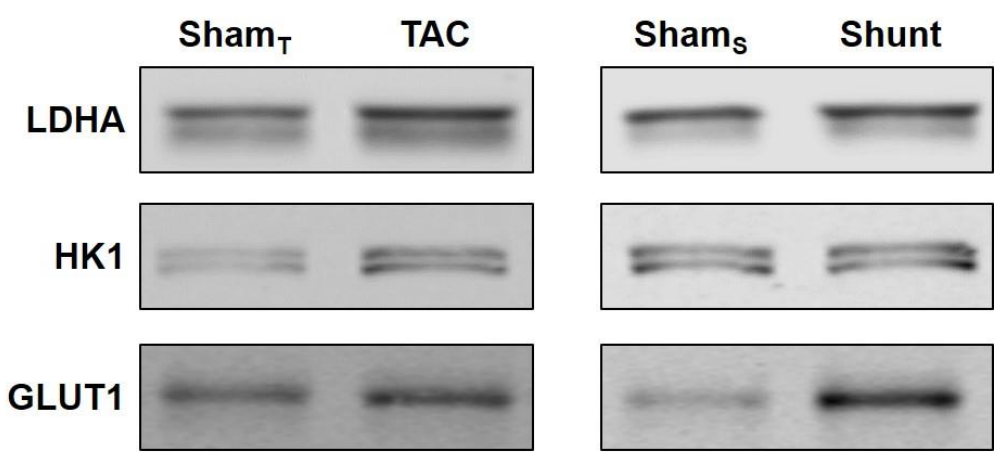

\section{GAPDH}
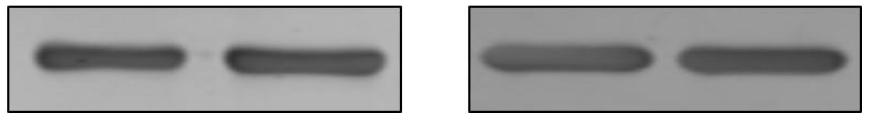

B

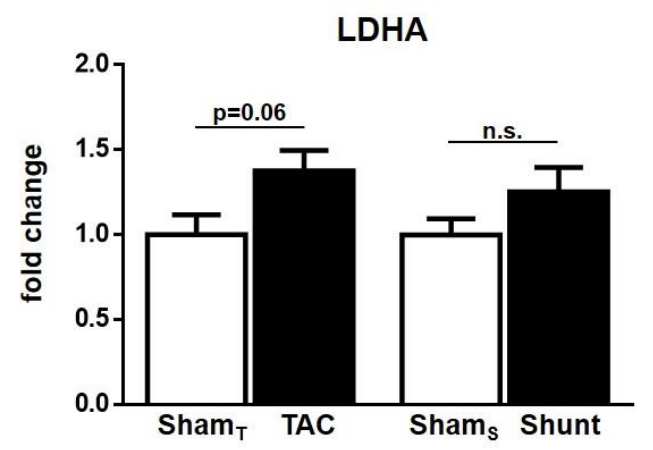

C

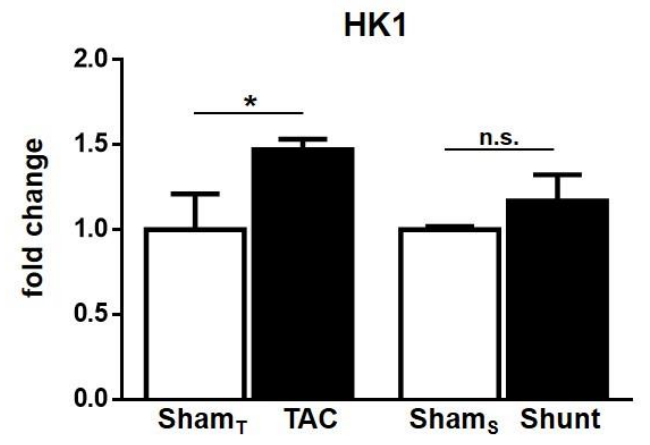

D

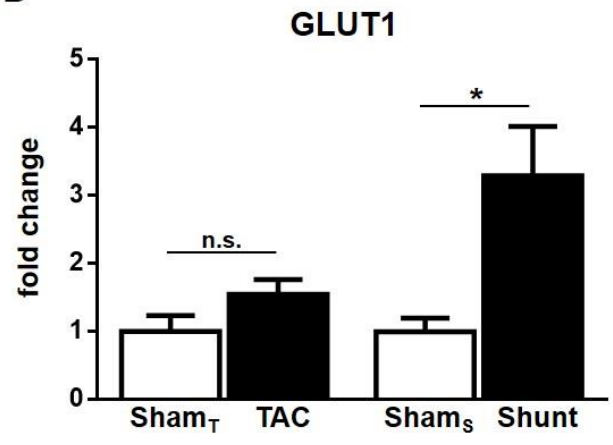

Figure 4.7: Cardiac protein expression of glycolytic enzymes in mice following two weeks of pressure and volume overload. Transverse aortic constriction (TAC) and Shunt surgery were performed in age-matched, male

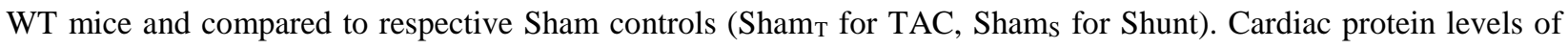
LDHA (lactate dehydrogenase A), HK1 (hexokinase 1) and GLUT1 (glucose transporter 1) were assessed two weeks post surgery by Western Blotting. Representative images are shown in A, densitometric quantification for each enzyme in B-D. Values are presented as fold change compared to respective Sham controls, GAPDH was used as loading control. $\mathrm{n}=4-5$ /group; data are presented as mean $\pm \mathrm{SEM} ;{ }^{*} \mathrm{p}<0.05$, n.s.: not significant between TAC/Shunt and respective Sham controls using unpaired Student's t-test. 


\subsubsection{TCA cycle}

Flux analysis using the novel in vivo ${ }^{13} \mathrm{C}$-labelling strategy in mice was performed as previously described. $\left[1,2,3-{ }^{13} \mathrm{C}\right]$ pyruvate arises from glycolysis of the administered $\left[\mathrm{U}-{ }^{13} \mathrm{C}\right]$ glucose and can enter the TCA cycle either via oxidative decarboxylation through PDH (pyruvate dehydrogenase) or through carboxylation via anaplerotic reactions catalyzed by PC (pyruvate carboxylase) or ME (malic enzyme). As the general scheme of TCA cycle metabolism in Fig. 4.8 illustrates, these anaplerotic reactions produce oxaloacetate and malate respectively, which can be converted into each other through malate dehydrogenase (MDH). Thus, labelled isotopomers arising from both anaplerotic reactions are identical. PDH activity and anaplerosis were separately assessed by using $\left[4,5-{ }^{13} \mathrm{C}\right]$ glutamate as a readout for flux through PDH and $\left[2,3-{ }^{13} \mathrm{C}\right]$ glutamate for anaplerosis. This is schematically illustrated in Fig. 4.9A. Compared to respective controls, metabolic flux through both PDH (8.2 vs $4.4 \%$ enrichment of $\left[4,5-{ }^{13} \mathrm{C}\right]$ glutamate, $\mathrm{p}<0.05)$ and anaplerotic reactions $\left(12.3\right.$ vs $7.0 \%$ enrichment of $\left[2,3-{ }^{13} \mathrm{C}\right]$ glutamate, $\left.\mathrm{p}<0.01\right)$ were significantly increased after two weeks of TAC suggesting enhanced TCA cycle activity in the heart following pressure overload. Such changes were not observed after two weeks of volume overload (Fig. 4.9B,C) - a similar finding to the lack of major changes in glycolytic activity after Shunt.

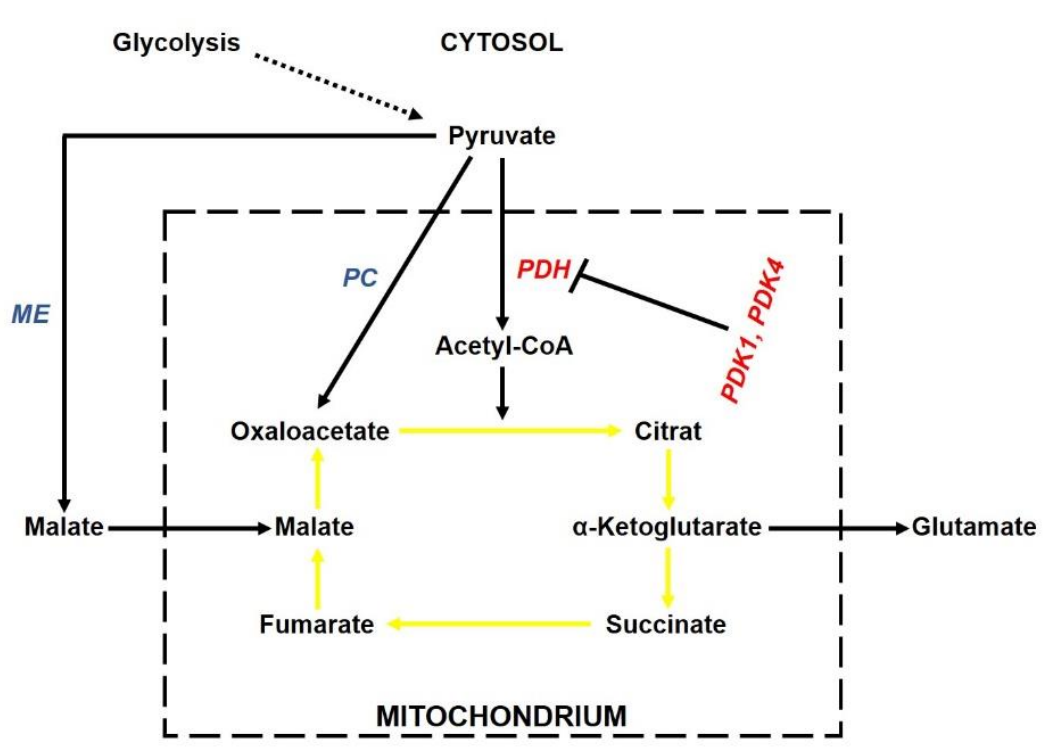

Figure 4.8: Schematic illustration of TCA cycle metabolism. Glycolysis-derived pyruvate can enter the mitchondrium, followed by conversion to acetyl-CoA through pyruvate dehydrogenase (PDH) and entry into the TCA (tricarboxylic acid) cycle. PDH activity can be inhibited through phosphorylation by pyruvate dehydrogenase kinases (PDK1 and 4). Pyruvate can also act as substrate for anaplerotic reactions such as production of oxaloacetate 


\section{Results II}

and malate through pyruvate carboxylase (PC) and malic enzyme (ME) respectively. Relevant enzymes in the course of this study are shown in red (PDH activity) or blue (anaplerosis), yellow arrows indicate the TCA cycle.

A

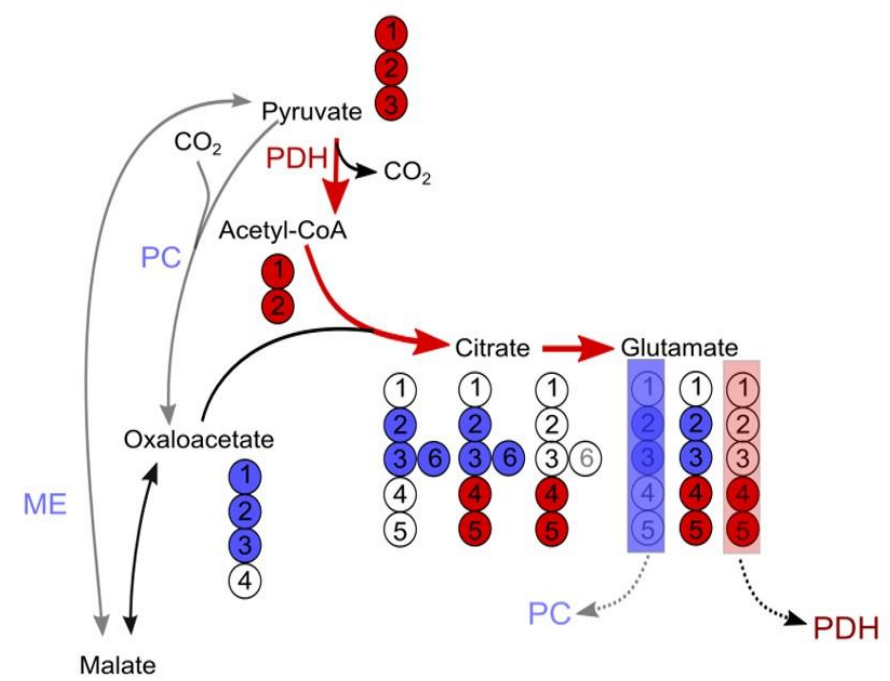

B

${ }^{13} \mathrm{C}$ PDH Flux:

$\left[4,5-{ }^{13} \mathrm{C}\right]$ Glutamate

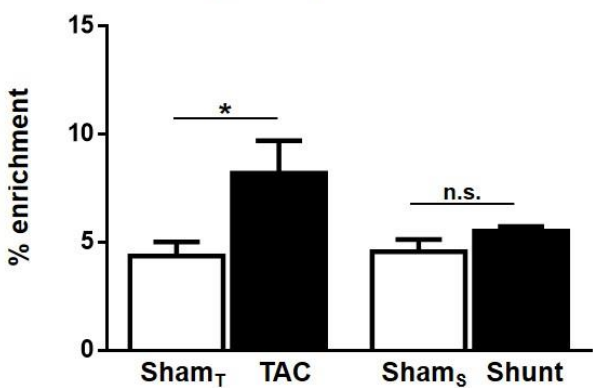

C 13C PC Flux: $\left[2,3-{ }^{13} \mathrm{C}\right]$ Glutamate

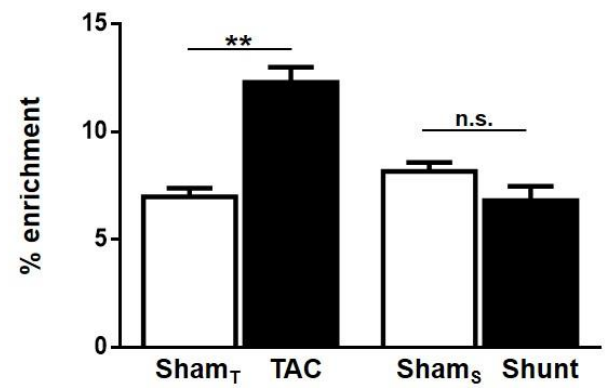

Figure 4.9: TCA cycle activity in murine hearts following two weeks of pressure and volume overload. Transverse aortic constriction (TAC) and Shunt surgery were performed in age-matched, male WT mice and compared to respective Sham controls $\left(\mathrm{Sham}_{\mathrm{T}}\right.$ for TAC, Sham $\mathrm{S}$ for Shunt). $\left[\mathrm{U}_{-}{ }^{13} \mathrm{C}\right]$ glucose was injected intraperitoneally followed by flux analysis using NMR-spectroscopy. $\left[4,5-{ }^{13} \mathrm{C}\right]$ glutamate was used as readout for pyruvate dehydrogenase (PDH) activity, $\left[2,3-{ }^{13} \mathrm{C}\right]$ glutamate for anaplerosis mediated by pyruvate carboxylase (PC) or malic enzyme (ME). A illustrates this schematically: $\left[1,2,3-{ }^{13} \mathrm{C}\right]$ pyruvate is produced through glycolysis of [U$\left.{ }^{13} \mathrm{C}\right]$ glucose and can subsequently enter the TCA cycle either through PDH or anaplerosis, catalyzed by PC or ME. Labelled carbons of isotopomers arising from PDH are shown in dark red, from anaplerosis in dark blue; light red and light blue rectangles indicate the two isotopomers used as readouts for PDH and PC activity respectively. Mean data are shown as \% enrichment of $\left[4,5-{ }^{13} \mathrm{C}\right]$ glutamate $(\mathbf{B})$ and $\left[2,3-{ }^{13} \mathrm{C}\right]$ glutamate $(\mathbf{C})$ in murine hearts two weeks post surgery compared to respective Sham controls. $\mathrm{n}=4-5 /$ group; data are presented as mean \pm SEM; $* \mathrm{p}<0.05$, ** p <0.01, n.s.:not significant between TAC/Shunt and respective Sham controls using unpaired Student's t-test.

Gene and protein expression of enzymes involved in TCA cycle metabolism were measured after TAC, Shunt and compared to respective controls. Pyruvate dehydrogenase-alpha 1 (PDHA1) is a 
component of the PDH multienzyme complex catalyzing the reaction from pyruvate to acetylCoA. Pyruvate dehydrogenase kinases 1 and 4 (PDK1 and 4) phosphorylate PDH and thereby inactive it. Pyruvate carboxylase (PC) catalyzes the addition of $\mathrm{CO}_{2}$ to pyruvate to form oxaloacetate, an important step in anaplerosis. The significant increase in gene expression of PDHA1 after TAC (2.1 fold, $\mathrm{p}<0.01)$ is consistent with the previously reported increase in PDH activity, which is not evident after two weeks of Shunt compared to respective controls (Fig. 4.10A). Cardiac PDK1 expression levels, however, show similar increaes under both stress conditions (Fig. 4.10B), whereas PDK4 is decreased (Fig. 4.10C). In the case of volume overload stress, this decrease even reaches statistical significance $(0.52$ fold, $\mathrm{p}<0.05)$.

A

PDHA1

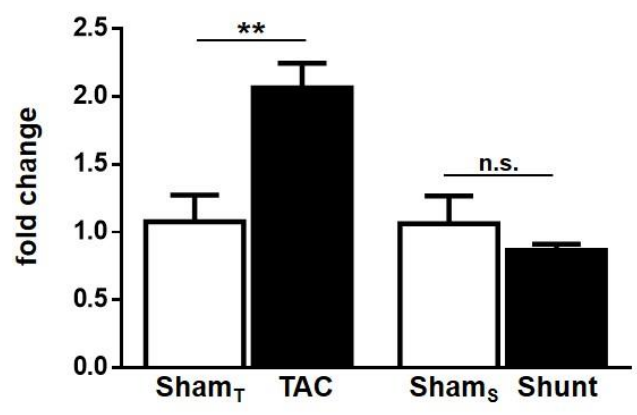

B

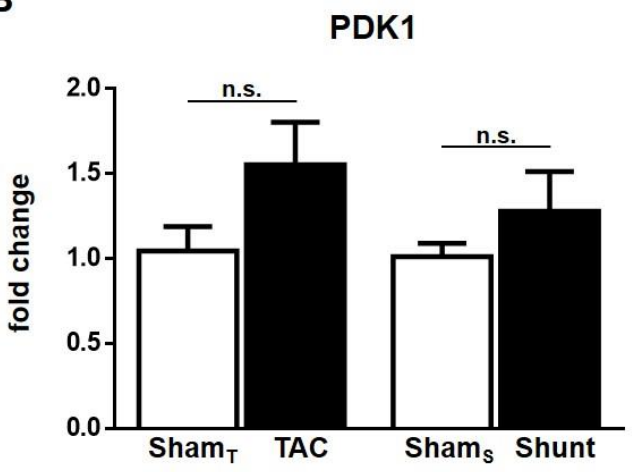

PDK4

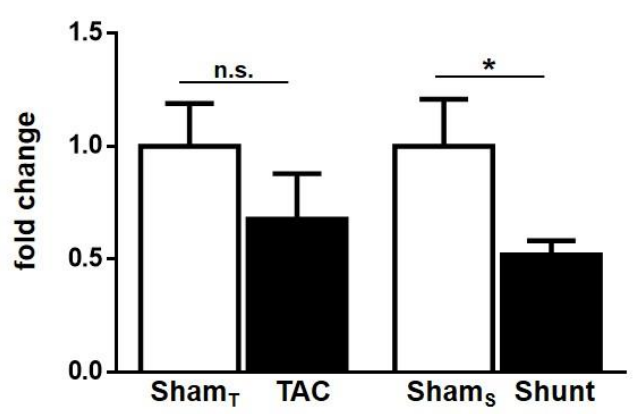

Figure 4.10: Cardiac gene expression of enzymes related to TCA cycle metabolism in mice following two weeks of pressure and volume overload. Transverse aortic constriction (TAC) and Shunt surgery were performed in age-matched, male WT mice and compared to respective Sham controls (Sham $\mathrm{T}$ for TAC, Sham $\mathrm{S}$ for Shunt). Cardiac mRNA levels of PDHA1 (pyruvate dehydrogenase-alpha 1) (A), PDK1 and 4 (pyruvate dehydrogenase kinases 1 and 4) (B,C) were measured two weeks post surgery using qRT-PCR. Values are presented as fold change compared to respective Sham controls, GAPDH was used for normalization. $\mathrm{n}=5$ /group; data are presented as mean \pm SEM; $* \mathrm{p}<0.05, * * \mathrm{p}<0.01$, n.s.: not significant between TAC/Shunt and respective Sham controls using unpaired Student's t-test. 
Cardiac protein expression levels of the PDH component E1-subunit- $\alpha$ (PDH E1- $\alpha$ ), PDK1, PDK4 and PC after TAC, Shunt and respective Sham surgeries were measured using Western Blot analysis. Representative images are shown in Fig. 4.11A. Despite the observed changes in TCA cycle activity and gene expression of enzymes involved in TCA cycle metabolism, protein expression of all enzymes was similar throughout all four experimental groups (Fig. 4.11A-E). It again highlights how challenging and diverse metabolic assessment in the heart can be, especially with respect to enzyme expression analysis. 
A

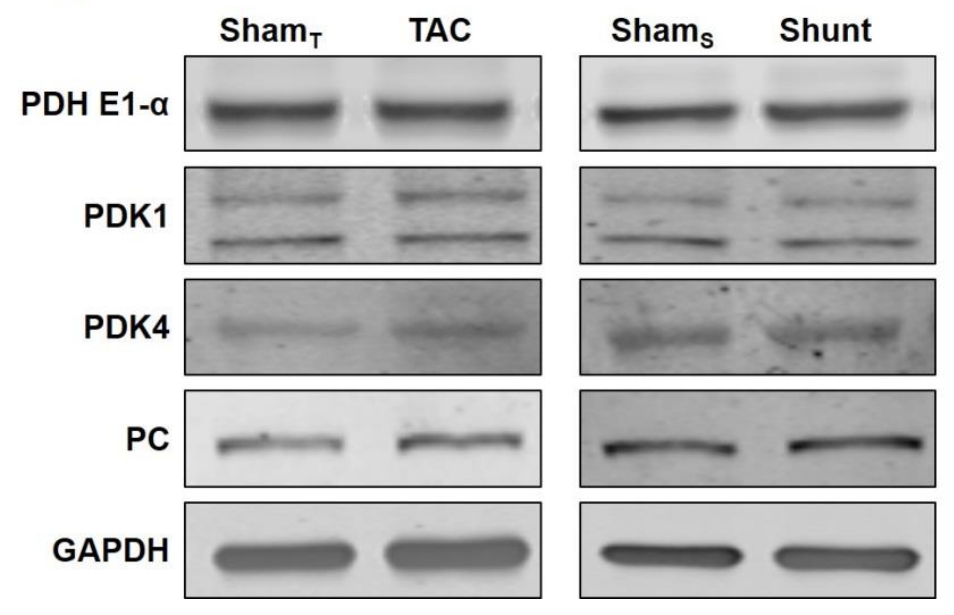

B

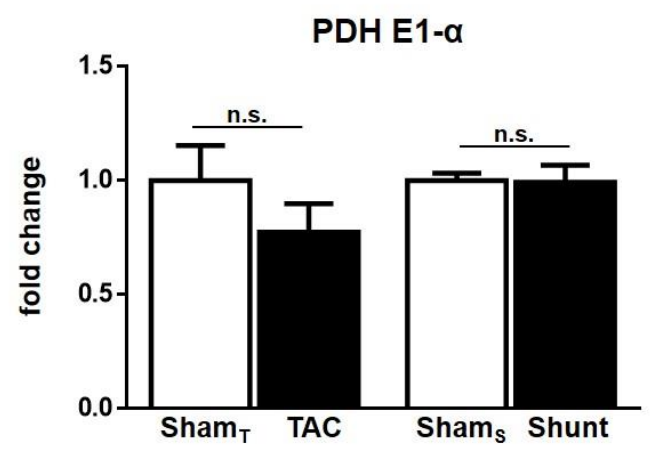

D

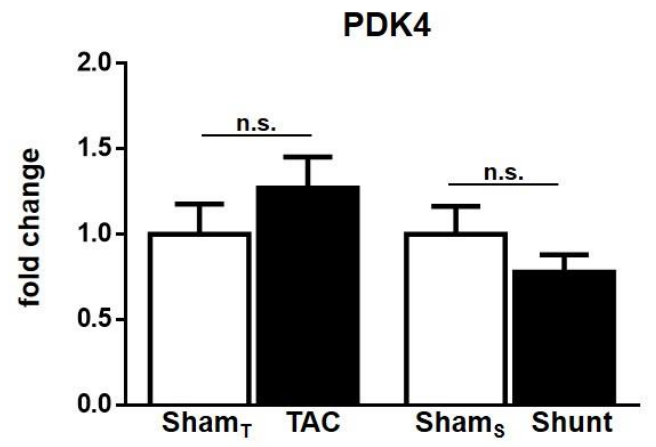

C

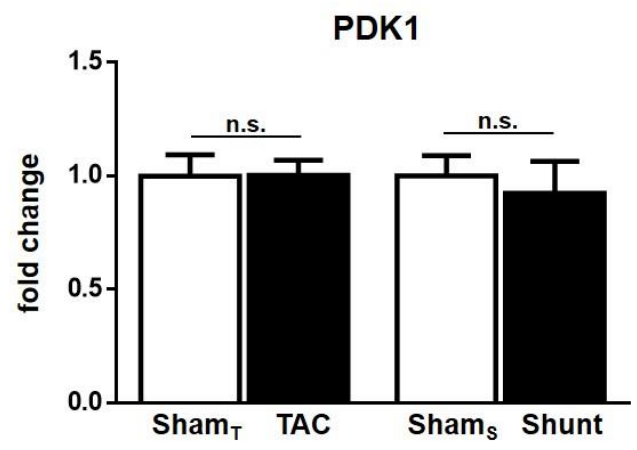

E

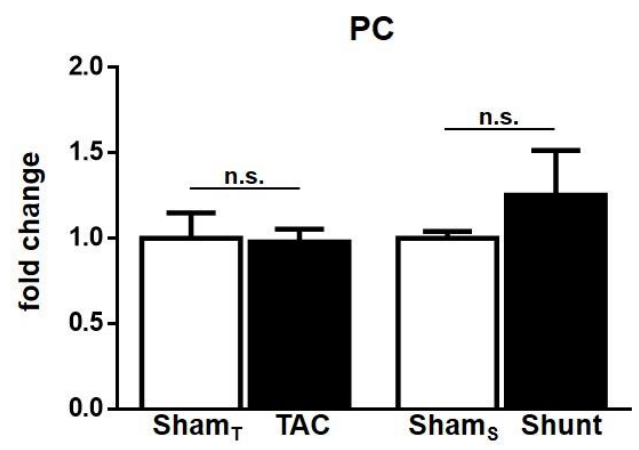

Figure 4.11: Cardiac protein expression of enzymes related to TCA cycle metabolism in mice following two weeks of pressure and volume overload. Transverse aortic constriction (TAC) and Shunt surgery were performed in age-matched, male WT mice and compared to respective Sham controls (Sham $\mathrm{T}$ for TAC, Sham $\mathrm{S}$ for Shunt). Cardiac protein levels of PDH E1- $\alpha$ (pyruvate dehydrogenase E1-subunit- $\alpha$ ), PDK1 and 4 (pyruvate dehydrogenase kinases 1 and 4) and PC (pyruvate carboxylase) were assessed two weeks post surgery via Western Blotting. Representative images are shown in $\mathbf{A}$, densitometric quantification for each enzyme in B-E. Values are presented as fold change compared to respective Sham controls, GAPDH was used as loading control. n=4-5/group; data are 
presented as mean \pm SEM; n.s.: not significant between TAC/Shunt and respective Sham controls using unpaired Student's t-test.

\section{$\underline{\text { 4.3.3 Glutamine metabolism }}$}

The novel in vivo ${ }^{13} \mathrm{C}$-labelling strategy with subsequent flux analysis using NMR-spectroscopy was used to assess glutamine synthesis in murine hearts following TAC, Shunt and respective Sham controls. Glutamine is an important substrate for the hexosamine biosynthetic pathway (HBP) and glutathione biosynthesis. Both pathways are implicated in the development of cardiovscular diseases ${ }^{131-134}$ and were therefore of particular interest. As described previously (Section 4.3.2), administered $\left[\mathrm{U}_{-}{ }^{13} \mathrm{C}\right]$ glucose can be metabolized to $\left[4,5-{ }^{13} \mathrm{C}\right]$ or $\left[2,3-{ }^{13} \mathrm{C}\right]$ glutamate (or $\left[2,3,4,5-{ }^{13} \mathrm{C}\right]$ glutamate, which is not of particular interest in this study), depending on whether it is formed through PDH or anaplerotic reactions. This glutamate can be further converted to glutamine through glutamine synthetase (GS). As illustrated in Fig. 4.12A, $\left[4,5-{ }^{13} \mathrm{C}\right]$ glutamine was used as readout for glutamine synthetase activity. Compared to respective controls, metabolic flux through PDH into glutamine was significantly enhanced after two weeks of TAC (3.0 vs $1.4 \%$ enrichment of $\left[4,5-{ }^{13} \mathrm{C}\right]$ glutamine, $\left.\mathrm{p}<0.01\right)$ suggesting increased glutamine synthesis in the heart following chronic pressure overload. As for glycolytic and TCA cycle activity, two weeks of volume overload did not change glucose-dependent glutamine synthesis in the heart significantly (Fig. 4.12B).

A

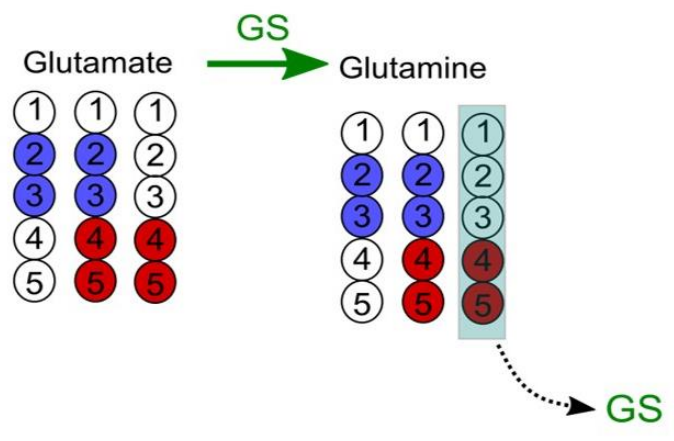

B

13C GS Flux:

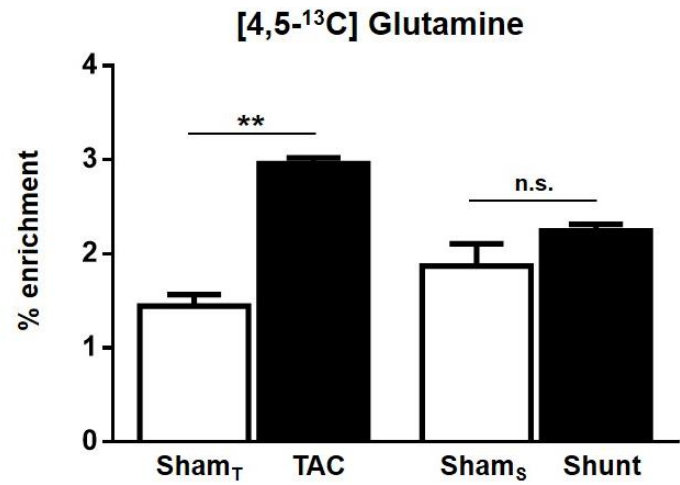

Figure 4.12: Glutamine biosynthesis in murine hearts following two weeks of pressure and volume overload. Transverse aortic constriction (TAC) and Shunt surgery were performed in age-matched, male WT mice and compared to respective Sham controls $\left(\mathrm{Sham}_{\mathrm{T}}\right.$ for TAC, Shams for Shunt). $\left[\mathrm{U}_{-}{ }^{13} \mathrm{C}\right]$ glucose was injected intraperitoneally followed by flux analysis using NMR-spectroscopy. $\left[4,5-{ }^{13} \mathrm{C}\right]$ glutamine was used as readout for glutamine synthesis, which is schematically illustrated in A: TCA cycle-derived glutamate is converted to glutamine 
through glutamine synthetase (GS). Labelled carbons of isotopomers arising from PDH are shown in red, from anaplerosis in blue. Mean data in $\mathbf{B}$ is shown as \% enrichment of $\left[4,5-{ }^{13} \mathrm{C}\right]$ glutamine in murine hearts two weeks post surgery. $\mathrm{n}=4-5 /$ group; data are presented as mean $\pm \mathrm{SEM}$; ** $\mathrm{p}<0.01$, n.s.: not significant between TAC/Shunt and respective Sham controls using unpaired Student's t-test.

As schematically depicted in Fig 4.13 and previously mentioned, glutamine can be a substrate in the hexosamine biosynthetic pathway (HBP). The rate limiting step of this pathway is the prduction of glucosamine-6-phosphate from fructose-6-phosphate and glutamine, a reaction catalzyed by GFAT 1 or 2 (glutamine:fructose-6-phosphate aminotransferase 1 or 2). Glucosamine-6-phosphate is used for the production of UDP-GlcNAc (uridine diphosphate Nacetylglucosamine), the final substrate for O-GlcNAcylation modification of proteins. In the heart, this protein modification has been linked with both cardioprotection as well as disease $\operatorname{progression}^{135}$.

Glutamine can also serve as substrate for glutathione biosynthesis (Fig. 4.13 upper part). As previously mentioned, glutamate is converted through glutamine synthetase (GS) to produce glutamine. However, glutaminase (GLS) catalyzes this reaction in the opposite direction, thereby producing glutamate from glutamine. In a two-step reaction, cysteine and glycine are added to glutamate to form glutathione, one of the main intracellular redox buffers.

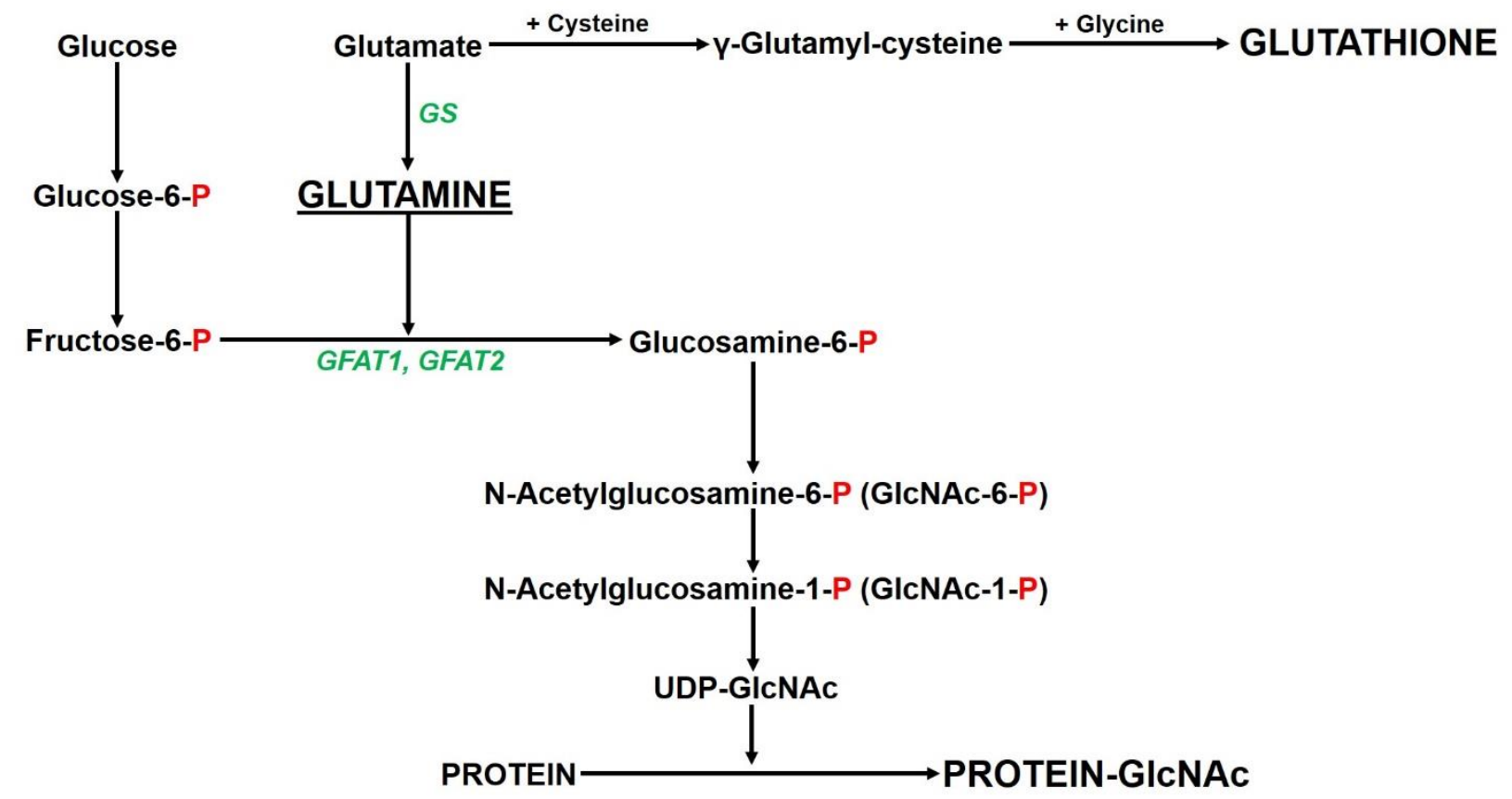

Figure 4.13: Schematic illustration of glutamine-involvment in the hexosamine biosynthetic pathway (HBP) and glutathione synthesis. HBP: Glutamate is converted to glutamine through glutamine synthetase (GS). GFAT 1 


\section{Results II}

and 2 (glutamine:fructose-6-phosphate aminotransferases 1 and 2) use glutamine and fructose-6-phosphate as substrates to produce glucosamine-6-phosphate, which is converted via multiple steps to uridine diphosphate Nacetylglucosamine (UDP-GlcNAc), the final substrate for O-GlcNAc modification of proteins. Glutamine can also be converted back to glutamate through glutaminase (GLS). Glutamate can then be used as precursor for glutathione biosynthesis. Relevant enzymes in the course of this study are shown in green, a red P indicates a phosphate group.

The HBP was assessed following two weeks of TAC, Shunt and respective Sham controls by cardiac protein expression measurements of HBP-related proteins by Western Blotting. Representative images are shown in Fig. 4.14A. Expression levels of GS (1.6 fold, p<0.05) and GFAT2 (1.6 fold, p<0.01) were significantly increased after two weeks of pressure overload compared to controls (Fig. 4.14A,B,D), which was in line with significant increases in OGlcNAcylation modification of proteins (1.6 fold, p<0.05) (Fig. 4.14A,E). Interestingly, GFAT1 levels did not change through pressure overload (Fig. 4.14A,C) suggesting a specific GFAT2dependent mechanism. Protein levels of GS, GFAT1, GFAT2 and O-GlcNAcylation of proteins remained unchanged after two weeks of volume overload (Fig. 4.14A-E). Thus, the increased glutamine production in response to chronic pressure overload might be related to the higher demand for HBP activity resulting in O-GlcNAcylation modification of proteins. After two weeks of volume overload, this does not seem to be of relevance.

In order to assess potential changes in glutathione biosynthesis following pressure and volume overload, cardiac protein expression levels of glutaminase (GLS), which catalyzes the conversion of glutamine to glutamate, were assessed. Indeed, two weeks of pressure overload caused a significant increase in GLS levels (1.4 fold, $\mathrm{p}<0.05)$, which was not detected after chronic volume overload compared to respective controls (Fig. 4.15A,B). Additionally, the ratio of reduced versus oxidized glutathione, measured with the GSH-Glo ${ }^{\mathrm{TM}}$ Assay Kit (Promega), was significantly reduced after two weeks of TAC $(0.7$ fold, $\mathrm{p}<0.05$; indicating a more oxidized environment), but not Shunt (1.1 fold, p=n.s.) compared to respective controls (Fig. 4.15C). Thus, more glutathione is presumably needed in order to buffer increased oxidative stress following chronic pressure overload in the heart. After two weeks of volume overload, this does not seem to be necessary due to a well-compensated redox environment.

The need for increased HBP activity and glutathione biosynthesis following pressure, but not volume overload, might therefore explain the increased glutamine synthesis after TAC. 
A

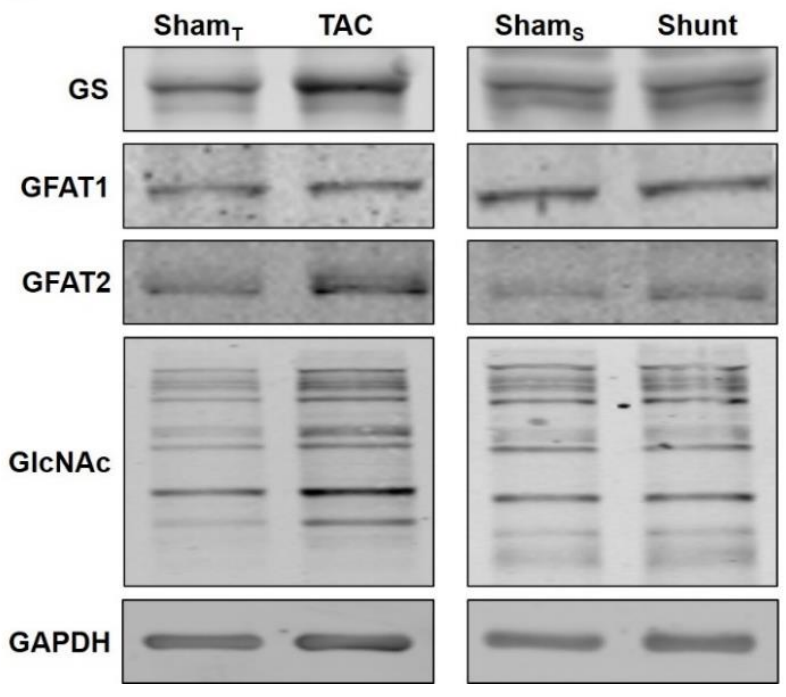

B GS

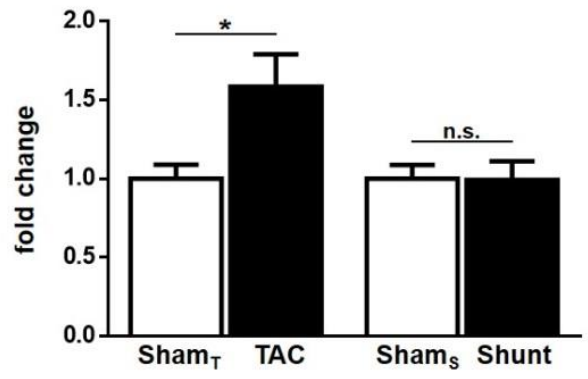

D

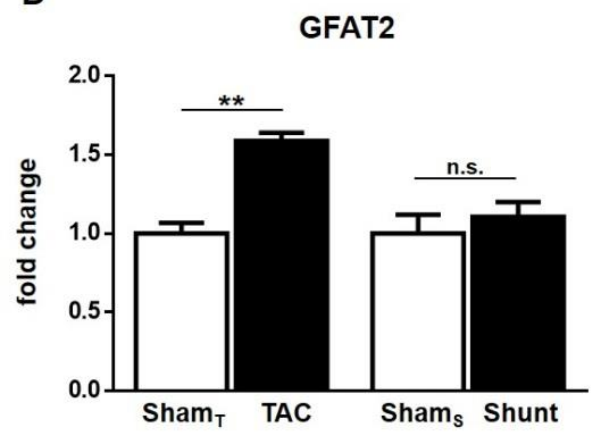

C GFAT1

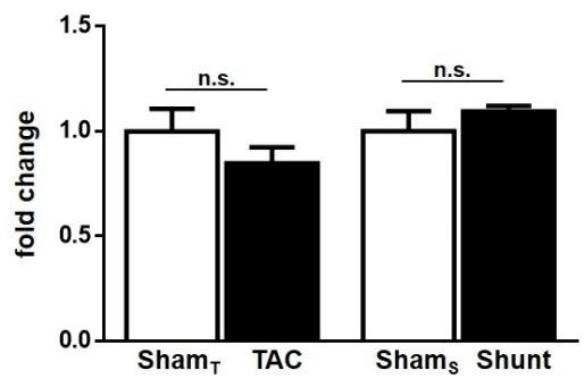

E

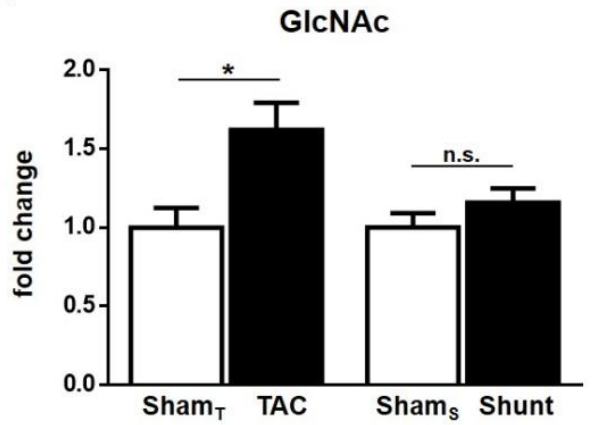

Figure 4.14: Cardiac protein expression of enzymes related to glutamine metabolism in mice following two weeks of pressure and volume overload. Transverse aortic constriction (TAC) and Shunt surgery were performed in age-matched, male WT mice and compared to respective Sham controls (Sham $\mathrm{T}$ for TAC, Sham $\mathrm{S}$ for Shunt). Cardiac protein levels of GS (glutamine synthetase), GFAT1 and 2 (glutamine:fructose-6-phosphate aminotransferases 1 and 2), and GlcNAc (O-linked-N-acetylglucosamine) were assessed two weeks post surgery via Western Blotting. Representative images are shown in A, densitometric quantification in B-E. Values are presented 
as fold change compared to respective Sham controls, GAPDH was used as loading control. n=4-5/group; data are presented as mean $\pm \mathrm{SEM}$; $\mathrm{p}_{\mathrm{p}}<0.05$, ** $\mathrm{p}<0.01$, n.s.: not significant between TAC/Shunt and respective Sham controls using unpaired Student's t-test.

A

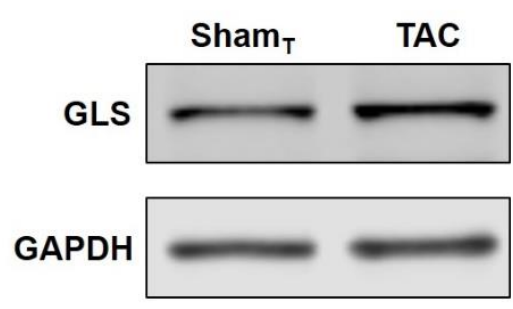

C
B
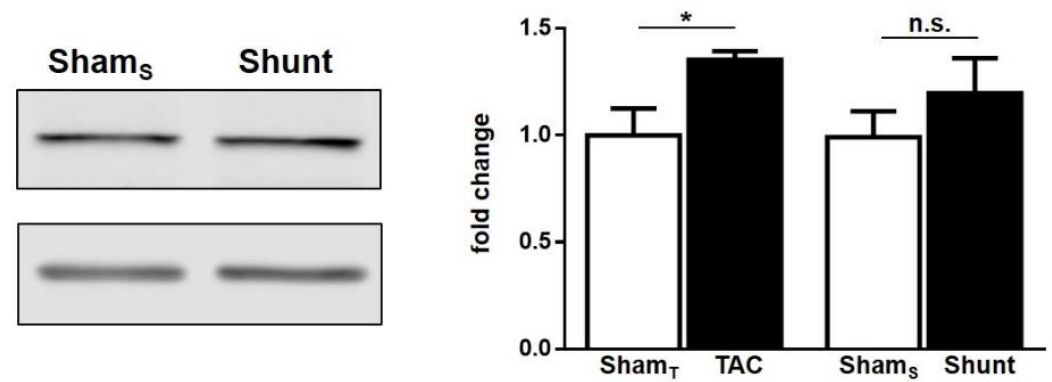

Reduced/oxidized Glutathione

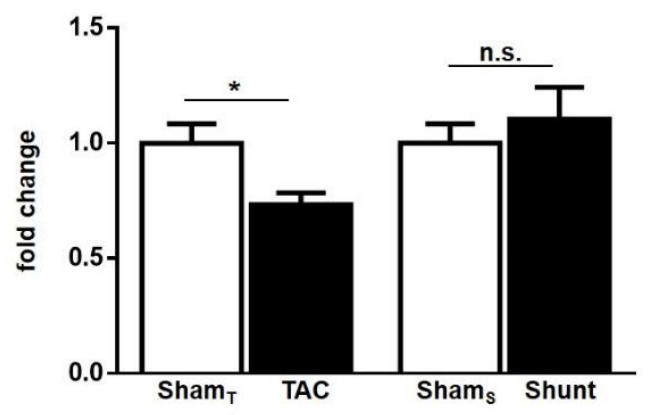

Figure 4.15: Glutamine-involvement in cardiac glutathione biosynthesis following two weeks of pressure and volume overload. Transverse aortic constriction (TAC) and Shunt surgery were performed in age-matched, male WT mice and compared to respective Sham controls (Sham $\mathrm{T}_{\mathrm{T}}$ for TAC, Shams for Shunt). For glutathione biosynthesis, glutamine needs to be converted to glutamate, a reaction catalyzed by glutaminase (GLS). Glutaminase protein expression two weeks post surgery was evaluated by Western Blotting. Representative images are shown in A, densitometric quantification in $\mathbf{B}$, GAPDH was used as loading control. Reduced versus oxidized glutathione ratios were assessed in murine heart lysates two weeks post surgery using the GSH-Glo ${ }^{\mathrm{TM}}$ Assay (Promega) (C). Values in $\mathbf{B}$ and $\mathbf{C}$ are presented as fold change compared to respective Sham controls. $n=4-9$ /group; data are presented as mean $\pm \mathrm{SEM} ; * \mathrm{p}<0.05$, n.s.: not significant between TAC/Shunt and respective Sham controls using unpaired Student's t-test.

\subsubsection{Gene expression of enzymes related to fatty acid oxidation (FAO) and fatty acid synthesis} $\underline{(\boldsymbol{F A S})}$

Fatty acid oxidation (FAO) and fatty acid synthesis (FAS) were analyzed in the heart following two weeks of pressure and volume overload by measuring gene expression levels of involved enzymes. As depicted in Fig. 4.16, carnitine palmitoyltransferases 1 and 2 (CPT1 and 2) are 
linked to transport of fatty acids into mitochondria and are therefore involved in FAO. Expression levels of these enzymes remained unchanged through both TAC and Shunt compared to controls (Fig. 4.17A,B). Acetyl-CoA carboxylases $\alpha$ and $\beta$ (ACACA and ACACB), and fatty acid synthase (FASN) are related to FAS (Fig. 4.16). Both ACACA (1.8 fold, p<0.01) and FASN (1.7 fold, $\mathrm{p}<0.05)$ displayed significant increases on mRNA level following chronic pressure overload compared to controls, which was not evident after chronic volume overload (Fig. 4.17C,E). ACACB-mRNA levels remained unchanged in all four experimental groups (Fig. 4.17D).

Taken together, with respect to cardiac gene expression profiles, there seems to be a shift towards increased FAS without alterations in FAO after chronic pressure overload. After chronic volume overload, both FAO and FAS appear to be unchanged, again implying a more complex metabolic response in response to chronic pressure compared to volume overload.

\section{CYTOSOL}

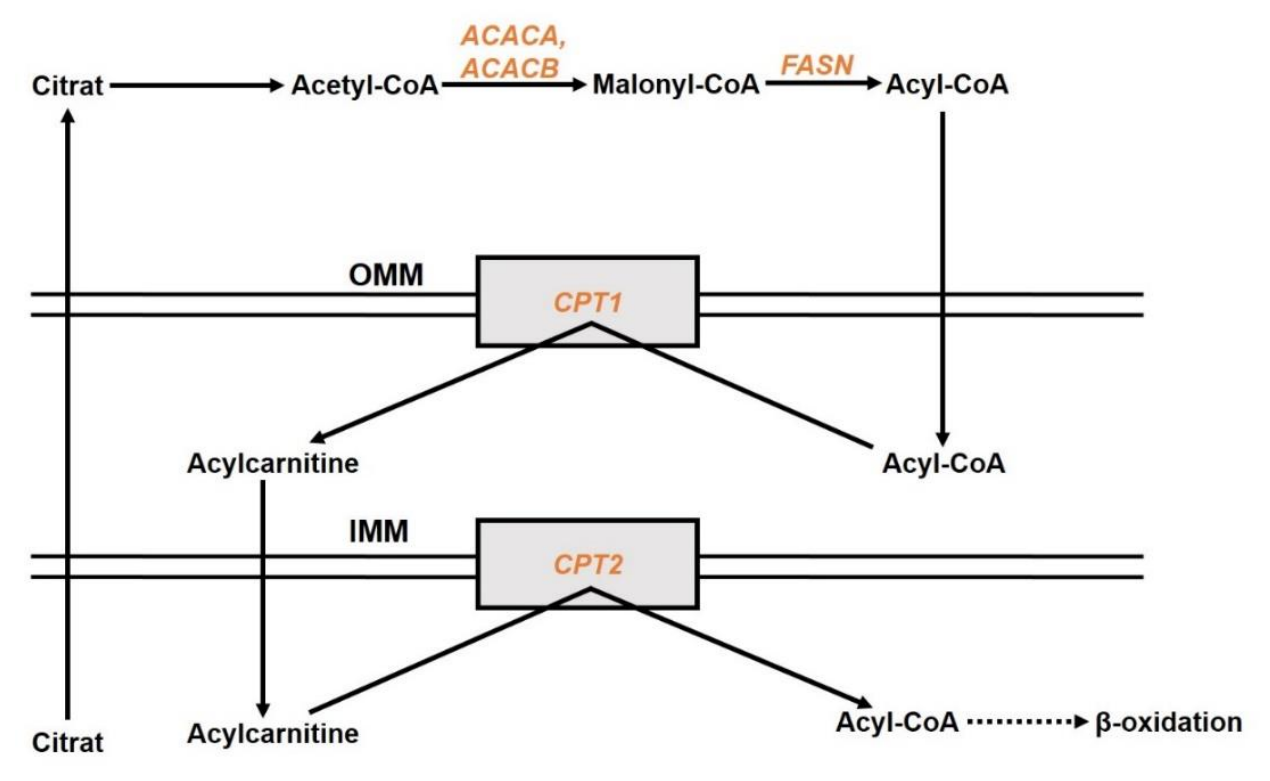

MITOCHONDRIUM

Figure 4.16: Schematic illustration of fatty acid synthesis (FAS) and oxidation (FAO). Citrat is transported from the mitchondrium into the cytosol, where it can be converted into acetyl-CoA. Acetyl-CoA can then act as substrate for acetyl-CoA carboxylases $\alpha$ and $\beta$ (ACACA and ACACB) to produce malonyl-CoA, the key substrate for fatty acid synthase (FASN)-mediated production of acyl-CoA (long-chain fatty acid-CoA). Acyl-CoA enters the outer mitchonchondrial membrane (OMM), but in order for it to enter the inner mitochondrial membrane (IMM), carnitine needs to be added via carnitine palmitoyltransferase 1 (CPT1) to produce Acylcarnitine. Acylcarnitine can then cross 


\section{Results II}

the IMM. In the mitochondrium, carnitine palmitoyltransferase 2 (CPT) catalyzes the regeneration of acyl-CoA, which can then be used for $\beta$-oxidation. Relevant enzymes in the course of this study are shown in brown.

A

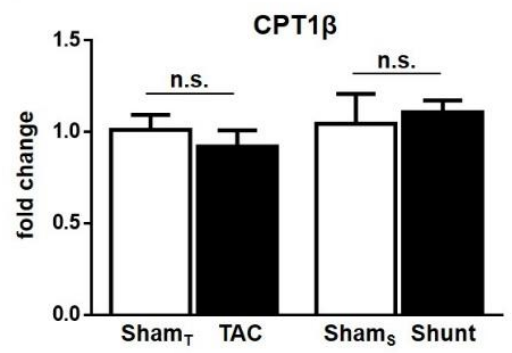

C

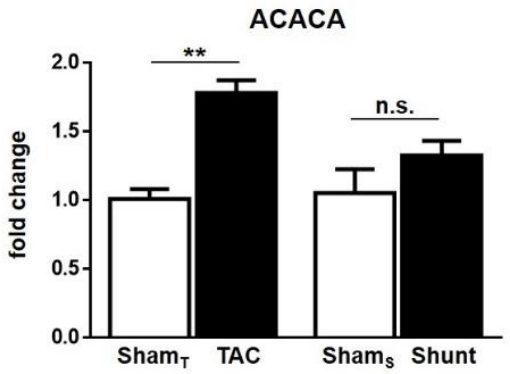

B

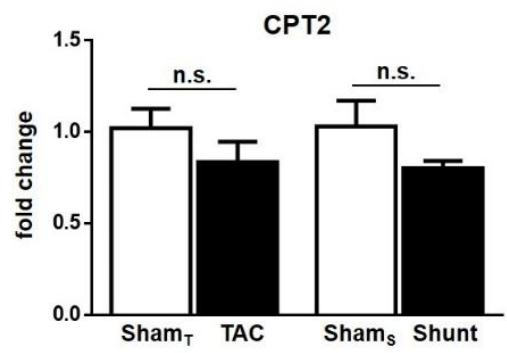

D

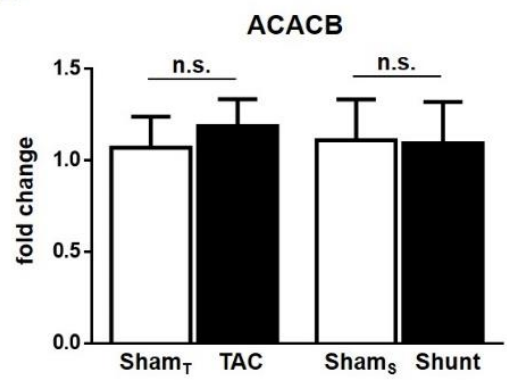

E

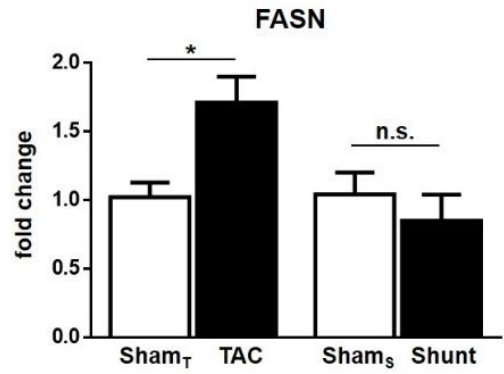

Figure 4.17: Cardiac gene expression of enzymes related to fatty acid oxidation (FAO) and synthesis (FAS) in mice following two weeks of pressure and volume overload. Transverse aortic constriction (TAC) and Shunt surgery were performed in age-matched, male WT mice and compared to respective Sham controls $\left(\mathrm{Sham}_{\mathrm{T}}\right.$ for TAC, Shams for Shunt). Cardiac mRNA levels of CPT1 $\beta$ and 2 (carnitine palmitoyltransferases $1 \beta$ and 2 ) as readouts for FAO (A,B), ACACA and ACACB (acetyl-CoA carboxylases $\alpha$ and $\beta$ ), and FASN (fatty acid synthase) for FAS (CE) were measured two weeks post surgery using qRT-PCR. Values are presented as fold change compared to respective Sham controls, GAPDH was used for normalization. $n=5$ /group; data are presented as mean \pm SEM; * $\mathrm{p}<0.05, * * \mathrm{p}<0.01$, n.s.: not significant between TAC/Shunt and respective Sham controls using unpaired Student's t-test. 


\subsection{Discussion}

As expected, two weeks of transverse aortic constriction (TAC) and aortocaval fistula (Shunt) in mice are associated with different cardiac remodelling patterns. Whereas TAC, as a pressure overload model, causes concentric remodelling with increases in wall thickness, Shunt induces eccentric remodelling, characterized by left ventricular dilation (Fig. 4.1, Table 4.1). Despite the same amount of left ventricular hypertrophy in both haemodynamic stress models, systolic dysfunction only occurred in response to pressure overload (Fig. 4.2, Table 4.1). Hence, a certain level of concentric hypertrophy appears to be associated with a more detrimental phenotype in the heart compared to a similar level of eccentric hypertrophy. This is in line with a previous study showing cardiac dysfunction and interstitial fibrosis in mice subjected to chronic TAC, but not Shunt ${ }^{27}$. Chronic pressure overload was furthermore associated with higher mortality in these mice compared to chronic volume overload. However, the increase in left ventricular hypertrophy and mean total wall stress in both models were similar. Thus, different signalling is likely to be involved in the cardiac responses to these haemodynamic stresses, which might account for the different phenotypes. A particular focus in the course of this study was on the potential difference in cardiac metabolism following pressure and volume overload.

Using a novel in vivo ${ }^{13} \mathrm{C}$-labelling strategy through $\left[\mathrm{U}-{ }^{13} \mathrm{C}\right]$ glucose administration in mice subjected to either TAC or Shunt, glucose metabolism in the heart was assessed by flux analysis using NMR-spectroscopy. In response to chronic pressure overload, glycolytic activity in the heart appeared to be significantly increased (Fig. 4.4B). This was accompanied by increased gene expression levels of glycolytic enzymes such as LDHA, HK1 and 2, and GLUT1 (Fig. 4.5). On protein level, this was confirmed by increases of both LDHA and HK1 following TAC (Fig. 4.7). The increased glycolytic activity after TAC is in line with several other studies linking pressure overload-induced cardiac hypertrophy to increased glycolysis ${ }^{122,136,137}$. In response to chronic volume overload, glycolytic activity in the heart displayed only a tendency to being increased compared to Sham controls, which did not reach statistical significance (Fig. 4.4B). With respect to cardiac expression of glycolytic enzymes, only HK1-mRNA levels were increased following volume overload (Fig. 4.5B). Thus, glycyolysis in the heart following chronic volume overload appeared to be only slightly enhanced, if at all. A the study by El Alaoui-Talibi et al., however, reported significant and functionally relevant increases in cardiac glycolysis following volume overload $^{120}$. There are several differences between experimental setups, which might explain the different findings with respect to levels of glycolysis. Firstly, their study was carried out in rats 
with metabolic analysis performed three months after aortocaval fistula surgery. At that time point, systolic function has presumably deteriorated already leading to different findings on glycolytic activity. Furthermore, their metabolic analysis was performed ex vivo using isolated heart perfusion experiments. By using a proteomic approach, a different study reported an increased expression of $\beta$-enolase in mice following Shunt, which could suggest higher glycolytic activity $^{138}$. Additionally, significantly decreased levels of lactate dehydrogenase (LDH) were reported, implying a shift towards more oxidative glucose metabolism in response to volume overload. However, their study focused on cardiac protein expression at an earlier time point (one week after surgery) and was carried out in $\mathrm{FVB} / \mathrm{N}$ mice rather than $\mathrm{C} 57 \mathrm{~B} 1 / 6$ mice.

Increased glycolysis in response to pressure overload in the heart has been shown to be associated with augmented glucose uptake including expression of glucose transporters ${ }^{122,139,140}$. This could not be confirmed in this study, as protein expression of GLUT1 was not different after TAC compared to Sham controls (Fig. 4.7D). A study by Zhabyeyev et al. even reported a decreased glucose uptake following pressure overload ${ }^{121}$, highlighting the inconsistent findings throughout the literature. Interestingly, GLUT1 expression was significantly increased after Shunt (Fig. 4.7D). This seems to be contradictory to the previous findings. However, whether this is really of functional relevance cannot be concluded as protein expression per se does not necessarily correlate with its function. This is why enzyme expression analysis needs to be put into context with other methodologies in order to comprehensively assess cardiac metabolism.

Data on glucose oxidation and pyruvate dehydrogenase (PDH) activity during cardiac hypertrophy and heart failure are also inconsistent, ranging from decreased or unchanged to increased levels in various rat models of cardiac hypertrophy ${ }^{141-145}$. Using the in vivo ${ }^{13} \mathrm{C}$ labelling approach through administration of $\left[\mathrm{U}_{-}{ }^{13} \mathrm{C}\right]$ glucose and subsequent flux analysis, mice subjected to chronic pressure overload reavealed significant increases in metabolic flux through $\mathrm{PDH}$ in the heart, suggesting increased PDH activity. Following chronic volume overload, PDH activity remained unchanged compared to respective controls (Fig. 4.9B) highlighting distinctive differences in PDH-dependent glucose oxidation between different animal models. Thus, the discrepancy throughout the literature migh at least partially be attributed to the usage of different animal models of cardiac hypertrophy and heart failure respectively. Gene and protein expression levels of enzymes involved in PDH activity were inconsistent following TAC and Shunt. Gene expression of PDH was increased only after chronic pressure overload without changes in pyruvate deyhrogenase kinases 1 and 4 (PDK1 and 4) expression, which negatively regulate PDH 
activity. Chronic volume overload, however, caused decreases in PDK4, but did not alter PDH and PDK1 gene expression (Fig. 4.10). Protein expression of these enzymes remained unchanged in all four experimental groups (Fig. 4.11A-D). Metabolic flux through pyruvate carboxylase (PC) and malic enzyme (ME) were also enhanced after chronic pressure suggesting increased anaplerosis (Fig. 4.9C). Studies from pressure overload-induced cardiac hypertrophy in both rats and mice also reported increases of anaplerotic flux compared to controls ${ }^{40,123}$. Again, volume overload did not change the level of anaplerosis in the heart (Fig. 4.9C). Protein levels of PC remained unchanged in both stress models (Fig. 4.11A,E). Taken together, cardiac glucose metabolism seems to be generally enhanced through chronic pressure overload, revealed by a novel in vivo ${ }^{13} \mathrm{C}$-labelling strategy. Chronic volume overload did not induce these changes to a similar extent with only glycolysis being moderately increased. This implicates an increased reliance on glucose-derived substrates for fueling cardiac mitochondria in response to pressure rather than volume overload. Discrepancies with respect to gene and protein expression levels of metabolic enzymes underline the importance of enzyme activity assessment through flux analysis.

Flux of labelled isotopomers from administered $\left[\mathrm{U}-{ }^{13} \mathrm{C}\right]$ glucose through glutamine synthetase (GS) into glutamine was also increased in the heart following chronic pressure, but not volume overload (Fig. 4.12B). This was in line with increased protein expression levels of GS following TAC (Fig. 4.14A,B). Glutamine is one of two substrates for glucosamine:fructose-6-phosphate aminotransferases 1 and 2 (GFAT 1 and 2) to produce glucosamine-6-phosphate. This represents the rate limiting step in the hexosamine biosynthetic pathway (HBP). The HBP leads to production of $\mathrm{N}$-acetylglucosamine (GlcNAc), which can be added to various proteins in an $\mathrm{O}$ linked manner (O-GlcNAcylation) (Fig. 4.13). The unique aspect of the HBP is its reliance on inputs from a variety of metabolic pathways including glucose metabolism, amino acid metabolism, fatty acid metabolism and nucleotide metabolism ${ }^{146-148}$. The focus of this study, however, was on the role of glucose-derived glutamine. Cardiac protein expression levels of GFAT2, which is the main cardiac isoform, were enhanced after chronic pressure overload suggesting HBP activation. Indeed, this was associated with significant increases in general OGlcNAcylation protein modifications. None of these changes occurred in response to chronic volume overload (Fig. 4.14A,D,E). GFAT1 is mainly expressed in the pancreas, placenta and testis $^{135}$ and therefore its protein expression levels in the heart remained unchanged following both chronic pressure and volume overload (Fig. 4.14A,C). O-GlcNAcylation has been shown to 
be increased in the heart in animal models of pressure overload as well as humans suffering from aortic stenosis $^{133,149}$. It has been implicated in the development of cardiac hypertrophy as it was shown to be required for pro-hypertrophic NFAT (nuclear factor of activated T-cells) activation in a murine pressure overload model ${ }^{134}$. Depressing of O-GlcNAcylation blunted the NFAT activation and subsequent cardiomyocyte hypertrophy. From a functional point of view, NFAT activation has been linked with pathological, but not physiological hypertrophy ${ }^{150}$, suggesting a rather detrimental role for O-GlcNAcylation in the heart during pressure overload. On the other hand, GlcNAcylation has been demonstrated to protect mice from cardiac damage in a myocardial infarction model ${ }^{151}$. In the reverse experiment, loss of GlcNAcylation exacerbated infarct-induced heart failure. Thus, the precise role of O-GlcNAcylation during cardiac stress is still unclear. Whether the detected increase after TAC contributes to the systolic impairment or results as a consequence from cardiac dysfunction cannot be elucidated. However, in the wellcompensated stage of chronic volume overload, the HBP does not seem to be relevant in the heart with respect to cardiac remodelling and function.

Glutamine can also be used as substrate for glutathione biosynthesis, if converted to glutamate, a reaction catalyzed by glutaminase (Fig. 4.13). Two weeks of pressure overload caused an increase in cardiac protein expression of glutaminase, which was not detected in response to chronic volume overload (Fig. 4.15A,B). This is also supported by the decrease in the reduced versus oxidized glutathione ratio under pressure overload conditions (Fig. 4.15C), which might indicate a greater need for glutathione to be synthesized in order to buffer increased amounts of oxidative stress. This might be a compensatory mechanism, as chronic depletion of glutathione has been shown to exacerbate pathological remodelling and cardiac dysfunction in a murine model of pressure overload ${ }^{132}$. In chronic volume overload, increased glutathione bioavailability might not be necessary due to a compensated redox environment in the heart, as indicated by an unchanged reduced versus oxidized glutathione ratio (Fig. 4.15C).

A general trend towards increased gene expression of enzymes involved in fatty acid synthesis (FAS) was found in response to pressure overload, mRNA levels of fatty acid oxidation (FAO)related enzymes remained unchanged following both pressure and volume overload (Fig. 4.17AE). The increase in cardiac gene expression levels of FAS enzymes after TAC might be a mechanism to compensate for the decreased fatty acid utilization, a consistently reported characteristic during heart failure development ${ }^{34}$. Chronic volume overload did not induce any changes in gene expression levels of enzymes involved in FAS (Fig. 4.17C-E) suggesting that 
utilization of fatty acids might still be physiological; hence, there would be no need to increase FAS. However, these conclusions are based on gene expression levels only. Therefore, it would be interesting to assess cardiac fatty acid metabolism after administration of ${ }^{13} \mathrm{C}$-labelled palmitate with subsequent flux analysis. This would give new insights into fatty acid utilization under chronic haemodynamic stress and expand the current knowledge about pressure versus volume-induced changes in cardiac metabolism.

In conclusion, this study provides novel data on the assessment of cardiac glucose metabolism using an in vivo ${ }^{13} \mathrm{C}$-labelling strategy in mice following pressure versus volume overload. Chronic pressure overload induces much more complex alterations in cardiac glucose metabolism including glycolysis, TCA cycle metabolism and glutamine synthesis compared to chronic volume overload. Whether these changes contribute to cardiac dysfunction or result as a consequence of it needs further investigation. This study also highlights that relying on gene and protein expression levels of metabolic enzymes alone is insufficient for a comprehensive assessment of cardiac metabolism. 


\section{Results III: The role of Nox4 in cardiac remodelling during chronic volume overload}

\subsection{Introduction}

Cardiac remodelling occurs in response to a variety of stressors in the heart. One of these stressors is haemodynamic load, which can be divided into pressure and volume overload. This differentiation is clinically relevant as certain cardiovascular diseases are associated with certain types of overload: pressure overload typically occurs in aortic stenosis and hypertension whereas volume overload is present in aortic and mitral regurgitation ${ }^{152-154}$. Chronic pressure and volume overload induce concentric versus eccentric remodelling respectively ${ }^{26}$. Distinct signalling pathways are likely to be involved in these responses but they are incompletely defined. Chronic pressure overload in mice has been shown to induce a more maladaptive cardiac phenotype with signs of fibrosis, apoptosis, inflammation, systolic dysfunction and higher mortality compared to chronic volume overload. The adaptive response in volume overload is associated with increased cardiac Akt activation, whereas pressure overload causes activation of calcium/calmodulindependent protein kinase II (CaMKII) ${ }^{27}$.

Oxidative stress has long been implicated in the pathophysiology of cardiovascular diseases including heart failure. Production of reactive oxygen species (ROS) correlates with the severity of heart failure in both humans and animal models ${ }^{155}$. However, clinical trials using antioxidant vitamins to treat cardiovascular diseases were unsuccessful ${ }^{156-158}$. One reason may be that ROS might exert complex functions depending on the sub-cellular location, source and the ROS species that has been generated ${ }^{65,159}$. Not all ROS effects are necessarily detrimental; in particular, specific signalling induced by ROS ("redox signalling") can be beneficial ${ }^{48,49,73}$. It is therefore not surprising that non-specific scavenging with antioxidant vitamins was not beneficial in clinical trials. In this context, NADPH oxidases (Noxs) have been gaining interest over the past two decades. They are different from other ROS-generating enzymes as ROS production by transferring electrons from NADPH to molecular oxygen is their primary function. Nox enzymes are important for redox signalling in several tissues. Of the seven known Nox family isoforms, Nox 2 and Nox 4 are the predominant ones in the heart ${ }^{160}$. Nox2, mainly located at the plasma membrane, produces superoxide and mediates angiotensin II (Ang II)-induced maladaptive cardiac remodelling ${ }^{69}$. Nox4, however, is located intracellularly, is regulated via its abundance as 
it is constitutively active, produces predominantly hydrogen peroxide ${ }^{161}$ and has been recently shown to be beneficial in the cardiovascular system under different stress conditions. In a femoral artery ligation model in mice, Nox 4 was shown to protect the vasculature from ischemic damage ${ }^{162}$. Zhang et al. demonstrated a protective role for Nox4 in the heart during chronic pressure overload through cardiac HIF-1 $\alpha$ (hypoxia-inducible factor $1 \alpha$ ) stabilization and increased angiogenesis in mice ${ }^{73}$. This was attributed specifically to cardiomyocyte-derived Nox4 as cardiomyocyte-specific Nox4 overexpressing mice were protected from pathological remodelling and cardiac dysfunction during chronic pressure overload. Additionally, under the same stress conditions, activation of the transcription factor Nuclear factor erythroid-derived 2like 2 (Nrf2), which is a key regulator of cytoprotective processes, has been shown to be Nox4dependent. In more detail, cardiac protection during chronic pressure overload in cardiomyocytespecific Nox4 overexpressing mice was markedly reduced through an additional Nrf2-deletion ${ }^{163}$. Using the aortocaval fistula (Shunt) model in mice, this study investigated a potential role for endogenous Nox4 in the cardiac response to volume overload as this is currently unknown. It was aimed to get a more detailed understanding of cardiac signalling during volume overload and eccentric remodelling respectively. 


\subsection{Assessment of Nox4-involvement in cardiac eccentric remodelling following two weeks of volume overload in mice}

As NADPH Oxidase-4 (Nox4) activity is regulated via its abundance, cardiac Nox4 expression levels in WT mice were assessed following two weeks of chronic volume overload induced via aortocaval fistula (Shunt) surgery. For a more detailed understanding, global Nox4-null mice were intensively studied and compared to WT littermates with respect to cardiac remodelling and mechanisms in response to chronic volume overload. As a reverse approach, volume overloadinduced cardiac remodelling was also assessed in cardiomyocyte-specific Nox4 overexpressing mice and compared to WT littermates.

\subsubsection{Expression profiles of NADPH oxidases and related genes in the heart following chronic volume overload}

Two weeks of volume overload in WT mice significantly induced cardiac gene expression of Nox4 (1.6 fold, p<0.05) (Fig. 5.1A) without affecting Nox2 and Nox subunits (p22 $2^{\text {phox }}, \mathrm{p} 40^{\text {phox }}$, p47 $7^{\text {phox }}$, p67 $7^{\text {phox }}$ )-mRNA levels compared to Sham controls (Fig. 5.1B-F).

A

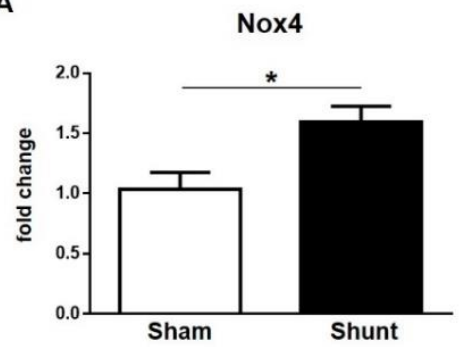

D

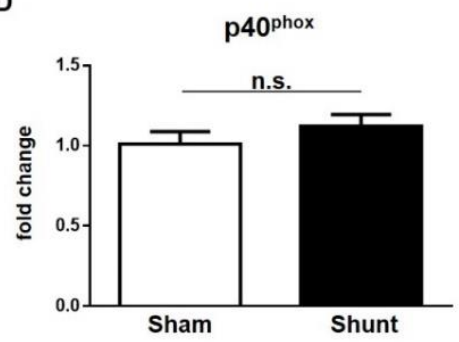

B

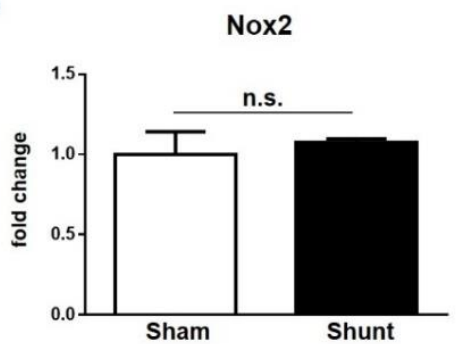

E

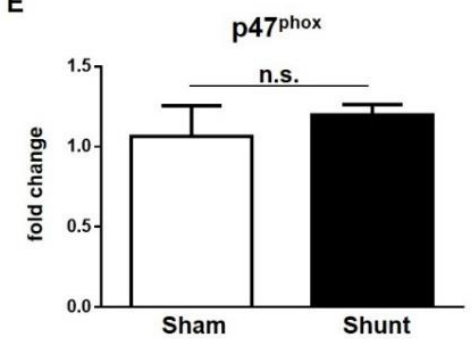

C

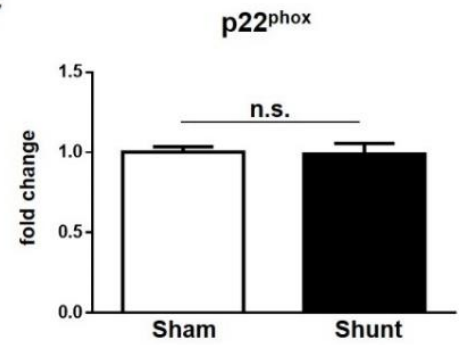

$\mathbf{F}$

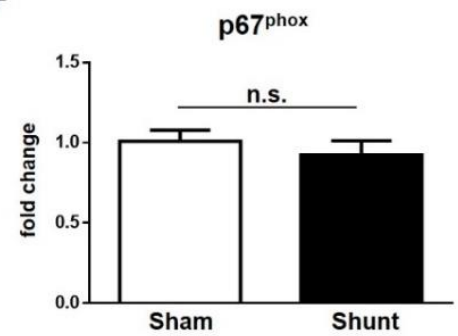

Figure 5.1: Cardiac gene expression of NADPH oxidases (Noxs) and subunits in WT mice following two weeks of volume overload. Shunt surgery was performed in age-matched, male WT mice and compared to respective Sham controls. Cardiac mRNA levels of Nox4 (A), Nox2 (B) and Nox subunits (p22 $2^{\text {phox }}, \mathrm{p} 40^{\text {phox }}, \mathrm{p} 47^{\text {phox }}$ and p67 $7^{\text {phox }}$ ) (C-F) were measured two weeks post surgery using qRT-PCR. Values are presented as fold change compared to respective Sham controls, GAPDH was used for normalization. $\mathrm{n}=5$ /group; data are presented as mean \pm SEM; * p<0.05, n.s.: not significant between Shunt and respective Sham controls using unpaired Student's t-test. 
This was confirmed on protein level as cardiac Nox4 expression was significantly increased (2.0 fold, $\mathrm{p}<0.01$ ), whereas Nox2 remained unchanged in the heart following chronic volume overload compared to respective controls (Fig. 5.2A-C). Nox2 needs to be activated through membrane translocation and binding of cytosolic subunits including $\mathrm{p} 47^{\text {phox }}$; thus, Nox2 activation in the heart following Shunt was assessed by Western Blotting for $\mathrm{p} 47^{\text {phox }}$ in the membrane-enriched fraction, where Nox 2 is located. As with total Nox 2 expression levels in whole heart lysates, Nox 2 activation was found to be also not significantly different in the heart after two weeks of volume overload compared to controls (Fig. 5.2D,E). There even appeared to be a trend towards less Nox 2 activation following chronic volume overload.

A
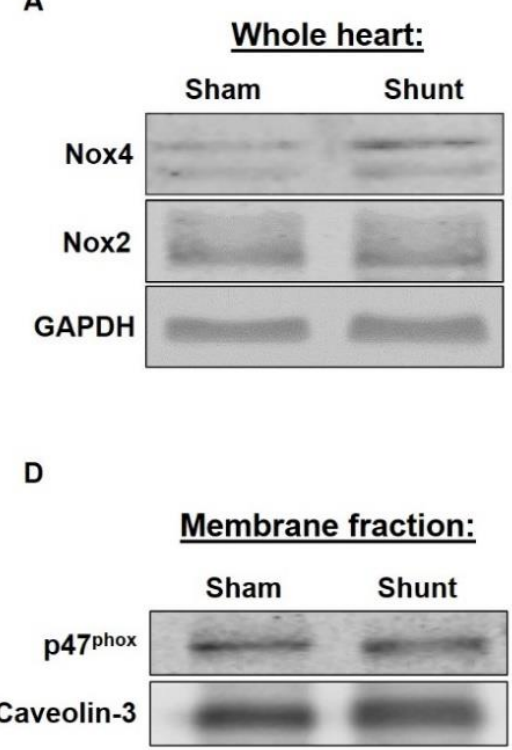

B

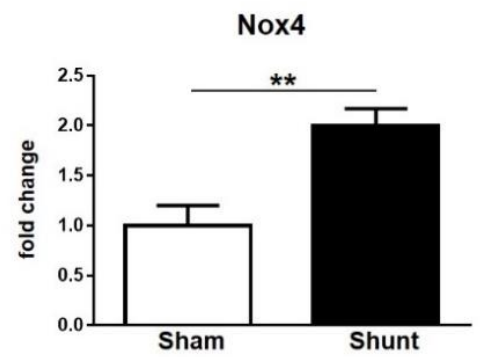

E
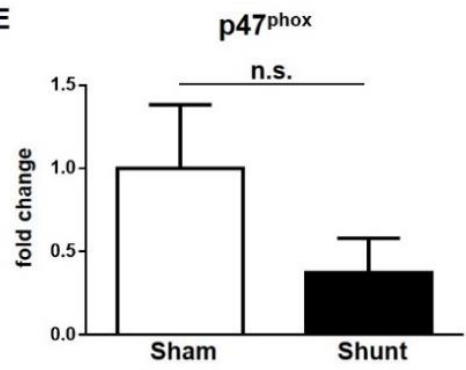

C

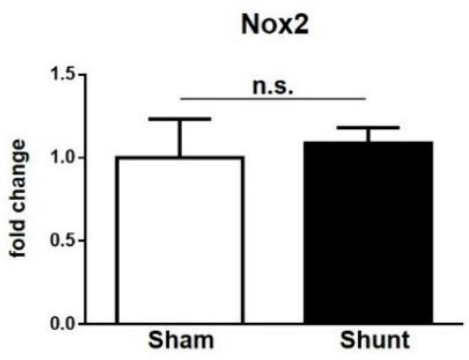

Figure 5.2: Cardiac protein expression and activation of NADPH oxidases (Noxs) in WT mice following two weeks of volume overload. Shunt surgery was performed in age-matched, male WT mice and compared to respective Sham controls. Cardiac protein levels of Nox4 and Nox2 were measured two weeks post surgery via Western Blotting using whole heart lysates. Representative images are shown in $\mathbf{A}$, densitometric quantification in $\mathbf{B}$ and C. Cardiac Nox 2 activation was assessed by detection of $\mathrm{p} 47^{\mathrm{phox}}$ in the membrane-enriched fraction. Representative image in $\mathbf{D}$, densitometric quantification in E. Values are presented as fold change compared to respective Sham controls, GAPDH was used as loading control for whole heart lysates, caveolin-3 for membrane fractions. $\mathrm{n}=4$ /group; data are presented as mean $\pm \mathrm{SEM} ; * * \mathrm{p}<0.01$, n.s.: not significant between Shunt and respective Sham controls using unpaired Student's t-test. 
Thus, chronic volume overload seems to specifically induce Nox4 expression in the heart. One downstream target of Nox4 is Nrf2 (Nuclear factor erythroid-derived 2-like 2), which has been shown to mediate cardiac protection during chronic pressure overload ${ }^{163,164}$. However, two weeks of volume overload did not change mRNA levels of three major Nrf2 targets Gclc (glutamatecysteine ligase, catalytic subunit), Gsta2 (glutathione S-transferase A2) and Txnrd1 (thioredoxin reductase 1) compared to controls (Fig. 5.3A-C). Therefore, a potential Nox4-involvement in the cardiac response to chronic volume overload is likely to be Nrf2-independent.

A

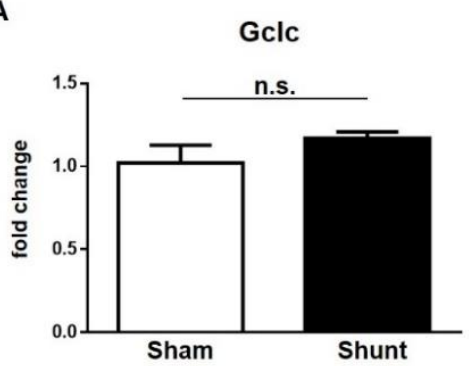

B

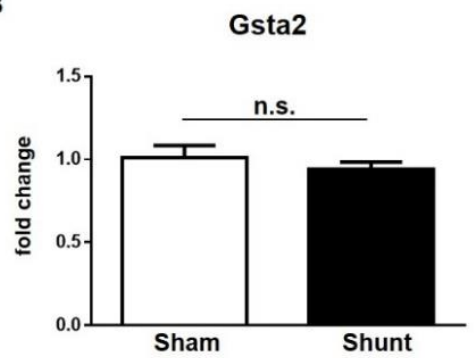

C

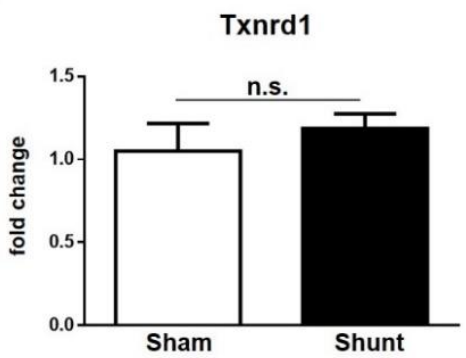

Figure 5.3: Cardiac gene expression of Nrf2 (Nuclear factor erythroid-derived 2-like 2) targets in WT mice following two weeks of volume overload. Shunt surgery was performed in age-matched, male WT mice and compared to respective Sham controls. Cardiac mRNA levels of Nrf2 targets such as Gclc (glutamate-cysteine ligase, catalytic subunit) (A), Gsta2 (glutathione S-tranferase A2) (B) and Txnrd1 (thioredoxin reductase 1) (C) were measured two weeks post surgery using qRT-PCR. Values are presented as fold change compared to respective Sham controls, GAPDH was used for normalization. $n=5$ /group; data are presented as mean \pm SEM; n.s.: not significant between Shunt and respective Sham controls using unpaired Student's t-test.

\subsubsection{Cardiac remodelling and hypertrophy in global Nox4-null mice and WT littermates}

\section{following two weeks of volume overload}

Transthoracic echocardiography two weeks after Shunt surgery revealed significant differences between global Nox4-null mice $\left(\mathrm{Nox}_{4}^{--}\right)$and WT littermates with respect to left ventricular eccentric remodelling compared to respective Sham controls (Fig. 5.4A). The increase in left ventricular end-diastolic diameter (LVEDD) as marker for dilation was increased in both genotypes, but signficantly less pronounced in $\mathrm{Nox}_{4}{ }^{-/}$-mice compared to WT littermates (4.6 vs $5.1 \mathrm{~mm}, \mathrm{p}<0.01$ ), each compared to respective Sham controls (Fig. 5.4B, Table 5.1). This difference in dilation together with unchanged septum thickness (Fig. 5.4C, Table 5.1) between the genotypes suggested an important role for Nox4 during eccentric remodelling. This conclusion was also confirmed by a more pronounced decrease in relative wall thickness in WT 
compared to Nox4-null mice (0.27 vs 0.30, p<0.05) (Fig. 5.4D, Table 5.1), which is indicative of more eccentric remodelling in WTs. Despite these differences in remodelling, global systolic function, measured by ejection fraction, was well-preserved and did not differ between the genotypes two weeks after Shunt surgery (Fig. 5.4E, Table 5.1).

The amount of left ventricular hypertrophy was also different between $\mathrm{Nox}_{4}{ }^{-/}$-mice and WT littermates following two weeks of volume overload. WGA (wheat germ agglutinin)-staining and quantification of cardiac cross sectional area revealed significantly smaller cardiomyocytes in Nox $4^{-/-}$-mice compared to WT littermates (322.8 vs $\left.378.8 \mu \mathrm{m}^{2}, \mathrm{p}<0.01\right)$ (Fig. 5.5A,B). This could also be confirmed on a macroscopic level as Nox $4^{-/-}$-mice displayed significantly less increase in left ventricular weight / tibia length ratio increase compared to WT littermates following Shunt $(+24.7$ vs $+42.6 \%, \mathrm{p}<0.05)$ (Fig. 5.5C). However, this difference in hypertrophy could only be detected in the left ventricle as right atrial, right ventricular as well as left atrial weight versus tibia length ratios increased to a similar extent in $N o x 4^{-/-}$-mice and WT littermates after two weeks of volume overload (Table 5.1). 
A
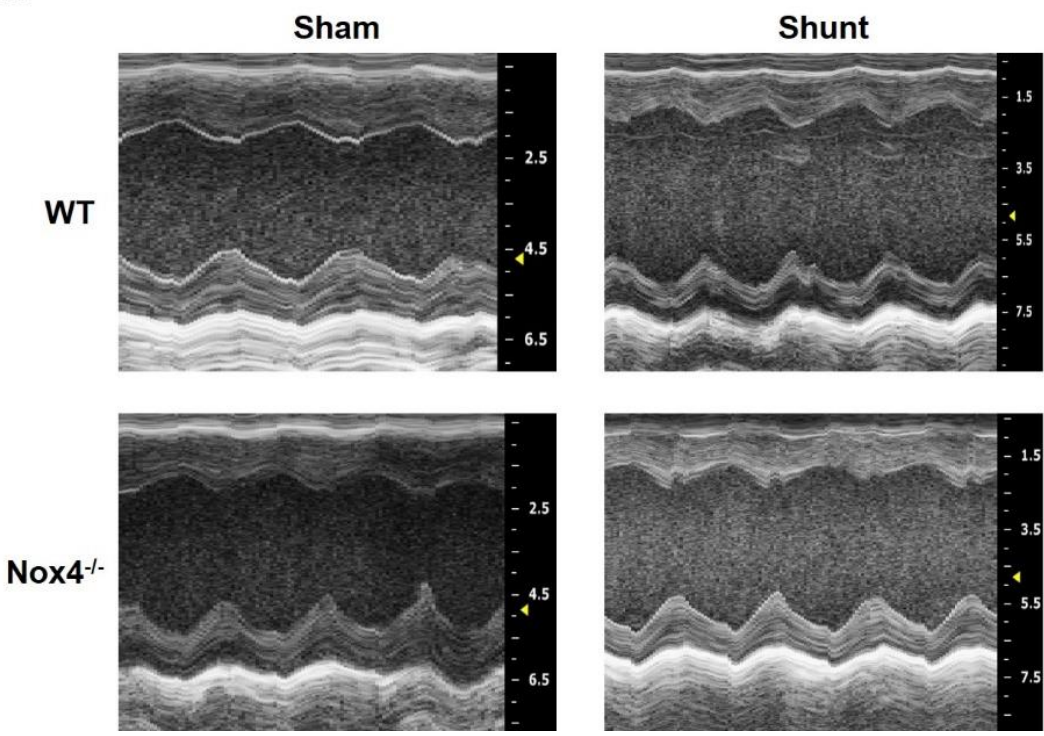

B

LVEDD

C

Septum Thickness
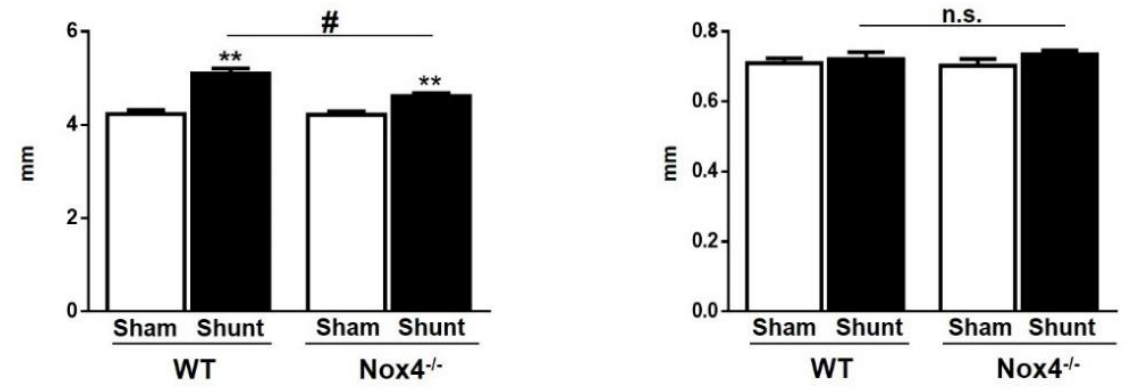

D

Relative Wall Thickness

E

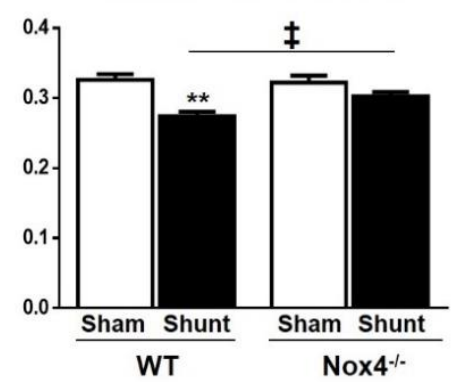

E Ejection Fraction

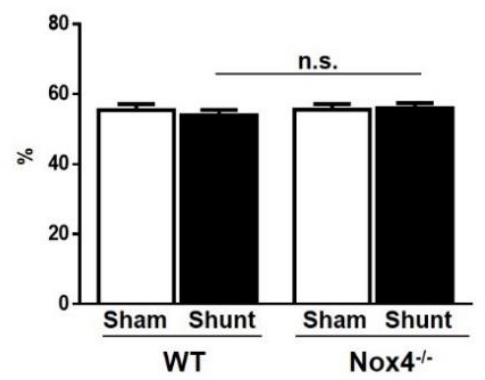

Figure 5.4: Echocardiographic assessment of left ventricular remodelling and function in $\mathrm{Nox}^{-/}{ }^{-}$-mice and WT littermates following two weeks of volume overload. Shunt surgery was performed in age-matched, male global Nox4-null mice $\left(\mathrm{Nox}^{-/-}\right)$and WT littermates compared to respective Sham controls. Representative transthoracic echocardiographic images two weeks post surgery are shown in $\mathbf{A}$, mean data for left ventricular enddiastolic diameter (LVEDD) in $\mathbf{B}$, septum thickness in $\mathbf{C}$, relative wall thickness in $\mathbf{D}$ and ejection fraction in $\mathbf{E}$. $n=9$ 12/group; data are presented as mean \pm SEM; ** $\mathrm{p}<0.01$ in Shunt versus its respective Sham control, $¥ \mathrm{p}<0.05$ significant interaction between both genotypes, \# $\mathrm{p}<0.01$ between both Shunt groups and significant interaction $(\mathrm{p}<0.01)$ between both genotypes, n.s.: not significant between both genotypes using two-way ANOVA followed by Bonferroni post-hoc test for multiple comparisons. 
A
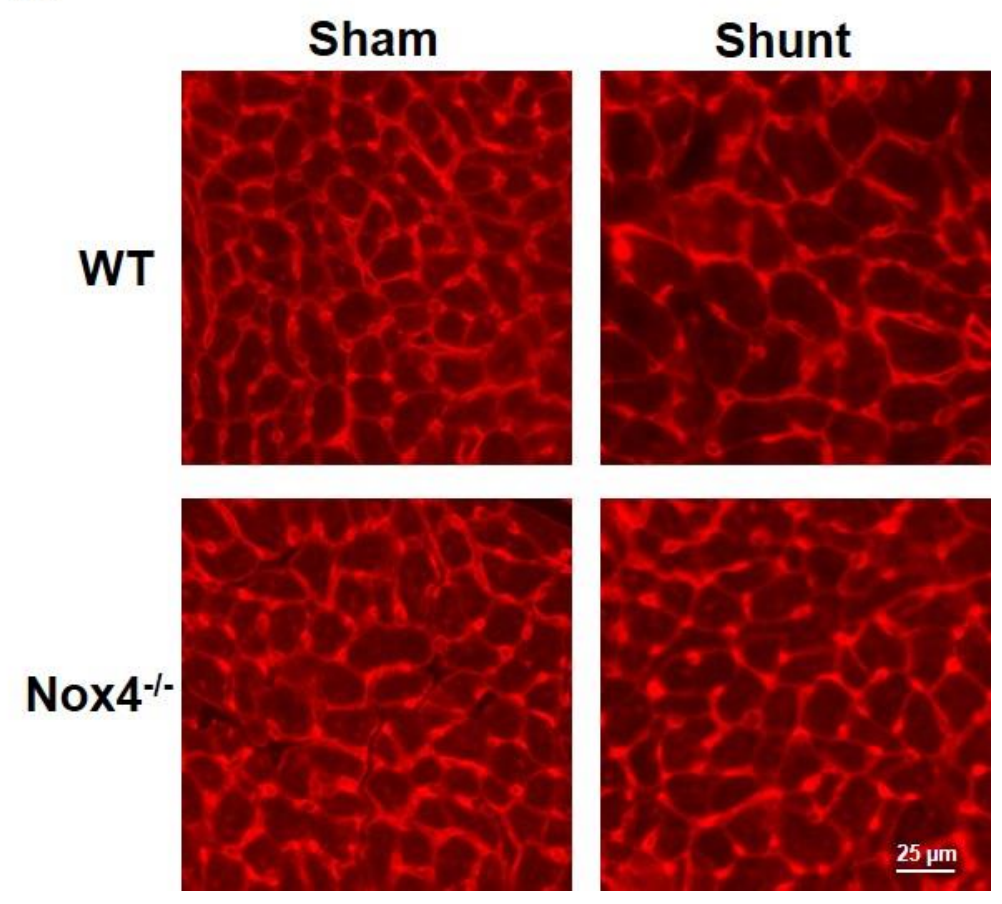

B

Cross Sectional Area

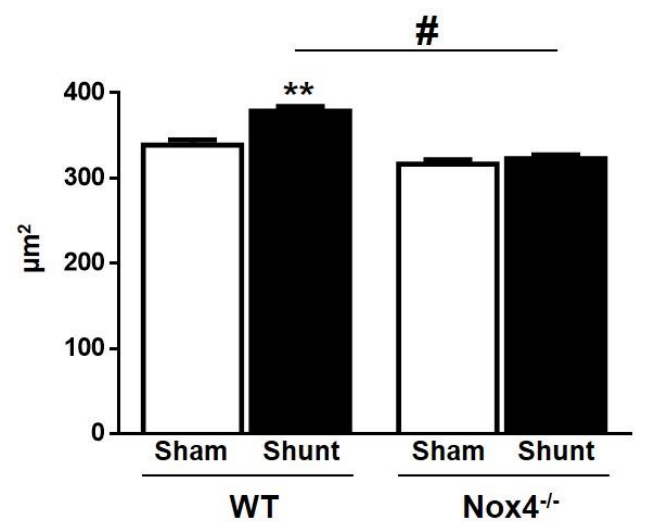

C

\section{Weight/Tibia Length}

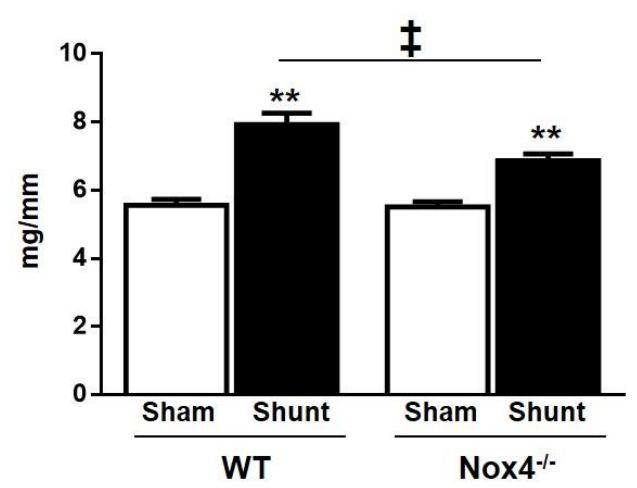

Figure 5.5: Left ventricular hypertrophy in $\mathrm{Nox}^{-/} \%$-mice and WT littermates following two weeks of volume overload. Shunt surgery was performed in age-matched, male global Nox4-null mice (Nox $4^{--}$) and WT littermates compared to respective Sham controls. Representative histological images in A are showing left ventricular myocyte cross sections stained with wheat germ agglutinin (WGA) after Shunt and respective Sham controls. Mean data for cross sectional area (CSA) are shown in $\mathbf{B}$, for left ventricular (LV) weight versus tibia length ratios two weeks after surgery in C. $n=6-12$ /group; data are presented as mean \pm SEM; ** $\mathrm{p}<0.01$ in Shunt versus its respective Sham control, $\$ \mathrm{p}<0.05$ between both Shunt groups and significant interaction $(\mathrm{p}<0.05)$ between both genotypes, \# $<<0.01$ between both Shunt groups and significant interaction $(\mathrm{p}<0.01)$ between both genotypes using two-way ANOVA followed by Bonferroni post-hoc test for multiple comparisons. 


\begin{tabular}{|c|c|c|c|c|}
\hline & \multicolumn{2}{|c|}{$\underline{\text { WT }}$} & \multicolumn{2}{|c|}{ Nox4\% } \\
\hline & Sham & Shunt & Sham & Shunt \\
\hline$n=$ & 10 & 11 & 9 & 12 \\
\hline HR [bpm] & $459 \pm 6.1$ & $471 \pm 13.0$ & $467 \pm 12.3$ & $471 \pm 12.0$ \\
\hline LVID;d [mm] & $4.2 \pm 0.09$ & $5.1 \pm 0.10^{* *}$ & $4.2 \pm 0.07$ & $4.6 \pm 0.06^{* *}$ \\
\hline LVID;s [mm] & $3.0 \pm 0.10$ & $3.7 \pm 0.09^{* *}$ & $3.0 \pm 0.08$ & $3.3 \pm 0.05$ \\
\hline LVV;d [ $\mu \mathrm{l}]$ & $77.3 \pm 2.7$ & $124.8 \pm 5.8^{* *}$ & $79.7 \pm 3.2$ & $98.6 \pm 2.9^{*}$ \\
\hline LVV;s [ $\mu l]$ & $34.8 \pm 2.6$ & $57.5 \pm 3.4^{* *}$ & $35.5 \pm 2.4$ & $43.3 \pm 1.7$ \\
\hline Septum [mm] & $0.71 \pm 0.01$ & $0.72 \pm 0.02$ & $0.70 \pm 0.02$ & $0.73 \pm 0.01$ \\
\hline Post. Wall [mm] & $0.64 \pm 0.01$ & $0.67 \pm 0.02$ & $0.65 \pm 0.02$ & $0.66 \pm 0.01$ \\
\hline LV mass [mg] & $80.9 \pm 2.8$ & $120.4 \pm 7.5^{* *}, \pm$ & $82.0 \pm 4.2$ & $99.4 \pm 2.3$ \\
\hline RWT & $0.33 \pm 0.01$ & $0.27 \pm 0.01^{* *, \mp}$ & $0.32 \pm 0.01$ & $0.30 \pm 0.01$ \\
\hline SV $[\mu l]$ & $44.3 \pm 2.0$ & $67.3 \pm 3.3^{* *}$ & $44.1 \pm 1.6$ & $55.4 \pm 2.5^{*}$ \\
\hline $\mathrm{EF}[\%]$ & $55.4 \pm 1.8$ & $54.0 \pm 1.5$ & $55.6 \pm 1.6$ & $56.0 \pm 1.4$ \\
\hline FS [\%] & $28.7 \pm 1.1$ & $28.2 \pm 1.0$ & $28.8 \pm 1.0$ & $29.3 \pm 1.0$ \\
\hline$n=$ & 11 & 11 & 10 & 12 \\
\hline Body Weight [g] & $25.9 \pm 0.52$ & $28.1 \pm 0.45$ & $26.6 \pm 0.58$ & $27.4 \pm 0.88$ \\
\hline $\mathrm{HW} / \mathrm{TL}[\mathrm{mg} / \mathrm{mm}]$ & $7.3 \pm 0.23$ & $10.5 \pm 0.44^{* *}$ & $7.3 \pm 0.17$ & $9.4 \pm 0.27^{* *}$ \\
\hline LV/TL [mg/mm] & $5.6 \pm 0.17$ & $7.9 \pm 0.33^{* *, \#}$ & $5.5 \pm 0.15$ & $6.9 \pm 0.20^{* *}$ \\
\hline $\mathrm{RV} / \mathrm{TL}[\mathrm{mg} / \mathrm{mm}]$ & $1.4 \pm 0.05$ & $1.9 \pm 0.10^{* *}$ & $1.4 \pm 0.03$ & $1.9 \pm 0.09^{* *}$ \\
\hline $\mathrm{LA} / \mathrm{TL}[\mathrm{mg} / \mathrm{mm}]$ & $0.17 \pm 0.01$ & $0.33 \pm 0.02^{* *}$ & $0.19 \pm 0.01$ & $0.32 \pm 0.01^{* *}$ \\
\hline $\mathrm{RA} / \mathrm{TL}[\mathrm{mg} / \mathrm{mm}]$ & $0.21 \pm 0.02$ & $0.32 \pm 0.02^{* *}$ & $0.19 \pm 0.01$ & $0.32 \pm 0.01^{* *}$ \\
\hline $\begin{array}{l}\text { Lung weight/TL } \\
\text { [mg/mm] }\end{array}$ & $8.7 \pm 0.12$ & $9.0 \pm 0.26$ & $8.4 \pm 0.22$ & $9.1 \pm 0.25$ \\
\hline
\end{tabular}

Table 5.1: Echocardiographic and morphometric data from Nox $4^{-/}$-mice and WT littermates following two weeks of volume overload. Shunt surgery was performed in age-matched, male global Nox4-null mice $\left(\right.$ Nox $\left.4^{-/}\right)$and WT littermates compared to respective Sham controls. Two weeks after surgery, transthoracic echocardiography was performed, followed by removal of hearts and weighing of individual cardiac chambers and lungs, each normalized to the respective tibia length (TL). HR: heart rate, bpm: beats per minute, LVID;d: left ventricular diameter in diastole, LVID;s: left ventricular diameter in systole, LVV;d: left ventricular volume in diastole, LVV;s: left ventricular volume in systole, septum: septal wall thickness, post. Wall: posterior wall thickness, RWT: relative wall thickness, SV: stroke volume, EF: ejection fraction, FS: fractional shortening, HW: total heart weight, LV: left ventricular weight, $R V$ : right ventricular weight, LA: left atrial weight, RA: right atrial weight. Data are presented as mean \pm SEM; $* \mathrm{p}<0.05, * * \mathrm{p}<0.01$ in Shunt versus its respective Sham control, $\ddagger \mathrm{p}<0.05$ between both Shunt groups and significant interaction $(\mathrm{p}<0.05)$ between both genotypes, \# $\mathrm{p}<0.01$ between both Shunt groups and significant 
interaction $(\mathrm{p}<0.01)$ between both genotypes using two-way ANOVA followed by Bonferroni post-hoc test for multiple comparisons.

\subsubsection{Cardiac stress, angiogenesis, fibrosis and apoptosis in global Nox4-null mice and WT}

\section{littermates following two weeks of volume overload}

Despite different patterns of eccentric remodelling, both Nox $4^{-/-}$-mice and WT littermates showed similar changes in cardiac mRNA levels of stress markers such as ANP (Atrial Natriuretic Peptide) (3.4 vs 3.0 fold increase, p=n.s.), BNP (Brain Natriuretic Peptide) (2.2 vs 2.4 fold increase, $p=n . s.), \quad \alpha$-skeletal actin (5.6 vs 6.7 fold increase, $p=n . s$.$) and SERCA-2 \alpha$ (Sarcoplasmic/Endoplasmic Reticulum Calcium ATPase-2 $\alpha$ ) (0.7 vs 0.7 fold change, p=n.s.) compared to respective Sham controls (Fig. 5.6A-D). Thus, these markers of the cardiac stress response appeared to be similar between the genotypes after two weeks of volume overload.

A

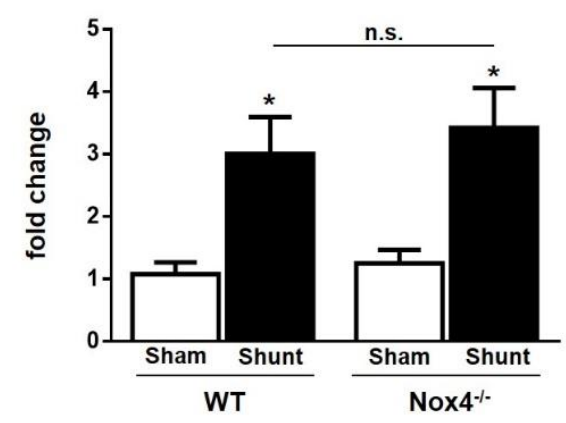

C

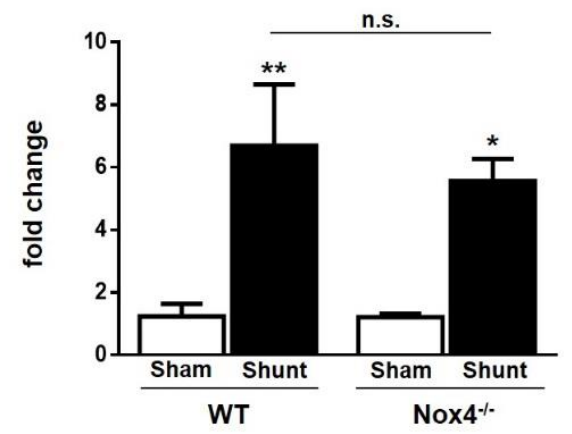

B

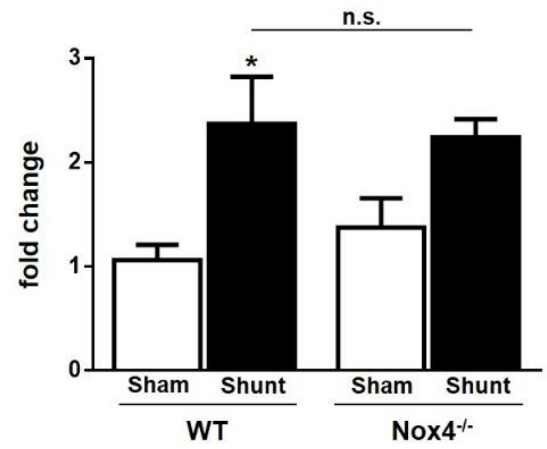

D

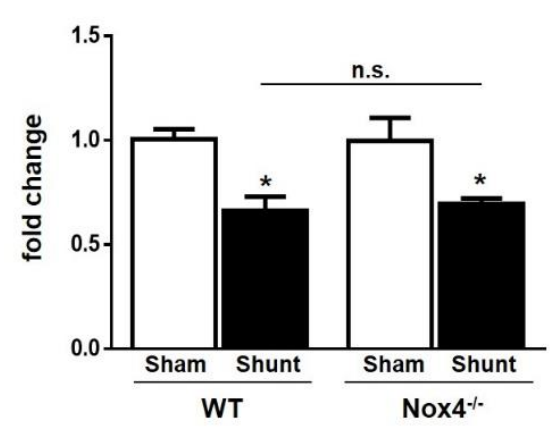

Figure 5.6: Cardiac gene expression of stress markers in Nox $4^{-/}$-mice and WT littermates following two weeks of volume overload. Shunt surgery was performed in age-matched, male global Nox4-null mice (Nox $4^{--}$) and WT littermates compared to respective Sham controls. Cardiac mRNA levels of ANP (Atrial Natriuretic Peptide) (A), BNP (Brain Natriuretic Peptide) (B), $\alpha$-skeletal actin (C) and SERCA-2 $\alpha$ (Sarcoplasmic/Endoplasmic Reticulum Calcium ATPase-2 $\alpha$ ) (D) were measured two weeks post surgery using qRT-PCR. Values are presented as fold change compared to WT Sham controls, GAPDH was used for normalization. n=6-7/group; data are presented as 
mean $\pm \mathrm{SEM} ; * \mathrm{p}<0.05, * * \mathrm{p}<0.01$ in Shunt versus its respective Sham control, n.s.: not significant between genotypes using two-way ANOVA followed by Bonferroni post-hoc test for multiple comparisons.

Isolectin B4-, Picrosirius red- and TUNEL (TdT-mediated dUTP-biotin nick end labeling)staining of cardiac cross sections revealed no changes in capillary density (Fig. 5.7), interstitial fibrosis (Fig. 5.8) and apoptosis (Fig. 5.9) through two weeks of volume overload per se and between genotypes compared to respective Sham controls.

A

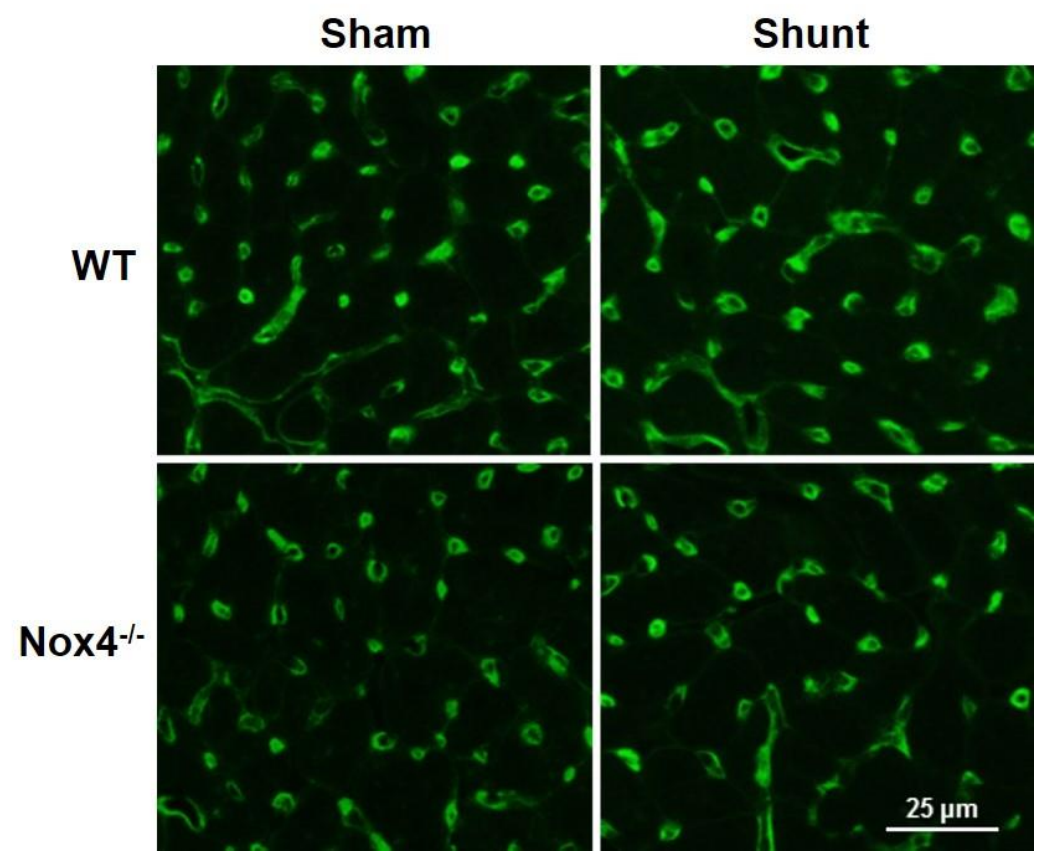

B

\section{Capillary Density}

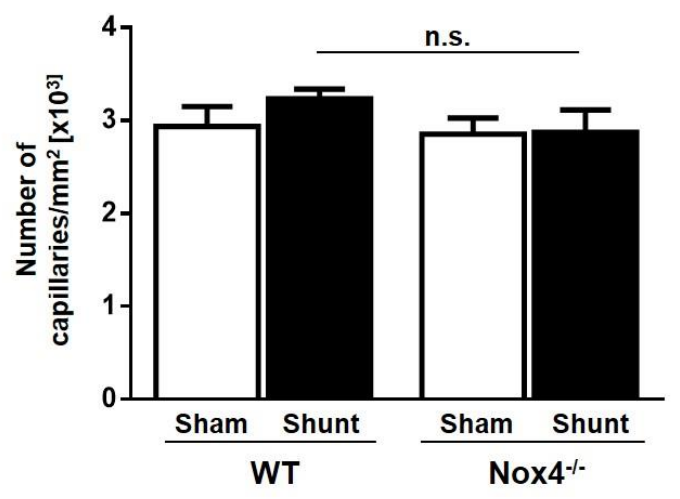

Figure 5.7: Left ventricular capillary density in Nox $4^{-/}$-mice and WT littermates following two weeks of volume overload. Shunt surgery was performed in age-matched, male global Nox4-null mice $\left(\mathrm{Nox}_{4}{ }^{-/}\right)$and WT littermates compared to respective Sham controls. Representative histological images in A illustrate left ventricular capillaries in green by isolectin B4-staining, mean data for the number of capillaries per $\mathrm{mm}^{2}$ two weeks post surgery 
are shown in B. $n=6-7 / g r o u p$; data are presented as mean \pm SEM; n.s.: not significant between both genotypes using two-way ANOVA followed by Bonferroni post-hoc test for multiple comparisons.

A

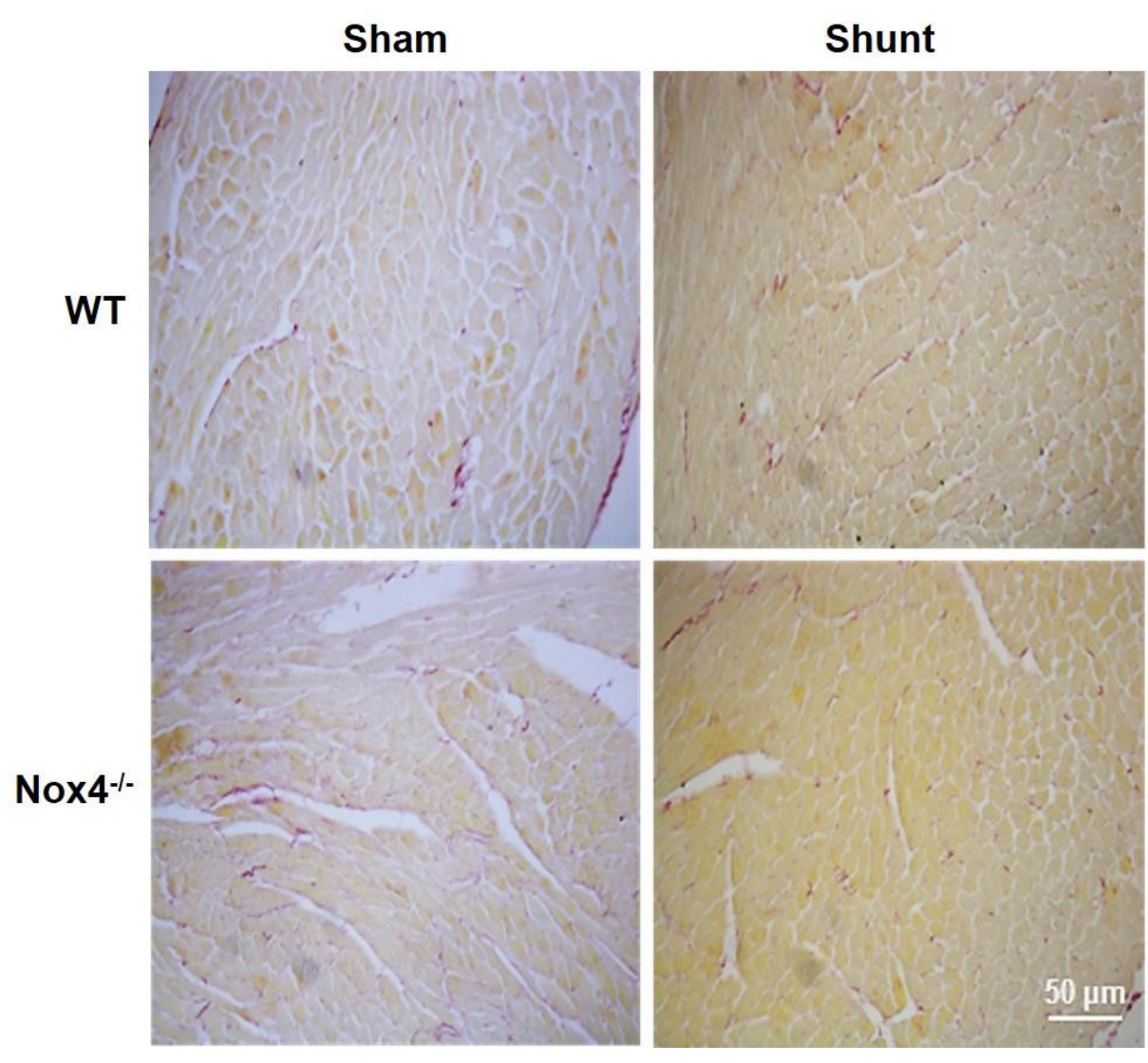

B

\section{Interstitial Fibrosis}

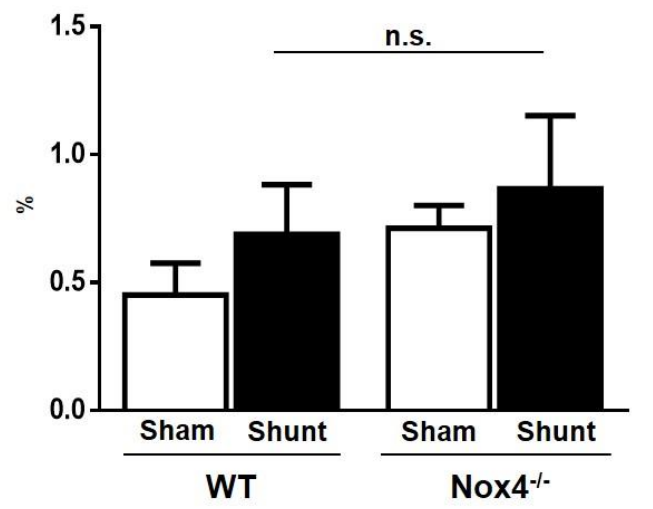

Figure 5.8: Left ventricular interstitial fibrosis in Nox4 $4^{-/-}$-mice and WT littermates following two weeks of volume overload. Shunt surgery was performed in age-matched, male global Nox4-null mice $\left(\right.$ Nox $\left.4^{-/-}\right)$and WT littermates compared to respective Sham controls. Representative histological images in A illustrate fibrotic regions (red) via Picrosirius red-staining, mean data for the fibrotic area relative to the area of the total heart slice two weeks 
post surgery are shown in B. $n=5 /$ group; data are presented as mean \pm SEM; n.s.: not significant between both genotypes using two-way ANOVA followed by Bonferroni post-hoc test for multiple comparisons.
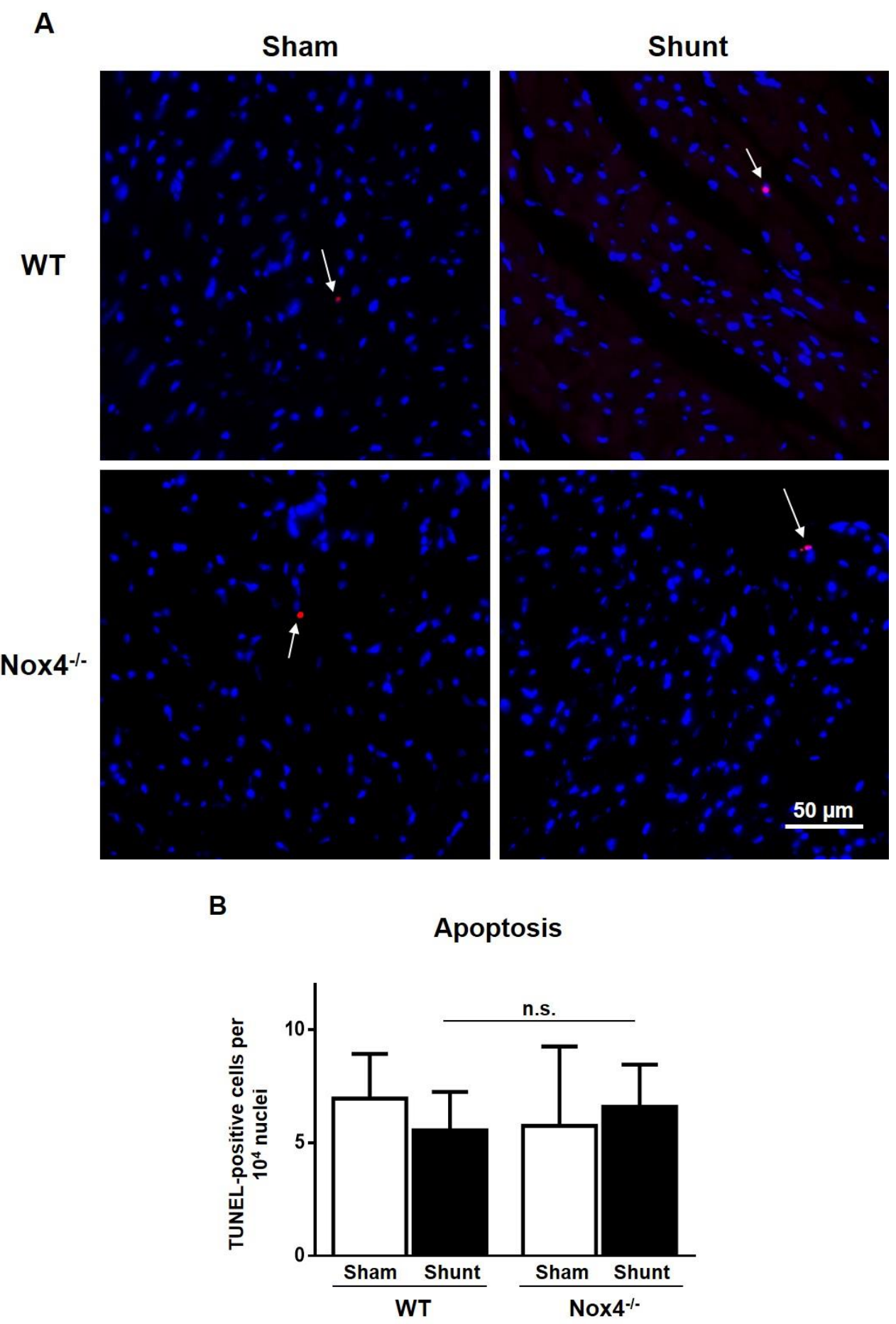

Figure 5.9: Left ventricular apoptosis in $\mathrm{Nox}^{-/-}$-mice and WT littermates following two weeks of volume overload. Shunt surgery was performed in age-matched, male global Nox4-null mice (Nox4 $4^{-/-}$) and WT littermates compared to respective Sham controls. White arrows indicate TUNEL-positive, apoptotic cells in representative histological images (A), mean data for number of apoptotic cells per counted nuclei are shown in $\mathbf{B}$. $n=4-5 /$ group; 
data are presented as mean \pm SEM; n.s.: not significant between both genotypes using two-way ANOVA followed by Bonferroni post-hoc test for multiple comparisons.

\subsubsection{Cardiac kinase and protein synthesis activation in global Nox4-null mice and WT}

\section{littermates following two weeks of volume overload}

Akt activation is known to be important in the cardiac response to volume overload ${ }^{27}$. Two weeks of volume overload induced significant differences in cardiac Akt activation between Nox4 ${ }^{-/-}$mice and WT littermates compared to respective Sham controls. Whereas WT animals showed an increase, Nox $4^{-/-}$-mice displayed a decrease in phosphorylated Akt at $\operatorname{Ser}^{473}(+29$ vs $-21 \%$, $\mathrm{p}<0.05$ ) after two weeks of volume overload (Fig. 5.10A,B). Erk1/2 is another kinase known to play a role in eccentric remodelling ${ }^{165}$. Two weeks of volume overload decreased phosphorylation levels of Erk1/2 at $\mathrm{Thr}^{202} / \mathrm{Tyr}^{204}$, but to a similar extent in Nox $4^{-/-}$-mice and WT littermates (-29 vs $-37 \%, \mathrm{p}=$ n.s.) (Fig. 5.10A,C). Taken together, this suggests a specific regulation of Akt activation by Nox4 in the heart in response to volume overload.

S6 ribosomal protein and eIF4E-BP1 (eukaryotic translation initiation factor 4E-binding protein 1) are two known protein synthesis initiators which can be regulated through Akt-dependent phosphorylation $^{166}$. In line with increased Akt activation, both these proteins showed significantly different phosphorylation levels in WT and Nox $4^{-/-}$mice. S6 ribosomal proteinphosphorylation at $\mathrm{Ser}^{235 / 236}$ was significantly increased in WTs and slightly decreased in Nox4 ${ }^{-/-}$mice (+268 vs $-19 \%$, p<0.01) (Fig. 5.11A,B), as was the hyper-phosphorylated $\gamma$-isoform of eIF4E-BP1 (+53 vs $-17 \%, \mathrm{p}<0.01)$ (Fig. 5.11A,C). These changes in phosphorylation levels suggest more cardiac protein synthesis in WT compared to Nox $4^{-/-}$-mice following chronic volume overload.

In conclusion, volume overload appears to induce eccentric hypertrophy in the heart via Akt activation and subsequent protein synthesis in a Nox4-dependent manner. 
A
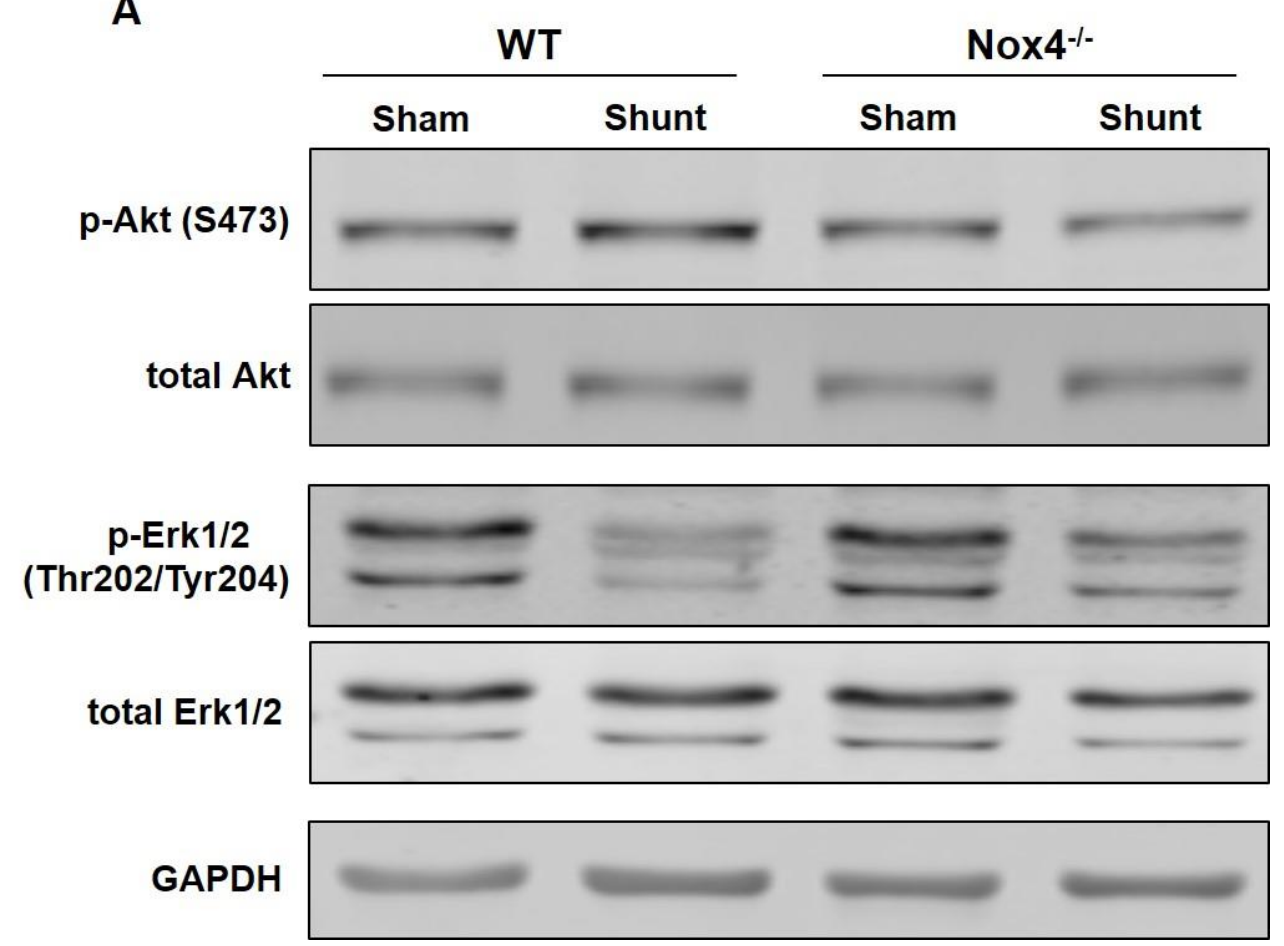

B

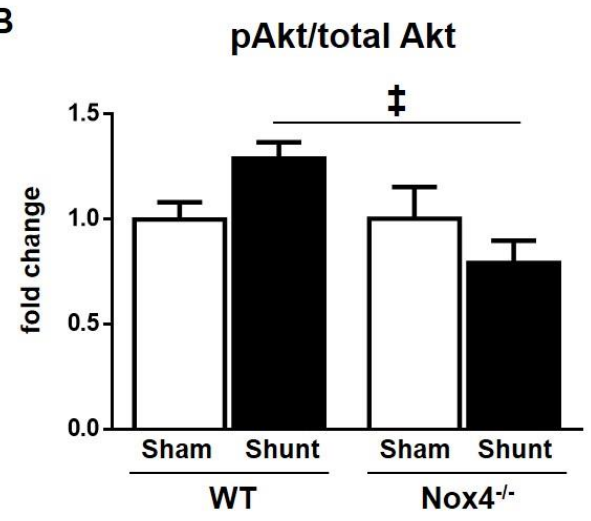

C

pErk/total Erk

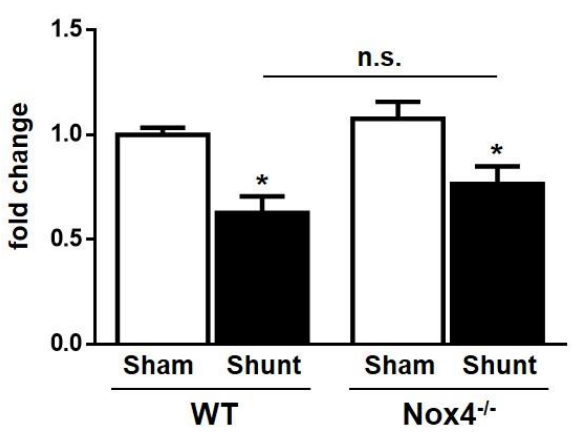

Figure 5.10: Phosphorylation of cardiac Akt and Erk1/2 in Nox $4^{-/}$-mice and WT littermates following two weeks of volume overload. Shunt surgery was performed in age-matched, male global Nox4-null mice $\left(\mathrm{Nox}^{---}\right)$and WT littermates compared to respective Sham controls. Representative Western Blot images for phospho-Akt (Ser ${ }^{473}$ ) (p-Akt), total Akt, phospho-Erk1/2 $\left(\mathrm{Thr}^{202} / \mathrm{Tyr}^{204}\right)$ (p-Erk1/2), total Erk1/2 as well GAPDH from heart lysates two weeks post surgery are illustrated in $\mathbf{A}$, densitometric quantification for phosphorylated versus total protein abundance in $\mathbf{B}$ and $\mathbf{C}$. Values are presented as fold change compared to WT Sham controls. $n=6-9 /$ group; data are presented as mean \pm SEM; $* \mathrm{p}<0.05$ in Shunt versus its respective Sham control, $\$ \mathrm{p}<0.05$ between both Shunt groups and significant interaction $(\mathrm{p}<0.05)$ between both genotypes, $n . s .:$ not significant between genotypes using two-way ANOVA followed by Bonferroni post-hoc test for multiple comparisons. 


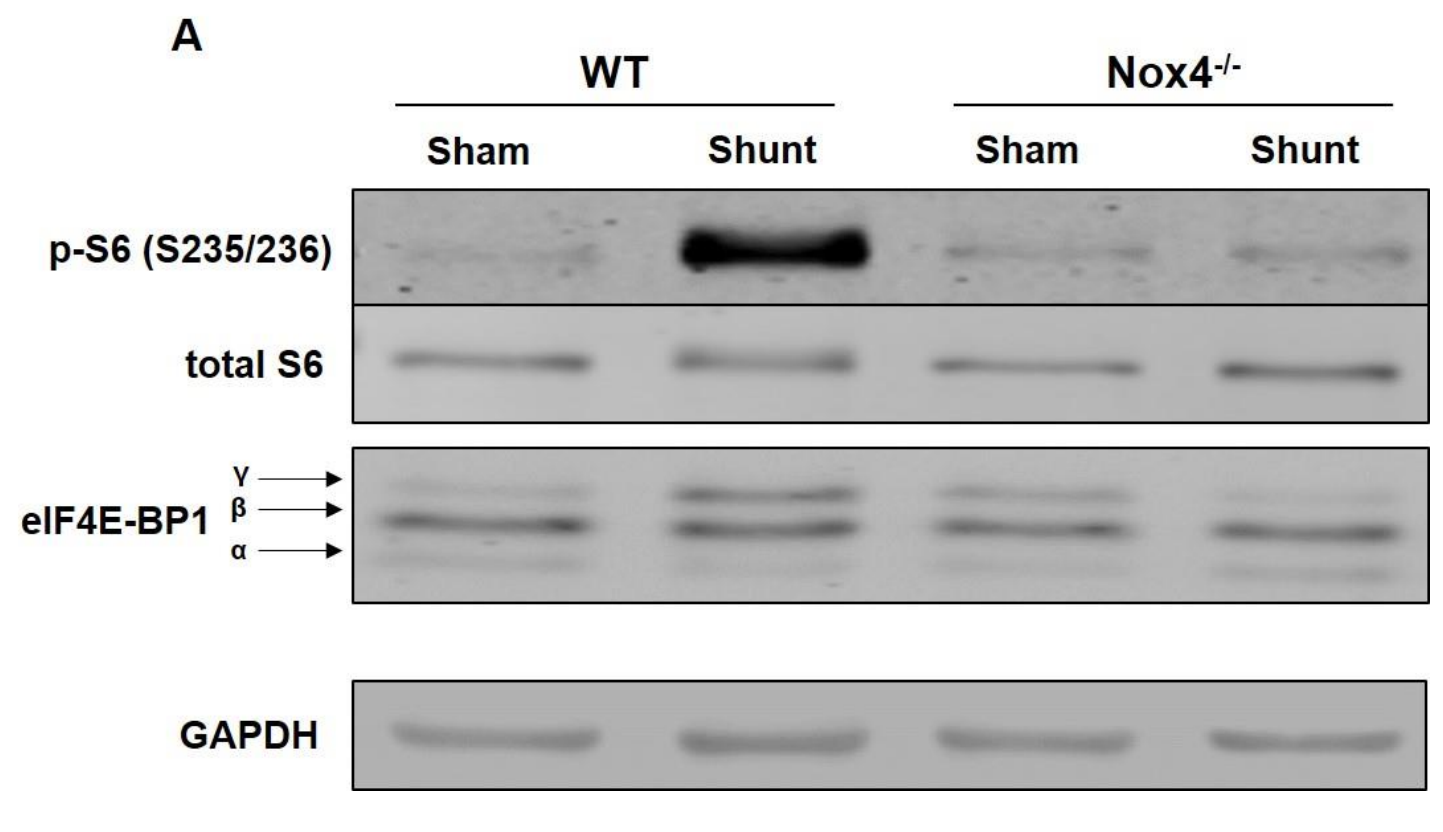

B

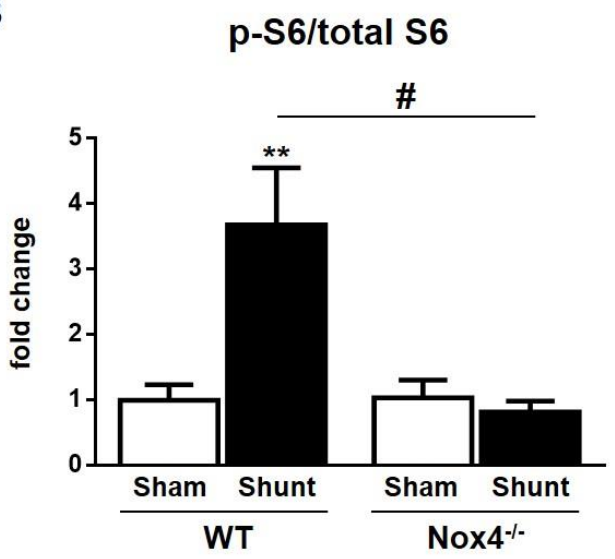

C

Y-isoform/total elF4E-BP1

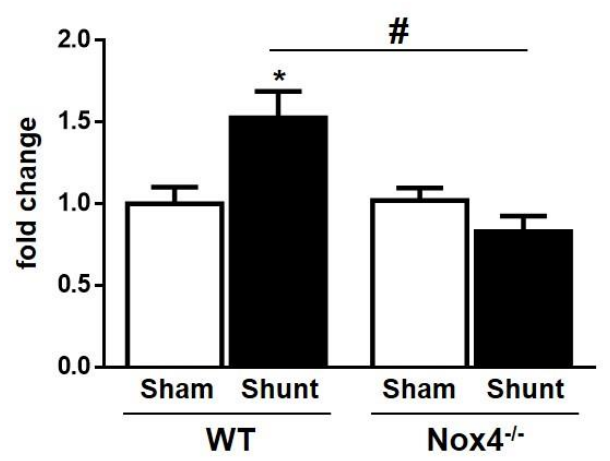

Figure 5.11: Phosphorylation of cardiac S6 ribosomal protein and eIF4E-BP1 in Nox $4^{-/-}$-mice and WT littermates following two weeks of volume overload. Shunt surgery was performed in age-matched, male global Nox4-null mice $\left(\mathrm{Nox}_{4}^{--/}\right)$and WT littermates compared to respective Sham controls. Representative Western Blot images for phospho-S6 ribosomal protein $\left(\operatorname{Ser}^{235 / 236}\right)(\mathrm{p}-\mathrm{S} 6)$, total S6 ribosomal protein, eIF4E-BP1 (divided into $\alpha, \beta$ and $\gamma$-isoform) and GAPDH from heart lysates two weeks post surgery are illustrated in A. Densitometric quantification of phosphorylated versus total $\mathbf{S 6}$ protein in $\mathbf{B}$, and the hyper-phosphorylated $\gamma$-isoform versus total eIF4E-BP1 protein in C. Values are presented as fold change compared to WT Sham controls. n=5-6/group; data are presented as mean $\pm \mathrm{SEM} ;{ }^{*} \mathrm{p}<0.05, * * \mathrm{p}<0.01$ in Shunt versus its respective Sham control, \# $\mathrm{p}<0.01$ between both Shunt groups and significant interaction $(\mathrm{p}<0.01)$ between both genotypes using two-way ANOVA followed by Bonferroni post-hoc test for multiple comparisons. 


\subsubsection{Cardiac remodelling and hypertrophy in cardiomyocyte-specific Nox4 overexpressing}

\section{mice (Nox4tg) and WT littermates following two weeks of volume overload}

Increased Nox4 expression in the heart of Nox4tg-mice was confirmed on protein level by Western Blotting (Fig. 5.12). Transthoracic echocardiography two weeks post Shunt revealed a similar increase in LVEDD (left ventricular end-diastolic dimension) in both Nox4tg-mice and WT littermates (4.9 vs $5.1 \mathrm{~mm}$, p=n.s.) compared to respective controls (Fig. 5.13A, Table 5.2). Septum thicknesses were unaltered and systolic function was preserved in all four experimental groups (Fig. 5.13B-C, Table 5.2). The amount of hypertrophy, measured as left ventricular weight versus tibia length ratio, was also similar in both Nox4tg-mice and WT littermates following two weeks of volume overload ( 7.6 vs $7.8 \mathrm{mg} / \mathrm{mm}$, p=n.s.) compared to respective Sham controls (Fig. 5.13D, Table 5.2).

Thus, forced cardiomyocyte overexpression of Nox4 does not seem to influence the cardiac response to volume overload with respect to left ventricular remodelling and hypertrophy.

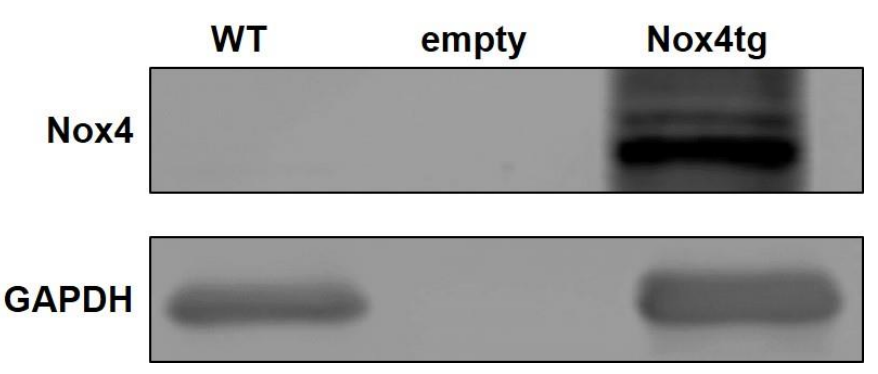

Figure 5.12: Confirmation of Nox4 protein overexpression in heart lysates from cardiomyocyte-specific Nox4 transgenic mice (Nox4tg). Nox4 was detected in heart lysates from untreated, male WT and Nox4tg-mice using Western Blot imaging. GAPDH was used as internal loading control. An empty lane without protein was included between both samples. 
A

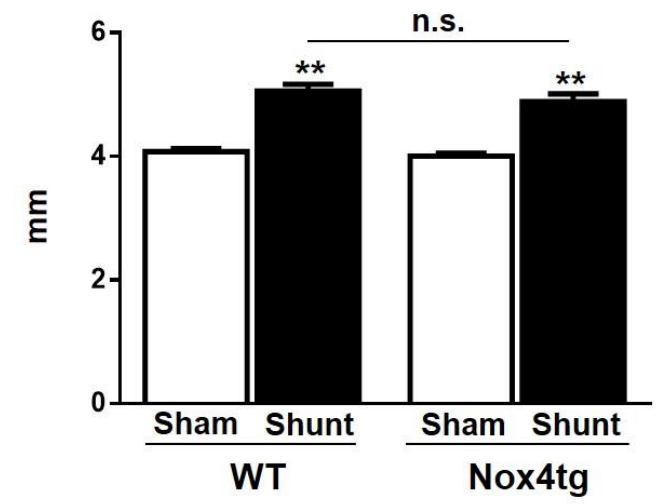

C

Ejection Fraction

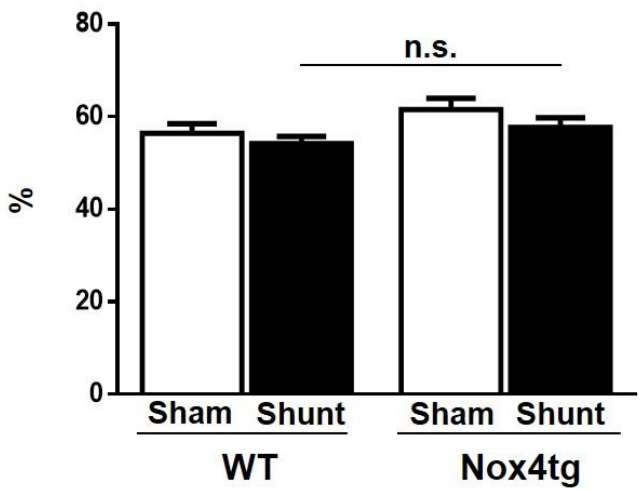

B

Septum Thickness

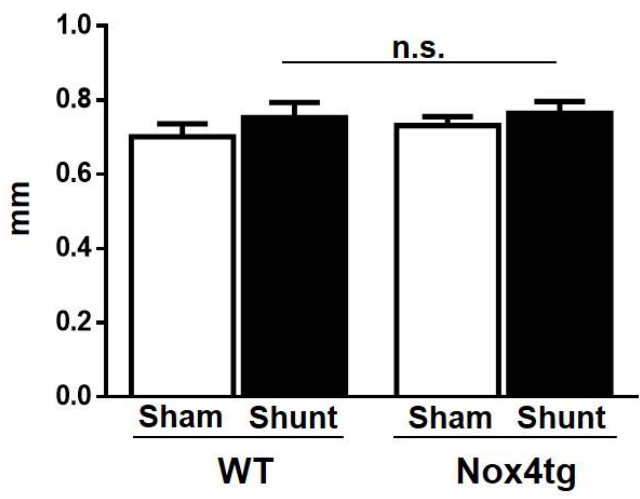

D

LV Weight/Tibia Length

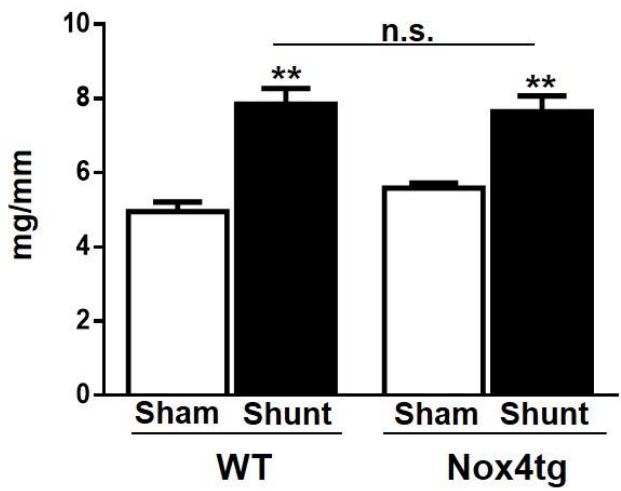

Figure 5.13: Left ventricular remodelling, function and hypertrophy in Nox4tg-mice and WT littermates following two weeks of volume overload. Shunt surgery was performed in age-matched, male cardiomyocytespecific Nox4 overexpressing mice (Nox4tg) and WT littermates compared to respective Sham controls. Transthoracic echocardiography, followed by organ harvesting was performed two weeks post surgery to assess the left ventricular end-diastolic diameter (LVEDD) (A), septum thickness (B), ejection fraction (C) and left ventricular (LV) weight versus tibia length ratio (D). $\mathrm{n}=5$-7/group; data are presented as mean $\pm \mathrm{SEM}$; ** $\mathrm{p}<0.01$ in Shunt versus its respective Sham control, n.s.: not significant between both genotypes using two-way ANOVA followed by Bonferroni post-hoc test for multiple comparisons. 


\begin{tabular}{|c|c|c|c|c|}
\hline & \multicolumn{2}{|c|}{ WT } & \multicolumn{2}{|c|}{ Nox4tg } \\
\hline & Sham & Shunt & Sham & Shunt \\
\hline$n=$ & 5 & 7 & 5 & 5 \\
\hline HR [bpm] & $456 \pm 17.7$ & $463 \pm 15.3$ & $414 \pm 2.7$ & $454 \pm 24.4$ \\
\hline LVID;d [mm] & $4.1 \pm 0.05$ & $5.1 \pm 0.11^{* *}$ & $4.0 \pm 0.04$ & $4.9 \pm 0.12^{* *}$ \\
\hline LVID;s [mm] & $2.9 \pm 0.07$ & $3.6 \pm 0.08^{* *}$ & $2.7 \pm 0.07$ & $3.4 \pm 0.15^{* *}$ \\
\hline LVV;d [ $\mu \mathrm{l}]$ & $73.5 \pm 2.1$ & $122.0 \pm 6.3^{* *}$ & $70.5 \pm 1.8$ & $112.5 \pm 6.7^{* *}$ \\
\hline LVV;s [ $\mu l]$ & $32.1 \pm 1.8$ & $55.7 \pm 3.0^{* *}$ & $27.11 \pm 1.6$ & $48.1 \pm 5.0^{* *}$ \\
\hline Septum [mm] & $0.70 \pm 0.03$ & $0.75 \pm 0.04$ & $0.73 \pm 0.02$ & $0.76 \pm 0.03$ \\
\hline Post. Wall [mm] & $0.61 \pm 0.03$ & $0.71 \pm 0.02$ & $0.65 \pm 0.03$ & $0.69 \pm 0.04$ \\
\hline LV mass [mg] & $78.4 \pm 7.5$ & $126.6 \pm 6.6^{* *}$ & $75.1 \pm 2.3$ & $120.3 \pm 8.1^{* *}$ \\
\hline RWT & $0.32 \pm 0.01$ & $0.29 \pm 0.01$ & $0.35 \pm 0.02$ & $0.30 \pm 0.02$ \\
\hline SV $[\mu \mathrm{I}]$ & $41.4 \pm 1.9$ & $66.3 \pm 4.4^{* *}$ & $43.4 \pm 2.3$ & $64.4 \pm 2.2^{* *}$ \\
\hline EF [\%] & $56.4 \pm 2.1$ & $54.1 \pm 1.6$ & $61.5 \pm 2.4$ & $57.7 \pm 2.1$ \\
\hline FS [\%] & $29.2 \pm 1.4$ & $28.2 \pm 1.1$ & $32.8 \pm 1.8$ & $30.5 \pm 1.4$ \\
\hline$n=$ & 5 & 7 & 5 & 6 \\
\hline Body Weight [g] & $24.2 \pm 1.4$ & $25.4 \pm 1.0$ & $24.6 \pm 1.2$ & $25.2 \pm 0.77$ \\
\hline HW/TL [mg/mm] & $6.7 \pm 0.35$ & $11.30 \pm 0.66^{* *}$ & $7.5 \pm 0.22$ & $10.9 \pm 0.53^{* *}$ \\
\hline $\mathrm{LV} / \mathrm{TL}[\mathrm{mg} / \mathrm{mm}]$ & $5.0 \pm 0.26$ & $7.8 \pm 0.42^{* *}$ & $5.6 \pm 0.13$ & $7.6 \pm 0.43^{* *}$ \\
\hline $\mathrm{RV} / \mathrm{TL}[\mathrm{mg} / \mathrm{mm}]$ & $1.4 \pm 0.08$ & $2.5 \pm 0.24^{* *}$ & $1.5 \pm 0.09$ & $2.4 \pm 0.18^{*}$ \\
\hline $\mathrm{LA} / \mathrm{TL}[\mathrm{mg} / \mathrm{mm}]$ & $0.17 \pm 0.02$ & $0.48 \pm 0.04^{* *}$ & $0.22 \pm 0.01$ & $0.45 \pm 0.02^{* *}$ \\
\hline $\mathrm{RA} / \mathrm{TL}[\mathrm{mg} / \mathrm{mm}]$ & $0.20 \pm 0.03$ & $0.44 \pm 0.04^{* *}$ & $0.20 \pm 0.03$ & $0.42 \pm 0.04^{* *}$ \\
\hline $\begin{array}{l}\text { Lung weight/TL } \\
\text { [mg/mm] }\end{array}$ & $8.2 \pm 0.26$ & $11.0 \pm 1.0$ & $8.3 \pm 0.27$ & $10.0 \pm 0.46$ \\
\hline
\end{tabular}

Table 5.2: Echocardiographic and morphometric data from Nox4tg-mice and WT littermates following two weeks of volume overload. Shunt surgery was performed in age-matched, male cardiomyocyte-specific Nox4 overexpressing mice (Nox4tg) and WT littermates compared to respective Sham controls. Two weeks after surgery, transthoracic echocardiography was performed, followed by removal of hearts and weighing of individual cardiac chambers and lungs, each normalized to the respective tibia length (TL). HR: heart rate, bpm: beats per minute, LVID; d: left ventricular diameter in diastole, LVID;s: left ventricular diameter in systole, LVV; d: left ventricular volume in diastole, LVV;s: left ventricular volume in systole, septum: septal wall thickness, post. Wall: posterior wall thickness, RWT: relative wall thickness, SV: stroke volume, EF: ejection fraction, FS: fractional shortening, HW: total heart weight, LV: left ventricular weight, RV: right ventricular weight, LA: left atrial weight, RA: right atrial weight. Data are presented as mean \pm SEM; $* \mathrm{p}<0.05, * * \mathrm{p}<0.01$ in Shunt versus its respective Sham control, none of parameters were significantly different between genotypes using two-way ANOVA followed by Bonferroni post-hoc test for multiple comparisons. 


\subsection{Discussion}

Nox4 expression has been shown to be induced by various stresses such as chronic pressure overload or myocardial infarction in vivo as well as hypoxia in vitro ${ }^{73}$. This study demonstrates for the first time an induction of Nox4 in the heart after chronic volume overload in vivo. Furthermore, Nox4 is shown to promote adaptive, eccentric remodelling in the heart during volume overload, at least partially via Akt-mediated activation of protein synthesis initiators. This finding is in line with previous studies from our group emphasizing a beneficial and protective role for Nox4 in the heart during chronic pressure overload ${ }^{73}, 163$. However, other studies have reported rather detrimental effects of Nox4 in the heart during cardiac stress ${ }^{167,}{ }^{168}$. In the current controversial debate about the precise role of Nox4 in the heart ${ }^{169}$, this study once more highlights the physiological importance for endogenous Nox4 during haemodynamic stress. Chronic volume overload in vivo stretches cardiomyocytes resulting in increased length ${ }^{27}$. According to our findings, this affects Nox4 without changing Nox2 expression or activation levels (Fig. 5.1, 5.2). This seems to be in contrast with a study from Prosser et al., which demonstrated increases in Nox2 activation and ROS production after cardiomyocyte $\operatorname{stretch}^{170}$. However, their experiments were performed under short-term acute physiological stretch in vitro, whereas the aortocaval fistula (Shunt) model induces pathological chronic stress in vivo. Prosser et al. investigated mechanisms in single cardiomyocytes, experiments in our study were performed in whole heart lysates and membrane-enriched fractions from heart lysates respectively. These differences might explain the different observations with respect to Nox 2 .

The maladaptive cardiac response to pressure overload compared to a more beneficial and physiological cardiac phenotype following chronic volume overload has been addressed before ${ }^{27}$. In a previous Chapter of this thesis (4.3.3), pressure overload was shown to induce a shift towards an oxidized environment in the heart as the reduced versus oxidized glutathione ratio was significantly decreased compared to controls. The same duration of volume overload, however, did not alter the cardiac redox status (Fig. 4.15C). Thus, it was tested whether the increase in Nox4 expression following volume overload might maintain the redox status in the heart through an Nrf2-mediated antioxidant response, as this mechanism is known to be protective during chronic pressure overload ${ }^{163}$. However, mRNA levels of three commonly reported Nrf2 targets Gclc (glutamate-cysteine ligase, catalytic subunit), Gsta2 (glutathione Stranferase A2) and Txnrd1 (thioredoxin reductase 1) remained unchanged after two weeks of volume overload (Fig. 5.3). Hence, Nox4-dependent Nrf2 activation does not seem to be a 
relevant mechanism in the cardiac response to volume overload. Whether the difference in the general cardiac redox status is cause or consequence for the two different phenotypes following pressure and volume overload needs further investigation. It emphasizes that ROS production, balance and source can be different depending on the cardiac stress or cardiovascular disease respectively. From this point of view, it seems logical that general antioxidant treatment in cardiovascular diseases was shown to be unsuccessful ${ }^{157}$.

Increased angiogenesis through HIF-1 $\alpha$ (hypoxia-inducible factor $1 \alpha$ ) stabilization and subsequent release of VEGF (vascular endothelial growth factor) is one of the mechanisms by which Nox 4 protects the heart from maladaptive concentric remodelling during chronic pressure overload $^{73}$. However, here it was demonstrated that this mechanism does not seem to be relevant after two weeks of volume overload. Neither Nox4-deletion nor two weeks of volume overload per se changed the capillary density in the heart (Fig. 5.7). Likewise, there were no changes in interstitial fibrosis (Fig. 5.8) or cardiac apoptosis (Fig. 5.9), again highlighting a more physiological response in the heart following two weeks of volume overload.

Akt activation is known to play an important role in the cardiac adaptation to volume overload. After 20 weeks of volume overload, Akt-deficient mice showed decreased left ventricular eccentric remodelling and hypertrophy, which was associated with impaired systolic function and higher mortality compared to WT littermates ${ }^{171}$. Our findings identified Nox4 as an upstream activator of Akt during chronic volume overload as Nox4-null mice displayed less Akt activation compared to WT littermates after two weeks of Shunt (Fig. 5.10A,B). Similar to Akt-deficient mice, this was associated with less left ventricular eccentric remodelling and hypertrophy in Nox4-null mice (Fig. 5.4, 5.5, Table 5.1). An interaction between Nox4 and Akt has been recently reported in an angiotensin II (Ang II) model, where cardiac-specific overexpression of human Nox4 in mice led to maladaptive cardiac remodelling including fibrosis through Akt activation $^{168}$. With respect to the Ang II and the Shunt model being two very different stress models, this is in contrast to our findings suggesting an adaptive response through that same mechanism. However, the study by Zhao et al. used a transgenic mouse with a 10 fold increase in cardiac protein levels of human Nox4 and 8 fold in subsequent ROS production. Such a supernormal increase in ROS might regulate signalling pathways in an artificial and nonphysiological manner. Thus, we focused on physiological mechanisms regulated by endogenous Nox4 using global Nox4-null mice. Even though prolonged Akt activation can cause cardiac dilation and systolic dysfunction ${ }^{172}$, many studies report rather beneficial roles for Akt in the 


\section{Results III}

heart. In line with our findings, Akt-deficient mice show less physiological growth in response to exercise, but an increase in maladaptive cardiac remodelling and contractile dysfunction to pathological stimuli such as chronic pressure overload or endothelin- ${ }^{173,174}$. This supports the hypothesis that Nox4-Akt-dependent eccentric remodelling is an adaptive response of the heart to volume overload.

Decreased Erk1/2-phosphorylation has been shown to be associated with eccentric remodelling in vitro $^{165}$. We could confirm these results by showing significantly less Erk1/2-phosphorylation after two weeks of volume overload (Fig. 5.10A,C). However, this does not seem to be regulated in a Nox4-dependent manner as it occurs in Nox4-null mice and WT littermates to a similar extent. Whether decreased Erk1/2-phosphorylation contributes to eccentric remodelling in a physiological or pathological manner needs further investigation.

In line with the Nox4-dependent regulation of Akt activation during volume overload, phosphorylation of S6 ribosomal protein and eIF4E-BP1 also appeared to be regulated by Nox4 (Fig. 5.11). S6 ribosomal protein and eIF4E-BP1 are two known targets of the Akt-mTOR-axis and initiate general protein synthesis upon phosphorylation. This mechanism has recently been reported to be associated with cardiac adaptation to volume overload in mice: treatment of WT mice with the mTOR-inhibitor temsirolimus reduced S6 ribosomal protein and eIF4E-BP1phosphorylation, which caused cardiac atrophy, less eccentric remodelling and higher mortality compared to vehicle treatment during chronic volume overload ${ }^{175}$. We could confirm that phosphorylation of S6 ribosomal protein and eIF4E-BP1 are increased through volume overload in WT mice, which is blunted in global Nox4-null mice.

Despite the different phenotypes in global Nox4-null mice and WT littermates, cardiomyocytespecific overexpression of Nox4 had no effect on left ventricular remodelling and hypertrophy following two weeks of volume overload compared to WTs (Fig. 5.13). This could be interpreted in two ways: 1. Cardiomyocyte-derived Nox4 is not crucial for promoting eccentric hypertrophy. The different response in global Nox4-null mice might therefore be mediated by other cell types such as fibroblasts, which represent more than $50 \%$ of all cardiac cells ${ }^{176}$. However, the majority of cardiac mass and volume is made up of cardiomyocytes. As the differences in Akt-protein synthesis-signalling were detected in this study using whole heart lysates (Fig. 5.10, 5.11), it is more likely to be a cardiomyocyte-dependent phenotype. Additionally, left ventricular hypertrophy following chronic volume overload was associated with increases in cardiomyocyte area (Fig. 5.5) without any signs of fibrosis (Fig. 5.8), again emphasizing the importance of 
cardiomyocytes in this setting. Previous studies from our group also reported cardiac protection by Nox4 to be cardiomyocyte-specific during pressure overload ${ }^{73,163} .2$. In order to promote adaptive eccentric hypertrophy following chronic volume overload, physiological increases in cardiac Nox4 levels are enough, further augmentation is redundant and might even regulate signalling pathways in an unphysiological manner, as mentioned earlier. This seems more likely to be the explanation for why Nox4tg-mice do not differ from WT littermates with respect to left ventricular remodelling following chronic volume overload.

Taken together, Nox 4 is known to reduce maladaptive, concentric remodelling during chronic pressure overload through different mechanisms. During volume overload, Nox4 seems to activate the Akt-protein synthesis-axis to promote adaptive, eccentric remodelling. This is schematically illustrated in Fig. 5.14. Nox-inhibition has long been considered as a therapeutic option in various diseases. GKT137831, a Nox 1 and Nox4 inhibitor, is currently undergoing class II clinical trials as potential treatment for diabetic nephropathy. However, most compounds lack isoform-specificity and too little is known about their exact mechanisms ${ }^{177,}{ }^{178}$. Our study confirms the importance of isoform-specificity, as inhibiting Nox4 might also attenuate adaptive processes in the heart during haemodynamic stress. This has to be considered in the development of new compounds targeting NADPH oxidases.

\section{Limitation:}

We could not detect any functional differences after two weeks of volume overload. Impairment in systolic function is known to occur very late in this model, starting after around 15 weeks ${ }^{171}$. In our hands, time points as late as 15 weeks were inappropriate due to a potential closure of the Shunt. It has been reported that a three-week maturation phase of the fistula is followed by a three-week potential failure phase ${ }^{179}$, which we have also observed in this model. 


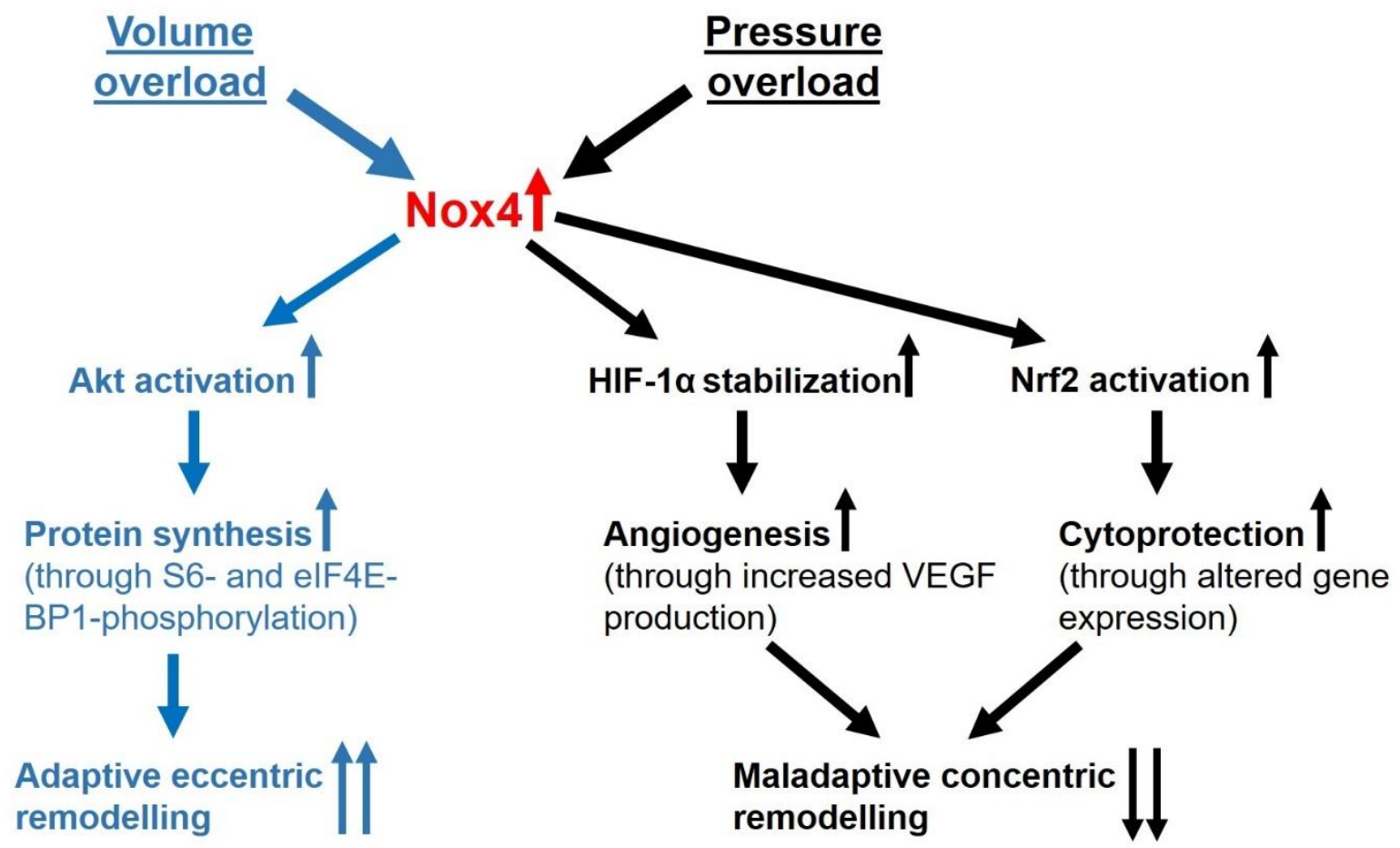

Figure 5.14: Scheme of Nox4-involvement in the regulation of cardiac remodelling during pressure and volume overload. Pressure overload and volume overload activate Nox4. In pressure overload, Nox4 increases angiogenesis through stabilization of the hypoxia-inducible factor $1 \alpha$ (HIF-1 $\alpha)$ and subsequent increases in levels of vascular endothelial growth factor (VEGF). Additionally, Nox4 increases levels of the transcription factor Nuclear factor erythroid-derived 2-like 2 (Nrf2), which induces a cytoprotective gene program. Both these mechanisms have been described to reduce detrimental, concentric remodelling ${ }^{73,163}$.

In volume overload, we could demonstrate that Nox4 promotes eccentric cardiac adaptation through Akt activation and presumably increased protein synthesis. 


\section{Concluding Discussion}

\section{Concluding Discussion}

This study provides novel data on cardiac physiology and metabolism during chronic haemodynamic stress, i.e. volume versus pressure overload. The aortocaval fistula (Shunt) is a well-established, but not frequently used model to induce volume overload in mice. As expected, Shunt as a volume overload model induced eccentric cardiac hypertrophy with left ventricular dilation. Pressure overload models such as abdominal aortic banding (AAB) or transverse aortic constriction (TAC) caused concentric hypertrophy, characterized by wall thickening (Figure 4.1). Despite these different remodelling patterns, two weeks of Shunt and TAC have been shown to induce almost identical levels of left ventricular hypertrophy (Fig. 4.2). However, functional and metabolic properties were remarkably different between both models. This was assessed by stateof-the-art echocardiography (Chapter 3 ) and a novel in vivo ${ }^{13} \mathrm{C}$-labelling strategy with [U- $\left.{ }^{13} \mathrm{C}\right]$ glucose administration followed by flux analysis using NMR-spectroscopy (Chapter 4). This in vivo approach was meant to give new insights into glucose metabolism during cardiac hypertrophy and heart failure, as this is currently under controversial debate ${ }^{34}$.

In Chapter 3, differences between volume and pressure overload-induced changes in systolic and diastolic parameters were reported and discussed. Volume overload was not associated with systolic impairment, but significantly altered diastolic parameters. These included a decreased IVRT (isovolumic relaxation time) and increases in both left atrial area and diastolic strain as signs of increased left ventricular filling pressure. These changes are usually associated with a restrictive pattern of diastolic heart failure, which is characterized by left ventricular filling primarily during early diastole due to significantly elevated left atrial pressure levels. This restrictive filling pattern is typically observed in the end-stage of progressive diastolic dysfunction and is associated with a poor prognosis in patients ${ }^{180}$. It is related to progressive left ventricular dilation and is a predictor for cardiac death in patients with prior myocardial infarction. The murine Shunt model, however, stays well-preserved for a long period of time without development of heart failure and increased mortality. This suggests the detected changes in diastolic parameters to be model-dependent through increased preload rather than reflecting functionally relevant diastolic dysfunction. This is also supported by the fact that $\mathrm{E} / \mathrm{E}^{\prime}$, the most sensitive parameter to assess diastolic impairment in humans, is not different amongst mice subjected to Shunt and Sham respectively. Additionally, we did not detect any changes in cardiac fibrosis following Shunt (Fig. 5.8). This is in line with rather physiological diastolic properties, as fibrosis is known to be a key contributor to diastolic dysfunction ${ }^{181,}{ }^{182}$. Chronic pressure 


\section{Concluding Discussion}

overload, induced by TAC and AAB surgery, however, led to both diastolic and systolic impairment including prolonged relaxation (increase in IVRT), increased left ventricular filling pressure (increases in $\mathrm{E} / \mathrm{E}^{\prime}$, left atrial area and diastolic strain), and contractile dysfunction (decrease in EF). TAC is known to induce a more detrimental phenotype compared to Shunt including systolic dysfunction, increased cardiac fibrosis and higher mortality in mice ${ }^{27}$. Our findings add to this with respect to differences in diastolic assessment. Particularly the presence of cardiac fibrosis is likely to significantly contribute to the progression of diastolic dysfunction following chronic pressure overload.

On a metabolic level, chronic pressure overload caused increased cardiac glucose metabolism including augmented glycolysis, glucose-derived TCA cycle activity, anaplerosis, glutamine synthesis and metabolism respectively including O-GlcNAcylation of proteins (Chapter 4). As discussed in Chapters 1.4 and 4.4, a substrate switch from fatty acids to glucose utilization during pressure overload-induced cardiac hypertrophy has been reported by several studies and is generally accepted ${ }^{122,124,136,137,183}$. However, whether this metabolic switch is a compensatory mechanism to cope with increased haemodynamic stress or in fact is a driver of progression to heart failure is not fully understood and under controversial debate ${ }^{184}$. Several studies have demonstrated beneficial effects of increased glucose utilization during pressure overload. For instance, in mice with an insulin-independent cardiac-specific overexpression of GLUT1, basal glucose uptake and glycolysis were elevated following chronic pressure overload ${ }^{185}$. This was associated with adaptive cardiac remodelling including preserved systolic function, left ventricular dimensions, and higher survival compared to WT littermates. The beneficial cardiac response in transgenic mice was attributed to a favourable energetic profile (PCr/ATP) through increased glucose utilization. Choosing the reverse approach by subjecting cardiomyocytespecific GLUT1-deficient mice to chronic pressure overload, one would have assumed accelerated pathological remodelling and cardiac dysfunction, based on the previous findings. However, despite a shift from glucose to fatty acid utilization in GLUT1-deficient mice, no differences with respect to pathological remodelling compared to WT littermates could be detected following chronic pressure overload ${ }^{186}$. A different way of augmenting glycolysis is through inhibition of FAO (fatty acid oxidation). In this context, heterozygous CPT1 $\beta$ (carnitine palmitoyltransferase $1 \beta$ )-knockout mice were studied following chronic pressure overload ${ }^{187}$. Interestingly, these mice showed exacerbated cardiac hypertrophy, pathological remodelling and premature death compared to WT littermates. This was attributed to increased lipid accumulation 


\section{Concluding Discussion}

and subsequent lipotoxicity due to an inability to uptake long-chain fatty acids into the mitochondria for oxidation. These different findings indicate the complexity between glucose utilization and heart failure development. As outlined before, the metabolic switch may just be a consequence of an inability to use fatty acids; thus, alternative fuels such as glucose are used instead that do not necessarily generate as much ATP in order to sustain myocardial energetics and cardiac function. Pressure overload is associated with a decreased capillary density in the heart leading to a mismatch between oxygen supply and demand ${ }^{73,163}$. With respect to oxygen efficiency, a switch from fatty acid to glucose utilization would be a logical consequence as glucose metabolism is more oxygen efficient than palmitate: for each mole of oxygen consumed, there is a more than $50 \%$ higher production of high energy phosphate bonds from glucose than from palmitate utilization ${ }^{188}$. Each gram of palmitate, on the other hand, produces more high energy bonds than glucose and is therefore more energy efficient, if oxygen is abundant. Taken together, augmenting glucose utilization in the heart during chronic pressure overload does not seem to be detrimental but there is a mixed picture as to whether it is responsible for preventing maladaptive cardiac remodelling or is just a logical consequence due to an insufficient oxygen supply. Glucose metabolism in the heart following two weeks of volume overload remained mainly unchanged with only slight increases in glycolytic activity (Chapter 4). This was also revealed by expression levels of metabolic enzymes. Cardiac function is still well-compensated two weeks after Shunt, thus indicating that glucose utilization might not need to be augmented in order to match a potentially higher demand. As shown in Fig. 5.7, capillary density in the heart is also well-maintained following two weeks of volume overload; hence, as previously mentioned, initial eccentric remodelling and hypertrophy in response to volume overload can be considered as being adaptive and physiological. At this stage, the oxygen supply in the heart is presumably still sufficient so that fatty acids with their favourable energy efficiency remain the ideal substrate for the heart. This is supported by metabolic profiling of inducible, cardiomyocyte-specific c-myc overexpressing mice, who were shown to develop well-compensated left ventricular hypertrophy. Compared to WT littermates, these transgenic animals showed free fatty acid contribution to the TCA cycle to be increased by nearly $50 \%$ with corresponding decreased exogenous glucose contribution in the heart ${ }^{189}$. For future work, it would be of interest to assess volume overloadinduced cardiac remodelling and function after an induced shift from fatty acids to glucose utilization. One potential experiment would be subjecting GLUT1 overexpressing mice to Shunt surgery, which might give new insights into the precise role of the metabolic shift during cardiac 


\section{Concluding Discussion}

remodelling and potentially heart failure development. It would also be interesting to inject labelled palmitate into mice following two weeks of Shunt and TAC respectively with subsequent flux analysis by NMR-spectroscopy. That way it could be assessed whether the increase in glucose metabolism following TAC is associated with decreased fatty acid utilization in vivo. After two weeks of volume overload, fatty acid utilization might still be physiological; thus, there is no need to increase glucose utilization.

From a mechanistic point of view, Nox4-dependent activation of Akt with subsequent protein synthesis and eccentric, adaptive hypertrophy following chronic volume overload has been proposed in this study (Chapter 5). This is in line with previous studies reporting Akt activation to promote adaptive, eccentric hypertrophy during volume overload through increased protein synthesis ${ }^{171,175}$. This finding is similar to the previously reported Akt-dependent increase in physiological hypertrophy following exercise in mice ${ }^{173}$. Thus, at least at relatively early time

points, volume overload and exercise seem to induce similar changes in the heart with respect to remodelling and signalling. Under pathological pressure overload or endothelin-1 stimulation, Akt-deficient mice show less maladaptive hypertrophy. This suggests a beneficial role for Akt activation in cardiac remodelling, both by limiting maladaptive as well as mediating adaptive cardiac hypertrophy under different stress conditions. This role for Akt in cardiac remodelling during different stresses is very similar to what we have proposed for Nox4, as depicted in Fig. 5.14. This suggests an important interaction between both proteins to protect the heart from various stresses via different mechanisms. In line with this hypothesis is a previous study from our group reporting increased levels of Akt-phosphorylation in cardiomyocyte-specific Nox4 overexpressing mice following chronic pressure overload compared to WT littermates ${ }^{73}$. Accordingly, these transgenic animals were protected from cardiac dysfunction and maladaptive remodelling. The exact mechanism how Nox4 might activate Akt has not been further investigated. However, unpublished data from our group demonstrates a Nox4-mediated inhibition of protein phosphatase 2a (PP2A), which subsequently results in increased Aktphosphorylation levels.

Long-term volume overload of 20 weeks, on the other hand, is associated with decreased Akt-, but increased CaMKII (calcium/calmodulin-dependent protein kinase II)-phosphorylation levels, which is known to be an unfavourable signalling pattern ${ }^{27,171}$. This signalling switch after longterm volume overload is associated with increased apoptosis and oxidative stress, potentially triggering the transition to heart failure. Thus, the beneficial and physiological response of the 
heart to volume overload via Nox4-dependent Akt activation seems to be a limited process. Chronic Nox4 activation might in fact have deleterious effects in the heart during volume overload, similar to Akt. Chronic Akt activation can cause maladaptive left ventricular hypertrophy with left ventricular dilation and contractile dysfunction ${ }^{172,190}$. In this regard, longterm effects of Nox4-deficiency during chronic volume overload would be an interesting approach for future work.

Akt activation has also been implicated in the regulation of glucose metabolism. In cancer cells, for instance, activation of Akt leads to increased glucose consumption including augmented glycolysis ${ }^{191}$. In the heart, acute activation of Akt was shown to increase glucose uptake and protect the heart from ischemia-reperfusion injury. Long-term Akt activation in the adult heart, however, contributes to pathological left ventricular hypertrophy, at least partially by reducing mitochondrial oxidative capacity ${ }^{192-194}$. These findings underline the difference between acute versus chronic Akt activation with respect to cardiac remodelling and heart failure respectively. They also seem to be contradictory to our findings. Despite the increased Akt activation following two weeks of volume overload, we could not detect any significant changes in cardiac glucose metabolism, as reported and discussed in Chapter 4. One explanation might be the level of Akt activation. The three studies previously mentioned used transgenic mice with either constitutively active Akt or Akt overexpression, which will result in unphysiological levels of activated Akt. The data presented in our study focused on endogenous Akt-phosphorylation levels, which are only moderately increased by around 30\% through volume overload in WT mice (Fig. 5.10). Additionally, we have used a novel in vivo ${ }^{13} \mathrm{C}$-labelling approach to assess cardiac metabolism, which might also explain the different findings.

From a therapeutic point of view, our study and others demonstrate that enhancing cardiac Nox 4 activation could be an interesting approach during haemodynamic cardiac stress to protect the heart. Prostacyclin has been shown to be a potent Nox4 activator in endothelial cells as prostacyclin treatment induces endothelial cell proliferation, cytoprotection and angiogenesis in a Nox4-dependent manner ${ }^{195}$. It would be interesting to test this hypothesis in a cardiovascular disease model as prostacyclin treatment has been shown to increase both left and right ventricular function in patients suffering from congestive heart failure and pulmonary arterial hypertension respectively ${ }^{196,197}$. Adeno-associated virus (AAV)-mediated gene transfer of Nox4 might be another option for increasing cardiac Nox4 activity in this setting. Many animal studies have used this technique for the transfer of various genes. The most famous one amongst these genes is 


\section{Concluding Discussion}

SERCA-2 $\alpha$ (sarcoplasmic/endoplasmic reticulum calcium ATPase-2 $\alpha$ ), which showed a high potential for heart failure therapy and has been tested in clinical trials ${ }^{198-201}$. However, as outlined before, it is not clear yet whether long-term activation of Nox4 still exerts beneficial effects in the heart during chronic haemodynamic stress. Additionally, many studies report detrimental effects of Nox4 in the development and progression of various diseases including diabetic nephropathy and cancer $^{202-205}$. Thus, general Nox4 activation might also have deleterious side effects. However, Nox4 remains an interesting enzyme for potential heart failure therapy, but substantially more work needs to be done to comprehensively understand its role in cardiovascular diseases.

In conclusion, the work presented in this study highlights the fundamental differences in cardiac physiology, metabolism and distinctive signalling pathways during volume versus pressure overload respectively. Volume overload in the heart is associated with compensated physiological properties, nearly unchanged glucose metabolism and activation of beneficial signalling pathways involving Nox4, Akt and protein synthesis. Pressure overload, on the other hand, causes rapid cardiac dysfunction, induces more complex metabolic alterations including increased glucose and glutamine metabolism, and induces early activation of detrimental signalling such as CaMKII. These findings highlight the importance of haemodynamics, especially the distinction between volume and pressure overload, during cardiac hypertrophy and heart failure development respectively. They also suggest a requirement for differential pharmacological intervention depending on the contribution of volume versus pressure overload in cardiovascular diseases. Surely, this will be challenging as many patients suffer from multiple diseases, such as hypertension and aortic regurgitation, inducing both pressure and volume overload simultaneously. However, a targeted approach like this would allow to treat patients based on their individual haemodynamic profile, which would be of significant benefit for future heart failure therapies. 


\section{References}

1. Vos T, Flaxman AD, Naghavi M, Lozano R, Michaud C, Ezzati M, Shibuya K, Salomon JA, Abdalla S, Aboyans V, et al. Years lived with disability (YLDs) for 1160 sequelae of 289 diseases and injuries 1990-2010: a systematic analysis for the Global Burden of Disease Study 2010. Lancet. 2012;380:2163-2196.

2. Lloyd-Jones D, Adams RJ, Brown TM, Carnethon M, Dai S, De Simone G, Ferguson TB, Ford E, Furie K, Gillespie C, et al. Heart disease and stroke statistics--2010 update: a report from the American Heart Association. Circulation. 2010;121:e46-e215.

3. Zarrinkoub R, Wettermark B, Wandell P, Mejhert M, Szulkin R, Ljunggren G, Kahan T. The epidemiology of heart failure, based on data for 2.1 million inhabitants in Sweden. European journal of heart failure. 2013;15:995-1002.

4. Bui AL, Horwich TB, Fonarow GC. Epidemiology and risk profile of heart failure. Nature reviews. Cardiology. 2011;8:30-41.

5. Heidenreich PA, Albert NM, Allen LA, Bluemke DA, Butler J, Fonarow GC, Ikonomidis JS, Khavjou O, Konstam MA, Maddox TM, et al. Forecasting the impact of heart failure in the United States: a policy statement from the American Heart Association. Circulation. Heart failure. 2013;6:606-619.

6. Levy D, Kenchaiah S, Larson MG, Benjamin EJ, Kupka MJ, Ho KK, Murabito JM, Vasan RS. Long-term trends in the incidence of and survival with heart failure. The New England journal of medicine. 2002;347:1397-1402.

7. Stewart S, MacIntyre K, Hole DJ, Capewell S, McMurray JJ. More 'malignant' than cancer? Five-year survival following a first admission for heart failure. European journal of heart failure. 2001;3:315-322.

8. Chen J, Dharmarajan K, Wang Y, Krumholz HM. National trends in heart failure hospital stay rates, 2001 to 2009. Journal of the American College of Cardiology. 2013;61:10781088.

9. Mozaffarian D, Benjamin EJ, Go AS, Arnett DK, Blaha MJ, Cushman M, Das SR, de Ferranti S, Despres JP, Fullerton HJ, et al. Heart Disease and Stroke Statistics-2016 Update: A Report From the American Heart Association. Circulation. 2016;133:e38-60.

10. Ohlmeier C, Mikolajczyk R, Frick J, Prutz F, Haverkamp W, Garbe E. Incidence, prevalence and 1-year all-cause mortality of heart failure in Germany: a study based on electronic healthcare data of more than six million persons. Clinical research in cardiology : official journal of the German Cardiac Society. 2015;104:688-696. 
11. Yancy CW, Jessup M, Bozkurt B, Butler J, Casey DE, Jr., Drazner MH, Fonarow GC, Geraci SA, Horwich T, Januzzi JL, et al. 2013 ACCF/AHA guideline for the management of heart failure: executive summary: a report of the American College of Cardiology Foundation/American Heart Association Task Force on practice guidelines. Circulation. 2013;128:1810-1852.

12. Senni M, Paulus WJ, Gavazzi A, Fraser AG, Diez J, Solomon SD, Smiseth OA, Guazzi M, Lam CS, Maggioni AP, et al. New strategies for heart failure with preserved ejection fraction: the importance of targeted therapies for heart failure phenotypes. European heart journal. 2014;35:2797-2815.

13. Ziaeian B, Fonarow GC. Epidemiology and aetiology of heart failure. Nature reviews. Cardiology. 2016;13:368-378.

14. Perrino C, Naga Prasad SV, Mao L, Noma T, Yan Z, Kim HS, Smithies O, Rockman HA. Intermittent pressure overload triggers hypertrophy-independent cardiac dysfunction and vascular rarefaction. The Journal of clinical investigation. 2006;116:1547-1560.

15. Pelliccia A, Maron BJ, Spataro A, Proschan MA, Spirito P. The upper limit of physiologic cardiac hypertrophy in highly trained elite athletes. The New England journal of medicine. 1991;324:295-301.

16. Pluim BM, Zwinderman AH, van der Laarse A, van der Wall EE. The athlete's heart. A meta-analysis of cardiac structure and function. Circulation. 2000;101:336-344.

17. Baggish AL, Wang F, Weiner RB, Elinoff JM, Tournoux F, Boland A, Picard MH, Hutter AM, Jr., Wood MJ. Training-specific changes in cardiac structure and function: a prospective and longitudinal assessment of competitive athletes. Journal of applied physiology. 2008;104:1121-1128.

18. Heineke J, Molkentin JD. Regulation of cardiac hypertrophy by intracellular signalling pathways. Nature reviews. Molecular cell biology. 2006;7:589-600.

19. Bostrom P, Mann N, Wu J, Quintero PA, Plovie ER, Panakova D, Gupta RK, Xiao C, MacRae CA, Rosenzweig A, et al. C/EBPbeta controls exercise-induced cardiac growth and protects against pathological cardiac remodeling. Cell. 2010;143:1072-1083.

20. Chung E, Leinwand LA. Pregnancy as a cardiac stress model. Cardiovascular research. 2014;101:561-570.

21. Koitabashi N, Kass DA. Reverse remodeling in heart failure--mechanisms and therapeutic opportunities. Nature reviews. Cardiology. 2012;9:147-157.

22. Cohn JN, Levine TB, Olivari MT, Garberg V, Lura D, Francis GS, Simon AB, Rector T. Plasma norepinephrine as a guide to prognosis in patients with chronic congestive heart failure. The New England journal of medicine. 1984;311:819-823. 
23. Cohn JN, Ferrari R, Sharpe N. Cardiac remodeling--concepts and clinical implications: a consensus paper from an international forum on cardiac remodeling. Behalf of an International Forum on Cardiac Remodeling. Journal of the American College of Cardiology. 2000;35:569-582.

24. Shah AM, Mann DL. In search of new therapeutic targets and strategies for heart failure: recent advances in basic science. Lancet. 2011;378:704-712.

25. Norton JM. Toward consistent definitions for preload and afterload. Advances in physiology education. 2001;25:53-61.

26. Grossman W, Jones D, McLaurin LP. Wall stress and patterns of hypertrophy in the human left ventricle. The Journal of clinical investigation. 1975;56:56-64.

27. Toischer K, Rokita AG, Unsold B, Zhu W, Kararigas G, Sossalla S, Reuter SP, Becker A, Teucher N, Seidler T, et al. Differential cardiac remodeling in preload versus afterload. Circulation. 2010;122:993-1003.

28. Sharpe N, Smith H, Murphy J, Greaves S, Hart H, Gamble G. Early prevention of left ventricular dysfunction after myocardial infarction with angiotensin-converting-enzyme inhibition. Lancet. 1991;337:872-876.

29. Granger CB, McMurray JJ, Yusuf S, Held P, Michelson EL, Olofsson B, Ostergren J, Pfeffer MA, Swedberg K, Investigators C, et al. Effects of candesartan in patients with chronic heart failure and reduced left-ventricular systolic function intolerant to angiotensin-converting-enzyme inhibitors: the CHARM-Alternative trial. Lancet. 2003;362:772-776.

30. Pitt B, Zannad F, Remme WJ, Cody R, Castaigne A, Perez A, Palensky J, Wittes J. The effect of spironolactone on morbidity and mortality in patients with severe heart failure. Randomized Aldactone Evaluation Study Investigators. The New England journal of medicine. 1999;341:709-717.

31. Pitt B, Williams G, Remme W, Martinez F, Lopez-Sendon J, Zannad F, Neaton J, Roniker B, Hurley S, Burns D, et al. The EPHESUS trial: eplerenone in patients with heart failure due to systolic dysfunction complicating acute myocardial infarction. Eplerenone Post-AMI Heart Failure Efficacy and Survival Study. Cardiovascular drugs and therapy / sponsored by the International Society of Cardiovascular Pharmacotherapy. 2001;15:79-87.

32. McMurray JJ, Packer M, Desai AS, Gong J, Lefkowitz MP, Rizkala AR, Rouleau JL, Shi VC, Solomon SD, Swedberg K, et al. Angiotensin-neprilysin inhibition versus enalapril in heart failure. The New England journal of medicine. 2014;371:993-1004.

33. Cleland JG, Daubert JC, Erdmann E, Freemantle N, Gras D, Kappenberger L, Tavazzi L, Cardiac Resynchronization-Heart Failure Study I. The effect of cardiac resynchronization on morbidity and mortality in heart failure. The New England journal of medicine. 2005;352:1539-1549. 
34. Doenst T, Nguyen TD, Abel ED. Cardiac metabolism in heart failure: implications beyond ATP production. Circulation research. 2013;113:709-724.

35. Stanley WC, Recchia FA, Lopaschuk GD. Myocardial substrate metabolism in the normal and failing heart. Physiological reviews. 2005;85:1093-1129.

36. Neubauer S, Horn M, Cramer M, Harre K, Newell JB, Peters W, Pabst T, Ertl G, Hahn D, Ingwall JS, et al. Myocardial phosphocreatine-to-ATP ratio is a predictor of mortality in patients with dilated cardiomyopathy. Circulation. 1997;96:2190-2196.

37. Neglia D, De Caterina A, Marraccini P, Natali A, Ciardetti M, Vecoli C, Gastaldelli A, Ciociaro D, Pellegrini P, Testa R, et al. Impaired myocardial metabolic reserve and substrate selection flexibility during stress in patients with idiopathic dilated cardiomyopathy. American journal of physiology. Heart and circulatory physiology. 2007;293:H3270-3278.

38. Davila-Roman VG, Vedala G, Herrero P, de las Fuentes L, Rogers JG, Kelly DP, Gropler RJ. Altered myocardial fatty acid and glucose metabolism in idiopathic dilated cardiomyopathy. Journal of the American College of Cardiology. 2002;40:271-277.

39. Sack MN, Rader TA, Park S, Bastin J, McCune SA, Kelly DP. Fatty acid oxidation enzyme gene expression is downregulated in the failing heart. Circulation. 1996;94:28372842.

40. Sorokina N, O'Donnell JM, McKinney RD, Pound KM, Woldegiorgis G, LaNoue KF, Ballal K, Taegtmeyer H, Buttrick PM, Lewandowski ED. Recruitment of compensatory pathways to sustain oxidative flux with reduced carnitine palmitoyltransferase I activity characterizes inefficiency in energy metabolism in hypertrophied hearts. Circulation. 2007;115:2033-2041.

41. Abozguia K, Elliott P, McKenna W, Phan TT, Nallur-Shivu G, Ahmed I, Maher AR, Kaur K, Taylor J, Henning A, et al. Metabolic modulator perhexiline corrects energy deficiency and improves exercise capacity in symptomatic hypertrophic cardiomyopathy. Circulation. 2010;122:1562-1569.

42. Tuunanen H, Engblom E, Naum A, Nagren K, Hesse B, Airaksinen KE, Nuutila P, Iozzo P, Ukkonen H, Opie LH, et al. Free fatty acid depletion acutely decreases cardiac work and efficiency in cardiomyopathic heart failure. Circulation. 2006;114:2130-2137.

43. Bersin RM, Wolfe C, Kwasman M, Lau D, Klinski C, Tanaka K, Khorrami P, Henderson $\mathrm{GN}$, de Marco T, Chatterjee $\mathrm{K}$. Improved hemodynamic function and mechanical efficiency in congestive heart failure with sodium dichloroacetate. Journal of the American College of Cardiology. 1994;23:1617-1624.

44. Lewis JF, DaCosta M, Wargowich T, Stacpoole P. Effects of dichloroacetate in patients with congestive heart failure. Clinical cardiology. 1998;21:888-892.

45. Burgoyne JR, Mongue-Din H, Eaton P, Shah AM. Redox signaling in cardiac physiology and pathology. Circulation research. 2012;111:1091-1106. 
46. Denu JM, Tanner KG. Specific and reversible inactivation of protein tyrosine phosphatases by hydrogen peroxide: evidence for a sulfenic acid intermediate and implications for redox regulation. Biochemistry. 1998;37:5633-5642.

47. Sag CM, Santos CX, Shah AM. Redox regulation of cardiac hypertrophy. Journal of molecular and cellular cardiology. 2014;73:103-111.

48. Sanchez G, Pedrozo Z, Domenech RJ, Hidalgo C, Donoso P. Tachycardia increases NADPH oxidase activity and RyR2 S-glutathionylation in ventricular muscle. Journal of molecular and cellular cardiology. 2005;39:982-991.

49. Prysyazhna O, Rudyk O, Eaton P. Single atom substitution in mouse protein kinase G eliminates oxidant sensing to cause hypertension. Nature medicine. 2012;18:286-290.

50. Buggisch M, Ateghang B, Ruhe C, Strobel C, Lange S, Wartenberg M, Sauer H. Stimulation of ES-cell-derived cardiomyogenesis and neonatal cardiac cell proliferation by reactive oxygen species and NADPH oxidase. Journal of cell science. 2007;120:885894.

51. Kameda K, Matsunaga T, Abe N, Hanada H, Ishizaka H, Ono H, Saitoh M, Fukui K, Fukuda I, Osanai T, et al. Correlation of oxidative stress with activity of matrix metalloproteinase in patients with coronary artery disease. Possible role for left ventricular remodelling. European heart journal. 2003;24:2180-2185.

52. Minhas KM, Saraiva RM, Schuleri KH, Lehrke S, Zheng M, Saliaris AP, Berry CE, Barouch LA, Vandegaer KM, Li D, et al. Xanthine oxidoreductase inhibition causes reverse remodeling in rats with dilated cardiomyopathy. Circulation research. 2006;98:271-279.

53. Kaludercic N, Takimoto E, Nagayama T, Feng N, Lai EW, Bedja D, Chen K, Gabrielson KL, Blakely RD, Shih JC, et al. Monoamine oxidase A-mediated enhanced catabolism of norepinephrine contributes to adverse remodeling and pump failure in hearts with pressure overload. Circulation research. 2010;106:193-202.

54. Terentyev D, Gyorke I, Belevych AE, Terentyeva R, Sridhar A, Nishijima Y, de Blanco EC, Khanna S, Sen CK, Cardounel AJ, et al. Redox modification of ryanodine receptors contributes to sarcoplasmic reticulum $\mathrm{Ca} 2+$ leak in chronic heart failure. Circulation research. 2008;103:1466-1472.

55. Sag CM, Wolff HA, Neumann K, Opiela MK, Zhang J, Steuer F, Sowa T, Gupta S, Schirmer M, Hunlich $\mathrm{M}$, et al. Ionizing radiation regulates cardiac $\mathrm{Ca}$ handling via increased ROS and activated CaMKII. Basic research in cardiology. 2013;108:385.

56. Kim YM, Guzik TJ, Zhang YH, Zhang MH, Kattach H, Ratnatunga C, Pillai R, Channon $\mathrm{KM}$, Casadei B. A myocardial Nox2 containing NAD $(\mathrm{P}) \mathrm{H}$ oxidase contributes to oxidative stress in human atrial fibrillation. Circulation research. 2005;97:629-636. 
57. Adam O, Frost G, Custodis F, Sussman MA, Schafers HJ, Bohm M, Laufs U. Role of Rac1 GTPase activation in atrial fibrillation. Journal of the American College of Cardiology. 2007;50:359-367.

58. Palomeque J, Rueda OV, Sapia L, Valverde CA, Salas M, Petroff MV, Mattiazzi A. Angiotensin II-induced oxidative stress resets the $\mathrm{Ca} 2+$ dependence of $\mathrm{Ca} 2+$-calmodulin protein kinase II and promotes a death pathway conserved across different species. Circulation research. 2009;105:1204-1212.

59. Remondino A, Kwon SH, Communal C, Pimentel DR, Sawyer DB, Singh K, Colucci WS. Beta-adrenergic receptor-stimulated apoptosis in cardiac myocytes is mediated by reactive oxygen species/c-Jun NH2-terminal kinase-dependent activation of the mitochondrial pathway. Circulation research. 2003;92:136-138.

60. Graham D, Huynh NN, Hamilton CA, Beattie E, Smith RA, Cocheme HM, Murphy MP, Dominiczak AF. Mitochondria-targeted antioxidant MitoQ10 improves endothelial function and attenuates cardiac hypertrophy. Hypertension. 2009;54:322-328.

61. Adlam VJ, Harrison JC, Porteous CM, James AM, Smith RA, Murphy MP, Sammut IA. Targeting an antioxidant to mitochondria decreases cardiac ischemia-reperfusion injury. FASEB journal : official publication of the Federation of American Societies for Experimental Biology. 2005;19:1088-1095.

62. Lambeth JD. NOX enzymes and the biology of reactive oxygen. Nature reviews. Immunology. 2004;4:181-189.

63. Nabeebaccus A, Zhang M, Shah AM. NADPH oxidases and cardiac remodelling. Heart failure reviews. 2011;16:5-12.

64. Takac I, Schroder K, Zhang L, Lardy B, Anilkumar N, Lambeth JD, Shah AM, Morel F, Brandes RP. The E-loop is involved in hydrogen peroxide formation by the NADPH oxidase Nox4. The Journal of biological chemistry. 2011;286:13304-13313.

65. Zhang M, Perino A, Ghigo A, Hirsch E, Shah AM. NADPH oxidases in heart failure: poachers or gamekeepers? Antioxidants \& redox signaling. 2013;18:1024-1041.

66. Zhang M, Prosser BL, Bamboye MA, Gondim AN, Santos CX, Martin D, Ghigo A, Perino A, Brewer AC, Ward CW, et al. Contractile Function During Angiotensin-II Activation: Increased Nox2 Activity Modulates Cardiac Calcium Handling via Phospholamban Phosphorylation. Journal of the American College of Cardiology. 2015;66:261-272.

67. Johar S, Cave AC, Narayanapanicker A, Grieve DJ, Shah AM. Aldosterone mediates angiotensin II-induced interstitial cardiac fibrosis via a Nox2-containing NADPH oxidase. FASEB journal : official publication of the Federation of American Societies for Experimental Biology. 2006;20:1546-1548.

68. Grieve DJ, Byrne JA, Siva A, Layland J, Johar S, Cave AC, Shah AM. Involvement of the nicotinamide adenosine dinucleotide phosphate oxidase isoform Nox2 in cardiac 
contractile dysfunction occurring in response to pressure overload. Journal of the American College of Cardiology. 2006;47:817-826.

69. Bendall JK, Cave AC, Heymes C, Gall N, Shah AM. Pivotal role of a gp91(phox)containing NADPH oxidase in angiotensin II-induced cardiac hypertrophy in mice. Circulation. 2002;105:293-296.

70. Looi YH, Grieve DJ, Siva A, Walker SJ, Anilkumar N, Cave AC, Marber M, Monaghan MJ, Shah AM. Involvement of Nox2 NADPH oxidase in adverse cardiac remodeling after myocardial infarction. Hypertension. 2008;51:319-325.

71. Krijnen PA, Meischl C, Hack CE, Meijer CJ, Visser CA, Roos D, Niessen HW. Increased Nox2 expression in human cardiomyocytes after acute myocardial infarction. Journal of clinical pathology. 2003;56:194-199.

72. Murray TV, Smyrnias I, Schnelle M, Mistry RK, Zhang M, Beretta M, Martin D, Anilkumar N, de Silva SM, Shah AM, et al. Redox regulation of cardiomyocyte cell cycling via an ERK1/2 and c-Myc-dependent activation of cyclin D2 transcription. Journal of molecular and cellular cardiology. 2015;79:54-68.

73. Zhang M, Brewer AC, Schroder K, Santos CX, Grieve DJ, Wang M, Anilkumar N, Yu B, Dong X, Walker SJ, et al. NADPH oxidase-4 mediates protection against chronic loadinduced stress in mouse hearts by enhancing angiogenesis. Proceedings of the National Academy of Sciences of the United States of America. 2010;107:18121-18126.

74. Moreno MU, Gallego I, Lopez B, Gonzalez A, Fortuno A, San Jose G, Valencia F, Gomez-Doblas JJ, de Teresa E, Shah AM, et al. Decreased Nox4 levels in the myocardium of patients with aortic valve stenosis. Clinical science. 2013;125:291-300.

75. Scheuermann-Freestone M, Freestone NS, Langenickel T, Hohnel K, Dietz R, Willenbrock R. A new model of congestive heart failure in the mouse due to chronic volume overload. European journal of heart failure. 2001;3:535-543.

76. Hu P, Zhang D, Swenson L, Chakrabarti G, Abel ED, Litwin SE. Minimally invasive aortic banding in mice: effects of altered cardiomyocyte insulin signaling during pressure overload. American journal of physiology. Heart and circulatory physiology. 2003;285:H1261-1269.

77. Byrne JA, Grieve DJ, Bendall JK, Li JM, Gove C, Lambeth JD, Cave AC, Shah AM. Contrasting roles of NADPH oxidase isoforms in pressure-overload versus angiotensin IIinduced cardiac hypertrophy. Circulation research. 2003;93:802-805.

78. Schmidt AG, Gerst M, Zhai J, Carr AN, Pater L, Kranias EG, Hoit BD. Evaluation of left ventricular diastolic function from spectral and color M-mode Doppler in genetically altered mice. Journal of the American Society of Echocardiography : official publication of the American Society of Echocardiography. 2002;15:1065-1073.

79. Bhan A, Sirker A, Zhang J, Protti A, Catibog N, Driver W, Botnar R, Monaghan MJ, Shah AM. High-frequency speckle tracking echocardiography in the assessment of left 
ventricular function and remodeling after murine myocardial infarction. American journal of physiology. Heart and circulatory physiology. 2014;306:H1371-1383.

80. Bradford MM. A rapid and sensitive method for the quantitation of microgram quantities of protein utilizing the principle of protein-dye binding. Analytical biochemistry. 1976;72:248-254.

81. Anilkumar N, Weber R, Zhang M, Brewer A, Shah AM. Nox4 and nox2 NADPH oxidases mediate distinct cellular redox signaling responses to agonist stimulation. Arteriosclerosis, thrombosis, and vascular biology. 2008;28:1347-1354.

82. Nkomo VT, Gardin JM, Skelton TN, Gottdiener JS, Scott CG, Enriquez-Sarano M. Burden of valvular heart diseases: a population-based study. Lancet. 2006;368:10051011.

83. Bekeredjian R, Grayburn PA. Valvular heart disease: aortic regurgitation. Circulation. 2005; 112:125-134.

84. Zile MR, Baicu CF, Gaasch WH. Diastolic heart failure--abnormalities in active relaxation and passive stiffness of the left ventricle. The New England journal of medicine. 2004;350:1953-1959.

85. Clark AL, Cleland JG. Causes and treatment of oedema in patients with heart failure. Nature reviews. Cardiology. 2013;10:156-170.

86. Owan TE, Hodge DO, Herges RM, Jacobsen SJ, Roger VL, Redfield MM. Trends in prevalence and outcome of heart failure with preserved ejection fraction. The New England journal of medicine. 2006;355:251-259.

87. Abhayaratna WP, Marwick TH, Smith WT, Becker NG. Characteristics of left ventricular diastolic dysfunction in the community: an echocardiographic survey. Heart. 2006;92:1259-1264.

88. Bhatia RS, Tu JV, Lee DS, Austin PC, Fang J, Haouzi A, Gong Y, Liu PP. Outcome of heart failure with preserved ejection fraction in a population-based study. The New England journal of medicine. 2006;355:260-269.

89. Paulus WJ, Tschope C, Sanderson JE, Rusconi C, Flachskampf FA, Rademakers FE, Marino P, Smiseth OA, De Keulenaer G, Leite-Moreira AF, et al. How to diagnose diastolic heart failure: a consensus statement on the diagnosis of heart failure with normal left ventricular ejection fraction by the Heart Failure and Echocardiography Associations of the European Society of Cardiology. European heart journal. 2007;28:2539-2550.

90. Moller JE, Pellikka PA, Hillis GS, Oh JK. Prognostic importance of diastolic function and filling pressure in patients with acute myocardial infarction. Circulation. 2006;114:438444.

91. Oh JK, Appleton CP, Hatle LK, Nishimura RA, Seward JB, Tajik AJ. The noninvasive assessment of left ventricular diastolic function with two-dimensional and Doppler 
echocardiography. Journal of the American Society of Echocardiography : official publication of the American Society of Echocardiography. 1997;10:246-270.

92. Ommen SR, Nishimura RA, Appleton CP, Miller FA, Oh JK, Redfield MM, Tajik AJ. Clinical utility of Doppler echocardiography and tissue Doppler imaging in the estimation of left ventricular filling pressures: A comparative simultaneous Doppler-catheterization study. Circulation. 2000;102:1788-1794.

93. Tee M, Noble JA, Bluemke DA. Imaging techniques for cardiac strain and deformation: comparison of echocardiography, cardiac magnetic resonance and cardiac computed tomography. Expert review of cardiovascular therapy. 2013;11:221-231.

94. Wang J, Khoury DS, Thohan V, Torre-Amione G, Nagueh SF. Global diastolic strain rate for the assessment of left ventricular relaxation and filling pressures. Circulation. 2007;115:1376-1383.

95. Oh JK, Park SJ, Nagueh SF. Established and novel clinical applications of diastolic function assessment by echocardiography. Circulation. Cardiovascular imaging. 2011;4:444-455.

96. Bauer M, Cheng S, Jain M, Ngoy S, Theodoropoulos C, Trujillo A, Lin FC, Liao R. Echocardiographic speckle-tracking based strain imaging for rapid cardiovascular phenotyping in mice. Circulation research. 2011;108:908-916.

97. Koyama J, Ray-Sequin PA, Falk RH. Longitudinal myocardial function assessed by tissue velocity, strain, and strain rate tissue Doppler echocardiography in patients with AL (primary) cardiac amyloidosis. Circulation. 2003;107:2446-2452.

98. Myreng Y, Smiseth OA. Assessment of left ventricular relaxation by Doppler echocardiography. Comparison of isovolumic relaxation time and transmitral flow velocities with time constant of isovolumic relaxation. Circulation. 1990;81:260-266.

99. Courtois M, Mechem CJ, Barzilai B, Gutierrez F, Ludbrook PA. Delineation of determinants of left ventricular early filling. Saline versus blood infusion. Circulation. 1994;90:2041-2050.

100. Fujimoto N, Borlaug BA, Lewis GD, Hastings JL, Shafer KM, Bhella PS, Carrick-Ranson G, Levine BD. Hemodynamic responses to rapid saline loading: the impact of age, sex, and heart failure. Circulation. 2013;127:55-62.

101. Park TH, Nagueh SF, Khoury DS, Kopelen HA, Akrivakis S, Nasser K, Ren G, Frangogiannis NG. Impact of myocardial structure and function postinfarction on diastolic strain measurements: implications for assessment of myocardial viability. American journal of physiology. Heart and circulatory physiology. 2006;290:H724-731.

102. Oka T, Nishimura H, Ueyama M, Kubota J, Kawamura K. Lisinopril reduces cardiac hypertrophy and mortality in rats with aortocaval fistula. European journal of pharmacology. 1993;234:55-60. 
103. Li Y, Charles PY, Nan C, Pinto JR, Wang Y, Liang J, Wu G, Tian J, Feng HZ, Potter JD, et al. Correcting diastolic dysfunction by $\mathrm{Ca} 2+$ desensitizing troponin in a transgenic mouse model of restrictive cardiomyopathy. Journal of molecular and cellular cardiology. 2010;49:402-411.

104. Li Y, Zhang L, Jean-Charles PY, Nan C, Chen G, Tian J, Jin JP, Gelb IJ, Huang X. Dosedependent diastolic dysfunction and early death in a mouse model with cardiac troponin mutations. Journal of molecular and cellular cardiology. 2013;62:227-236.

105. Moreo A, Ambrosio G, De Chiara B, Pu M, Tran T, Mauri F, Raman SV. Influence of myocardial fibrosis on left ventricular diastolic function: noninvasive assessment by cardiac magnetic resonance and echo. Circulation. Cardiovascular imaging. 2009;2:437443.

106. Nagueh SF, Appleton CP, Gillebert TC, Marino PN, Oh JK, Smiseth OA, Waggoner AD, Flachskampf FA, Pellikka PA, Evangelisa A. Recommendations for the evaluation of left ventricular diastolic function by echocardiography. European journal of echocardiography : the journal of the Working Group on Echocardiography of the European Society of Cardiology. 2009;10:165-193.

107. Erkens R, Kramer CM, Luckstadt W, Panknin C, Krause L, Weidenbach M, Dirzka J, Krenz T, Mergia E, Suvorava T, et al. Left ventricular diastolic dysfunction in Nrf2 knock out mice Is associated with cardiac hypertrophy, decreased expression of SERCA2a, and preserved endothelial function. Free radical biology \& medicine. 2015

108. Fraysse B, Weinberger F, Bardswell SC, Cuello F, Vignier N, Geertz B, Starbatty J, Kramer E, Coirault C, Eschenhagen T, et al. Increased myofilament Ca2+ sensitivity and diastolic dysfunction as early consequences of Mybpc3 mutation in heterozygous knockin mice. Journal of molecular and cellular cardiology. 2012;52:1299-1307.

109. Wilson RM, De Silva DS, Sato K, Izumiya Y, Sam F. Effects of fixed-dose isosorbide dinitrate/hydralazine on diastolic function and exercise capacity in hypertension-induced diastolic heart failure. Hypertension. 2009;54:583-590.

110. Yuan L, Wang T, Liu F, Cohen ED, Patel VV. An evaluation of transmitral and pulmonary venous Doppler indices for assessing murine left ventricular diastolic function. Journal of the American Society of Echocardiography : official publication of the American Society of Echocardiography. 2010;23:887-897.

111. Zhang L, Jaswal JS, Ussher JR, Sankaralingam S, Wagg C, Zaugg M, Lopaschuk GD. Cardiac insulin-resistance and decreased mitochondrial energy production precede the development of systolic heart failure after pressure-overload hypertrophy. Circulation. Heart failure. 2013;6:1039-1048.

112. Szardien S, Nef HM, Voss S, Troidl C, Liebetrau C, Hoffmann J, Rauch M, Mayer K, Kimmich K, Rolf A, et al. Regression of cardiac hypertrophy by granulocyte colonystimulating factor-stimulated interleukin-1beta synthesis. European heart journal. 2012;33:595-605. 
113. Hasegawa H, Little WC, Ohno M, Brucks S, Morimoto A, Cheng HJ, Cheng CP. Diastolic mitral annular velocity during the development of heart failure. Journal of the American College of Cardiology. 2003;41:1590-1597.

114. Tsang TS, Barnes ME, Gersh BJ, Bailey KR, Seward JB. Left atrial volume as a morphophysiologic expression of left ventricular diastolic dysfunction and relation to cardiovascular risk burden. The American journal of cardiology. 2002;90:1284-1289.

115. Abhayaratna WP, Seward JB, Appleton CP, Douglas PS, Oh JK, Tajik AJ, Tsang TS. Left atrial size: physiologic determinants and clinical applications. Journal of the American College of Cardiology. 2006;47:2357-2363.

116. Gonzalez-Quesada C, Cavalera M, Biernacka A, Kong P, Lee DW, Saxena A, Frunza O, Dobaczewski M, Shinde A, Frangogiannis NG. Thrombospondin-1 induction in the diabetic myocardium stabilizes the cardiac matrix in addition to promoting vascular rarefaction through angiopoietin-2 upregulation. Circulation research. 2013;113:13311344.

117. Lorell BH, Apstein CS, Weinberg EO, Cunningham MJ. Diastolic function in left ventricular hypertrophy: clinical and experimental relationships. European heart journal. 1990;11 Suppl G:54-64.

118. Muller-Brunotte R, Kahan T, Malmqvist K, Edner M, Swedish ibesartan left ventricular hypertrophy investigation vs a. Blood pressure and left ventricular geometric pattern determine diastolic function in hypertensive myocardial hypertrophy. Journal of human hypertension. 2003;17:841-849.

119. Miyamoto T, Takeishi Y, Tazawa S, Inoue M, Aoyama T, Takahashi H, Arimoto T, Shishido T, Tomoike H, Kubota I. Fatty acid metabolism assessed by 125I-iodophenyl 9methylpentadecanoic acid (9MPA) and expression of fatty acid utilization enzymes in volume-overloaded hearts. European journal of clinical investigation. 2004;34:176-181.

120. El Alaoui-Talibi Z, Guendouz A, Moravec M, Moravec J. Control of oxidative metabolism in volume-overloaded rat hearts: effect of propionyl-L-carnitine. The American journal of physiology. 1997;272:H1615-1624.

121. Zhabyeyev P, Gandhi M, Mori J, Basu R, Kassiri Z, Clanachan A, Lopaschuk GD, Oudit GY. Pressure-overload-induced heart failure induces a selective reduction in glucose oxidation at physiological afterload. Cardiovascular research. 2013;97:676-685.

122. Nascimben L, Ingwall JS, Lorell BH, Pinz I, Schultz V, Tornheim K, Tian R. Mechanisms for increased glycolysis in the hypertrophied rat heart. Hypertension. 2004;44:662-667.

123. Kolwicz SC, Jr., Olson DP, Marney LC, Garcia-Menendez L, Synovec RE, Tian R. Cardiac-specific deletion of acetyl CoA carboxylase 2 prevents metabolic remodeling during pressure-overload hypertrophy. Circulation research. 2012;111:728-738. 
124. Doenst T, Pytel G, Schrepper A, Amorim P, Farber G, Shingu Y, Mohr FW, Schwarzer M. Decreased rates of substrate oxidation ex vivo predict the onset of heart failure and contractile dysfunction in rats with pressure overload. Cardiovascular research. 2010;86:461-470.

125. Maher EA, Marin-Valencia I, Bachoo RM, Mashimo T, Raisanen J, Hatanpaa KJ, Jindal A, Jeffrey FM, Choi C, Madden C, et al. Metabolism of [U-13 C]glucose in human brain tumors in vivo. NMR in biomedicine. 2012;25:1234-1244.

126. Pardo B, Rodrigues TB, Contreras L, Garzon M, Llorente-Folch I, Kobayashi K, Saheki T, Cerdan S, Satrustegui J. Brain glutamine synthesis requires neuronal-born aspartate as amino donor for glial glutamate formation. Journal of cerebral blood flow and metabolism : official journal of the International Society of Cerebral Blood Flow and Metabolism. 2011;31:90-101.

127. Fan TW, Lane AN, Higashi RM, Yan J. Stable isotope resolved metabolomics of lung cancer in a SCID mouse model. Metabolomics : Official journal of the Metabolomic Society. 2011;7:257-269.

128. Panchal AR, Comte B, Huang H, Kerwin T, Darvish A, des Rosiers C, Brunengraber H, Stanley WC. Partitioning of pyruvate between oxidation and anaplerosis in swine hearts. American journal of physiology. Heart and circulatory physiology. 2000;279:H23902398.

129. Olson AK, Hyyti OM, Cohen GA, Ning XH, Sadilek M, Isern N, Portman MA. Superior cardiac function via anaplerotic pyruvate in the immature swine heart after cardiopulmonary bypass and reperfusion. American journal of physiology. Heart and circulatory physiology. 2008;295:H2315-2320.

130. Lei B, Lionetti V, Young ME, Chandler MP, d'Agostino C, Kang E, Altarejos M, Matsuo $\mathrm{K}$, Hintze TH, Stanley WC, et al. Paradoxical downregulation of the glucose oxidation pathway despite enhanced flux in severe heart failure. Journal of molecular and cellular cardiology. 2004;36:567-576.

131. Damy T, Kirsch M, Khouzami L, Caramelle P, Le Corvoisier P, Roudot-Thoraval F, Dubois-Rande JL, Hittinger L, Pavoine C, Pecker F. Glutathione deficiency in cardiac patients is related to the functional status and structural cardiac abnormalities. PloS one. 2009;4:e4871.

132. Watanabe Y, Watanabe K, Kobayashi T, Saito Y, Fujioka D, Nakamura T, Obata JE, Kawabata K, Mishina H, Kugiyama K. Chronic depletion of glutathione exacerbates ventricular remodelling and dysfunction in the pressure-overloaded heart. Cardiovascular research. 2013;97:282-292.

133. Lunde IG, Aronsen JM, Kvaloy H, Qvigstad E, Sjaastad I, Tonnessen T, Christensen G, Gronning-Wang LM, Carlson CR. Cardiac O-GlcNAc signaling is increased in hypertrophy and heart failure. Physiological genomics. 2012;44:162-172. 
134. Facundo HT, Brainard RE, Watson LJ, Ngoh GA, Hamid T, Prabhu SD, Jones SP. OGlcNAc signaling is essential for NFAT-mediated transcriptional reprogramming during cardiomyocyte hypertrophy. American journal of physiology. Heart and circulatory physiology. 2012;302:H2122-2130.

135. Ngoh GA, Facundo HT, Zafir A, Jones SP. O-GlcNAc signaling in the cardiovascular system. Circulation research. 2010;107:171-185.

136. Allard MF, Schonekess BO, Henning SL, English DR, Lopaschuk GD. Contribution of oxidative metabolism and glycolysis to ATP production in hypertrophied hearts. The American journal of physiology. 1994;267:H742-750.

137. Leong HS, Grist M, Parsons H, Wambolt RB, Lopaschuk GD, Brownsey R, Allard MF. Accelerated rates of glycolysis in the hypertrophied heart: are they a methodological artifact? American journal of physiology. Endocrinology and metabolism. 2002;282:E1039-1045.

138. Mohamed BA, Asif AR, Schnelle M, Qasim M, Khadjeh S, Lbik D, Schott P, Hasenfuss G, Toischer K. Proteomic analysis of short-term preload-induced eccentric cardiac hypertrophy. Journal of translational medicine. 2016;14:149.

139. Kagaya Y, Kanno Y, Takeyama D, Ishide N, Maruyama Y, Takahashi T, Ido T, Takishima T. Effects of long-term pressure overload on regional myocardial glucose and free fatty acid uptake in rats. A quantitative autoradiographic study. Circulation. 1990;81:1353-1361.

140. Tian R, Musi N, D'Agostino J, Hirshman MF, Goodyear LJ. Increased adenosine monophosphate-activated protein kinase activity in rat hearts with pressure-overload hypertrophy. Circulation. 2001;104:1664-1669.

141. Allard MF, Henning SL, Wambolt RB, Granleese SR, English DR, Lopaschuk GD. Glycogen metabolism in the aerobic hypertrophied rat heart. Circulation. 1997;96:676682.

142. Wambolt RB, Henning SL, English DR, Dyachkova Y, Lopaschuk GD, Allard MF. Glucose utilization and glycogen turnover are accelerated in hypertrophied rat hearts during severe low-flow ischemia. Journal of molecular and cellular cardiology. 1999;31:493-502.

143. Wambolt RB, Lopaschuk GD, Brownsey RW, Allard MF. Dichloroacetate improves postischemic function of hypertrophied rat hearts. Journal of the American College of Cardiology. 2000;36:1378-1385.

144. Christe ME, Rodgers RL. Altered glucose and fatty acid oxidation in hearts of the spontaneously hypertensive rat. Journal of molecular and cellular cardiology. 1994;26:1371-1375.

145. Dodd MS, Ball DR, Schroeder MA, Le Page LM, Atherton HJ, Heather LC, Seymour AM, Ashrafian H, Watkins H, Clarke K, et al. In vivo alterations in cardiac metabolism 
and function in the spontaneously hypertensive rat heart. Cardiovascular research. 2012;95:69-76.

146. Hart GW, Slawson C, Ramirez-Correa G, Lagerlof O. Cross talk between OGlcNAcylation and phosphorylation: roles in signaling, transcription, and chronic disease. Annual review of biochemistry. 2011;80:825-858.

147. Slawson C, Copeland RJ, Hart GW. O-GlcNAc signaling: a metabolic link between diabetes and cancer? Trends in biochemical sciences. 2010;35:547-555.

148. Bond MR, Hanover JA. A little sugar goes a long way: the cell biology of O-GlcNAc. The Journal of cell biology. 2015;208:869-880.

149. Pereira RO, Wende AR, Olsen C, Soto J, Rawlings T, Zhu Y, Anderson SM, Abel ED. Inducible overexpression of GLUT1 prevents mitochondrial dysfunction and attenuates structural remodeling in pressure overload but does not prevent left ventricular dysfunction. Journal of the American Heart Association. 2013;2:e00301.

150. Wilkins BJ, Dai YS, Bueno OF, Parsons SA, Xu J, Plank DM, Jones F, Kimball TR, Molkentin JD. Calcineurin/NFAT coupling participates in pathological, but not physiological, cardiac hypertrophy. Circulation research. 2004;94:110-118.

151. Watson LJ, Facundo HT, Ngoh GA, Ameen M, Brainard RE, Lemma KM, Long BW, Prabhu SD, Xuan YT, Jones SP. O-linked beta-N-acetylglucosamine transferase is indispensable in the failing heart. Proceedings of the National Academy of Sciences of the United States of America. 2010;107:17797-17802.

152. Badeer HS. Biological Significance of Cardiac Hypertrophy. The American journal of cardiology. 1964;14:133-138.

153. Linzbach AJ. Heart failure from the point of view of quantitative anatomy. The American journal of cardiology. 1960;5:370-382.

154. Levine ND, Rockoff SD, Braunwald E. An Angiocardiographic Analysis of the Thickness of the Left Ventricular Wall and Cavity in Aortic Stenosis and Other Valvular Lesions. Hemodynamic-Angiographic Correlations in Patients with Obstruction to Left Ventricular Outflow. Circulation. 1963;28:339-345.

155. Ide T, Tsutsui H, Kinugawa S, Suematsu N, Hayashidani S, Ichikawa K, Utsumi H, Machida Y, Egashira K, Takeshita A. Direct evidence for increased hydroxyl radicals originating from superoxide in the failing myocardium. Circulation research. 2000;86:152-157.

156. Cook NR, Albert CM, Gaziano JM, Zaharris E, MacFadyen J, Danielson E, Buring JE, Manson JE. A randomized factorial trial of vitamins $\mathrm{C}$ and $\mathrm{E}$ and beta carotene in the secondary prevention of cardiovascular events in women: results from the Women's Antioxidant Cardiovascular Study. Archives of internal medicine. 2007;167:1610-1618. 
157. Sesso HD, Buring JE, Christen WG, Kurth T, Belanger C, MacFadyen J, Bubes V, Manson JE, Glynn RJ, Gaziano JM. Vitamins E and C in the prevention of cardiovascular disease in men: the Physicians' Health Study II randomized controlled trial. Jama. 2008;300:2123-2133.

158. Yusuf S, Dagenais G, Pogue J, Bosch J, Sleight P. Vitamin E supplementation and cardiovascular events in high-risk patients. The Heart Outcomes Prevention Evaluation Study Investigators. The New England journal of medicine. 2000;342:154-160.

159. Finkel T. Oxidant signals and oxidative stress. Current opinion in cell biology. 2003;15:247-254.

160. Lassegue B, San Martin A, Griendling KK. Biochemistry, physiology, and pathophysiology of NADPH oxidases in the cardiovascular system. Circulation research. 2012;110:1364-1390.

161. Martyn KD, Frederick LM, von Loehneysen K, Dinauer MC, Knaus UG. Functional analysis of Nox4 reveals unique characteristics compared to other NADPH oxidases. Cellular signalling. 2006;18:69-82.

162. Schroder K, Zhang M, Benkhoff S, Mieth A, Pliquett R, Kosowski J, Kruse C, Luedike P, Michaelis UR, Weissmann $\mathrm{N}$, et al. Nox4 is a protective reactive oxygen species generating vascular NADPH oxidase. Circulation research. 2012;110:1217-1225.

163. Smyrnias I, Zhang X, Zhang M, Murray TV, Brandes RP, Schroder K, Brewer AC, Shah AM. Nicotinamide adenine dinucleotide phosphate oxidase-4-dependent upregulation of nuclear factor erythroid-derived 2-like 2 protects the heart during chronic pressure overload. Hypertension. 2015;65:547-553.

164. Brewer AC, Murray TV, Arno M, Zhang M, Anilkumar NP, Mann GE, Shah AM. Nox4 regulates Nrf2 and glutathione redox in cardiomyocytes in vivo. Free radical biology \& medicine. 2011;51:205-215.

165. Kehat I, Davis J, Tiburcy M, Accornero F, Saba-El-Leil MK, Maillet M, York AJ, Lorenz JN, Zimmermann WH, Meloche S, et al. Extracellular signal-regulated kinases 1 and 2 regulate the balance between eccentric and concentric cardiac growth. Circulation research. 2011;108:176-183.

166. Wang X, Proud CG. The mTOR pathway in the control of protein synthesis. Physiology. 2006;21:362-369.

167. Kuroda J, Ago T, Matsushima S, Zhai P, Schneider MD, Sadoshima J. NADPH oxidase 4 (Nox4) is a major source of oxidative stress in the failing heart. Proceedings of the National Academy of Sciences of the United States of America. 2010;107:15565-15570.

168. Zhao QD, Viswanadhapalli S, Williams P, Shi Q, Tan C, Yi X, Bhandari B, Abboud HE. NADPH oxidase 4 induces cardiac fibrosis and hypertrophy through activating Akt/mTOR and NFkappaB signaling pathways. Circulation. 2015;131:643-655. 
169. Shah AM. Parsing the role of NADPH oxidase enzymes and reactive oxygen species in heart failure. Circulation. 2015;131:602-604.

170. Prosser BL, Ward CW, Lederer WJ. X-ROS signaling: rapid mechano-chemo transduction in heart. Science. 2011;333:1440-1445.

171. Mohamed BA, Schnelle M, Khadjeh S, Lbik D, Herwig M, Linke WA, Hasenfuss G, Toischer K. Molecular and structural transition mechanisms in long-term volume overload. European journal of heart failure. 2015

172. Matsui T, Li L, Wu JC, Cook SA, Nagoshi T, Picard MH, Liao R, Rosenzweig A. Phenotypic spectrum caused by transgenic overexpression of activated Akt in the heart. The Journal of biological chemistry. 2002;277:22896-22901.

173. DeBosch B, Treskov I, Lupu TS, Weinheimer C, Kovacs A, Courtois M, Muslin AJ. Akt1 is required for physiological cardiac growth. Circulation. 2006;113:2097-2104.

174. Benard L, Oh JG, Cacheux M, Lee A, Nonnenmacher M, Matasic DS, Kohlbrenner E, Kho C, Pavoine C, Hajjar RJ, et al. Cardiac Stim1 Silencing Impairs Adaptive Hypertrophy and Promotes Heart Failure Through Inactivation of mTORC2/Akt Signaling. Circulation. 2016;133:1458-1471.

175. Ikeda M, Ide T, Fujino T, Matsuo Y, Arai S, Saku K, Kakino T, Oga Y, Nishizaki A, Sunagawa K. The Akt-mTOR axis is a pivotal regulator of eccentric hypertrophy during volume overload. Scientific reports. 2015;5:15881.

176. Xin M, Olson EN, Bassel-Duby R. Mending broken hearts: cardiac development as a basis for adult heart regeneration and repair. Nature reviews. Molecular cell biology. 2013;14:529-541.

177. Cifuentes-Pagano E, Meijles DN, Pagano PJ. The quest for selective nox inhibitors and therapeutics: challenges, triumphs and pitfalls. Antioxidants \& redox signaling. 2014;20:2741-2754.

178. Cifuentes-Pagano ME, Meijles DN, Pagano PJ. Nox Inhibitors \& Therapies: Rational Design of Peptidic and Small Molecule Inhibitors. Current pharmaceutical design. 2015;21:6023-6035.

179. Yamamoto K, Protack CD, Tsuneki M, Hall MR, Wong DJ, Lu DY, Assi R, Williams WT, Sadaghianloo N, Bai $\mathrm{H}$, et al. The mouse aortocaval fistula recapitulates human arteriovenous fistula maturation. American journal of physiology. Heart and circulatory physiology. 2013;305:H1718-1725.

180. Moller JE, Sondergaard E, Poulsen SH, Egstrup K. Pseudonormal and restrictive filling patterns predict left ventricular dilation and cardiac death after a first myocardial infarction: a serial color M-mode Doppler echocardiographic study. Journal of the American College of Cardiology. 2000;36:1841-1846. 
181. Burlew BS, Weber KT. Cardiac fibrosis as a cause of diastolic dysfunction. Herz. 2002;27:92-98.

182. Kass DA, Bronzwaer JG, Paulus WJ. What mechanisms underlie diastolic dysfunction in heart failure? Circulation research. 2004;94:1533-1542.

183. Akki A, Smith K, Seymour AM. Compensated cardiac hypertrophy is characterised by a decline in palmitate oxidation. Molecular and cellular biochemistry. 2008;311:215-224.

184. van Bilsen M, van Nieuwenhoven FA, van der Vusse GJ. Metabolic remodelling of the failing heart: beneficial or detrimental? Cardiovascular research. 2009;81:420-428.

185. Liao R, Jain M, Cui L, D'Agostino J, Aiello F, Luptak I, Ngoy S, Mortensen RM, Tian R. Cardiac-specific overexpression of GLUT1 prevents the development of heart failure attributable to pressure overload in mice. Circulation. 2002;106:2125-2131.

186. Pereira RO, Wende AR, Olsen C, Soto J, Rawlings T, Zhu Y, Riehle C, Abel ED. GLUT1 deficiency in cardiomyocytes does not accelerate the transition from compensated hypertrophy to heart failure. Journal of molecular and cellular cardiology. 2014;72:95103.

187. He L, Kim T, Long Q, Liu J, Wang P, Zhou Y, Ding Y, Prasain J, Wood PA, Yang Q. Carnitine palmitoyltransferase-1b deficiency aggravates pressure overload-induced cardiac hypertrophy caused by lipotoxicity. Circulation. 2012;126:1705-1716.

188. Kessler G, Friedman J. Metabolism of fatty acids and glucose. Circulation. 1998;98:1351.

189. Olson AK, Ledee D, Iwamoto K, Kajimoto M, O'Kelly Priddy C, Isern N, Portman MA. C-Myc induced compensated cardiac hypertrophy increases free fatty acid utilization for the citric acid cycle. Journal of molecular and cellular cardiology. 2013;55:156-164.

190. Hua Y, Zhang Y, Ceylan-Isik AF, Wold LE, Nunn JM, Ren J. Chronic Akt activation accentuates aging-induced cardiac hypertrophy and myocardial contractile dysfunction: role of autophagy. Basic research in cardiology. 2011;106:1173-1191.

191. Elstrom RL, Bauer DE, Buzzai M, Karnauskas R, Harris MH, Plas DR, Zhuang H, Cinalli RM, Alavi A, Rudin CM, et al. Akt stimulates aerobic glycolysis in cancer cells. Cancer research. 2004;64:3892-3899.

192. Matsui T, Nagoshi T, Hong EG, Luptak I, Hartil K, Li L, Gorovits N, Charron MJ, Kim JK, Tian R, et al. Effects of chronic Akt activation on glucose uptake in the heart. American journal of physiology. Endocrinology and metabolism. 2006;290:E789-797.

193. Matsui T, Tao J, del Monte F, Lee KH, Li L, Picard M, Force TL, Franke TF, Hajjar RJ, Rosenzweig A. Akt activation preserves cardiac function and prevents injury after transient cardiac ischemia in vivo. Circulation. 2001;104:330-335.

194. Wende AR, O'Neill BT, Bugger H, Riehle C, Tuinei J, Buchanan J, Tsushima K, Wang L, Caro P, Guo A, et al. Enhanced cardiac Akt/protein kinase B signaling contributes to 
pathological cardiac hypertrophy in part by impairing mitochondrial function via transcriptional repression of mitochondrion-targeted nuclear genes. Molecular and cellular biology. 2015;35:831-846.

195. Peshavariya HM, Liu GS, Chang CW, Jiang F, Chan EC, Dusting GJ. Prostacyclin signaling boosts NADPH oxidase 4 in the endothelium promoting cytoprotection and angiogenesis. Antioxidants \& redox signaling. 2014;20:2710-2725.

196. Yui Y, Nakajima H, Kawai C, Murakami T. Prostacyclin therapy in patients with congestive heart failure. The American journal of cardiology. 1982;50:320-324.

197. Brittain EL, Pugh ME, Wheeler LA, Robbins IM, Loyd JE, Newman JH, Austin ED, Hemnes AR. Prostanoids but not oral therapies improve right ventricular function in pulmonary arterial hypertension. JACC. Heart failure. 2013;1:300-307.

198. del Monte F, Williams E, Lebeche D, Schmidt U, Rosenzweig A, Gwathmey JK, Lewandowski ED, Hajjar RJ. Improvement in survival and cardiac metabolism after gene transfer of sarcoplasmic reticulum $\mathrm{Ca}(2+)$-ATPase in a rat model of heart failure. Circulation. 2001;104:1424-1429.

199. Miyamoto MI, del Monte F, Schmidt U, DiSalvo TS, Kang ZB, Matsui T, Guerrero JL, Gwathmey JK, Rosenzweig A, Hajjar RJ. Adenoviral gene transfer of SERCA2a improves left-ventricular function in aortic-banded rats in transition to heart failure. Proceedings of the National Academy of Sciences of the United States of America. 2000;97:793-798.

200. Hajjar RJ, Zsebo K, Deckelbaum L, Thompson C, Rudy J, Yaroshinsky A, Ly H, Kawase Y, Wagner K, Borow K, et al. Design of a phase 1/2 trial of intracoronary administration of AAV1/SERCA2a in patients with heart failure. Journal of cardiac failure. 2008; $14: 355-367$.

201. Jaski BE, Jessup ML, Mancini DM, Cappola TP, Pauly DF, Greenberg B, Borow K, Dittrich H, Zsebo KM, Hajjar RJ, et al. Calcium upregulation by percutaneous administration of gene therapy in cardiac disease (CUPID Trial), a first-in-human phase 1/2 clinical trial. Journal of cardiac failure. 2009;15:171-181.

202. Gorin Y, Block K. Nox4 and diabetic nephropathy: with a friend like this, who needs enemies? Free radical biology \& medicine. 2013;61:130-142.

203. Gorin Y, Block K, Hernandez J, Bhandari B, Wagner B, Barnes JL, Abboud HE. Nox4 $\mathrm{NAD}(\mathrm{P}) \mathrm{H}$ oxidase mediates hypertrophy and fibronectin expression in the diabetic kidney. The Journal of biological chemistry. 2005;280:39616-39626.

204. Graham KA, Kulawiec M, Owens KM, Li X, Desouki MM, Chandra D, Singh KK. NADPH oxidase 4 is an oncoprotein localized to mitochondria. Cancer biology \& therapy. 2010;10:223-231. 
205. Zhang C, Lan T, Hou J, Li J, Fang R, Yang Z, Zhang M, Liu J, Liu B. NOX4 promotes non-small cell lung cancer cell proliferation and metastasis through positive feedback regulation of PI3K/Akt signaling. Oncotarget. 2014;5:4392-4405. 


\section{Curriculum vitae}

\section{Personal information:}

name:

date of birth:

place of birth:

\section{Secondary school:}

1996 - 2005:

2002 - 2003:

\section{Medical education:}

April 2006 - May 2013:

April 2008:

May 2013:

\section{Scientific education:}

April 2008 - August 2012: in the regulation of cell adhesion in tumor cells) in the

Department of Cardiovascular Physiology at the University of Goettingen under supervision of Prof. D.M. Katschinski (grade of the dissertation: "magna cum laude", grade of the disputation: "summa cum laude")

February - August 2011: $\quad$ Scientific internship in the laboratory of Prof. Randall S. Johnson in the Department of Biological Sciences at the University of California, San Diego, USA

funded by the University of Goettingen with the Gö4med-scholarship June 2013 - ongoing: $\quad$ PhD student in the International Research Training Group 1816 at the University of Goettingen/King's College London, funded by the German Research Foundation. Thesis title: Physiology, metabolism and redox mechanisms in chronic cardiac volume overload, supervised by Prof. A. Shah and Prof. G. Hasenfuß 


\section{$\underline{\text { Presentations }}$}

$10 / 2013$

09/2015

04/2016

06/2016

$06 / 2016$
Talk at the IRTG1816 Retreat in Uslar, Germany

Talk at the IRTG1816/IRTG1902 Network Meeting in Werningerode, Germany

Poster presentation at the ISHR (International Society for Heart Research) World Congress in Buenos Aires, Argentina

Talk at the Gordon Research Seminar (GRS) on NADPH Oxidases in Waterville Valley, USA

Poster presentation at the Gordon Research Conference (GRC) on NADPH Oxidases in Waterville Valley, USA 


\section{Publications:}

Vogel S, Wottawa M, Farhat K, Zieseniss A, Schnelle M, Le-Huu S, von Ahlen M, Malz C, Camenisch G, Katschinski DM (2010): Prolyl hydroxylase domain (PHD) 2 affects cell migration and F-actin formation via RhoA/rho-associated kinase-dependent cofilin phosphorylation. J Biol Chem 285: 33756-63.

Branco-Price C, Zhang N, Schnelle M, Evans C, Katschinski DM, Liao D, Ellies L, Johnson RS (2012): Endothelial cell HIF-1 $\alpha$ and HIF-2 $\alpha$ differentially regulate metastatic success. Cancer Cell 21: 52-65.

Wottawa M, Leisering P, von Ahlen M, Schnelle M, Vogel S, Malz C, Bordoli MR, Camenisch G, Hesse A, Napp J et al. (2013): Knockdown of Prolyl-4-hydroxylase domain 2 inhibits tumor growth of human breast cancer MDA-MB-231 cells by affecting TGF- $\beta 1$ processing. Int J Cancer 132: 2787-98.

Murray TV, Smyrnias I, Schnelle M, Mistry RK, Zhang M, Beretta M, Martin D, Anilkumar N, de Silva SM, Shah AM et al. (2015): Redox regulation of cardiomyocyte cell cycling via an ERK1/2 and c-Myc-dependent activation of cyclin D2 transcription. J Mol Cell Cardiol 79: 5468.

Mohamed BA, Schnelle M, Khadjeh S, Lbik D, Herwig M, Linke WA, Hasenfuss G, Toischer K (2016): Molecular and structural transition mechanisms in long-term volume overload. Eur J Heart Fail 4: 362-71.

Mohamed BA, Asif AR, Schnelle M, Qasim M, Khadjeh S, Lbik D, Schott P, Hasenfuss G, Toischer K (2016): Proteomic analysis of short-term preload-induced eccentric cardiac hypertrophy. J Transl Med 14:149.

Sag CM*, Schnelle M*, Zhang J*, Murdoch C, Protti A, Santos CX, Sawyer G, Mongue-Din H, Richards D, Brewer AC, Prysyazhna O, Maier LS, Eaton PJ, Shah AM. Distinct regulatory effects of myeloid cell and endothelial cell Nox2 on blood pressure (submitted to Circulation in June 2016, currently under revision)

* equal contribution 